\title{
Regioselective Alkyne Cyclotrimerization with an In Situ-Generated [Fe(II)H(salen)]·Bpin Catalyst
}

\author{
Cei B. Provis-Evans, ${ }^{\dagger}$ Samantha Lau, ${ }^{+}$Vera Krewald, ${ }^{*, \ddagger}$ and Ruth L. Webster ${ }^{*,+}$ \\ ${ }^{\dagger}$ Department of Chemistry, University of Bath, Claverton Down, Bath BA2 7AY, \\ United Kingdom \\ ${ }^{\ddagger}$ Department of Chemistry, Theoretical Chemistry, TU Darmstadt, Alarich-Weiss-St. 4, 64287 \\ Darmstadt, Germany \\ $\underline{\text { Table of Contents }}$
}













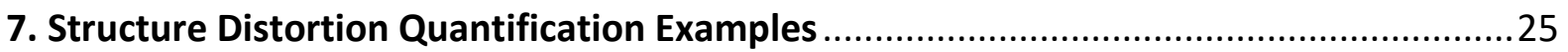

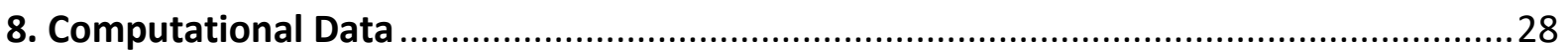





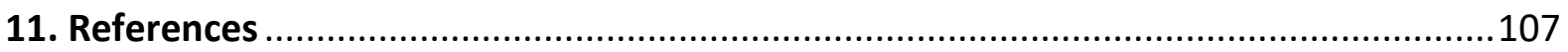




\title{
1. General considerations
}

Reagents were purchased from Sigma Aldrich, Alfa Aesar or Fisher and if required were distilled before use. Laboratory grade acetonitrile was purchased from Sigma-Aldrich and was dried over calcium hydride before use, in addition to being used without further purification to quench the reactions. NMR data was collected at 300 and $500 \mathrm{MHz}$ on Bruker or Agilent instruments in $\mathrm{CDCl}_{3}$ at $298 \mathrm{~K}$ and referenced to residual protic solvent. Heated and anhydrous reactions were undertaken in Teflon-sealed J-Young reaction tubes and Schlenk tubes. Manipulations were carried out under an inert atmosphere using standard Schlenk and glovebox techniques, unless otherwise stated. All mass spectra were obtained with Atmospheric Pressure Solids Analysis Probe (ASAP), or Nanospray lonisation (NSI) in positive mode.

\section{General method for cyclotrimerization of alkynes}

Manipulations were carried out under an argon atmosphere in an M-Braun glove box. 1 (1.7 $\mathrm{mg}, 0.0025 \mathrm{mmol}, 1 \mathrm{~mol} \%)$, Alkyne $(0.25 \mathrm{mmol})$ and pinacolborane $(14.5 \mu \mathrm{l}, 0.10 \mathrm{mmol}, 40$ mol\%) were added to a J. Young valve NMR tube. $600 \mu \mathrm{l}$ of dry MeCN was then added under $\mathrm{N}_{2}$. The sealed reaction was maintained at the required temperature for the time specified for each substrate. The product was isolated by exposing the reaction mixture to air, and adding $1 \mathrm{ml}$ of bench MeCN to quench the remaining pinacolborane. This mixture was then passed through a plug of alumina to remove residual iron and boronic acid. If solid remained in the NMR tube, this was re-dissolved in a minimum of $\mathrm{CHCl}_{3}$ and also passed through the alumina plug. The solvent was removed under a flow of $\mathrm{N}_{2}$, and the product dried in vacuo. $\mathrm{CDCl}_{3}$ was used as the NMR solvent.

\section{Other reactions}

Blank experiments were carried out on phenylacetylene without 1 and with pinacolborane, and with 1 without pinacolborane otherwise utilising the general method above. No reaction was observed in either of these experiments.

Testing of $\mathrm{FeCl}_{2}$ and $\mathrm{FeCl}_{3}$ as catalysts at 1 mol\% under the conditions specified in the general method yielded no product after 1 hour at room temperature using the work-up specified above.

Radical trap experiment conducted using PA as per general method, with the addition of 0.25 $\mathrm{mmol}$ of cyclopropane methyl chloride. No inhibition or alkene formation observed.

\section{Synthesis of Iron Complexes}

\author{
Synthesis of [Fe(salen) $]_{2}-\mu-o x o(1)^{[1]}$
}




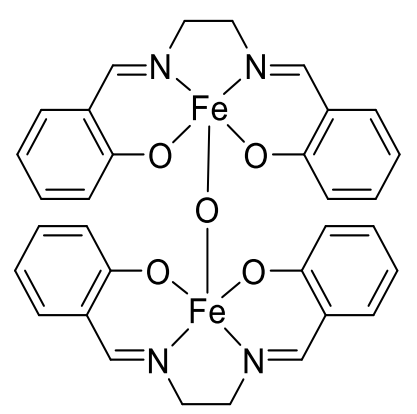

\section{Ligand synthesis:}<smiles>O=Cc1ccccc1O</smiles><smiles>NCCN</smiles><smiles></smiles><smiles>Oc1ccccc1/C=N/CC/N=C/c1ccccc1O</smiles>

A mixture of salicylaldehyde $(2.442 \mathrm{~g}, 20.0 \mathrm{mmol}, 2 \mathrm{eq})$ and ethylenediamine $(0.602 \mathrm{~g}, 10.0$ mmol, 1 eq) in EtOH ( $45 \mathrm{ml}$ ) was heated at reflux for $2 \mathrm{~h}$. The solution was cooled to $-20^{\circ} \mathrm{C}$ and filtered, and the resultant yellow solid was washed with cold EtOH. The product was then dried under vacuum to yield $2.470 \mathrm{~g}$ of a yellow crystalline solid ( $92 \%$ yield).

${ }^{1} \mathrm{H}$ NMR $\left(\mathrm{CDCl}_{3}, 500 \mathrm{MHz}\right): \delta 13.19(\mathrm{~s}, 2 \mathrm{H} \mathrm{Ar}-\mathrm{OH}), 8.36(\mathrm{~s}, 2 \mathrm{H}, \mathrm{N}=\mathrm{CH}), 7.31-7.27(\mathrm{~m}, 2 \mathrm{H}, \mathrm{Ar}-\mathrm{H})$, 7.24-7.22 (m, 2H, Ar-H), 6.95-6.93 (m, 2H, Ar-H), 6.87-6.84 (m, 2H, Ar-H), 3.95 (s, 4H,N-CH2$\mathrm{CH} 2-\mathrm{N})$. Concordant with literature. ${ }^{[2]}$

\section{Complex synthesis:}


$\mathrm{Fe}(\mathrm{OAc})_{2}(0.943 \mathrm{~g}, 5.42 \mathrm{mmol}, 1$ equiv.) was weighed into a flask and dissolved in $\mathrm{EtOH}$ (45 $\mathrm{ml}$ ) leaving a brown solution. To this, a solution of $\mathrm{N}, \mathrm{N}^{\prime}$-Bis(salicylidene)ethylenediamine ( $1.744 \mathrm{~g}, 6.50 \mathrm{mmol}, 1.2$ equiv.) in $\mathrm{EtOH}(90 \mathrm{ml})$ was added to form a red solution. The mixture was stirred at $80^{\circ} \mathrm{C}$ for $2 \mathrm{~h}$. The flask was allowed to cool to RT before filtering off the solid and washing it with EtOH. The solid obtained was dried under vacuum. $1.8762 \mathrm{~g}$ of a dark $\mathrm{red} / \mathrm{brown}$ solid was obtained ( $52 \%$ yield).

IR (solid) v 3049, 1610, 1597, $851 \mathrm{~cm}^{-1}$; m.p. (decomp.) $296^{\circ} \mathrm{C}$.

${ }^{1} \mathrm{H}$ NMR (CDCl, $500 \mathrm{MHz}$ ): $\delta$ (all br.) 76.49, 47.28, 25.02, 20.51, 12.85, 10.13, 1.95, -66.18, 81.25 
Crystallised from MeCN by slow evaporation:

Dark red/brown solid (143 mg, 72 \%). IR (solid) v 3049, 1610, 1597, $851 \mathrm{~cm}^{-1}$; m.p. (decomp.) $296{ }^{\circ} \mathrm{C}^{[1]}$

Crystal structure obtained, compound data is comparable to literature. ${ }^{[2-8]}$

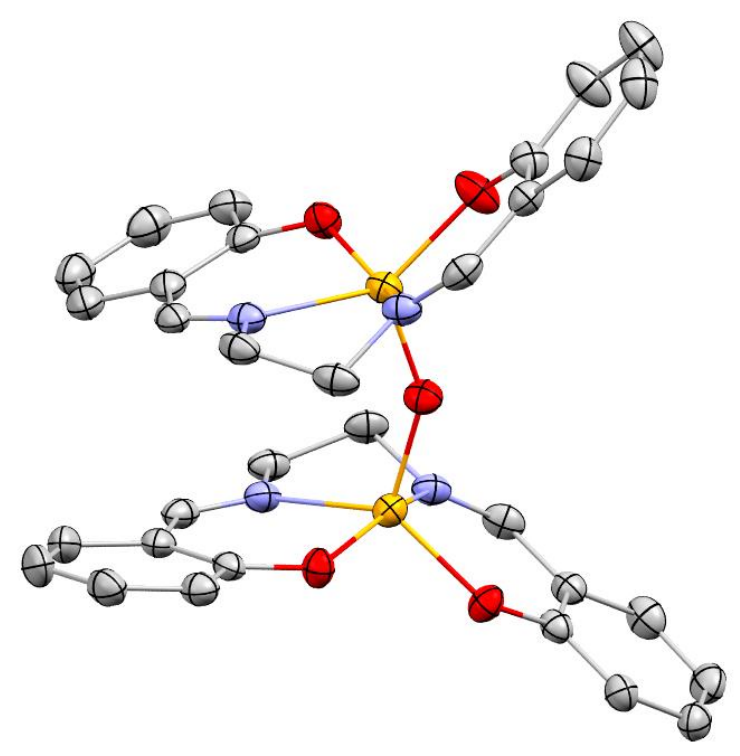

Table S1: Crystal data and structure refinement parameters for $1 .^{[1]}$

\begin{tabular}{|l|l|}
\hline ID Code & K14rlw1 \\
\hline Empirical formula & C32 H28 Fe2 N4 O5 \\
\hline Formula weight & 660.28 \\
\hline Temperature & $150(2) \mathrm{K}$ \\
\hline Wavelength & 0.71073 \\
\hline Crystal system & Triclinic \\
\hline Space group & $\mathrm{P}-1$ \\
\hline Unit cell dimensions & $\mathrm{a}=10.7340(3) \AA ̊ \AA$ alpha $=67.142(1)^{\circ}$ \\
\hline & $\mathrm{b}=10.8150(2) \AA ̊ \AA$ beta $=85.858(1)^{\circ}$ \\
\hline & $\mathrm{C}=13.7380(4) \AA$ gamma $=73.156(2)^{\circ}$ \\
\hline Volume & $1405.06(6) \AA^{3}$ \\
\hline Z & 2 \\
\hline Density (calculated) & $1.561 \mathrm{Mg} / \mathrm{m}^{3}$ \\
\hline Absorption coefficient & $1.083 \mathrm{~mm}^{-1}$ \\
\hline F(000) & 680 \\
\hline Crystal size & $0.15 \times 0.1 \times 0.07 \mathrm{~mm}$ \\
\hline Theta range for data collection & $3.71 \mathrm{to} 24.99^{\circ}$ \\
\hline Index ranges & $-12 \leq \mathrm{h} \leq 12 ;-12 \leq \mathrm{k} \leq 12 ;-16 \leq \mathrm{I} \leq 16 ;$ \\
\hline
\end{tabular}




\begin{tabular}{|l|l|}
\hline Reflections collected & 20793 \\
\hline Independent reflections & $4911[\mathrm{R}($ int $)=0.0669]$ \\
\hline Reflections observed (>2 sigma) & 3673 \\
\hline Data completeness & 0.992 \\
\hline Absorption correction & Semi-empirical from equivalents \\
\hline Max. and min. Transmission & 0.989 and 0.899 \\
\hline Refinement method & Full-matrix least-squares on $\mathrm{F}^{2}$ \\
\hline Data / restraints / parameters & $4911 / 0 / 388$ \\
\hline Goodness-of-fit on $\mathrm{F}^{2}$ & 1.035 \\
\hline Final R indices [I > 2 sigma(I)] & $\mathrm{R} 1=0.0404 \quad W R 2=0.0837$ \\
\hline R indices (all data) & $\mathrm{R} 1=0.0672 \quad W R 2=0.0933$ \\
\hline Largest diff. peak and hole & 0.319 and $-0.365 \mathrm{e}^{3}$ \\
\hline
\end{tabular}

Table S2: Elemental Analysis of $\mathbf{1}^{[1]}$

\begin{tabular}{|l|l|l|l|}
\hline Element & Expected (\%) & Found 1 (\%) & Found 2 (\%) \\
\hline Carbon & 59.66 & 59.47 & 59.45 \\
\hline Hydrogen & 4.38 & 4.30 & 4.26 \\
\hline Nitrogen & 8.70 & 8.61 & 8.55 \\
\hline
\end{tabular}

Synthesis of [Fe(salen)] (4) ${ }^{[2]}$ No. 1<smiles></smiles>

Ligand synthesis as for 1

\section{Complex synthesis:}




A potentiostat was connected to a voltage amplifier and used in direct current mode with a target voltage of $10 \mathrm{~V}$. The working electrode was a piece of high purity iron foil (Goodfellow, $0.075 \mathrm{~mm}$ thick, $99.5 \%$ pure) while the counter-electrode was a piece of twisted steel wire (taken from the handle of a lab brush). The electrodes were placed in a dry 3 neck vessel under a flow of $\mathrm{N}_{2}$, and $\mathrm{N}, \mathrm{N}^{\prime}$-Bis(salicylidene)ethylenediamine (268 mg, $1.0 \mathrm{mmol}, 1$ equiv.) and $\mathrm{Bu}_{4} \mathrm{~N}_{\mathrm{BF}} \mathrm{BF}_{4}(10 \mathrm{mg}, 0.03 \mathrm{mmol}, 0.03$ equiv.) were added, and dissolved in dry MeCN ( $25 \mathrm{ml})$. $\mathrm{N}_{2}$ was bubbled through this solution with stirring to ensure that it was fully degassed, and the potentiostat was turned on, giving an initial PD of $\sim 5 \mathrm{~V}$ and a current of $\sim 4 \mathrm{~mA}$. This was allowed to react for two days with stirring, by which time the current had dropped to $\sim 1 \mathrm{~mA}$ and a large amount of dark violet precipitate was observable. The dark purple MeCN supernatant was removed by filter cannula, and the precipitate was washed with two aliquots dry $\mathrm{MeCN}(10 \mathrm{ml})$ and two aliquots of dry $\mathrm{Et}_{2} \mathrm{O}(10 \mathrm{ml})$ under $\mathrm{Ar}$, before drying under vacuum to yield $145.1 \mathrm{mg}$ of a pale violet powder ( $45 \%$ yield). Attempts were made to crystallise the complex for X-ray crystallography without success.

${ }^{1} \mathrm{H}$ NMR (CD ${ }_{3} \mathrm{CN}, 500 \mathrm{MHz}$ ): $\delta$ (all br.) 56.64, 48.69, 12.89, 10.17, 7.62, 7.41, 6.05, 5.48, 3.10 $(\mathrm{t}), 1.63,1.38(\mathrm{q}), 1.00(\mathrm{t}),-13.02,-20.63$

\section{Synthesis of [Fe(salen)] (4) No. 2}<smiles></smiles>

\section{Ligand synthesis as for 1}

\section{Complex synthesis:}


A solution of $\mathrm{N}, \mathrm{N}^{\prime}$-Bis(salicylidene)ethylenediamine ( $268 \mathrm{mg}, 1.0 \mathrm{mmol}, 1$ equiv.) in dry THF $(10 \mathrm{ml})$ was prepared in a Schlenk tube under $\mathrm{N}_{2}$. To this $1.382 \mathrm{ml}$ (2.1 equiv.) of $\mathrm{n}$-BuLi (1.52 $\mathrm{M}$ solution in THF) was added at $-78^{\circ} \mathrm{C}$, and the solution allowed to warm to $0{ }^{\circ} \mathrm{C}$ with stirring. This solution was then added slowly to a solution of $\mathrm{FeCl}_{2}$. THF $(235 \mathrm{mg}, 1.0 \mathrm{mmol}, 1 \mathrm{eq})$ in THF $(20 \mathrm{ml})$ in another Schlenk tube via a cannula. The mixture was allowed to warm to room temperature, and stirred for $2 \mathrm{~h}$ yielding a purple solution. It proved impossible to remove the by-product LiCl from this the material, so the solvent was removed under vacuum to yield 
a dark violet air sensitive solid with together with $\mathrm{LiCl}$. Attempts were made to crystallise the complex for X-ray crystallography without success.

${ }^{1} \mathrm{H}$ NMR (CD 3 CN, $500 \mathrm{MHz}$ ): $\delta$ (all br.) 33.24, 28.64, 23.48, 18.91, 8.09, 3.58, 1.79, 0.90, -2.69, $-12.20$

\section{Synthesis of [Fe(PhSalen) $]_{2}-\mu$-oxo (6)}



Ligand synthesis:<smiles>O=Cc1ccccc1O</smiles><smiles>Nc1ccccc1N</smiles>


A mixture of salicylaldehyde $(2.442 \mathrm{~g}, 20 \mathrm{mmol}, 2$ equiv. $)$ and 1,2-phenyldiamine $(1.081 \mathrm{~g}, 10$ mmol, 1 equiv.) in dry EtOH ( $45 \mathrm{ml}$ ) was heated at reflux with stirring for $2 \mathrm{~h}$. The solution was cooled to $-20^{\circ} \mathrm{C}$ and filtered, and the resultant yellow solid was washed with cold EtOH. The product was then dried in vacuo. $2.5215 \mathrm{~g}$ of orange crystalline solid obtained ( $80 \%$ yield)

${ }^{1} \mathrm{H}$ NMR $\left(\mathrm{CDCl}_{3}, 500 \mathrm{MHz}\right): \delta 13.01$ (br s, 2H, Ar-OH), 8.65 (s, 2H, N=CH), 7.41-7.35 (m, 6H, ArH), 7.27-7.25 (m, 4H, Ar-H), 7.07 (d, J=8.24 Hz, 2H, Ar-H), 6.94 (td, J= 7.49, $1.11 \mathrm{~Hz}, 2 \mathrm{H}$, Ar$H) ;{ }^{13} \mathrm{C} \mathrm{NMR}\left(\mathrm{CDCl}_{3}, 126 \mathrm{MHz}\right): \delta$ 163.7, 161.4, 142.52, 133.4, 132.3, 127.7, 119.8, 199.2, 119.0, 117.6; concordant with literature. ${ }^{[2]}$ 


\section{Complex synthesis:}

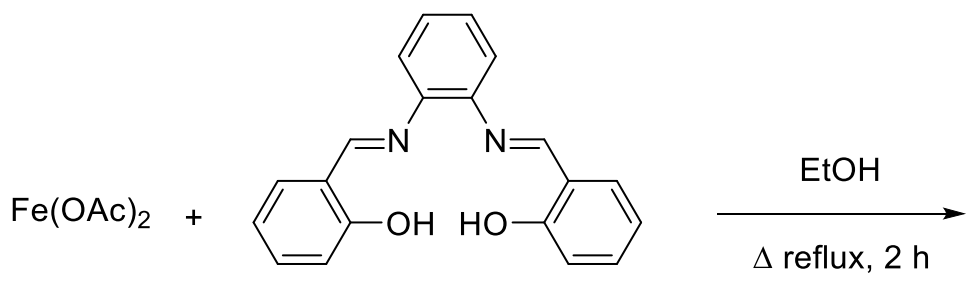



$\mathrm{Fe}(\mathrm{OAc})_{2}$ (435 mg, $2.50 \mathrm{mmol}, 1$ equiv.) was weighed into a flask and dissolved in EtOH (40 $\mathrm{ml}$ ) leaving a brown suspension. To this, a solution of N,N'-Bis(salicylidene)-1,2phenylenediamine ( $949 \mathrm{mg}, 3.00 \mathrm{mmol}, 1.2$ equiv.) in $\mathrm{EtOH}(100 \mathrm{ml})$ was added to form a dark brown solution. The mixture was refluxed for $2 \mathrm{~h}$. The EtOH and residual acetic acid were removed under vacuum before redissolving in a minimum volume of hot $\mathrm{EtOH}$, cooling and recrystallising using cold $\mathrm{Et}_{2} \mathrm{O}$ as an antisolvent. This yielded a very dark brown solid which was isolated by filtration, washed with further cold $\mathrm{Et}_{2} \mathrm{O}$ and dried under vacuum. $739.4 \mathrm{mg}$ of a dark brown powder was obtained (39\% yield).

${ }^{1} \mathrm{H}$ NMR ( $\left.\mathrm{CDCl}_{3}, 500 \mathrm{MHz}\right): \delta$ (all br.) 82.91, 50.13, 30.14, 27.91, 12.65, 9.83, 9.61, 3.83, 2.64, $1.60,0.89,-9.00,71.47$

Crystallised from dichloromethane (DCM) by slow evaporation. Crystal structure obtained, compound data is comparable to literature. ${ }^{[9-11]}$ 




Table S3: Crystal data and structure refinement parameters for 6 .

\begin{tabular}{|l|l|}
\hline Formula & $\mathrm{C} 36 \mathrm{H} 36 \mathrm{Cl} 2 \mathrm{Fe} 3 \mathrm{~N} 4 \mathrm{O} 8$ \\
\hline Molecular mass & 891.14 \\
\hline Temperature & $150 \mathrm{~K}$ \\
\hline Wavelength & $0.71073 \AA$ \\
\hline Crystal system & Triclinic \\
\hline Space group & $\mathrm{P} 1$ \\
\hline Unit cell dimensions & $\mathrm{a}=10.5767(3) \AA \quad \alpha=78.053(3)^{\circ}$ \\
\hline & $\mathrm{b}=13.1865(5) \AA \quad \quad \quad \quad \mathrm{A}=74.294(3)^{\circ}$ \\
\hline & $\mathrm{c}=13.8751(4) \AA \quad \quad \quad \quad \quad=74.789(3)^{\circ}$ \\
\hline Volume & $1792.41(10) \AA 3$ \\
\hline Z & 2 \\
\hline Radiation & MoKa $(\lambda=0.71073)$ \\
\hline Density (calculated) & 1.559 \\
\hline Absorption coefficient & 1.01 \\
\hline F(000) & 860.0 \\
\hline Crystal size & $0.166 \times 0.130 \times 0.050 \mathrm{~mm}$ \\
\hline Theta range for data collection & $3.2290-28.4970^{\circ}$ \\
\hline Index ranges & $-14<=\mathrm{h}<=13 ;-18<=\mathrm{k}<=17 ;-18<=\mathrm{l}<=18$ \\
\hline Reflections collected & 17062 \\
\hline
\end{tabular}




\begin{tabular}{|l|l|}
\hline Independent reflections & $9151[\mathrm{R}(\mathrm{int})=0.0315, \mathrm{R}(\mathrm{sigma})=0.0641]$ \\
\hline Data Completeness & $99.88 \%$ \\
\hline Absorption correction & Multi-scan \\
\hline Max. and min. transmission & $1.00000 / 0.95498$ \\
\hline Refinement method & \\
\hline Final R indices [I>2sigma(I)] & $\mathrm{R} 1=0.0605, \mathrm{wR} 2=0.1496$ \\
\hline R indices (all data) & $\mathrm{R} 1=0.0828$ \\
\hline Largest diff. peak and hole & $0.94 /-1.35$ \\
\hline
\end{tabular}

\section{Spectroscopic Characterisation of Products}

\section{Products}

3a

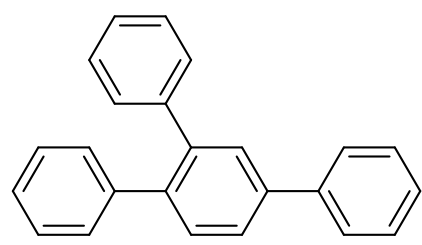

Isolated yield: $97 \%$ Spec. Purity: $100 \% \quad$ Isomeric Ratio of 1,2,4 to 1,3,5: $98: 2$

${ }^{1}{ }_{H}$ NMR $\left(\mathrm{CDCl}_{3}, 500 \mathrm{MHz}\right): \delta 7.82(\mathrm{~s}, 3 \mathrm{H}(1,3,5$ isomer, $0.4 \%))$, 7.72-7.66 (m, 4H), 7.55-7.46 $(\mathrm{m}, 3 \mathrm{H}), 7.42-7.36(\mathrm{~m}, 1 \mathrm{H})$, 7.27-7.18 (m, 10H); ${ }^{13} \mathrm{C} \mathrm{NMR}\left(\mathrm{CDCl}_{3}, 126 \mathrm{MHz}\right): \delta 141.6(\mathrm{C}-\mathrm{Ar})$, 141.3 (C-Ar), 141.1 (C-Ar), 140.7 (C-Ar), 140.5 (C-Ar), 139.7 (C-Ar), 131.2, 130.1, 130.0, 129.6, 129.0, 128.1, 128.0, 127.6, 127.3, 126.7, 126.7, 126.3; HR-MS (ASAP+) [M]: 306.1408 (calcd.), 306.1404 (obs.)

3b



Isolated yield: $65 \%$ Spec. Purity: $100 \% \quad$ Isomeric Ratio of 1,2,4 to 1,3,5: $100: 0$

${ }^{1} \mathrm{H}$ NMR $\left(\mathrm{CDCl}_{3}, 300 \mathrm{MHz}\right): \delta$ 7.69-7.62 (m, 4H), 7.53-7.48 (m, 3H, $\left.\mathrm{CH}_{x}\right)$, 7.27-7.24 (m, 4H), 7.14-7.11 (m, 4H), $1.39(\mathrm{~s}, 9 \mathrm{H}), 1.32(\mathrm{~s}, 9 \mathrm{H}), 1.32(\mathrm{~s}, 9 \mathrm{H}) ;{ }^{13} \mathrm{C} \mathrm{NMR}\left(\mathrm{CDCl}_{3}, 126 \mathrm{MHz}\right): \delta 150.4$ $\left(C^{6}\right), 149.5\left(C^{5}\right), 149.4\left(C^{5}\right), 141.0(C-A r), 140.0(C-A r), 139.4(C-A r), 138.8(C-A r), 138.4(C-A r)$, 
138.0 (C-Ar), 131.1, 129.7, 129.6, 129.4, 126.9, 125.9, 125.8, 124.8, 124.8, $34.7\left(C^{3}\right)$, 34.6 $\left(C^{1}\right), 34.6\left(C^{1}\right), 31.5\left(C^{4}\right), 31.5\left(C^{2}\right)$; HR-MS (ASAP+) [M+H]: 475.3365 (calcd.), 475.3362 (obs.)

$3 c$<smiles>COc1ccc(-c2ccc(-c3ccc(OC)cc3)c(-c3ccc(OC)cc3)c2)cc1</smiles>

Isolated yield: $70 \% \quad$ Spec. Purity: $97 \% \quad$ Isomeric Ratio of 1,2,4 to 1,3,5: $92: 8$

${ }^{1} \mathrm{H}$ NMR (CDCl, $\left.500 \mathrm{MHz}\right): \delta$ 7.61-7.55 (m, 4H), 7.45-7.43 (m, 1H), 7.13-7.09 (m, 4H), 7.00$6.98(\mathrm{~m}, 2 \mathrm{H}), 6.80-6.77(\mathrm{~m}, 4 \mathrm{H}), 3.87(\mathrm{~s}, 9 \mathrm{H}(1,3,5$ isomer, $8 \%)), 3.86(\mathrm{~s}, 3 \mathrm{H}, \mathrm{Mex}), 3.82(\mathrm{~s}, 3 \mathrm{H}$ (SM, 3\%)), 3.80 (s, 3H, Mey), 3.79 (s, 3H, Mey); ${ }^{13} \mathrm{C} \mathrm{NMR} \mathrm{(CDCl} 3,126$ MHz): 159.4 (C-OMex), 158.5 (C-OMe $\left.{ }^{y}\right), 158.4$ (C-OMey), 140.5 (C-Ar), 139.7 (C-Ar), 138.6 (C-Ar), 134.3 (C-Ar), 133.9 (C-Ar), 133.4 (C-Ar), 131.1, 131.1, 131.0, 129.1, 128.2, 125.5, 114.4, 113.6, 113.6, $55.5\left(\mathrm{Me}^{\mathrm{x}}\right)$, $55.3\left(\mathrm{Me}^{\mathrm{y}}\right), 55.3\left(\mathrm{Me}^{\mathrm{y}}\right)$; HR-MS (ASAP+) [M+H]: 397.1804 (calcd.), 397.1805 (obs.)

3d<smiles>Fc1ccc(-c2ccc(-c3ccc(F)cc3)c(-c3ccc(F)cc3)c2)cc1</smiles>

Isolated yield: $90 \% \quad$ Spec. Purity: $99 \% \quad$ Isomeric Ratio of 1,2,4 to 1,3,5: $98: 2$

${ }^{1} \mathrm{H}$ NMR $\left(\mathrm{CDCl}_{3}, 500 \mathrm{MHz}\right): \delta$ 7.63-7.56 (m, 4H), 7.47-7.45 (m, 1H), 7.17-7.10 (m, 6H), 6.97-


$\left.C_{a}\right), 162.0\left(d, J=246.2 \mathrm{~Hz}, C_{a}\right), 140.2,139.8,138.7,137.3$ (d, J = 3.3 Hz), 136.9 (d, J = 3.4 Hz), $136.6(d, J=3.4 \mathrm{~Hz}), 131.5(\mathrm{dd}, J=8.0 \mathrm{~Hz}, 4.9 \mathrm{~Hz}), 131.2,129.3,129.1(1,3,5), 129.0(1,3,5)$, 128.9, 128.8, 126.3, 115.9 (d, J = 21.5 Hz, $\left.C_{d}\right), 115.2$ (d, J = 21.4 Hz, $\left.C_{b}\right), 115.2(d, J=21.4 \mathrm{~Hz}$, $\left.\mathrm{C}_{\mathrm{b}}\right) ;{ }^{19} \mathrm{~F}$ NMR (CDCl $\left.3,470 \mathrm{MHz}\right): \delta$-112.44 (m, (SM, 1 \%)), -115.05 (m, (1,3,5 isomer, $\left.\left.2 \%\right)\right)$, 115.22 (m, 1F), -155.69 (m, 1F), -155.81 (m, 1F); HR-MS (ASAP+) [M]: 360.1126 (calcd.), 360.1130 (obs.) 
3e<smiles>Cc1cccc(-c2ccc(-c3cccc(C)c3)c(-c3cccc(C)c3)c2)c1</smiles>

Isolated yield: $87 \% \quad$ Spec. Purity: $98 \% \quad$ Isomeric Ratio of 1,2,4 to 1,3,5: $95: 5$

${ }^{1} \mathrm{H}$ NMR $\left(\mathrm{CDCl}_{3}, \mathbf{5 0 0} \mathrm{MHz}\right): \delta$ 7.67-7.64 (m, 2H), 7.52-7.49 (m, 3H), 7.38-7.35 (m, 1H), 7.21$7.19(\mathrm{~m}, 1 \mathrm{H}), 7.13-7.09(\mathrm{~m}, 3 \mathrm{H}), 7.07-7.03(\mathrm{~m}, 3 \mathrm{H}), 6.97-6.94(\mathrm{~m}, 2 \mathrm{H}), 2.47(\mathrm{~s}, 3 \mathrm{H}(1,3,5$ isomer, $5 \%)) 2.45\left(\mathrm{~s}, 3 \mathrm{H}, \mathrm{Me}^{\mathrm{x}}\right), 2.35(\mathrm{~s}, 3 \mathrm{H}(\mathrm{SM}, 2 \%)), 2.30\left(\mathrm{~s}, 3 \mathrm{H}, \mathrm{Me}^{\mathrm{y}}\right), 2.30\left(\mathrm{~s}, 3 \mathrm{H}, \mathrm{Me}^{\mathrm{y}}\right) ;{ }^{13} \mathrm{C}$ NMR (CDCl, 126 MHz): $\delta 141.6$ (C-Ar), 141.3 (C-Ar), 141.1 (C-Ar), 140.9 (C-Ar), 140.4 (C-Ar),

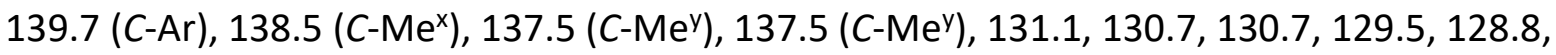
128.2, 128.1, 127.8, 127.7, 127.4, 127.3, 127.2, 127.2, 126.1, 124.4, $21.7\left(\mathrm{Me}^{\mathrm{x}}\right), 21.5\left(\mathrm{Me}^{\mathrm{y}}\right)$; FTIR (ATR): 3026 ( $\left.\mathrm{C}_{\mathrm{Ar}}-\mathrm{H}\right), 2919\left(\mathrm{C}-\mathrm{H}_{3}\right), 2859\left(\mathrm{C}-\mathrm{H}_{3}\right), 1603,1583,1553,1474,1378,1168$, 1142, 1094, 1036, 1000, 881, 855, 833, 759, 779, $703 \mathrm{~cm}^{-1}$; HR-MS (ASAP+) [M]: 348.1878 (calcd.), 348.1874 (obs.)

$3 f$<smiles>Nc1ccccc1-c1ccc(-c2ccccc2N)c(-c2ccccc2N)c1</smiles>

Isolated yield: $42 \%$ Spec. Purity: $100 \%$ Isomeric Ratio of 1,2,4 to 1,3,5: $100: 0$

NB. Std. HBPin concentration insufficient due to consumption by amine. 3 eq. HBPin used to ensure reaction, and secondary column performed to remove degradation products on silica (40 EtOAc : 60 Pet. ether).

${ }^{1}{ }_{\mathrm{H}} \mathrm{NMR}\left(\mathrm{CDCl}_{3}, \mathbf{5 0 0} \mathrm{MHz}\right): \delta$ 7.58-7.52 (m, 3H), 7.22-7.16 (m, 2H), 7.05-7.00 (m, 2H), 6.94$6.93(\mathrm{~m}, 2 \mathrm{H}), 6.87-6.84(\mathrm{~m}, 1 \mathrm{H}), 6.80-6.78(\mathrm{~m}, 1 \mathrm{H}), 6.63-6.60(\mathrm{~m}, 4 \mathrm{H}) ;{ }^{13} \mathrm{C} \mathrm{NMR}\left(\mathrm{CDCl}_{3}, 75\right.$ MHz): $\delta 143.7,143.6,139.4,131.6,130.7,128.8,128.5,126.9,119.0,118.4,118.4,116.0$, 115.8; FTIR (ATR): 3451 (N-H), 3363 (N-H), 3023 ( $\left.\mathrm{C}_{\mathrm{Ar}}-\mathrm{H}\right)$, 1613, 1577, 1494, 1476, 1449, 1386, $1296,1259,1157,1143,1053,1007,907,852,829,747,728,679 \mathrm{~cm}^{-1}$; HR-MS (NSI+) [M+H]: 352.1808 (calcd.), 352.1810 (obs.) 
$3 g$<smiles>Clc1ccccc1-c1ccc(-c2ccccc2Cl)c(-c2ccccc2Cl)c1</smiles>

Isolated yield: $81 \% \quad$ Spec. Purity: $100 \% \quad$ Isomeric Ratio of 1,2,4 to 1,3,5: $100: 0$

${ }^{1} \mathrm{H}$ NMR (CDCl $\left.3,500 \mathrm{MHz}\right): \delta 7.60$ (s, (1,3,5 isomer, 0.4 \%), 7.57-7.46 (m, 5H), 7.36-7.28 (m,


138.5 (C-Ar), 138.4 (C-Ar), 137.9 (C-Ar), 133.7, 133,5, 133.3, 133.2, 132.7, 132.5, 132.1, 131.9, 131.7, 130.4, 130.2, 129.8, 129.2, 128.8, 128.6, 127.0, 126.3, 126.0; FTIR (ATR): 3057 ( $\left.\mathrm{C}_{\mathrm{Ar}} \mathrm{-H}\right), 1592,1566,1475,1459,1432,1389,1348,1320,1160,1127,1074,1064,1035$, 1005, 945, 906, 865, 835, 751, 735, 694, 676, $660 \mathrm{~cm}^{-1}$; HR-MS (ASAP+) [M+NH $\mathbf{N H}_{4}$ : 426.0583 (calcd.), 426.0587 (obs.)

$3 h$<smiles>c1cncc(-c2ccc(-c3cccnc3)c(-c3cccnc3)c2)c1</smiles>

Isolated yield: $16 \%$ Spec. Purity: $70 \% \quad$ Isomeric Ratio of 1,2,4 to 1,3,5: $100: 0$

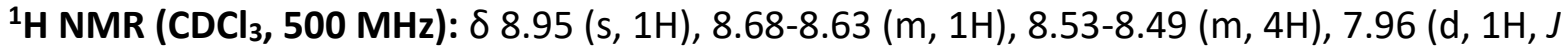
$=7.7 \mathrm{~Hz}), 7.74-7.73(\mathrm{~m}, 1 \mathrm{H}), 7.66(\mathrm{~s}, 1 \mathrm{H}), 7.59-7.58(\mathrm{~m}, 1 \mathrm{H}), 7.46-7.42(\mathrm{~m}, 3 \mathrm{H}), 7.21-7.18(\mathrm{~m}$,


126 MHz): $\delta 150.3$ (C-Ar), 150.3 (C-Ar), 149.1 (C-Ar), 148.4 (C-Ar), 148.3 (C-Ar), 148.3 (C-Ar), 138.3, 138.0, 137.1, 137.0, 137.0, 134.4, 131.6, 129.5, 127.2, 123.7, 123.0, 83.0 (pinacol alcohol $\mathrm{C}-\mathrm{CH}_{3}$ ), 24.9 (pinacol alcohol C- $\mathrm{CH}_{3}$ ), 24.8 (pinacol alcohol C- $\mathrm{CH}_{3}$ ), 24.6 (pinacol alcohol C-CH $\mathrm{CH}_{3}$ ); FTIR (ATR): $3029\left(\mathrm{C}_{\mathrm{Ar}}-\mathrm{H}\right), 2977\left(\mathrm{C}_{\mathrm{Ar}}-\mathrm{H}\right), 2926\left(\mathrm{C}_{\mathrm{Ar}}-\mathrm{H}\right), 1589,1567,1468,1454$, 1406, 1378, 1329, 1270, 1188, 1104, 1049, 1024, 1003, 923, 894, 850, 805, 757, 710, 676 $\mathrm{cm}^{-1}$; HR-MS (NSI+) [M]: 310.1339 (calcd.), 310.1340 (obs.) 




Isolated yield: $81 \%$ Spec. Purity: $100 \% \quad$ Isomeric Ratio of 1,2,4 to 1,3,5: $97: 3$

${ }^{1} \mathrm{H}_{\mathrm{NMR}}\left(\mathrm{CDCl}_{3}, 500 \mathrm{MHz}\right): \delta 8.85$ (s, (1,3,5 isomer, $\left.3 \%\right), 8.39\left(\mathrm{~s}, 1 \mathrm{H}, \mathrm{C}^{4}-\mathrm{H}\right), 8.19$ (d, 1H, $\mathrm{C}^{1}-\mathrm{H}$, $J=8.0 \mathrm{~Hz}), 7.75\left(\mathrm{~d}, 1 \mathrm{H}, \mathrm{C}^{2}-\mathrm{H}, J=7.9 \mathrm{~Hz}\right), 4.46-4.37\left(\mathrm{~m}, 6 \mathrm{H}, \mathrm{C}^{5}-\mathrm{H}_{2}\right), 1.43-1.36\left(\mathrm{~m}, 9 \mathrm{H}, \mathrm{C}^{6}-\mathrm{H}_{3}\right)$; ${ }^{13} \mathrm{C} \mathrm{NMR}\left(\mathrm{CDCl}_{3}, 126 \mathrm{MHz}\right): \delta 167.1\left(\mathrm{C}^{3}\right), 166.6\left(\mathrm{C}^{3}\right), 165.1$ (1,3,5 isomer, $\left.\operatorname{Ar}-\mathrm{C}-\mathrm{H}\right), 165.0\left(\mathrm{C}^{3}\right)$, $136.2\left(C^{1,2,4}\right), 134.4(1,3,5$ isomer, $\operatorname{Ar}-C-R), 132.7\left(C^{1,2,4}\right), 132.1\left(C^{1,2,4}\right), 132.0(C=0), 130.1$ $(C=0), 128.8(C=0), 62.0\left(C^{5}\right), 61.9\left(C^{5}\right), 61.7\left(C^{5}\right), 14.2\left(C^{6}\right), 14.1\left(C^{6}\right), 14.0\left(C^{6}\right)$;HR-MS (ASAP+) [M+H]: 295.1182 (calcd.), 295.1180 (obs.)

3j


Isolated yield: $72 \% \quad$ Spec. Purity: $100 \% \quad$ Isomeric Ratio of 1,2,4 to 1,3,5: $56: 44$

${ }^{1}{ }_{H}$ NMR $\left(\mathrm{CDCl}_{3}, 500 \mathrm{MHz}\right): \delta 7.05\left(\mathrm{~d}, 1 \mathrm{H}, \mathrm{C}^{5}-\mathrm{H}\right), 6.97-6.92\left(\mathrm{~m}, 2 \mathrm{H}, \mathrm{C}^{2,3}-\mathrm{H}\right), 6.82(\mathrm{~s},(1,3,5$ isomer, $\mathrm{C}^{8}-\mathrm{H}, 44$ \%)) 2.59-2.50 (m, $\left.\mathrm{CH}_{2}\right), 1.64-1.54\left(\mathrm{~m}, \mathrm{CH}_{2}\right), 1.38-1.20\left(\mathrm{~m}, \mathrm{CH}_{2}\right), 0.93-0.88(\mathrm{~m}$, $\left.\mathrm{CH}_{3}\right) ;{ }^{13} \mathrm{C}$ NMR $\left(\mathrm{CDCl}_{3}, 126 \mathrm{MHz}\right): \delta 142.8\left(\mathrm{C}^{7}\right), 140.5\left(\mathrm{C}^{1,4,6}\right), 140.3\left(\mathrm{C}^{1,4,6}\right), 137.8\left(\mathrm{C}^{1,4,6}\right), 129.4$ $\left(C^{2,3,5}\right), 129.1\left(C^{2,3,5}\right), 126.0\left(C^{8}\right), 125.8\left(C^{2,3,5}\right), 36.1,35.7,32.9,32.5,32.2,31.8,31.8,31.4$, 31.4, 31.2, 24.9, 22.75, 22.7, 14.2; HR-MS (ASAP+) [M-H]: 287.2739 (calcd.), 287.2737 (obs.)

$3 \mathbf{k}$

Isolated yield after $1 \mathrm{~h}: 8 \%$ Spec. Purity: $100 \%$ Isomeric Ratio of 1,2,4 to 1,3,5: $56: 44$

Spec yield after $10 \mathrm{~h}: 27 \%$

Isomeric Ratio of 1,2,4 to 1,3,5: $56: 44$

${ }^{1}{ }_{H}$ NMR $\left(\mathrm{CDCl}_{3}, \mathbf{5 0 0} \mathrm{MHz}\right): \delta$ 7.05-7.04 (m, $\left.1 \mathrm{H}, \mathrm{C}^{5}-\mathrm{H}\right)$, 6.95-6.92 (m, 2H, $\left.\mathrm{C}^{2,3}-\mathrm{H}\right), 6.81(\mathrm{~s},(1,3,5$ isomer, $\left.\left.\mathrm{C}^{8}-\mathrm{H}, 44 \%\right)\right)$, alkyl 1,2,4 not resolvable from 1,3,5; 2.57-2.51 (m, $\left.\mathrm{CH}_{2}\right), 1.66-1.58(\mathrm{~m}$, $\left.\mathrm{CH}_{2}\right), 1.00-0.92\left(\mathrm{~m}, \mathrm{CH}_{3}\right)$. Data comparable to previous reports in the literature. ${ }^{[12]}$

3I<smiles>C#CCc1ccccc1</smiles>


Isolated yield after $1 \mathrm{~h}: 4 \% \quad$ Spec. Purity: $67 \% \quad$ Isomeric Ratio of 1,2,4 to 1,3,5: $67: 33$

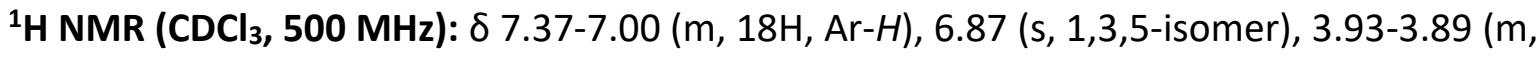
$6 \mathrm{H}, \mathrm{CH}_{2}$ ), 3.62 (d, 1,3,5-isomer, 33\%). Data comparable to previous reports in the literature. ${ }^{[12]}$

\section{Kinetic Studies}

Kinetic studies were undertaken using the general method for cyclotrimerisation of alkynes (section 2) while varying the levels of catalyst and substrate appropriately. The substrate used was 1-(tert-butyl)-4-ethynylbenzene ('BuPA), which undergoes cyclotrimerization to form product $\mathbf{3 b}$. Reactions monitored by consumption of substrate alkynyl proton vs. a trimethoxybenzene (TMB) internal standard using in-situ NMR analysis $\left({ }^{1} \mathrm{H} N M R, \mathrm{CD}_{3} \mathrm{CN}, 500\right.$ $\mathrm{MHz})$. 




Figure S1: Example of kinetics NMR spectra obtained in situ



Figure S2: Example reaction profile obtained using standard reaction conditions as stipulated in general method for cyclotrimerization of alkynes. Rate taken from $\sim 40-60$ minutes linear region. 


\section{Kinetics with respect to catalyst:}

Reaction profiles obtained at of $0.5 \mathrm{~mol} \%, 0.625 \mathrm{~mol} \%, 0.75 \mathrm{~mol} \%, 0.875 \mathrm{~mol} \%$ and $1 \mathrm{~mol} \%$ of $1.0 .25 \mathrm{mmol}$ of ${ }^{\mathrm{B}} \mathrm{BuPA}$ used throughout.

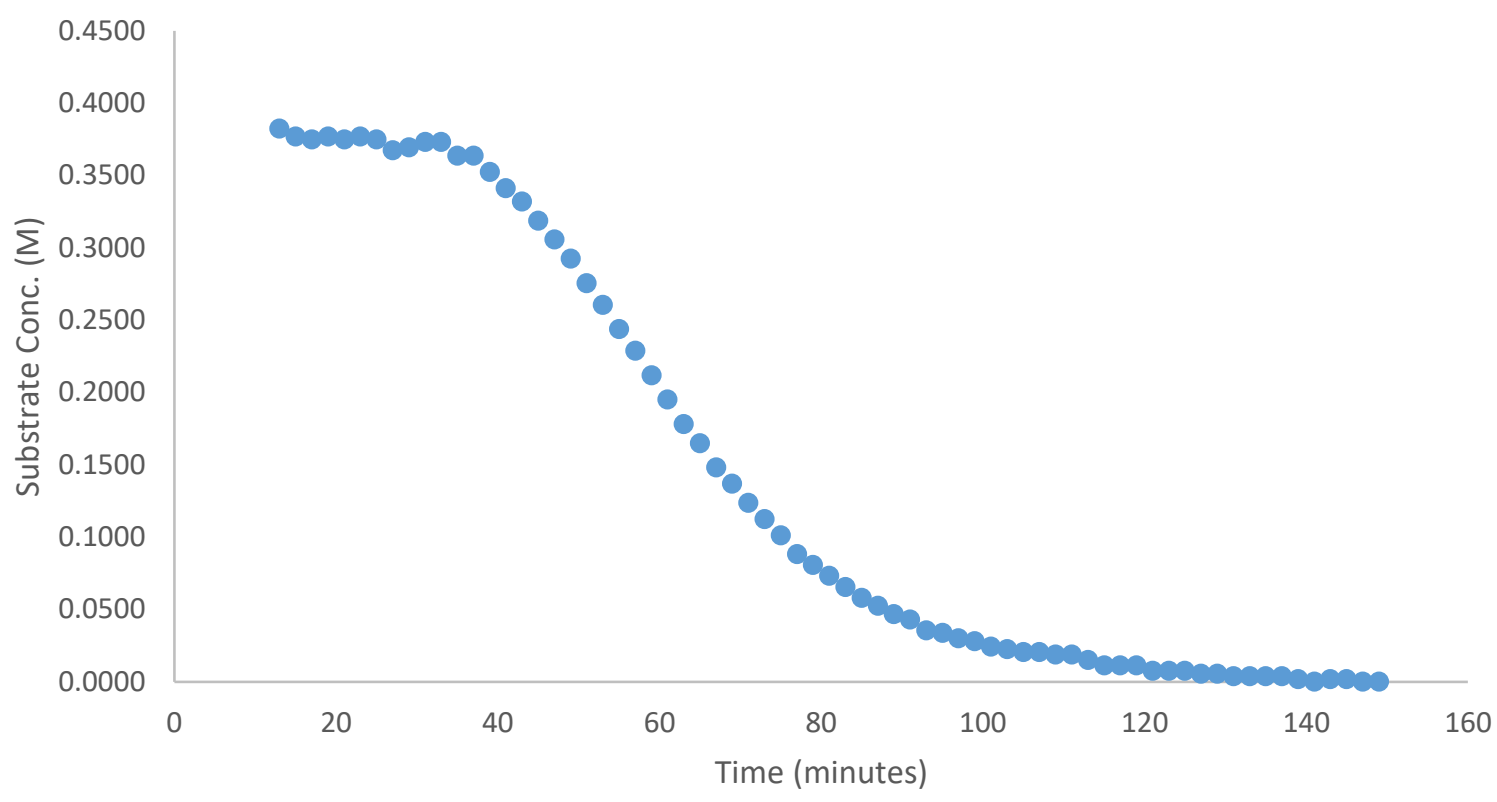

Figure S3: Reaction profile obtained using $0.5 \mathrm{~mol} \% \mathrm{Fe}$, substrate ${ }^{\mathrm{t} B u P A}$



Figure S4: Reaction profile obtained using 0.625 mol\% Fe, substrate ${ }^{\text {B }}$ uPA 
conc. SM

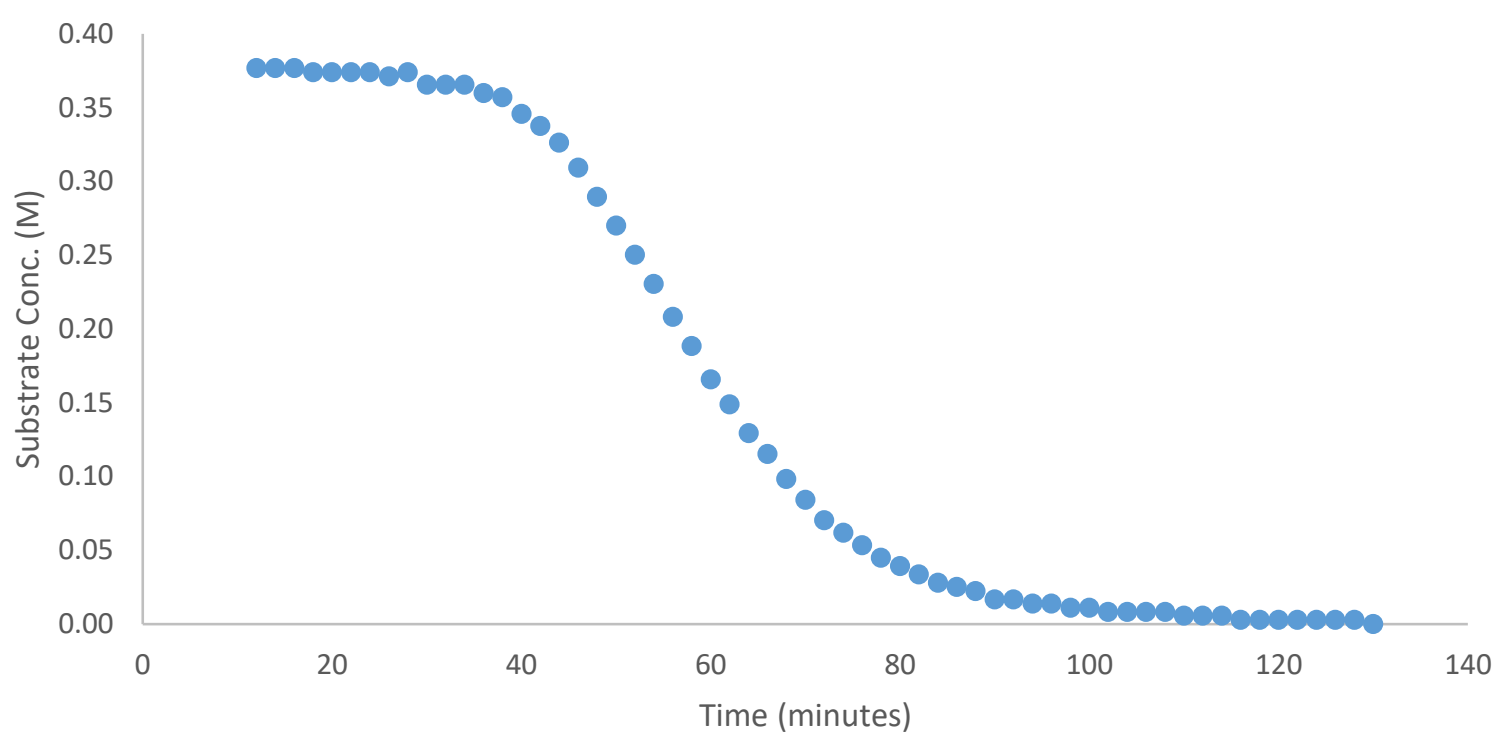

Figure S5: Reaction profile obtained using $0.75 \mathrm{~mol} \% \mathrm{Fe}$, substrate ${ }^{\mathrm{t}} \mathrm{BuPA}$

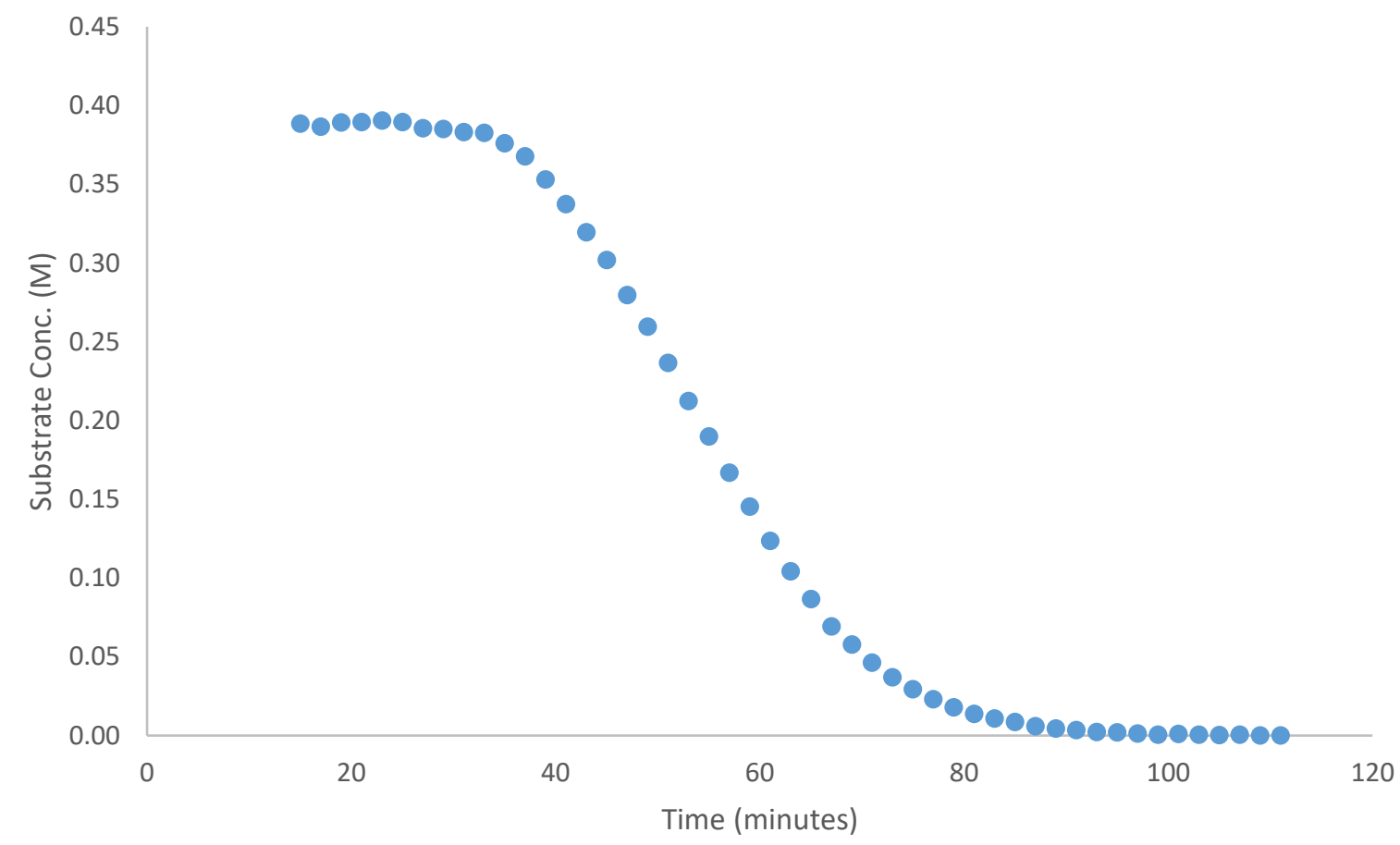

Figure S6: Reaction profile obtained using $0.875 \mathrm{~mol} \% \mathrm{Fe}$, substrate ${ }^{\mathrm{t}} \mathrm{BuPA}$ 


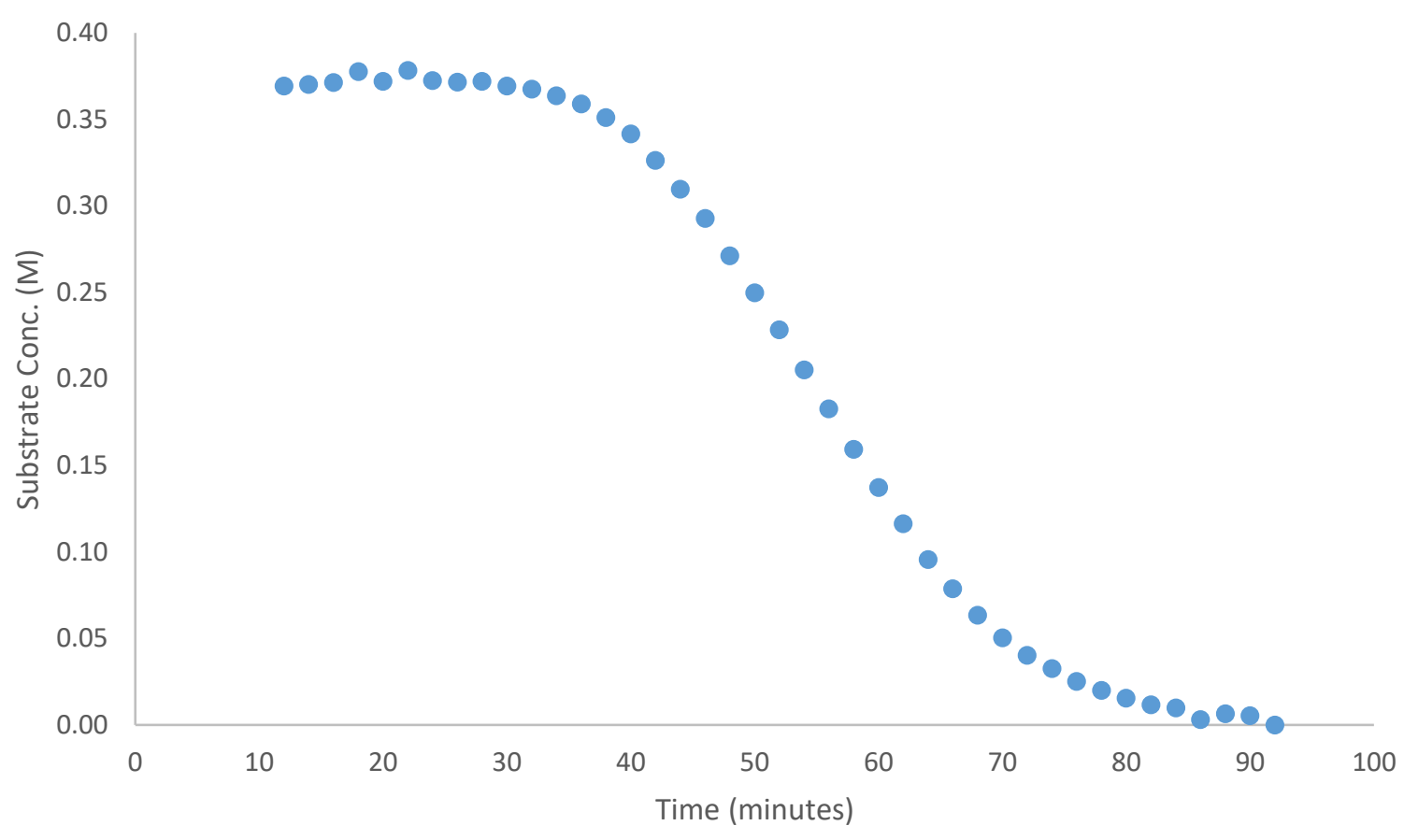

Figure S7: Reaction profile obtained using 1 mol\% Fe, substrate ${ }^{\text {tBuPA }}$

Functions of catalyst concentration was plotted against rate of reaction at 1 loadings of 0.5 mol\%, 0.625 mol\%, 0.75 mol\%, 0.875 mol\% and 1 mol\%: Criteria for fit is an $R^{2}>0.995$ when origin is plotted as a point.

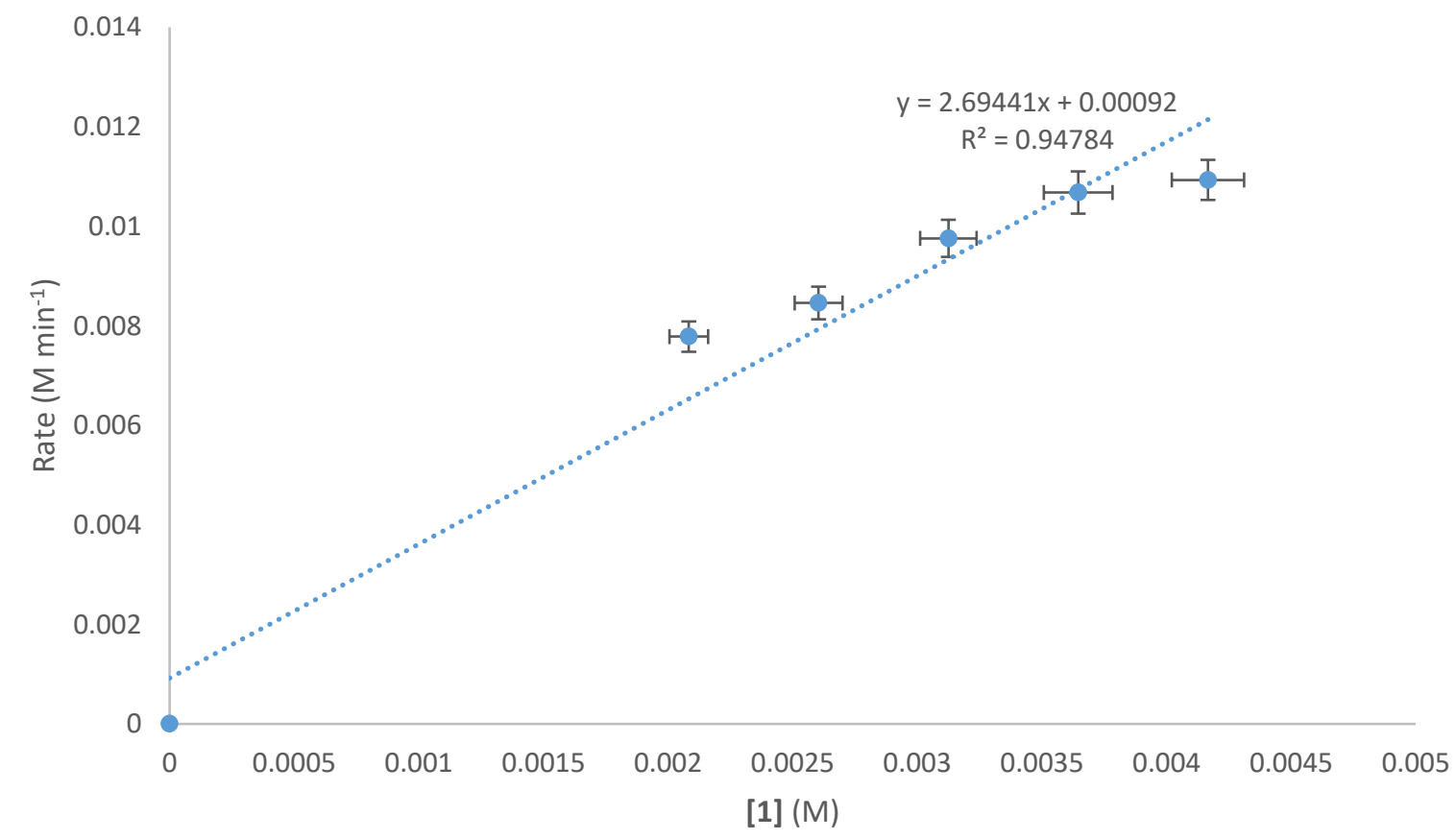

Figure S8: $[1]^{\wedge} 1$ vs rate. $R^{2}<0.995$, therefore not $1^{\text {st }}$ order wrt. to catalyst. 


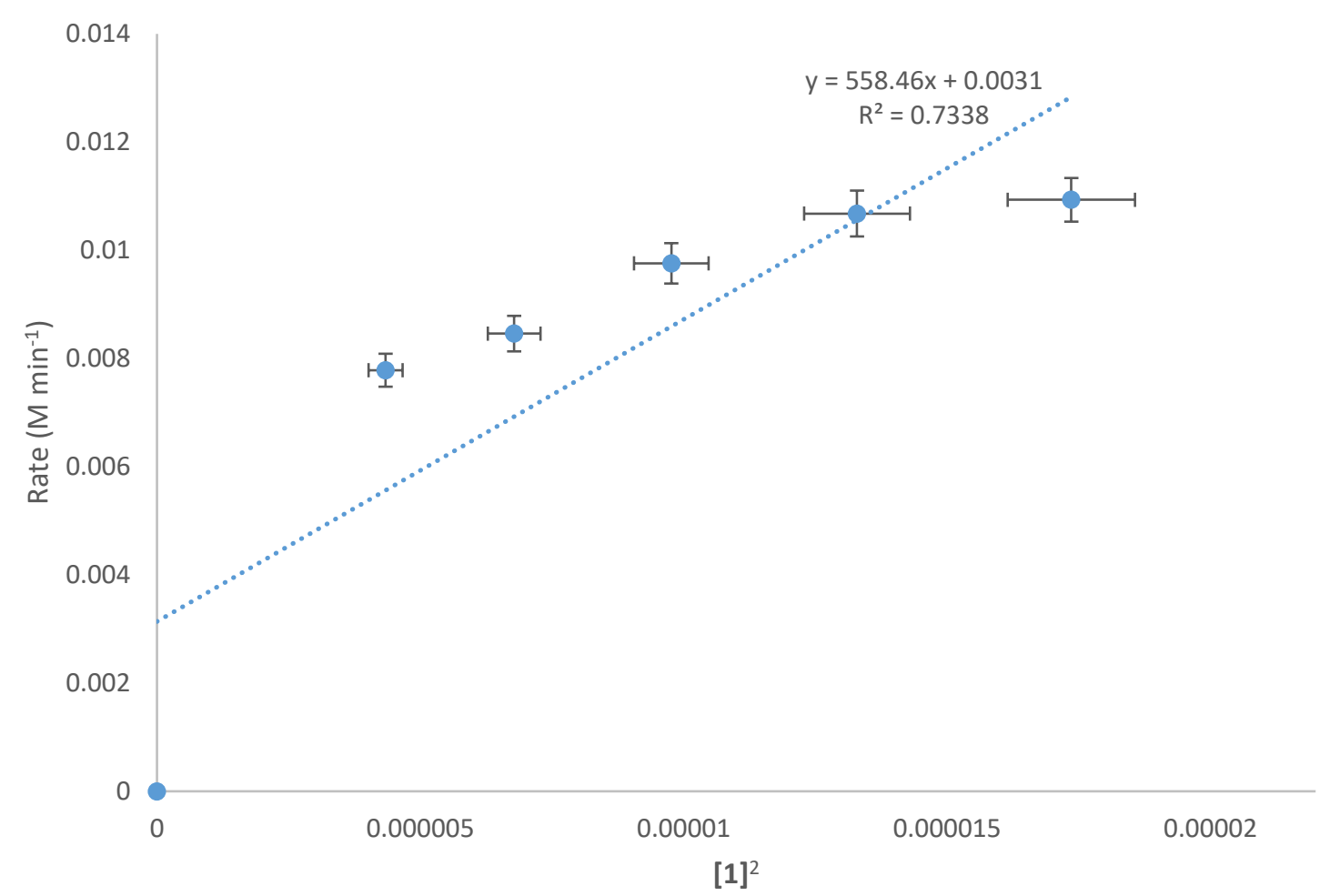

Figure S9: $[1]^{\wedge} 2$ vs rate. $R^{2}<0.995$, therefore not $2^{\text {nd }}$ order wrt. catalyst.



Figure S10: $[1]^{\wedge} 0.5$ vs rate. $R^{2}>0.995$, therefore reaction is 0.5 order wrt. catalyst.

\section{Kinetics with respect to substrate:}


Reaction profiles obtained at $0.25 \mathrm{mmol}, 0.50 \mathrm{mmol}, 0.75 \mathrm{mmol}$ and $1.00 \mathrm{mmol}$ of tBuPA, with 1 mol\% of 1.

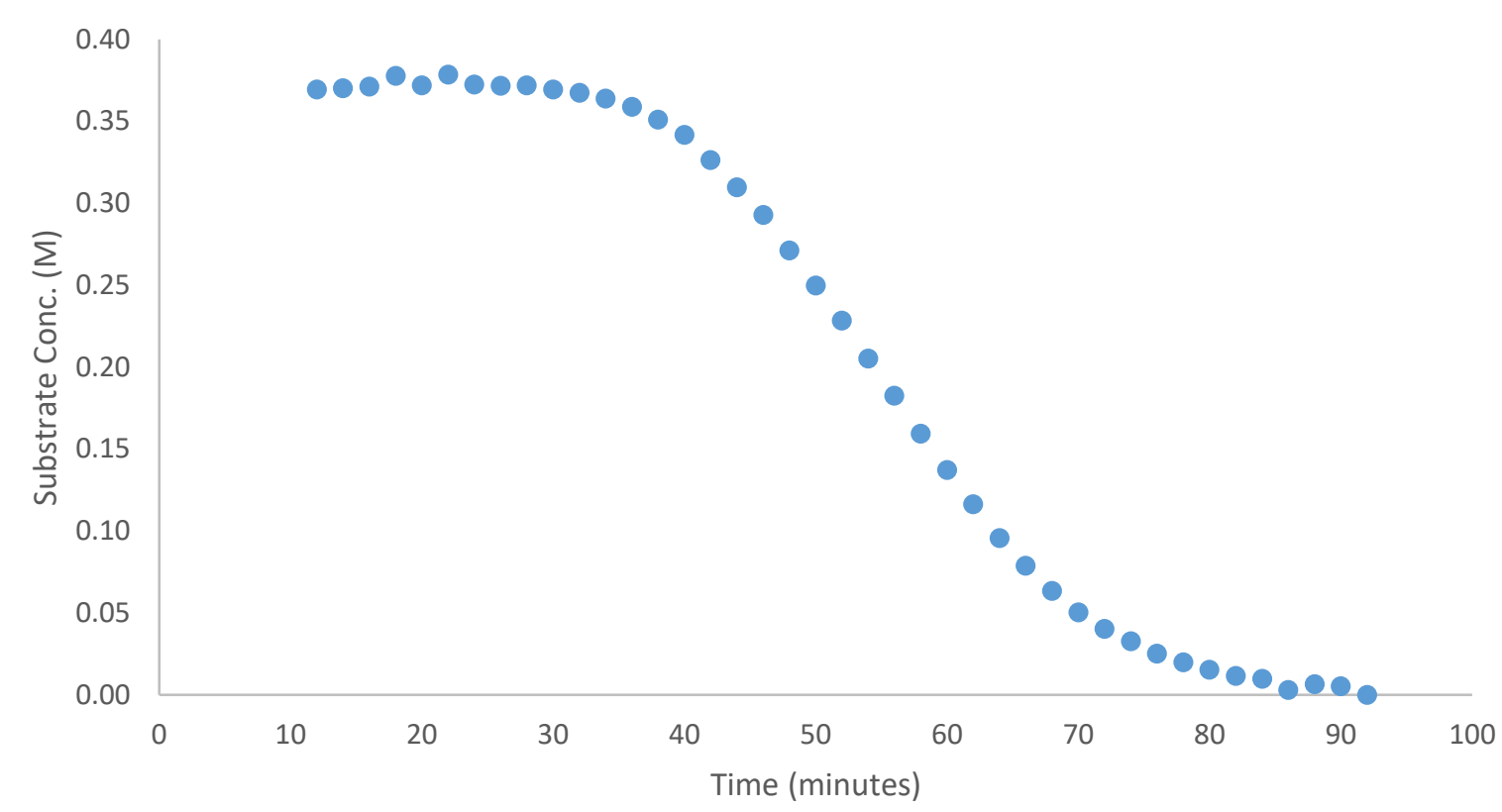

Figure S11: Reaction profile obtained using $0.25 \mathrm{mmol}$ tBuPA, 1 mol\% 1.

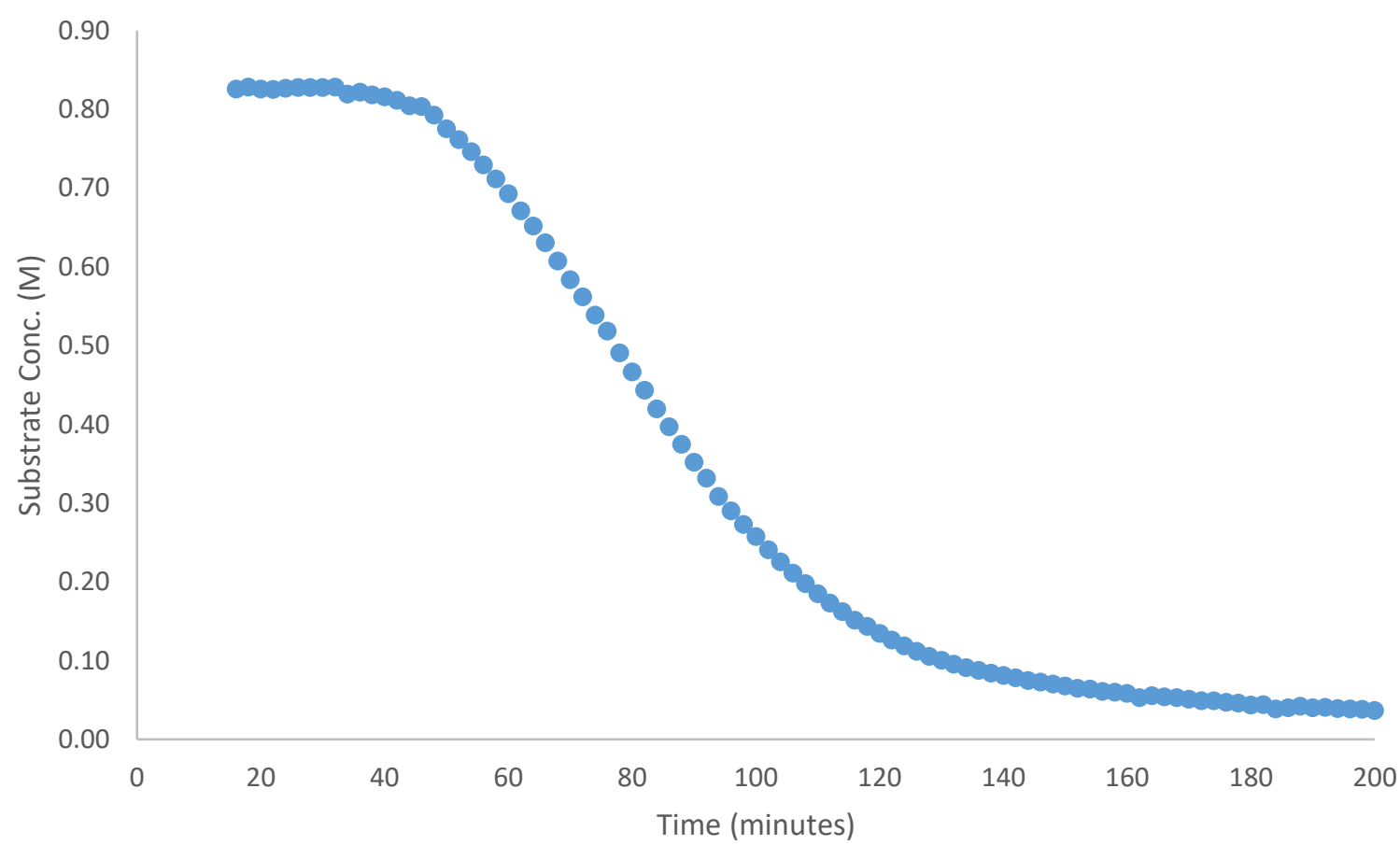

Figure S12: Reaction profile obtained using $0.50 \mathrm{mmol}$ tBuPA, 1 mol\% 1. 


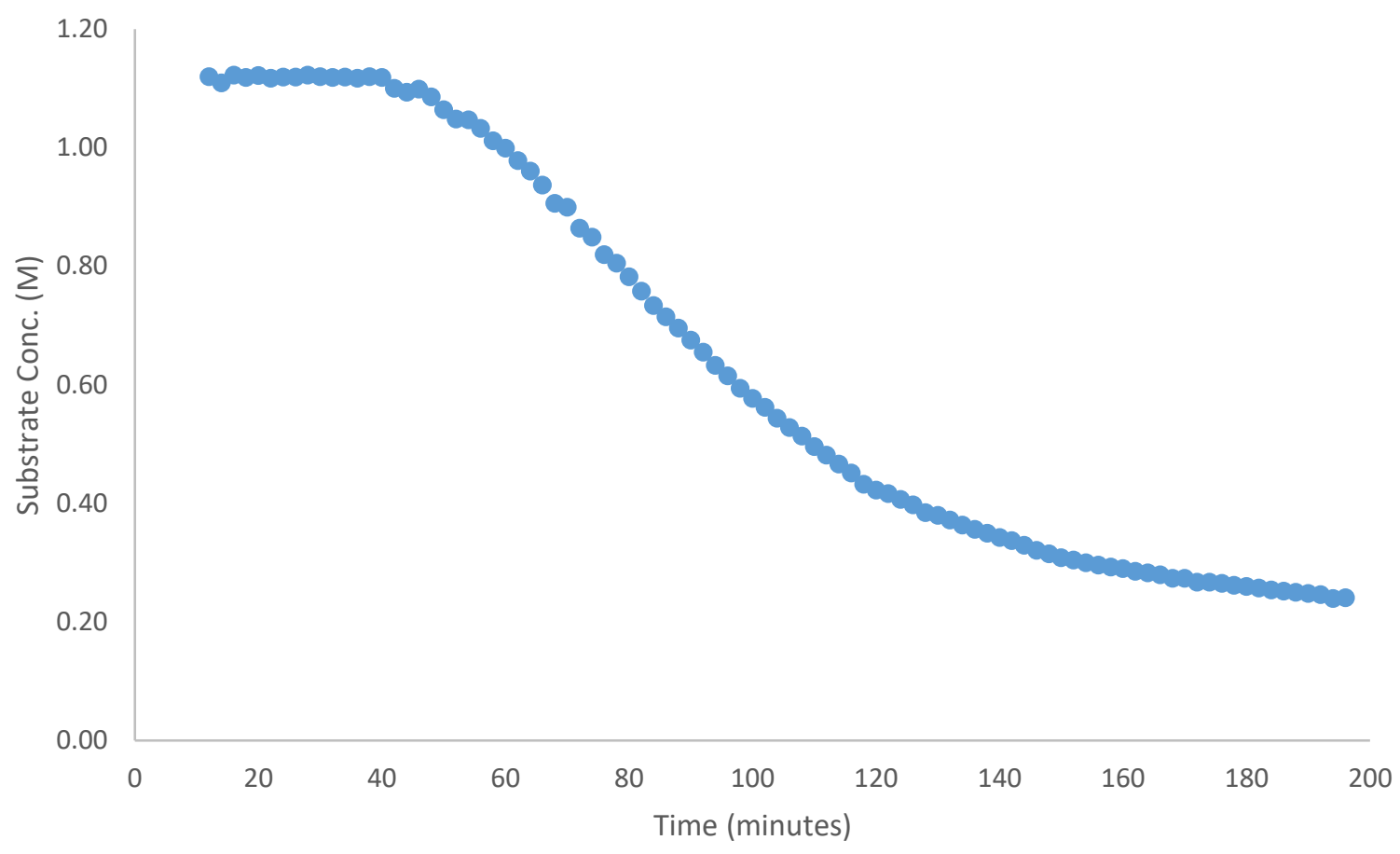

Figure S13: Reaction profile obtained using $0.75 \mathrm{mmol}$ tBuPA, 1 mol\% 1.

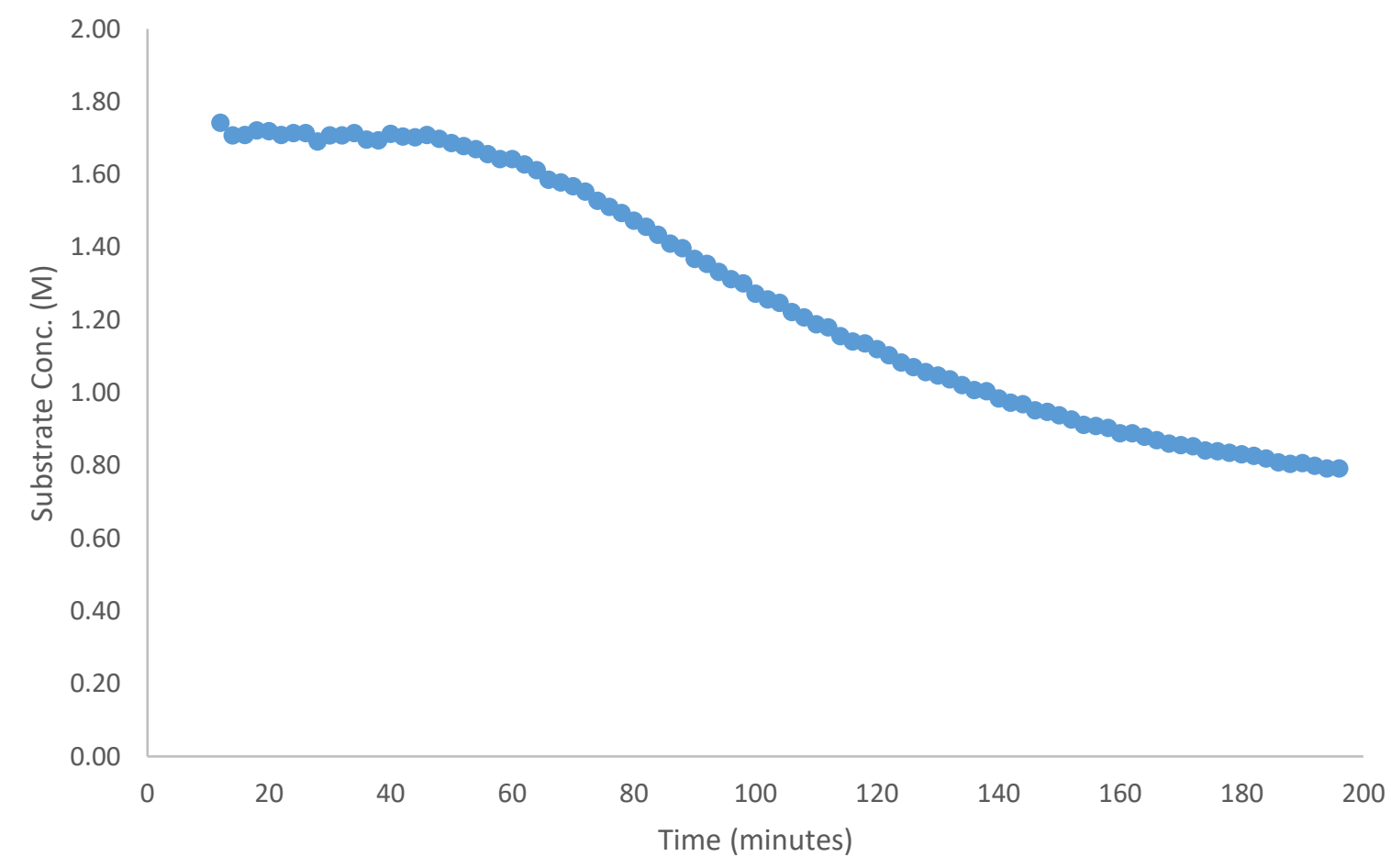

Figure S14: Reaction profile obtained using $1.00 \mathrm{mmol}$ tBuPA, 1 mol\% 1. 
Functions of catalyst concentration was plotted against rate of reaction at catalyst loadings of $0.25 \mathrm{mmol}, 0.50 \mathrm{mmol}, 0.75 \mathrm{mmol}$ and $1.00 \mathrm{mmol}$ : Criteria for fit is an $\mathrm{R}^{2}>0.995$ when origin is plotted as a point.

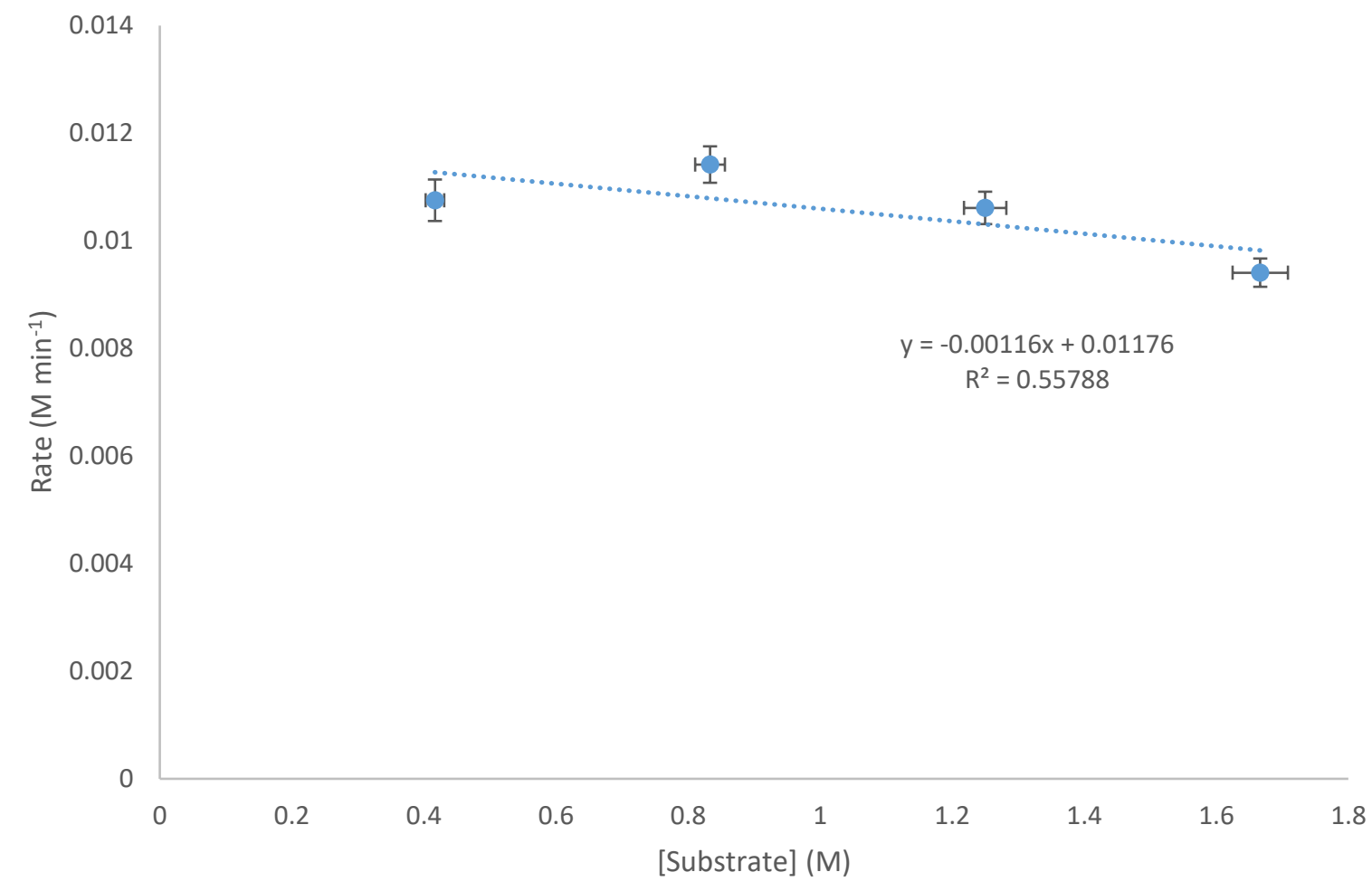

Figure S15: [Substrate] vs rate. Reasonably linear trend which does not go through origin allows tentative assignment of zero order wrt. substrate.

\section{Control and catalyst inhibition experiments:}

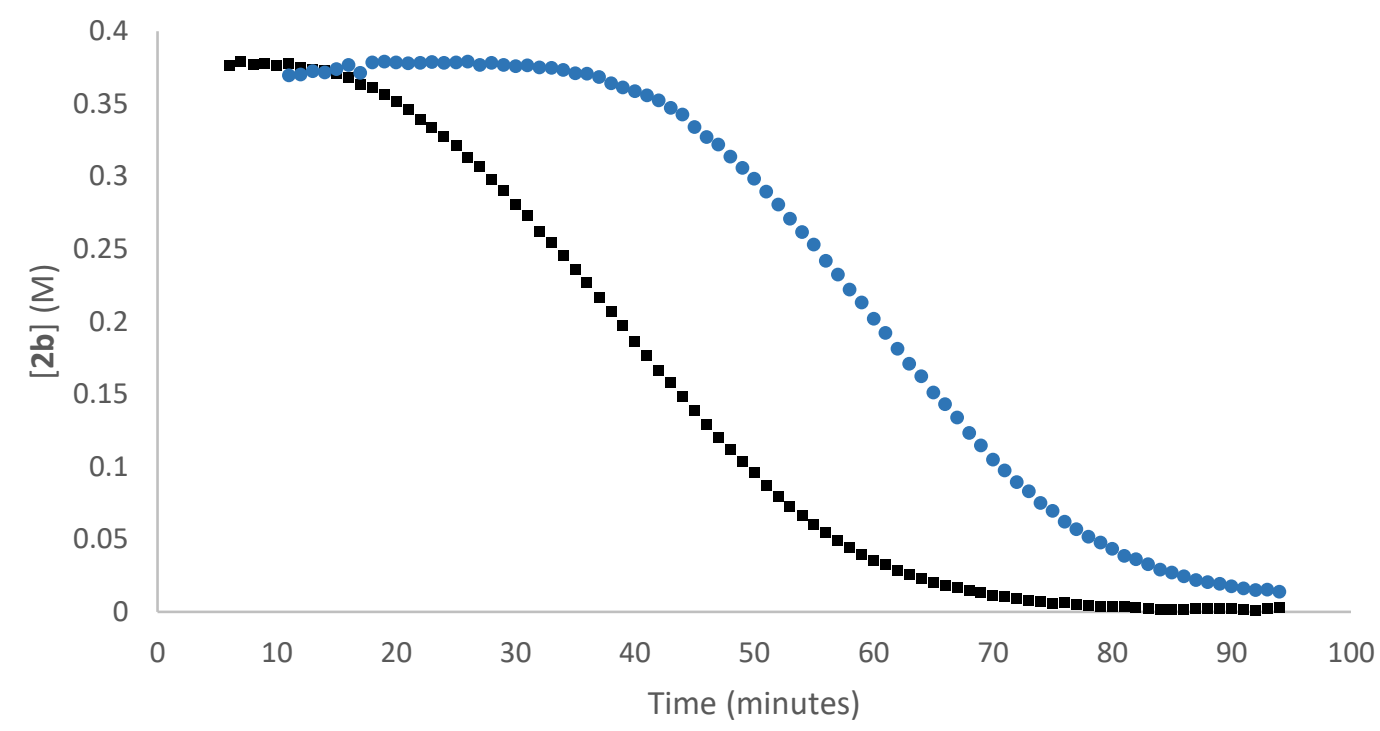


Figure S16: Reaction profile of concentration of $\mathbf{2 b}(\bullet)$ over time under standard conditions, and mixing of $\mathbf{1}$ and HBpin for 20 mins before addition of $\mathbf{2 b}(\mathbf{a})$

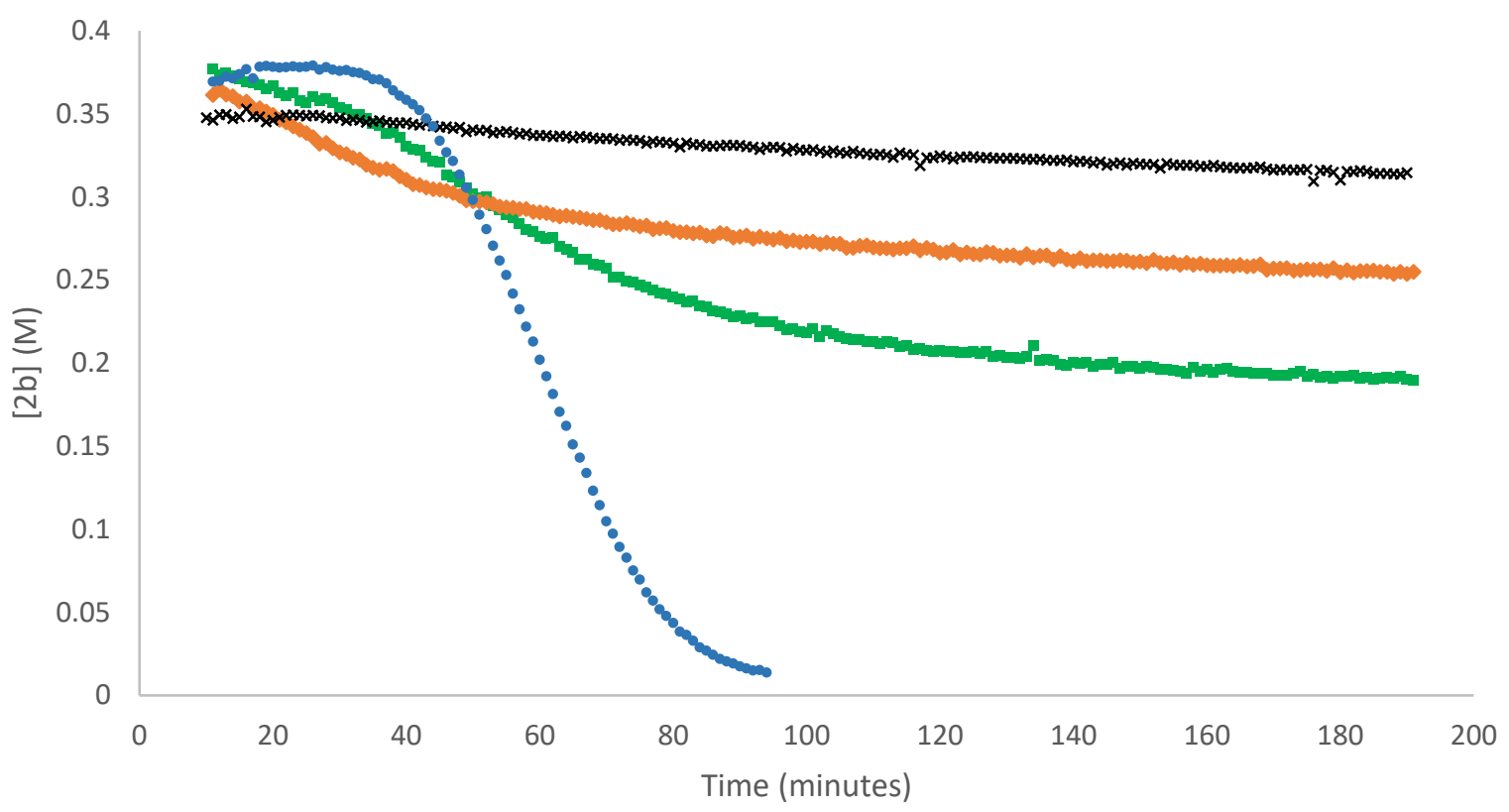

Figure S17: Reaction profile of concentration of $\mathbf{2 b}(\mathbf{O})$ over time under standard conditions, and with $\mathrm{PMe}_{3}$ additive $1 \mathrm{~mol} \%$ ( $\square$ ), $5 \mathrm{~mol} \%(\diamond)$ and, $10 \mathrm{~mol} \%$ (X).

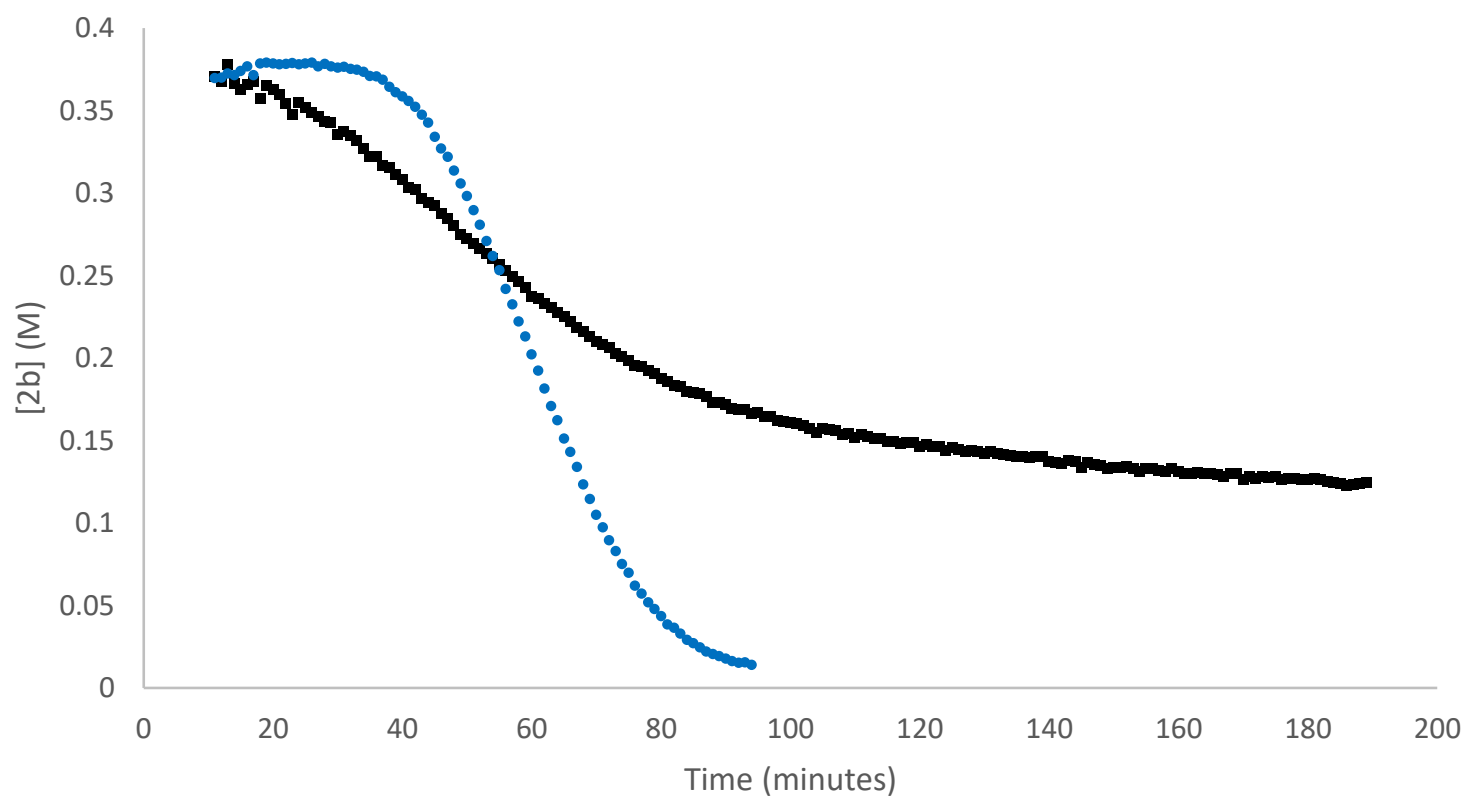

Figure S18: Concentration of $\mathbf{2 b}(\Theta)$ over time under standard conditions, and mixing of $\mathbf{1}$ and HBpin for 20 mins before addition of $\mathbf{2 b}$ and $\mathrm{PMe}_{3}$ (0.5 mol\%) ( $\left.\boldsymbol{\square}\right)$. 


\section{Structure Distortion Quantification Examples}

1 distortion (geometry from crystal structure). Angles measured C23-C10-C34-C43 and C57C66-C47-C56. Average distortion $11^{\circ}$ :

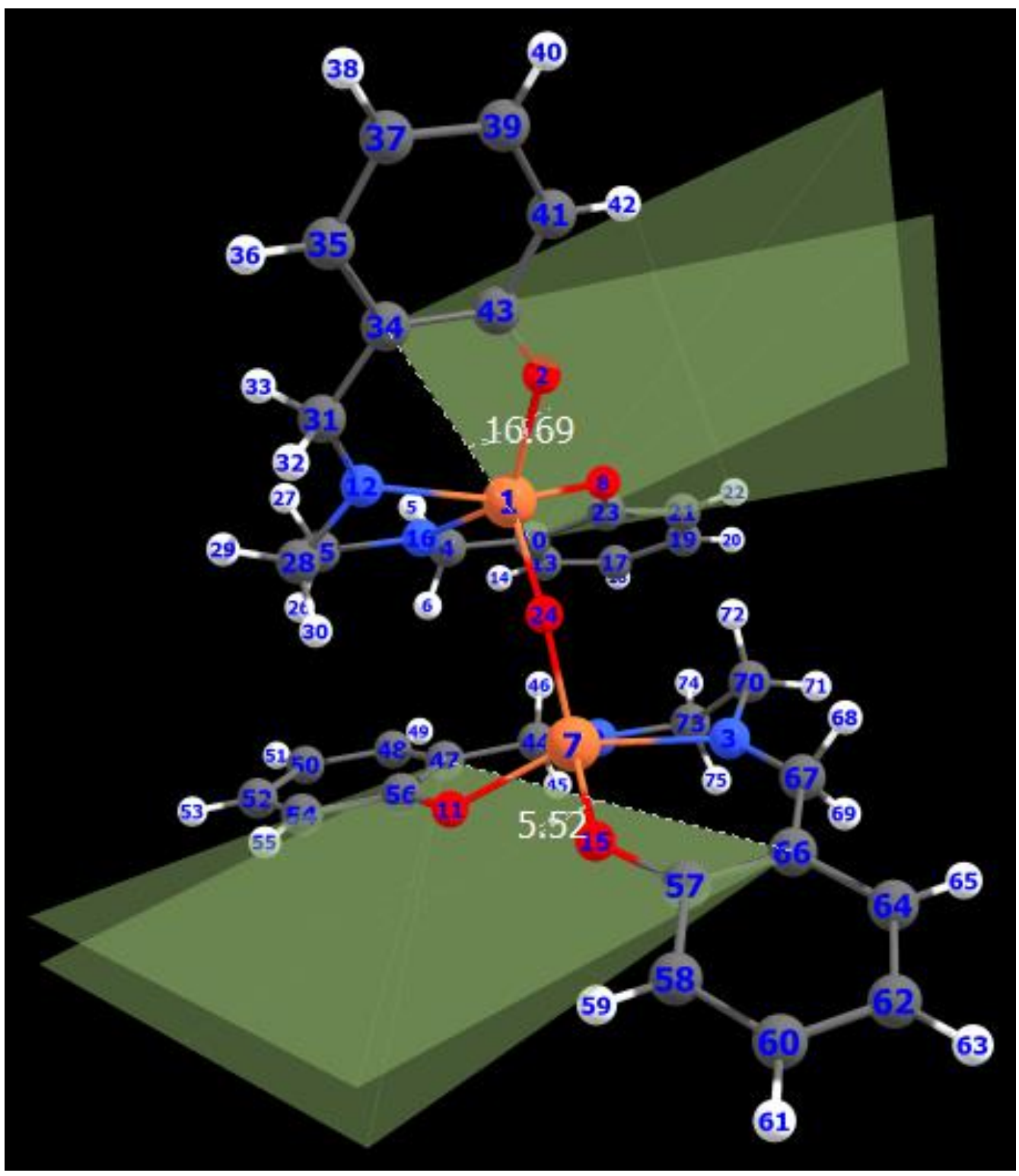


5 distortion (geometry from DFT optimisation). Angle measured C17-C6-C25-C34. Distortion $69^{\circ}$ :

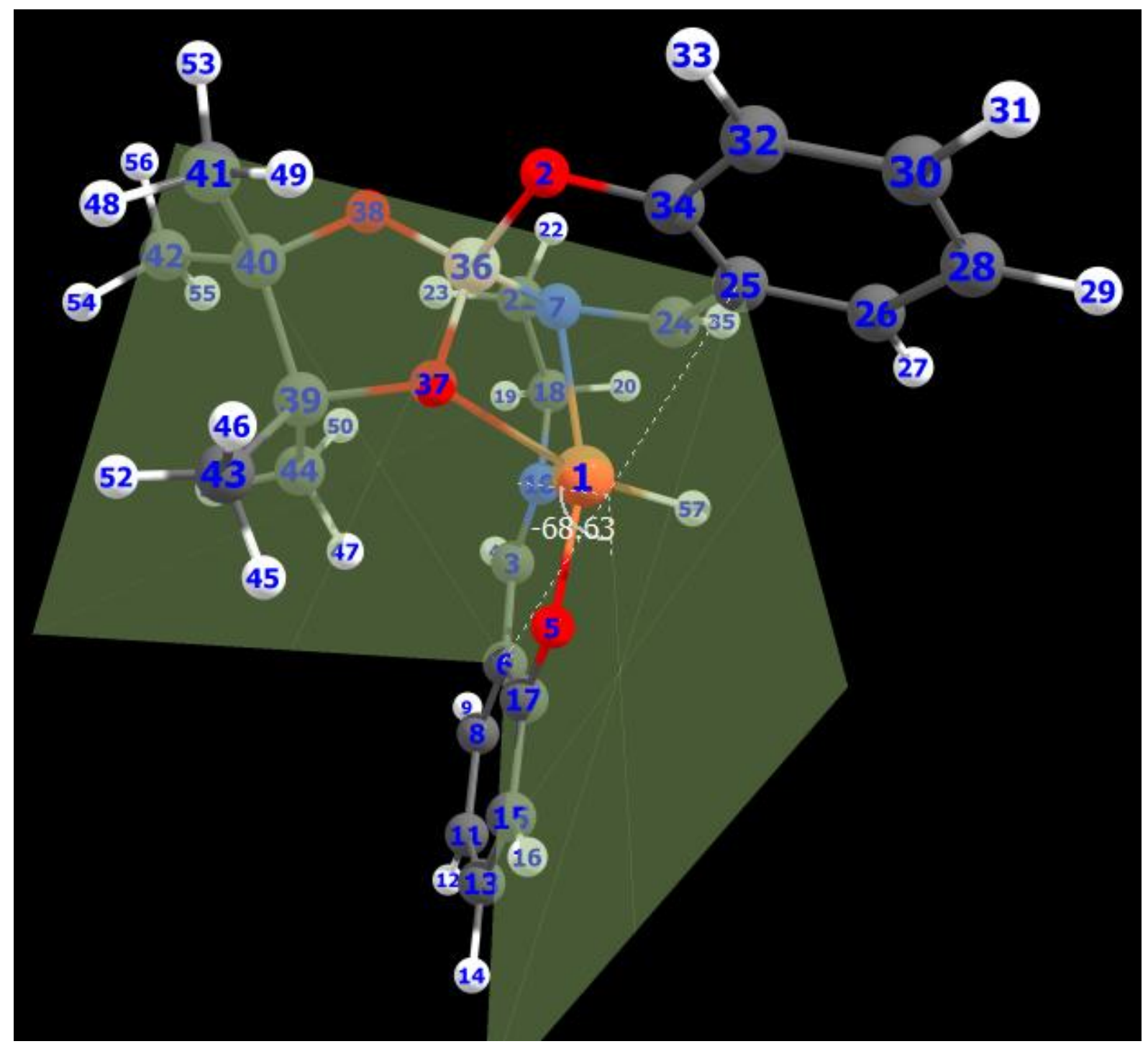


6 distortion (geometry from crystal structure). Angles measured C22-C23-C32-C35 and C15C12-C18-C19. Average distortion $6^{\circ}$ :

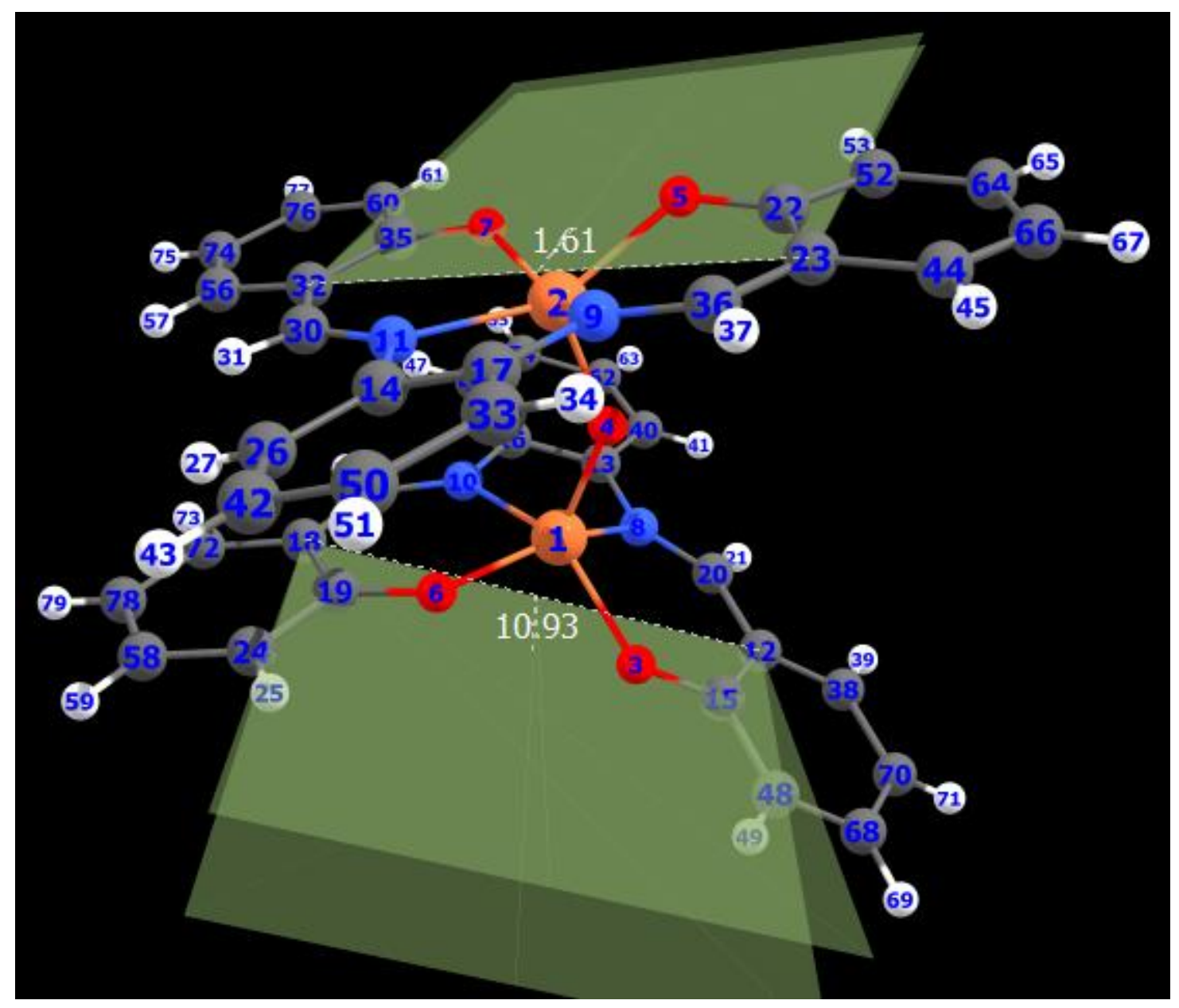




\section{Computational Data}

\section{Computational Parameters:}

Unrestricted density functional theory calculations were carried out using ORCA version 4.0.0.2, apart from nudged elastic band calculations, which were carried out using ORCA version 4.1.0. ${ }^{[13]}$ For all iron-containing species, all possible spin states were considered throughout; the relevant energies are compared below.

Geometry optimisations were performed using the BP functional making use of the resolution of the identity (RI) approximation. ${ }^{[14,15]}$ The zeroth order scalar relativistic approximation (ZORA) $)^{[16-18]}$ was used in conjunction with the relativistically recontracted ZORA-def2-TZVP(f) basis set and SARC/J auxiliary basis set on all atoms except carbon and hydrogen, for which the ZORA-def2-SVP and SARC/J basis sets were employed. ${ }^{[19-22]}$ The angular grid was Lebedev770 and the radial grid was set to 5.67; final grids were switched off. Atom-pairwise dispersion corrections with zero damping was used. ${ }^{[23]}$ Solvent effects were considered implicitly with the conductor-like polarizable continuum model, ${ }^{[24]}$ using acetonitrile as the modelled solvent, and explicitly by placing acetonitrile molecules on open coordination sites to test whether this resulted in a stabilisation. Where necessary, an inclusion of explicit solvent molecules is noted in the main text; the coordinates of the most stable complexes are reported below.

Frequency calculations were performed at the same level of theory as the geometry optimisations to afford thermodynamic corrections and identify any unwanted imaginary frequencies. The thermodynamic data reported in the main text and below are taken from these frequency calculations. 


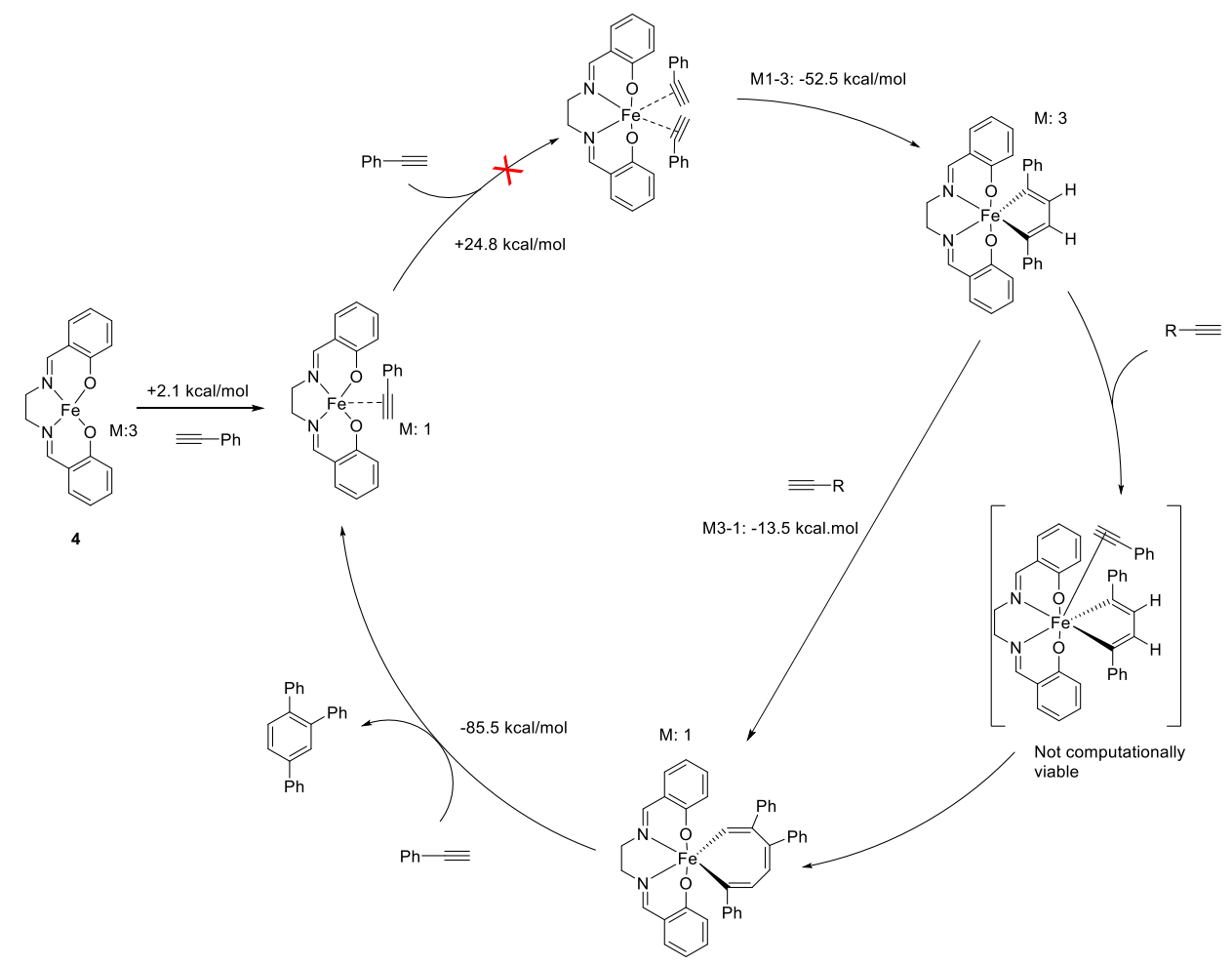

Scheme S1: Cycle starting from 4.

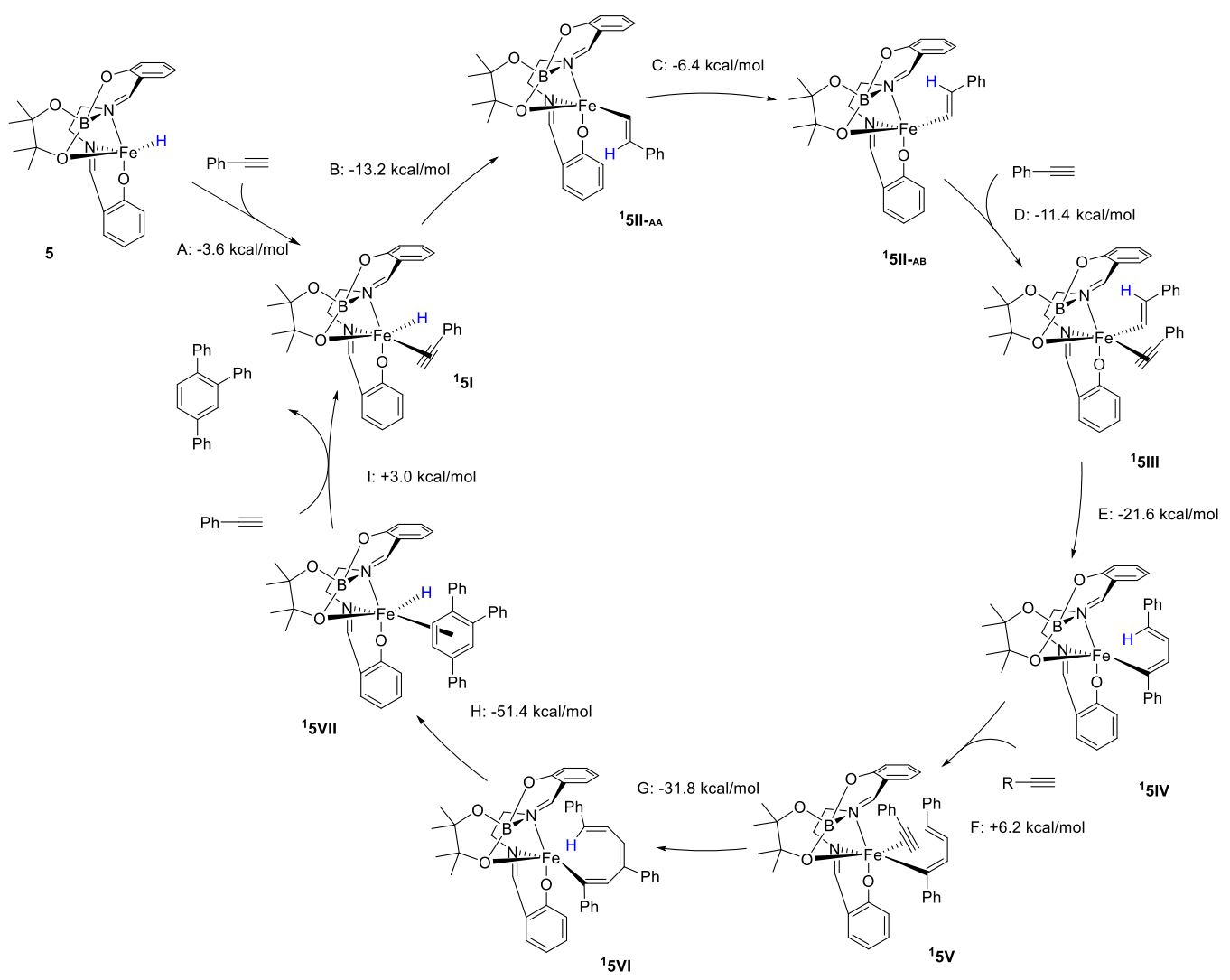

Scheme S2: Preliminary catalytic cycle. 


\section{Spin Multiplicity FSPE Comparison:}

NB: Spin multiplicity of selected intermediates investigated to understand lowest energy pathway. Data taken from geometry optimisations at level of theory specified in the computational parameters. Entries in red did not optimise to structures comparable to the closed shell species; mostly by the loss of coordination between various parts of the ligand and Fe.

\begin{tabular}{|c|c|c|c|}
\hline Species & FSPE $\left(E_{h}\right)$ & Mulliken Spin Pop. on Fe & $\Delta$ FSPE vs. closed shell ( $\mathrm{kcal} / \mathrm{mol})$ \\
\hline${ }^{15}$ & -2568.834448357149 & 0.000 & - \\
\hline${ }^{3} 5$ & -2568.824465322151 & 2.046 & +6.26 \\
\hline 55 & -2568.816987267153 & 3.558 & +10.96 \\
\hline${ }^{1} 5 \mathrm{I}-\mathrm{A}$ & -2877.255990868460 & 0.000 & - \\
\hline${ }^{3} 5 \mathrm{I}-\mathrm{A}$ & -2877.271907625301 & 2.015 & -9.99 \\
\hline $55 \mathrm{I}-\mathrm{A}$ & -2877.241736924726 & 3.450 & +8.94 \\
\hline${ }^{1} 5 \mathrm{II-AA}$ & -2877.282702738029 & 0.000 & - \\
\hline${ }^{3} 5 I I-A A$ & -2877.295409530525 & 2.039 & -7.97 \\
\hline 55 II-AA & -2877.305944360057 & 3.750 & -14.58 \\
\hline${ }^{1}$ 5III-AAA & -3185.708550583983 & 0.000 & - \\
\hline${ }^{3} 5$ III-AAA & -3185.740137718372 & 2.031 & -19.82 \\
\hline 5 5III-AAA & -3185.727336769411 & 3.425 & -11.79 \\
\hline${ }^{1} 5$ IV-AAA & -3185.791560585176 & 0.000 & - \\
\hline${ }^{3} 5$ IV-AAA & -3185.786568071152 & 1.990 & +3.13 \\
\hline 55IV-AAA & -3185.784953035167 & 3.524 & +4.15 \\
\hline${ }^{1} 5 \mathrm{~V}$-AAAA & -3494.204685741256 & 0.000 & - \\
\hline${ }^{3} 5 \mathrm{~V}$-AAAA & -3494.221719781923 & 2.044 & -19.56 \\
\hline $55 \mathrm{~V}$-AAAA & -3494.208249104686 & 3.441 & -11.11 \\
\hline${ }^{1}$ 5VI-AAAA & -3494.248077529709 & 0.000 & - \\
\hline${ }^{3} 5 \mathrm{VI}-\mathrm{AAAA}$ & -3494.246611408802 & 2.104 & +0.94 \\
\hline 55VI-AAAA & -3494.264185166238 & 3.717 & -10.11 \\
\hline${ }^{1}{ }_{5} \mathrm{VII}$ & -3494.313895957775 & 0.000 & - \\
\hline${ }^{3} 5 \mathrm{VII}$ & -3494.301254113329 & 2.017 & +7.96 \\
\hline${ }^{5} 5 \mathrm{VII}$ & -3494.299834436630 & 3.525 & +8.85 \\
\hline
\end{tabular}

Final single point energies $(\mathrm{kcal} / \mathrm{mol})$ of the triplet and quintet state of 5 relative to the singlet ground state computed with different density functionals at the geometry of ${ }^{1} \mathbf{5}$.

\begin{tabular}{|c|c|c|c|c|}
\hline Complex & BP86 & TPSSh & B3LYP & PBEO \\
\hline${ }^{1} 5$ & 0.00 & 0.00 & 0.00 & 0.00 \\
\hline${ }^{3} 5$ & 6.26 & 13.68 & 12.08 & 7.29 \\
\hline 55 & 10.96 & 22.43 & 15.92 & 6.41 \\
\hline
\end{tabular}




\section{Selected Bond Lengths:}

NB: Species for which crystallographic structural data is used are shown in red italics for comparison. If not specified, distance measures to nearest relevant atom. Averages used for dimers where appropriate. All distances given in $\AA$.

\begin{tabular}{|c|c|c|c|c|c|c|c|c|}
\hline Species & $\begin{array}{c}\mathrm{Fe}- \\
\mathrm{O}_{\text {salen1 }}\end{array}$ & $\begin{array}{c}\text { Fe- } \\
\mathrm{O}_{\text {salen2 }}\end{array}$ & $\begin{array}{c}\text { Fe- } \\
\mathrm{N}_{\text {salen1 }}\end{array}$ & $\begin{array}{c}\text { Fe- } \\
\mathrm{N}_{\text {salen2 }}\end{array}$ & $\mathrm{Fe}-\mathrm{H}$ & $\begin{array}{c}\mathrm{Fe}- \\
\mathrm{O}_{\text {Bpin }}\end{array}$ & $\begin{array}{l}\text { Fe- } \\
C_{\text {yne }}\end{array}$ & $\begin{array}{l}\text { Fe- } \\
C_{\text {ene }}\end{array}$ \\
\hline 1 & 1.92 & 1.95 & 2.12 & 2.11 & - & - & - & - \\
\hline $1-2 \mathrm{MeCN}$ & 1.95 & 1.96 & 1.91 & 1.92 & - & - & - & - \\
\hline 11 & 1.87 & 1.87 & 1.86 & 1.87 & 1.48 & - & - & - \\
\hline 111 & 1.90 & 1.91 & 1.89 & 1.90 & 1.68 & - & - & - \\
\hline 6 & 1.92 & 1.93 & 2.11 & 2.12 & - & - & - & - \\
\hline${ }^{3} \mathbf{4}$ & 1.89 & 1.89 & 1.88 & 1.88 & - & - & - & - \\
\hline${ }^{3} 4-\mathrm{MeCN}$ & 1.90 & 1.91 & 1.88 & 1.89 & - & - & - & - \\
\hline${ }^{1} 5$ & 1.83 & - & 1.83 & 2.06 & 1.48 & 2.51 & - & - \\
\hline${ }^{1} 51-\mathrm{A}$ & 1.90 & - & 1.93 & 2.02 & 1.46 & 2.37 & 2.10 & - \\
\hline${ }^{1} 51-\mathrm{B}$ & 1.90 & - & 1.92 & 2.02 & 1.47 & 2.26 & 2.10 & - \\
\hline TS $\left({ }^{1} 5 \mathrm{I}-\mathrm{A},{ }^{1} 5 \mathrm{II-AA}\right)$ & 1.93 & - & 1.96 & 2.04 & 1.50 & 2.16 & 1.99 & - \\
\hline TS( $\left.{ }^{1} 5 I-в,{ }^{1} 5 I I-в C\right)$ & 1.93 & - & 1.96 & 2.02 & 1.48 & 2.22 & 2.04 & - \\
\hline${ }^{1}$ 5II-AA & 1.87 & - & 1.94 & 1.97 & 3.15 & 1.99 & - & 1.95 \\
\hline${ }^{1} 5 I I-A B$ & 1.90 & - & 1.91 & 2.07 & 3.13 & 2.42 & - & 1.94 \\
\hline${ }^{1} 5 \mathrm{II-BC}$ & 1.85 & - & 1.83 & 1.99 & 3.20 & 2.12 & - & 1.97 \\
\hline${ }^{1}$ 5III-AAA & 1.92 & - & 2.00 & 2.07 & 3.06 & 2.18 & 2.04 & 1.99 \\
\hline${ }^{1} 5$ III-AAB & 1.90 & - & 2.00 & 2.14 & 3.08 & 2.20 & 1.99 & 1.98 \\
\hline${ }^{1} 5$ III-ABA & 1.90 & - & 1.92 & 2.04 & 3.33 & 2.26 & 2.16 & 1.94 \\
\hline${ }^{1} 5$ III-ABB & 1.85 & - & 1.82 & 2.00 & 3.29 & 2.14 & 3.43 & 1.92 \\
\hline TS $\left({ }^{1} 5\right.$ III-AAA, ${ }^{1} 5$ IV-AAA) & 1.91 & - & 1.99 & 2.09 & 3.18 & 2.24 & 2.04 & 2.01 \\
\hline TS $\left({ }^{1} 5\right.$ III-AAB, ${ }^{1} 5$ IV-AAB $)$ & 1.90 & - & 1.98 & 2.14 & 3.08 & 2.21 & 1.93 & 1.99 \\
\hline TS $\left({ }^{1} 5\right.$ III-ABA, $\left.{ }^{1} 5 I V-A B A\right)$ & 1.93 & - & 1.96 & 2.04 & 3.37 & 2.18 & 2.08 & 1.98 \\
\hline TS( $\left({ }^{1} 5 I I I-A B B,{ }^{1} 5 I V-A B B\right)$ & 1.92 & - & 1.97 & 2.04 & 3.34 & 2.22 & 1.96 & 1.97 \\
\hline${ }^{1}$ 5IV-AAA & 1.94 & - & 1.84 & 2.00 & 2.27 & 2.19 & - & 1.96 \\
\hline 15IV-AAB & 1.85 & - & 1.83 & 1.99 & 2.29 & 2.14 & - & 1.91 \\
\hline${ }^{1} 5$ IV-ABA & 1.92 & - & 1.98 & 2.01 & 2.19 & 2.05 & - & 1.99 \\
\hline${ }^{1} 5$ IV-ABB & 1.92 & - & 1.99 & 2.01 & 2.09 & 2.02 & - & 1.91 \\
\hline 15V-AAAA & 1.85 & - & 1.97 & 3.24 & 3.15 & 2.50 & 1.95 & 1.94 \\
\hline${ }^{15 V \text {-AABA }}$ & 1.87 & - & 1.92 & 2.06 & 3.11 & 2.32 & 2.16 & 1.94 \\
\hline${ }^{15 V-A B A A}$ & 1.91 & - & 2.01 & 2.07 & 3.35 & 2.27 & 2.05 & 2.11 \\
\hline${ }^{15 V-A B B A}$ & 1.91 & - & 2.02 & 2.08 & 3.29 & 2.25 & 2.03 & 1.99 \\
\hline${ }^{15}$ VII-AAAA & 1.85 & - & 1.86 & 2.07 & 2.93 & 2.23 & - & 1.98 \\
\hline${ }^{1} 5 \mathrm{VI}$-AAAB & 1.85 & - & 1.85 & 2.09 & 2.97 & 2.20 & - & 1.92 \\
\hline${ }^{1} 5 \mathrm{VI}$-АABA & 1.84 & - & 1.86 & 2.06 & 3.23 & 2.38 & - & 1.97 \\
\hline${ }^{1} 5 \mathrm{VI}$-AABB & 1.84 & - & 1.86 & 2.10 & 3.24 & 2.30 & - & 1.91 \\
\hline${ }^{1}$ 5VI-ABAA & 1.87 & - & 2.00 & 2.13 & 2.84 & 2.08 & - & 2.00 \\
\hline${ }^{1} 5 \mathrm{VI}$-АBAB & 1.85 & - & 1.97 & 2.15 & 2.87 & 2.09 & - & 1.90 \\
\hline${ }^{1} 5 \mathrm{VI}$-ABBA & 1.87 & - & 2.00 & 2.12 & 2.69 & 2.14 & - & 1.99 \\
\hline${ }^{1} 5 \mathrm{VI}$-ABBB & 1.87 & - & 1.97 & 2.00 & 2.82 & 2.03 & - & 1.91 \\
\hline${ }^{1} 5 \mathrm{VII}$ & 1.82 & 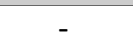 & 1.84 & 2.06 & 1.48 & 2.47 & - & 3.29 \\
\hline
\end{tabular}




\section{Selected Angles:}

NB: Species for which crystallographic structural data is used are shown in red italics for comparison; distortion refers to the dihedral angle specified in section 7.

\begin{tabular}{|c|c|c|c|}
\hline Species & Distortion & $\mathbf{N}_{\text {salen }}-\mathrm{Fe}-\mathrm{N}_{\text {salen }}$ & $\mathbf{O}_{\text {salen }}-\mathrm{Fe}-\mathbf{O}_{\text {salen/Bpir }}$ \\
\hline 1 & $11^{\circ}$ & $76^{\circ}$ & $93^{\circ}$ \\
\hline $1-2 \mathrm{MeCN}$ & $1^{\circ}$ & $86^{\circ}$ & $85^{\circ}$ \\
\hline 11 & $0^{\circ}$ & $86^{\circ}$ & $85^{\circ}$ \\
\hline 1II & $2^{\circ}$ & $85^{\circ}$ & $84^{\circ}$ \\
\hline 6 & $6^{\circ}$ & $91^{\circ}$ & $77^{\circ}$ \\
\hline${ }^{3} 4$ & $4^{\circ}$ & $86^{\circ}$ & $86^{\circ}$ \\
\hline${ }^{3} 4-\mathrm{MeCN}$ & $0^{\circ}$ & $85^{\circ}$ & $85^{\circ}$ \\
\hline${ }^{15}$ & $69^{\circ}$ & $82^{\circ}$ & $91^{\circ}$ \\
\hline${ }^{1} 51-A$ & $61^{\circ}$ & $82^{\circ}$ & $91^{\circ}$ \\
\hline${ }^{1} 51-$ в & $60^{\circ}$ & $82^{\circ}$ & $91^{\circ}$ \\
\hline TS $\left({ }^{1} 5\right.$ I-A, ${ }^{1} 5$ II-AA $)$ & $53^{\circ}$ & $82^{\circ}$ & $87^{\circ}$ \\
\hline $\mathrm{TS}\left({ }^{1} 5 \mathrm{I-в},{ }^{1} 5 \mathrm{II-вC}\right)$ & $59^{\circ}$ & $82^{\circ}$ & $87^{\circ}$ \\
\hline${ }^{1} 5 I I-A A$ & $50^{\circ}$ & $84^{\circ}$ & $97^{\circ}$ \\
\hline${ }^{1} 5 I I-A B$ & $60^{\circ}$ & $82^{\circ}$ & $91^{\circ}$ \\
\hline${ }^{15}$ III-BC & $57^{\circ}$ & $86^{\circ}$ & $87^{\circ}$ \\
\hline${ }^{1}$ 5III-AAA & $61^{\circ}$ & $80^{\circ}$ & $89^{\circ}$ \\
\hline${ }^{15}$ IIII-AAB & $60^{\circ}$ & $80^{\circ}$ & $90^{\circ}$ \\
\hline${ }^{1}$ 5III-ABA & $57^{\circ}$ & $82^{\circ}$ & $87^{\circ}$ \\
\hline${ }^{15} 5 I I I-A B B$ & $60^{\circ}$ & $86^{\circ}$ & $88^{\circ}$ \\
\hline TS( ${ }^{1} 5$ III-AAA, ${ }^{1} 5$ IV-AAA $)$ & $58^{\circ}$ & $80^{\circ}$ & $87^{\circ}$ \\
\hline TS $\left({ }^{1} 5\right.$ III-AAB, $\left.{ }^{1} 5 I V-А A B\right)$ & $58^{\circ}$ & $80^{\circ}$ & $90^{\circ}$ \\
\hline TS $\left({ }^{1} 5\right.$ III-ABA, ${ }^{1} 5$ IV-ABA $)$ & $52^{\circ}$ & $81^{\circ}$ & $85^{\circ}$ \\
\hline 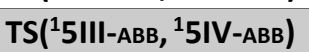 & $54^{\circ}$ & $82^{\circ}$ & $85^{\circ}$ \\
\hline${ }^{1} 5$ IV-AAA & $57^{\circ}$ & $85^{\circ}$ & $87^{\circ}$ \\
\hline${ }^{15} 5$ IV-AAB & $53^{\circ}$ & $86^{\circ}$ & $87^{\circ}$ \\
\hline${ }^{15}$ IIV-ABA & $58^{\circ}$ & $81^{\circ}$ & $90^{\circ}$ \\
\hline${ }^{1} 5$ IV-ABB & $51^{\circ}$ & $82^{\circ}$ & $90^{\circ}$ \\
\hline${ }^{15 V}$-AAAA & $41^{\circ}$ & $68^{\circ}$ & $93^{\circ}$ \\
\hline${ }^{1} 5 \mathrm{~V}$-AABA & $58^{\circ}$ & $81^{\circ}$ & $89^{\circ}$ \\
\hline${ }^{1} 5 \mathrm{~V}$-ABAA & $72^{\circ}$ & $79^{\circ}$ & $89^{\circ}$ \\
\hline${ }^{1} 5 \mathrm{~V}$-ABBA & $68^{\circ}$ & $79^{\circ}$ & $88^{\circ}$ \\
\hline${ }^{1}$ 5VI-AAAA & $51^{\circ}$ & $83^{\circ}$ & $85^{\circ}$ \\
\hline${ }^{1} 5 \mathrm{VI}$-AAAB & $49^{\circ}$ & $84^{\circ}$ & $86^{\circ}$ \\
\hline${ }^{1}$ 5VI-AABA & $63^{\circ}$ & $83^{\circ}$ & $89^{\circ}$ \\
\hline${ }^{1} 5 \mathrm{VI}$-AABB & $60^{\circ}$ & $83^{\circ}$ & $88^{\circ}$ \\
\hline${ }^{1}$ 5VI-ABAA & $51^{\circ}$ & $80^{\circ}$ & $94^{\circ}$ \\
\hline${ }^{1} 5 \mathrm{VI}$-ABAB & $47^{\circ}$ & $81^{\circ}$ & $95^{\circ}$ \\
\hline${ }^{1} 5 \mathrm{VI}$-ABBA & $56^{\circ}$ & $79^{\circ}$ & $93^{\circ}$ \\
\hline${ }^{1}$ 5VI-ABBB & $51^{\circ}$ & $82^{\circ}$ & $92^{\circ}$ \\
\hline${ }^{1} 5 \mathrm{VII}$ & $62^{\circ}$ & $86^{\circ}$ & $94^{\circ}$ \\
\hline
\end{tabular}




\section{Electronic and Energetic Data:}

NB: FSPE taken from geometry optimisation calculation; thermodynamic data are obtained from frequency calculations with $\mathrm{T}=298.15 \mathrm{~K}$.

\begin{tabular}{|c|c|c|c|c|c|}
\hline Species & $\begin{array}{l}\text { Mulliken } \\
\text { spin pop. } \\
\text { on Fe }\end{array}$ & FSPE $\left(E_{h}\right)$ & $\mathrm{H}\left(E_{\mathrm{h}}\right)$ & $\mathrm{TS}\left(E_{\mathrm{h}}\right)$ & $\mathbf{G}\left(E_{\mathrm{h}}\right)$ \\
\hline $1-2 \mathrm{MeCN}$ & $0.720,0.771$ & -4654.43748640570 & -4653.71922539 & -0.11659619 & -4653.83582158 \\
\hline 11 & 0.984 & -2157.25477458926 & -2156.94195869 & -0.06050137 & -2157.00246007 \\
\hline 1II & $0.299,1.847$ & -4314.53442020515 & -4313.90890012 & -0.09673801 & -4314.00563814 \\
\hline${ }^{3} 4$ & 2.135 & -2156.66923838669 & -2156.36473784 & -0.06086822 & -2156.42560606 \\
\hline${ }^{3} 4-\mathrm{MeCN}$ & 2.067 & -2289.52106738926 & -2289.15957478 & -0.06937084 & -2289.22894562 \\
\hline${ }^{15}$ & 0.000 & -2568.83444835714 & -2568.32840485 & -0.07688586 & -2568.40529071 \\
\hline IA & 0.000 & -2877.25599086846 & -2876.63451887 & -0.09187966 & -2876.72639853 \\
\hline ІB & 0.000 & -2877.25863834026 & -2876.63786883 & -0.09106566 & -2876.72893449 \\
\hline$T S\left(I_{A}, I_{A A}\right)$ & 0.000 & -2877.25064095235 & -2876.63159519 & -0.09112190 & -2876.72271709 \\
\hline $\mathrm{TS}(\mathrm{IB}, \mathrm{IlBC})$ & 0.000 & -2877.25350659287 & -2876.63491733 & -0.09011388 & -2876.72503121 \\
\hline IIAA & 0.000 & -2877.28270273802 & -2876.65539790 & -0.09199502 & -2876.74739292 \\
\hline$\|_{A B}$ & 0.000 & -2877.30692680372 & -2876.66396769 & -0.09356629 & -2876.75753398 \\
\hline IIBC & 0.000 & -2877.30734091867 & -2876.68334880 & -0.09027057 & -2876.77361937 \\
\hline IllaAA & 0.000 & -3185.70855058398 & -3184.96235307 & -0.10574164 & -3185.06809471 \\
\hline IIIAAB & 0.000 & -3185.70642702484 & -3184.96154556 & -0.10596768 & -3185.06751324 \\
\hline IIIIABA & 0.000 & -3185.72297476042 & -3184.97985919 & -0.10585526 & -3185.08571446 \\
\hline IIIABB & 0.000 & -3185.72751687981 & -3184.98434041 & -0.10674241 & -3185.09108282 \\
\hline TS(IIIAAA, IV IAAA) & 0.000 & -3185.70577246185 & -3184.96549149 & -0.10577379 & -3185.07126528 \\
\hline TS(IIIAAB, IV & 0.000 & -3185.704523375644 & -3184.96241730 & -0.10559160 & -3185.06800890 \\
\hline$T S\left(I I_{A B A}, I V_{A B A}\right)$ & 0.000 & -3185.708855694618 & -3184.96923976 & -0.10433921 & -3185.07357897 \\
\hline$T S\left(I I I_{A B B}, I V_{A B B}\right)$ & 0.000 & -3185.704122197608 & -3184.96297693 & -0.10455459 & -3185.06753152 \\
\hline IVAAA & 0.000 & -3185.791560585176 & -3185.04739208 & -0.10402038 & -3185.15141246 \\
\hline IVAAB & 0.000 & -3185.787808673922 & -3185.04234318 & -0.10406633 & -3185.14640952 \\
\hline IV & 0.000 & -3185.771915133326 & -3185.02887084 & -0.10235482 & -3185.13122566 \\
\hline IV $V_{A B B}$ & 0.000 & -3185.766826933595 & -3185.02201092 & -0.10349442 & -3185.12550533 \\
\hline VAAAA & 0.000 & -3494.204685741256 & -3493.34083765 & -0.11879123 & -3493.45962889 \\
\hline$V_{\text {AABA }}$ & 0.000 & -3494.192189760136 & -3493.32657195 & -0.11874483 & -3493.44531678 \\
\hline VabAa & 0.000 & -3494.154094632398 & -3493.28927631 & -0.11871198 & -3493.40798829 \\
\hline$V_{\text {ABBA }}$ & 0.000 & -3494.178775690738 & -3493.31180063 & -0.11922333 & -3493.43102396 \\
\hline VIaAaA & 0.000 & -3494.248077529709 & -3493.38336775 & -0.11611320 & -3493.49948095 \\
\hline VIaAAB & 0.000 & -3494.249561733670 & -3493.38234363 & -0.11655871 & -3493.49890234 \\
\hline VlaAba & 0.000 & -3494.248387781627 & -3493.38264684 & -0.11674014 & -3493.49938698 \\
\hline VIAABb & 0.000 & -3494.249672829259 & -3493.38197698 & -0.11627930 & -3493.49825627 \\
\hline VlabaA & 0.000 & -3494.232155967473 & -3493.36828345 & -0.11604747 & -3493.48433092 \\
\hline VIABAB & 0.000 & -3494.242687013314 & -3493.37697933 & -0.11683320 & -3493.49381253 \\
\hline VIABBA & 0.000 & -3494.231800152516 & -3493.36647549 & -0.11540719 & -3493.48188268 \\
\hline VlabBB & 0.000 & -3494.240749252792 & -3493.37526244 & -0.11623471 & -3493.49149715 \\
\hline VII & 0.000 & -3494.313934278949 & -3493.44775032 & -0.11611260 & -3493.56386292 \\
\hline
\end{tabular}




\section{Reaction Step Energy Changes for Catalytic Cycle:}

NB: All energies are given as changes with respect to the previous step in $\mathrm{kcal} / \mathrm{mol}$. Data for steps computed with BP86 electronic energies and thermodynamic corrections (BP86/BP86) and for those computed with PBE0 electronic energies and BP86 thermodynamic corrections (BP86/PBE0) are given.

\begin{tabular}{|c|c|c|c|c|c|c|c|}
\hline \multirow[b]{2}{*}{ AAAA path } & \multicolumn{4}{|c|}{ BP86/BP86 } & \multicolumn{3}{|c|}{ BP86/PBE0 } \\
\hline & Step & $\Delta \mathrm{H}$ & $\mathrm{T} \Delta \mathrm{S}$ & $\Delta G$ & $\Delta \mathrm{H}$ & $\mathrm{T} \Delta \mathrm{S}$ & $\Delta G$ \\
\hline${ }^{1} 5 \rightarrow I_{A}$ & $A$ & -17.33 & +13.78 & -3.55 & -12.14 & +18.97 & +1.64 \\
\hline$I_{A} \rightarrow T S\left(I_{A}, I_{A A}\right)$ & TS-B & +1.83 & +0.48 & +2.31 & & & \\
\hline$I_{A} \rightarrow I_{A A}$ & B & -13.10 & -0.07 & -13.17 & -4.33 & +8.70 & -4.40 \\
\hline $\mathrm{IIAA}_{\mathrm{A}} \rightarrow \mathrm{III}_{\mathrm{AAA}}$ & $\mathrm{C}$ & -17.86 & +14.56 & -3.30 & -6.74 & +25.69 & +7.82 \\
\hline IIIAAA $_{\text {A }} \rightarrow$ TS(III AAA, IV & TS-D & -1.97 & -0.02 & -1.99 & & & \\
\hline $\mathrm{III}_{\mathrm{AAA}} \rightarrow \mathrm{IV}_{\mathrm{AAA}}$ & D & -53.36 & +1.08 & -52.28 & -60.14 & -5.69 & -59.06 \\
\hline IV $V_{A A A} \rightarrow V_{A A A A}$ & $\mathrm{E}$ & -9.38 & +13.92 & +4.54 & -5.75 & +17.56 & +8.17 \\
\hline $\mathrm{V}_{\mathrm{AAAA}} \rightarrow \mathrm{VI}_{\mathrm{AAAA}}$ & $\mathrm{F}$ & -26.69 & +1.68 & -25.01 & -28.31 & +0.06 & -26.63 \\
\hline $\mathrm{VI}_{\mathrm{AAAA}} \rightarrow \mathrm{VII}$ & G & -40.40 & 0.00 & -40.40 & -47.30 & -6.90 & -47.30 \\
\hline $\mathrm{VII} \rightarrow \mathrm{IA}_{\mathrm{A}}$ & $\mathrm{H}$ & +4.41 & -1.40 & +3.01 & 2.84 & -2.97 & +1.44 \\
\hline
\end{tabular}

\begin{tabular}{|c|c|c|c|c|c|c|c|}
\hline & \multicolumn{4}{|c|}{ BP86/BP86 } & \multicolumn{3}{|c|}{ BP86/PBE0 } \\
\hline AABA path & Step & $\Delta \mathrm{H}$ & $\mathrm{T} \Delta \mathrm{S}$ & $\Delta \mathrm{G}$ & $\Delta \mathrm{H}$ & $\mathrm{T} \Delta \mathrm{S}$ & $\Delta \mathrm{G}$ \\
\hline${ }^{1} 5 \rightarrow I_{A}$ & $A$ & -17.33 & +13.78 & -3.55 & -12.14 & +18.97 & +1.64 \\
\hline$I_{A} \rightarrow T S\left(I_{A}, I_{A A}\right)$ & TS-B & +1.83 & +0.48 & +2.31 & & & \\
\hline$I_{A} \rightarrow I_{A A}$ & B & -13.10 & -0.07 & -13.17 & -4.33 & +8.70 & -4.40 \\
\hline$\left\|_{A A} \rightarrow\right\| \|_{A A B}$ & C & -17.35 & +14.42 & -2.93 & -6.63 & +25.15 & +7.79 \\
\hline $\mathrm{III}_{\mathrm{AAB}} \rightarrow \mathrm{TS}\left(\mathrm{II} \mathrm{I}_{\mathrm{A}} \mathrm{B}, \mathrm{IV}\right.$ AAB $)$ & TS-D & -0.55 & +0.24 & -0.31 & & & \\
\hline\|\|$_{A A B} \rightarrow I_{A A B}$ & D & -50.70 & +1.19 & -49.51 & -59.40 & -7.51 & -58.21 \\
\hline $\mathrm{IV}_{\mathrm{AAB}} \rightarrow \mathrm{V}_{\mathrm{AABA}}$ & $\mathrm{E}$ & -3.60 & +13.98 & +10.38 & +3.02 & +20.60 & +17.00 \\
\hline $\mathrm{V}_{\mathrm{AABA}} \rightarrow \mathrm{V}_{\mathrm{AABA}}$ & $\mathrm{F}$ & -35.19 & +1.26 & -33.93 & -38.09 & -1.64 & -36.83 \\
\hline $\mathrm{VIIABA}_{\mathrm{A}} \rightarrow \mathrm{VII}$ & G & -40.85 & +0.39 & -40.46 & -47.14 & -5.90 & -46.75 \\
\hline $\mathrm{VII} \rightarrow \mathrm{I}_{\mathrm{A}}$ & $\mathrm{H}$ & +4.41 & -1.40 & +3.01 & +2.84 & -2.97 & +1.44 \\
\hline
\end{tabular}


Catalytic cycle via $\mathrm{V}_{\mathrm{ABB}}$ with $3 \mathrm{D}$ representations of intermediates

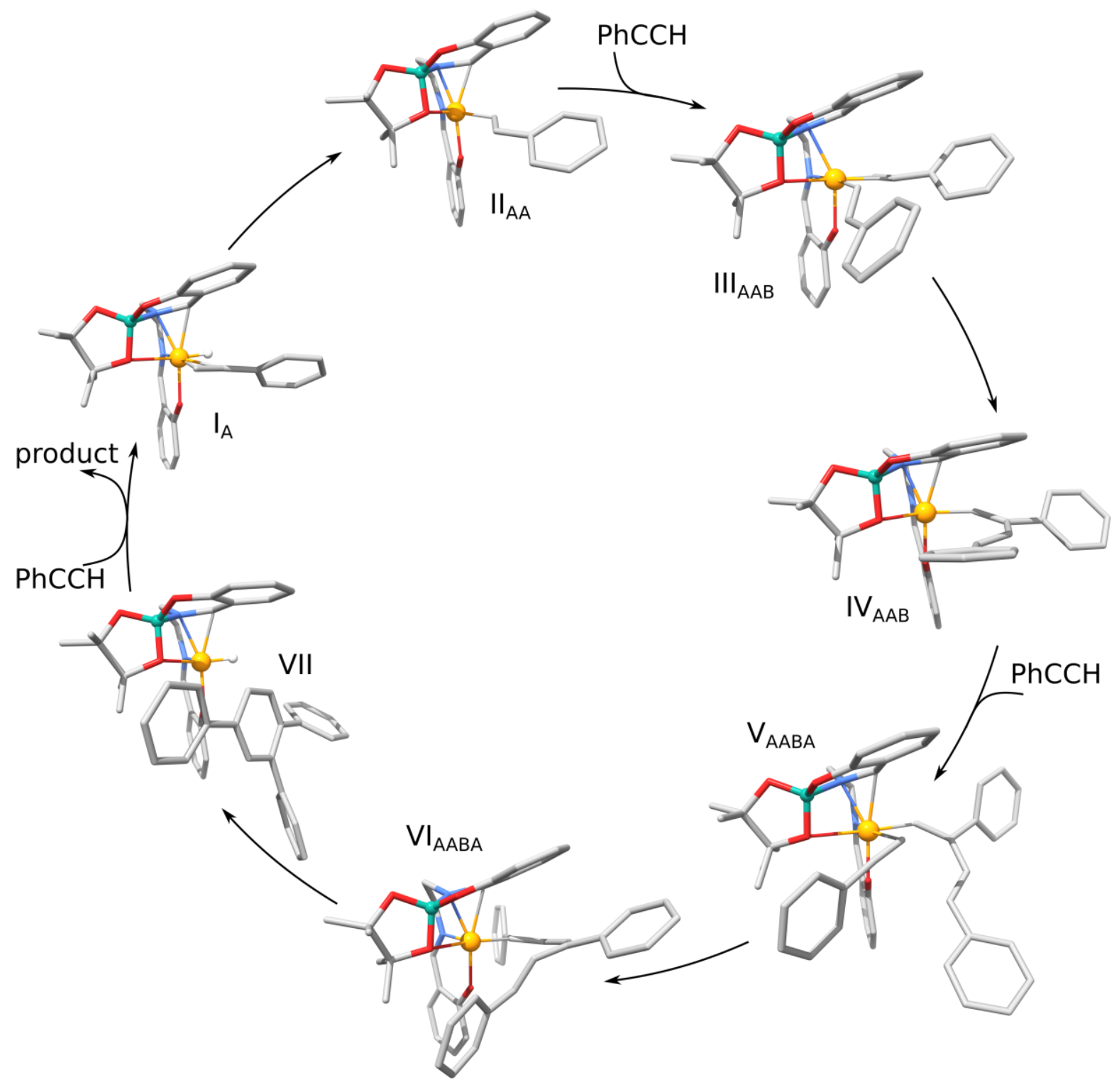


Catalytic cycle via $\mathrm{V}_{\text {AAAA }}$ with 3D representations of intermediates:

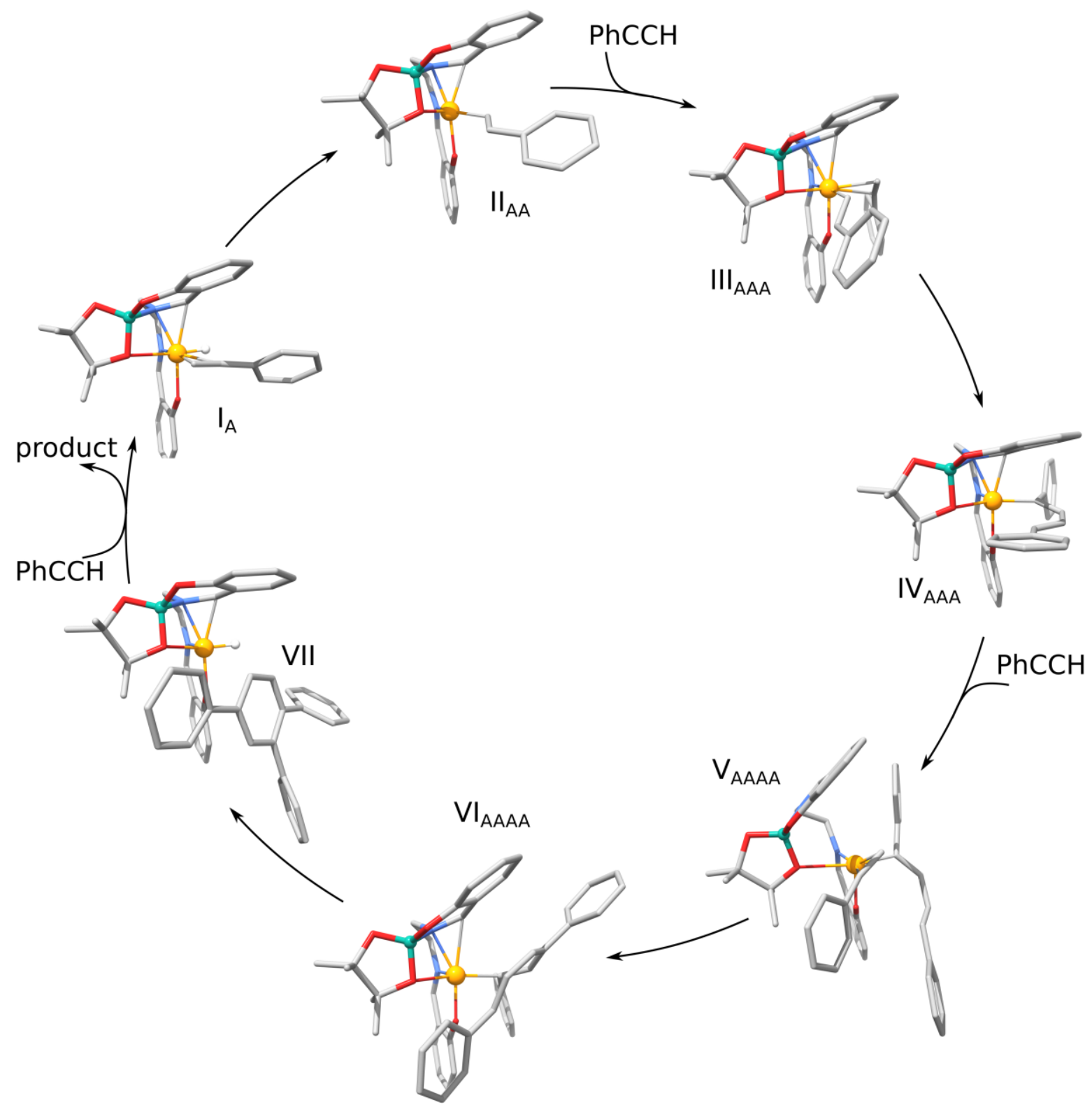




\section{Spectra}

\section{Stoichiometric Reaction of 1 and HBpin}

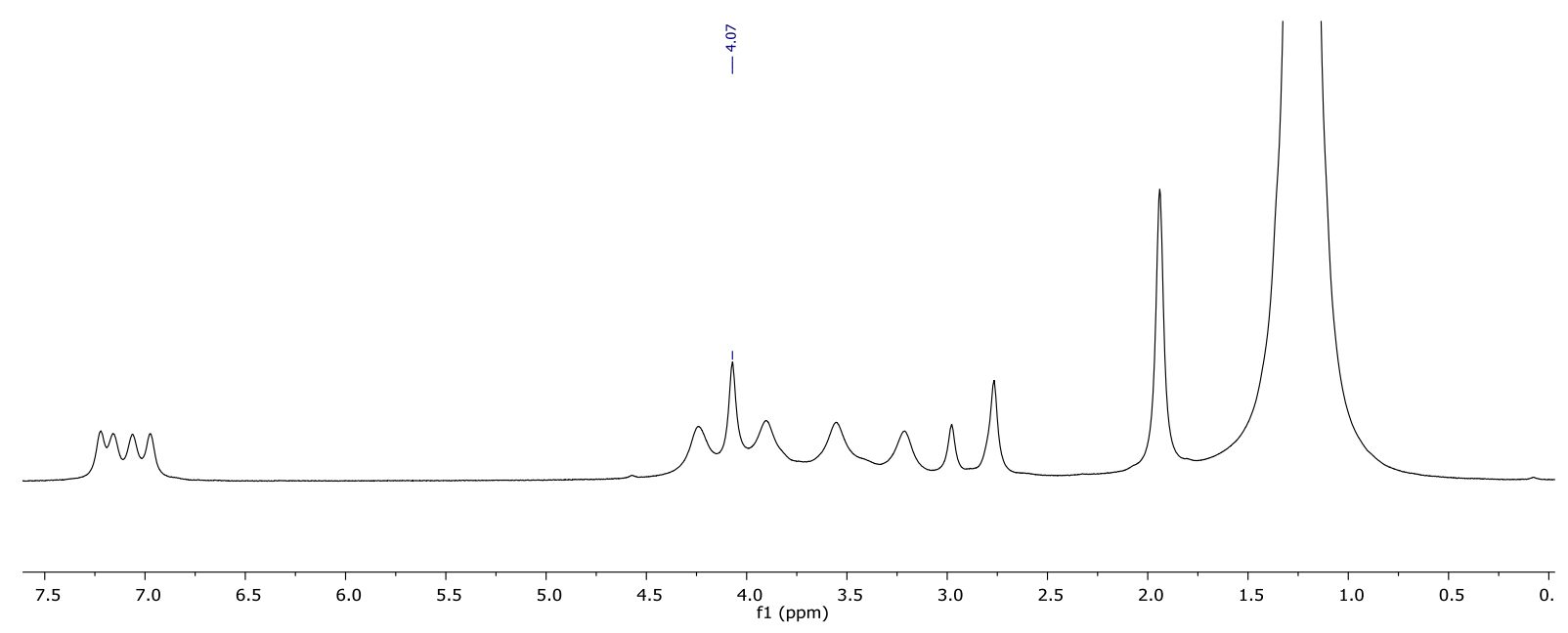

Figure S19: ${ }^{1} \mathrm{H}$ NMR of 1 and HBpin reaction. Singlet at $4.07 \mathrm{ppm}$ evidence for $\mathrm{H}_{2}$.

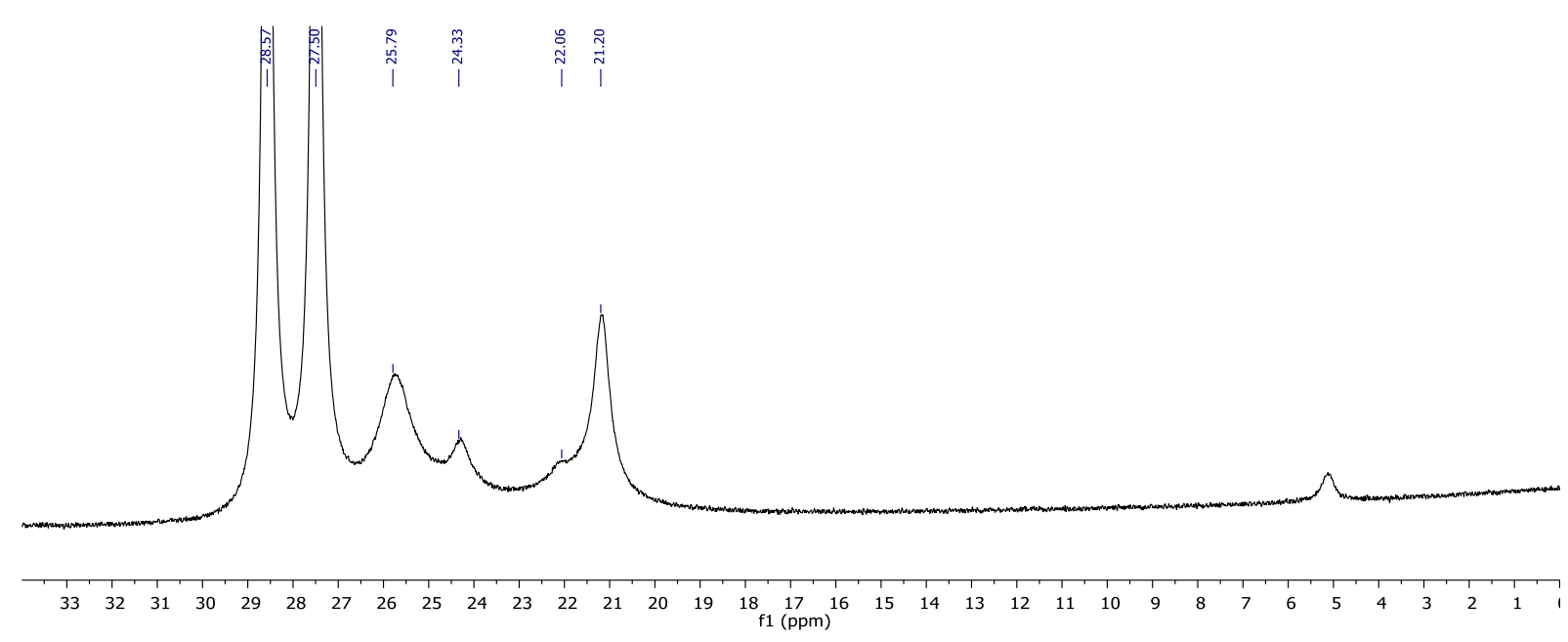

Figure S20: ${ }^{11} \mathrm{~B}$ NMR of $1+\mathrm{HBpin}$ reaction. Large doublet at $28 \mathrm{ppm}$ : HBpin, singlet at 21.2 ppm: hydrolysed HBpin. Peaks at 25.8, 24.3 and $22.1 \mathrm{ppm}$ are new, evidence for by-products of the reduction of $[\mathrm{Fe}(\text { salen })]_{2}-\mu$-oxo or the formation of B-adducts with the complex. 
Stoichiometric reaction of 1 with 20 equivalents of HBpin/DBpin

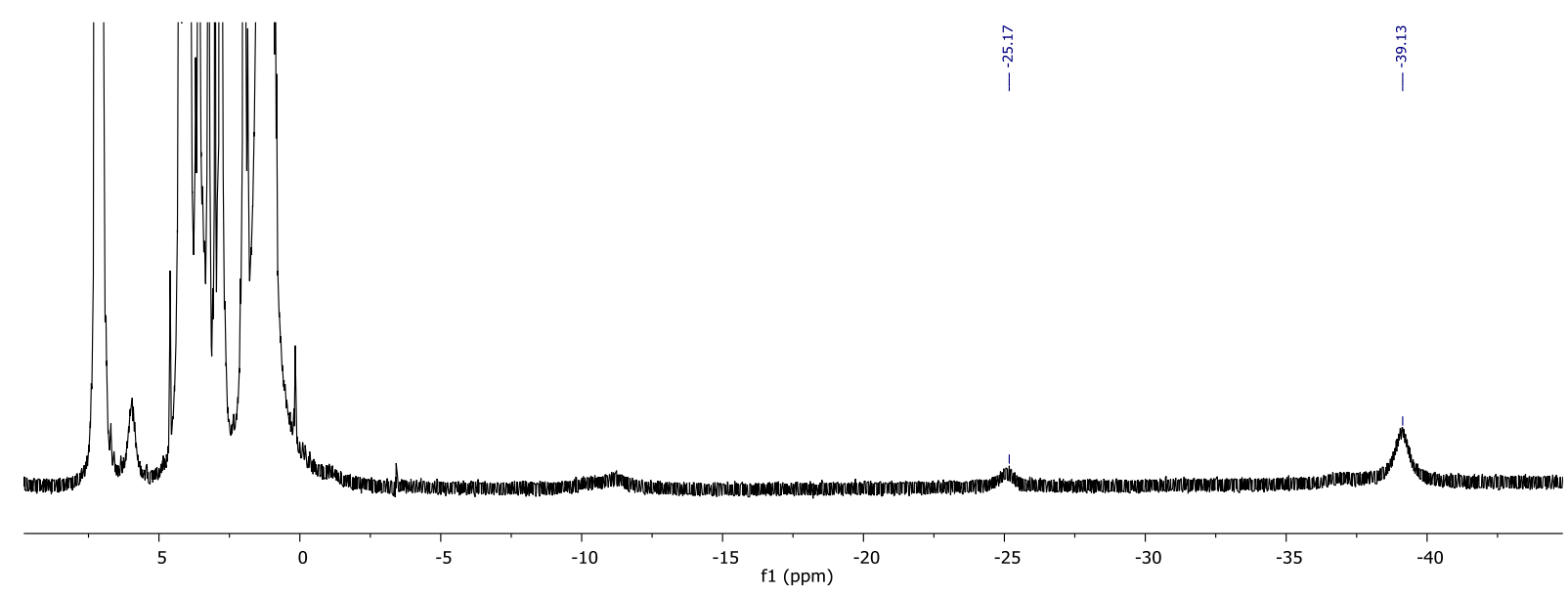

Figure S21: ${ }^{1} \mathrm{H}$ NMR focused on hydride region of reaction of 1 and 20 eq. $\mathrm{HBpin}$ in $\mathrm{CD}_{3} \mathrm{CN}$.

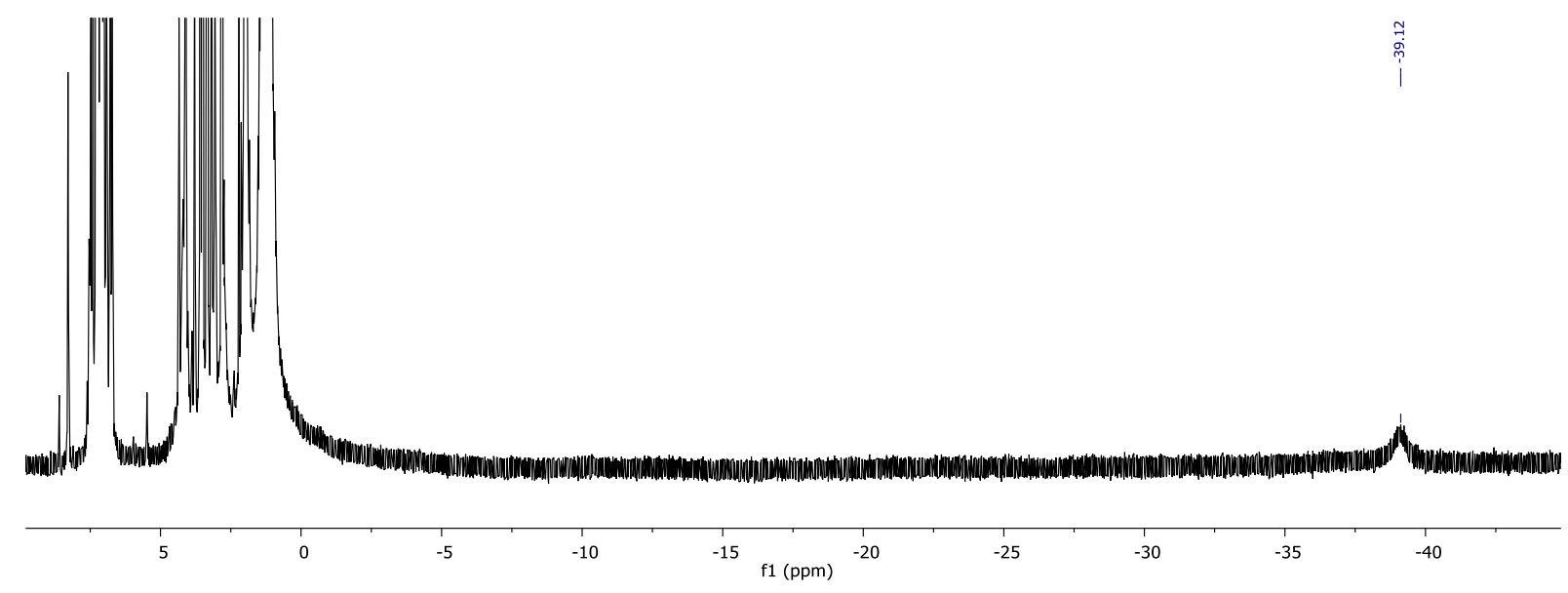

Figure S22: ${ }^{1} \mathrm{H}$ NMR focused on hydride region of stoichiometric reaction of 1 and 20 eq. DBpin in $\mathrm{CD}_{3} \mathrm{CN}$. 
Paramagnetic spectra of 1 becoming diamagnetic on addition of HBpin

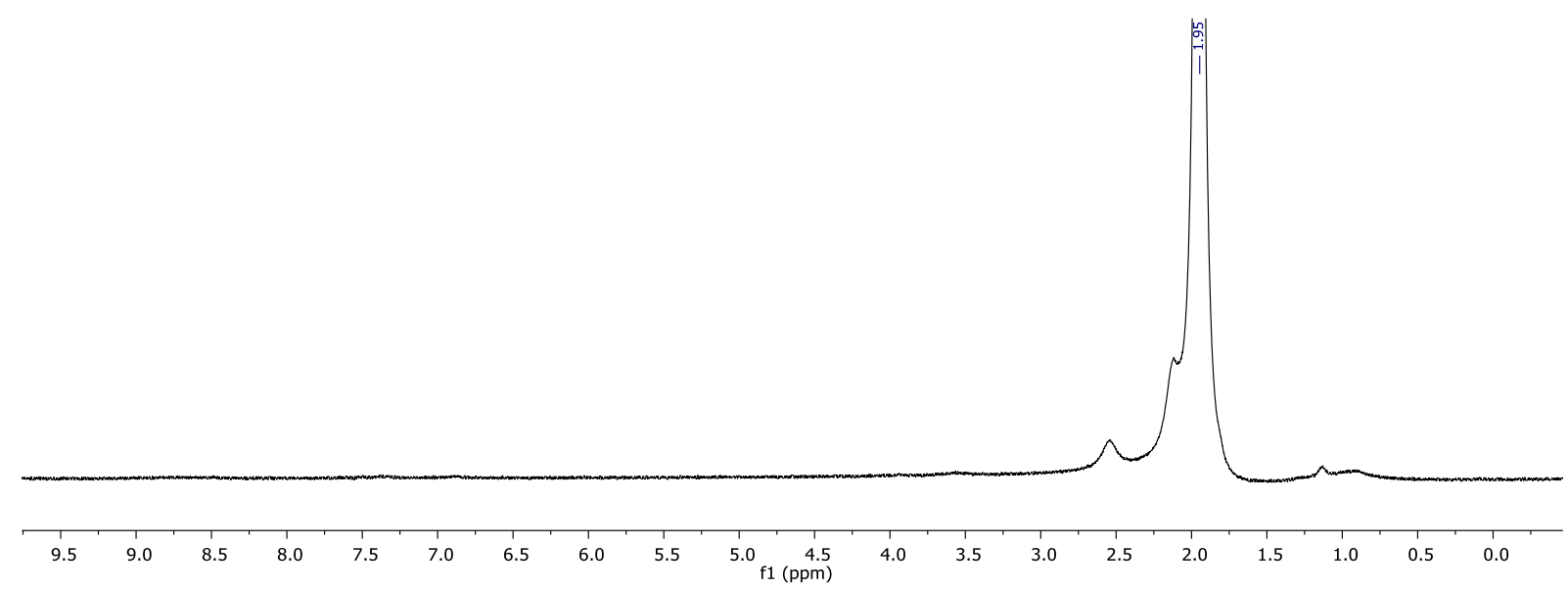

Figure S23: ${ }^{1} \mathrm{H}$ NMR of 1 in $\mathrm{CD}_{3} \mathrm{CN}$, focused on diamagnetic region. Note absence of signals (peak at $1.95 \mathrm{ppm}$ is residual protic $\mathrm{MeCN}$ in NMR solvent).

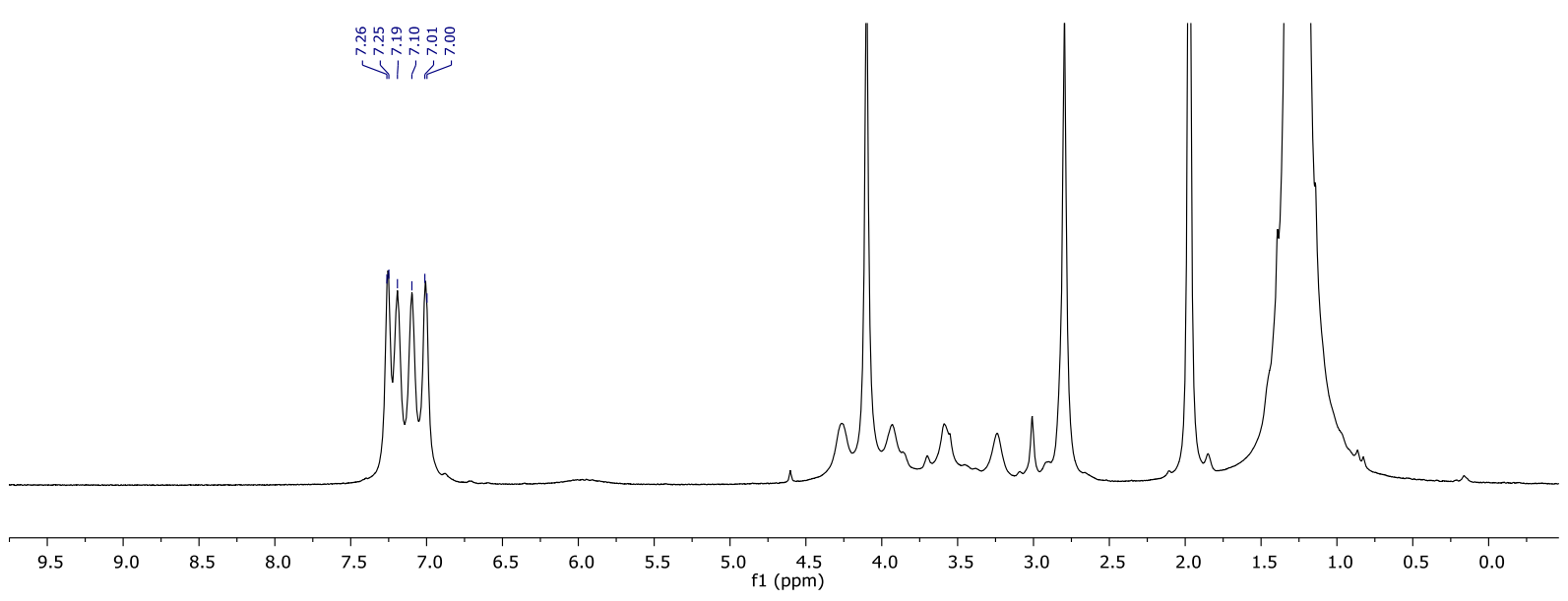

Figure S24: ${ }^{1} \mathrm{H}$ NMR of complex 1 with 20 equivalents of $\mathrm{HBpin}$ after 4 days at $\mathrm{RT}$ in $\mathrm{CD}_{3} \mathrm{CN}$, focused on diamagnetic region. Note new signals appearing in aromatic region 7.26-7.00 $\mathrm{ppm}$, indicating presence of aromatic protons residing on a diamagnetic species. 


\section{Spectroscopic Characterisation of Products}

3a ( $\left.{ }^{1} \mathrm{H} N M R,{ }^{13} \mathrm{C} N M R, H R-M S\right)$
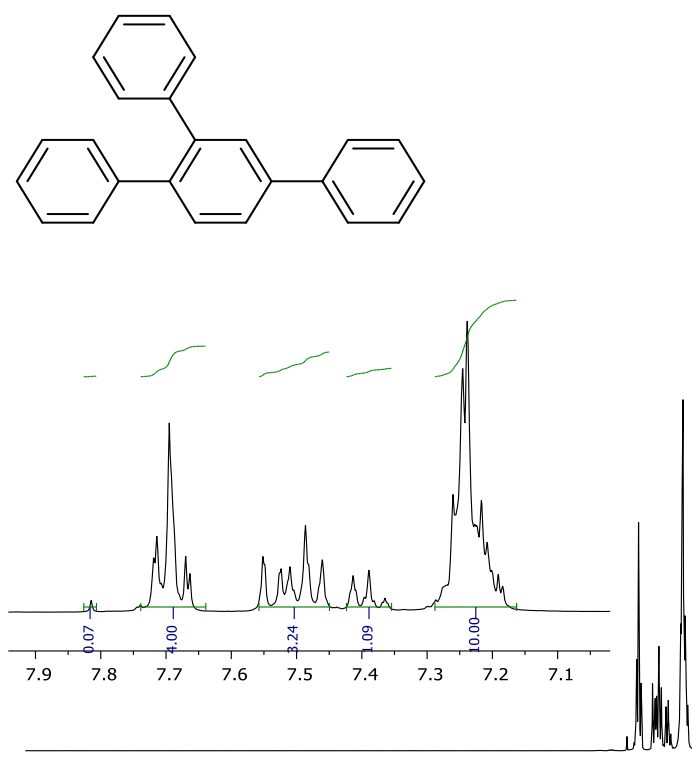

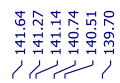

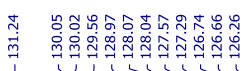

| Vरे परी

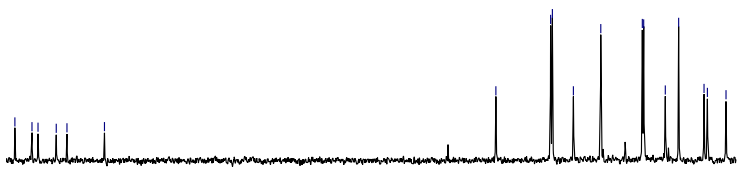

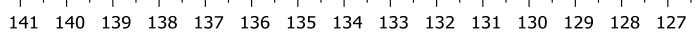

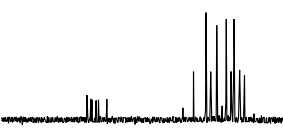

$\begin{array}{llllllllllllllllllllllllllllllllllllllllll}145 & 140 & 135 & 130 & 125 & 120 & 115 & 110 & 105 & 100 & 95 & 90 & 85 & 80 & 75 & 70 & 65 & 60 & 55 & 50 & 45 & 40 & 35 & 30 & 25 & 20 & 15 & 10 & 5 & 0\end{array}$ 


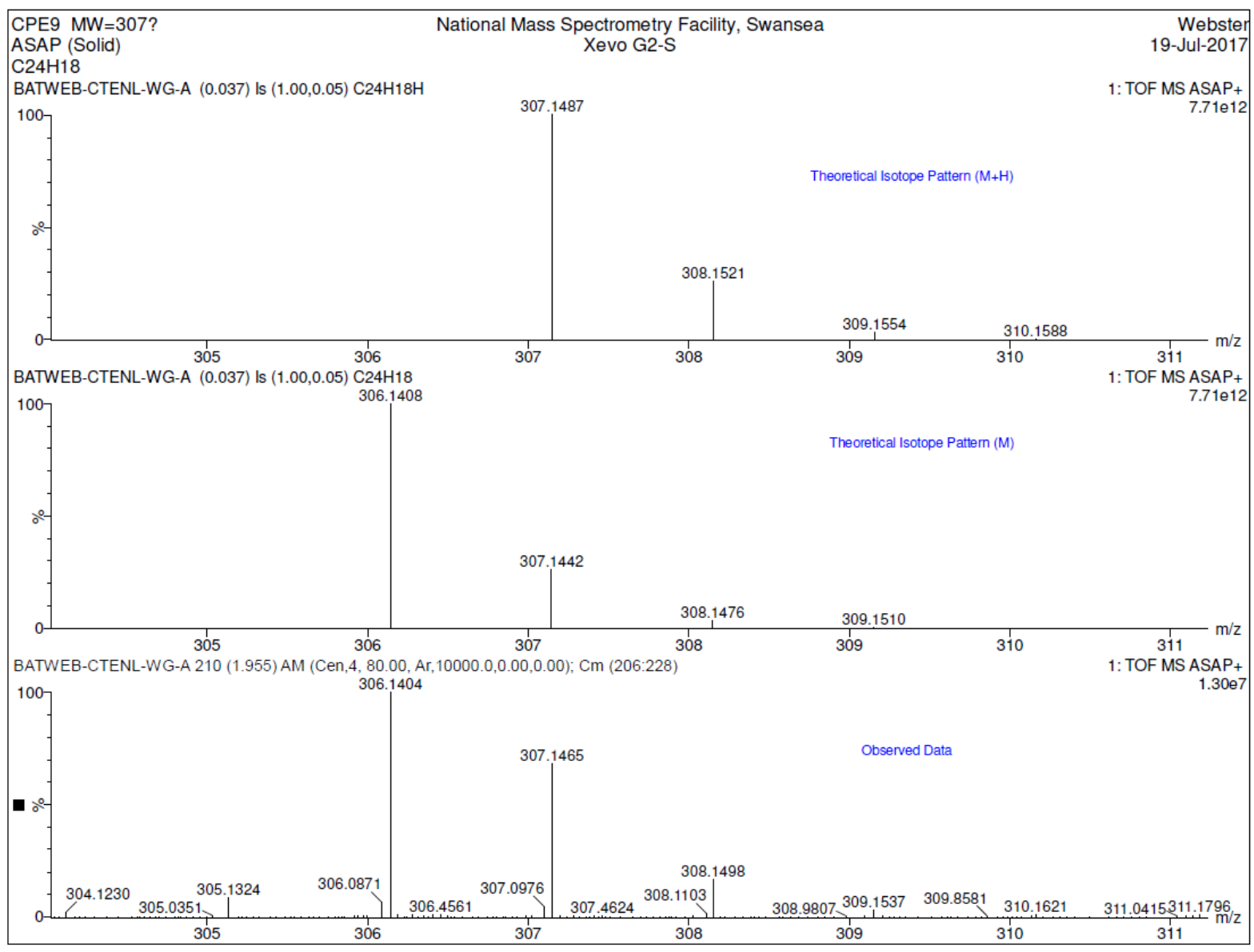

3b ( ${ }^{1} \mathrm{H}$ NMR, ${ }^{13} \mathrm{C}$ NMR, HR-MS)
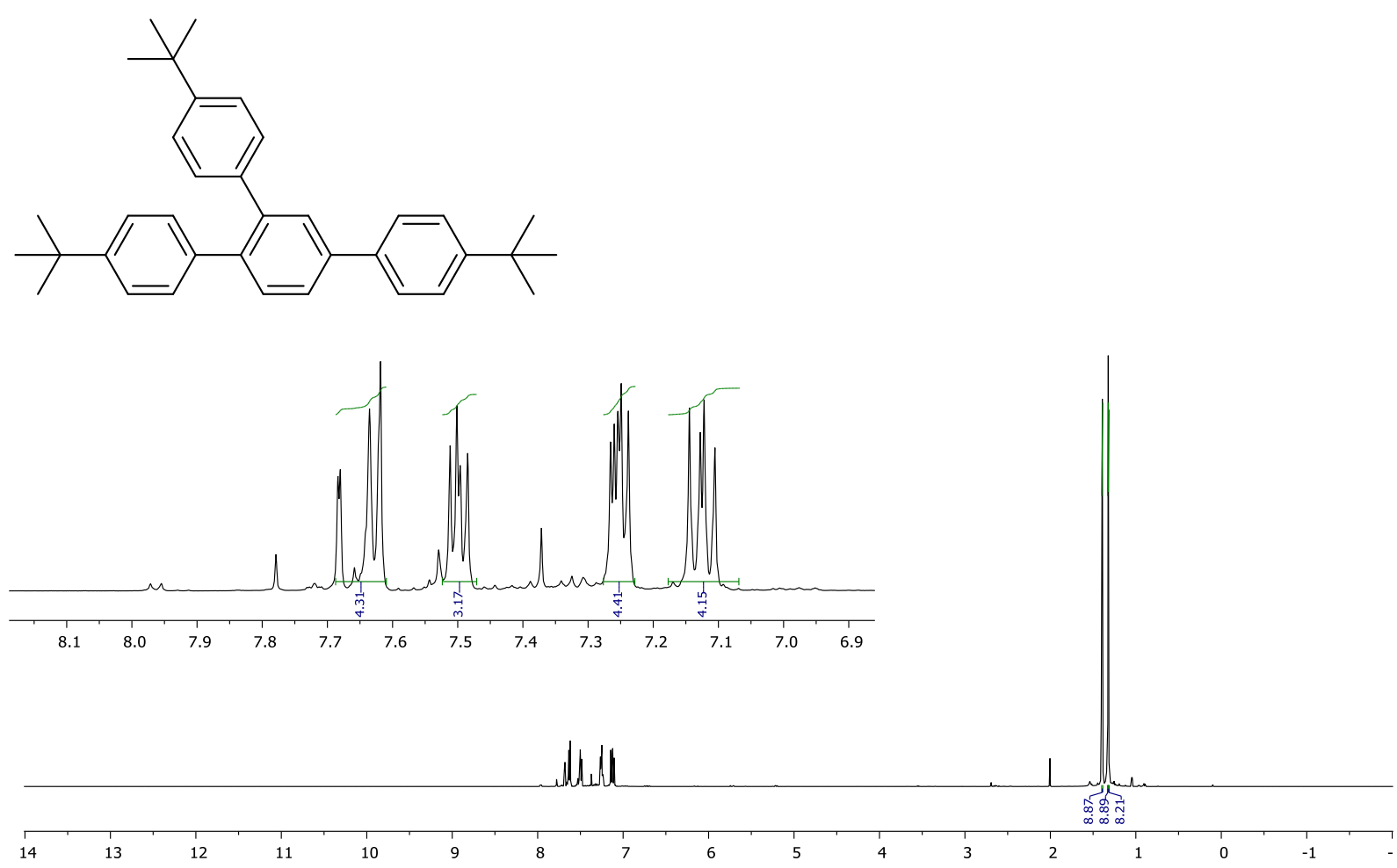

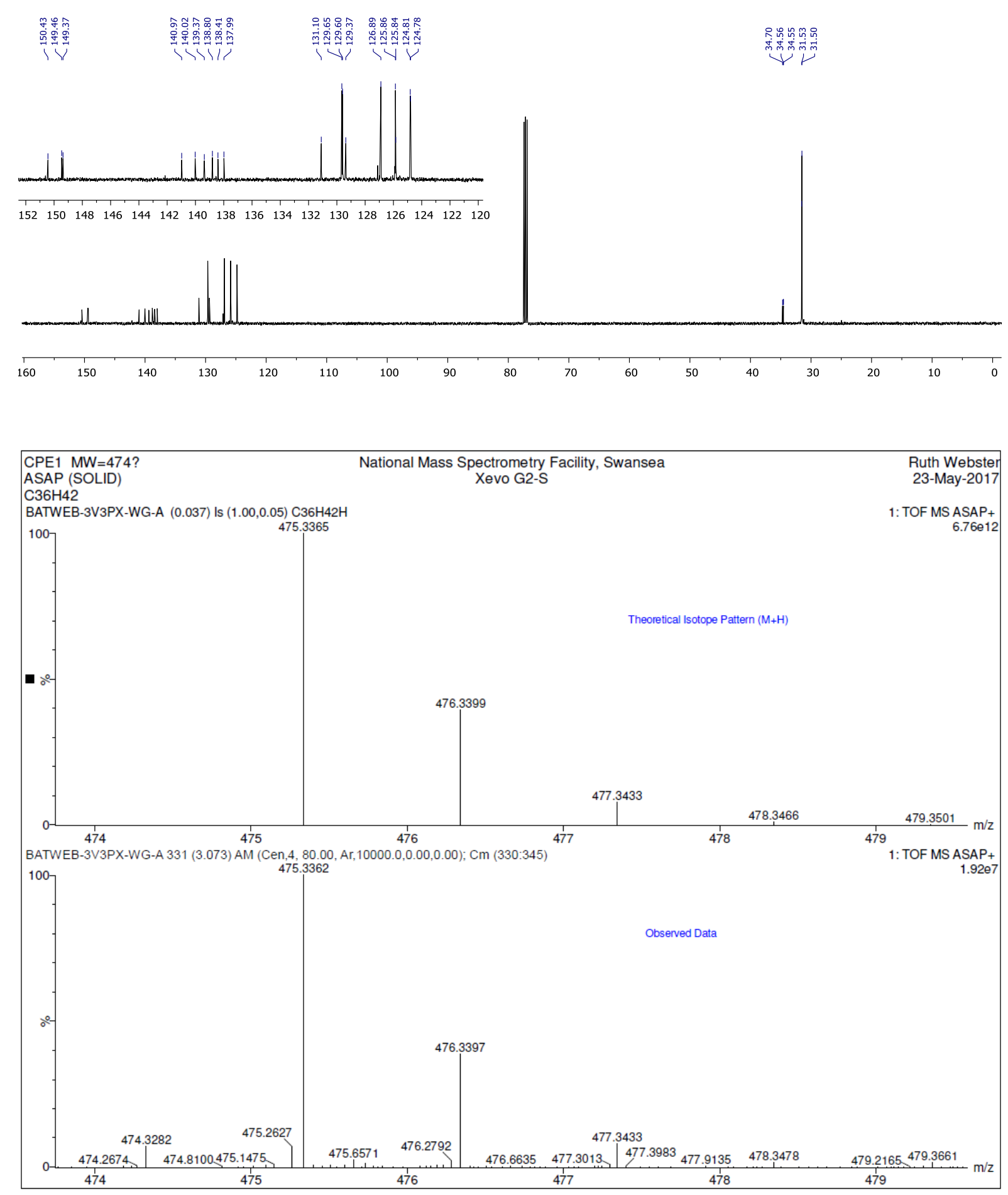
3c ( ${ }^{1} \mathrm{H} N M R,{ }^{13} \mathrm{C}$ NMR, HR-MS)
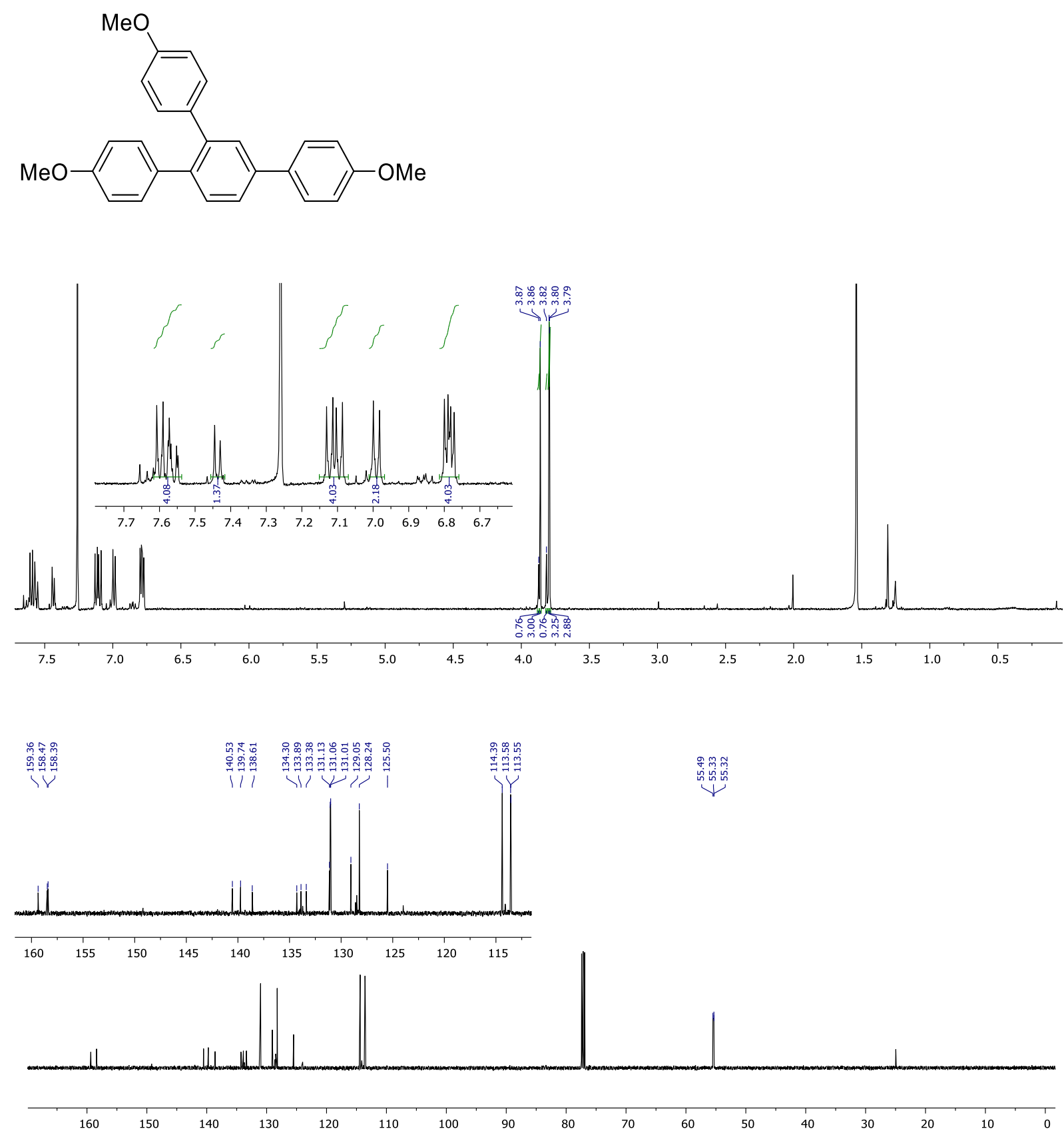


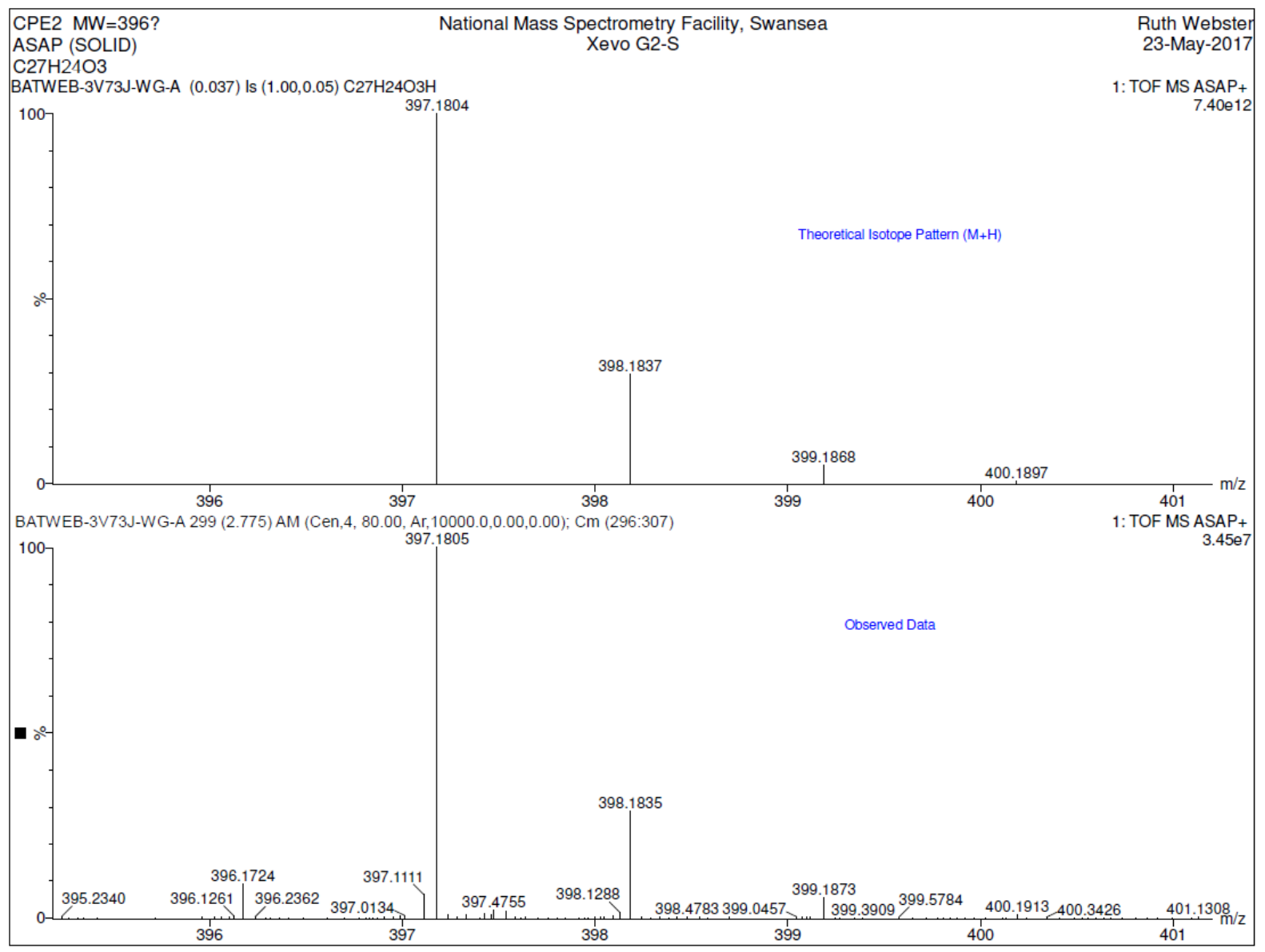

3d ( ${ }^{1} \mathrm{H}$ NMR, ${ }^{13} \mathrm{C}$ NMR, ${ }^{19} \mathrm{~F}$ NMR, HR-MS)
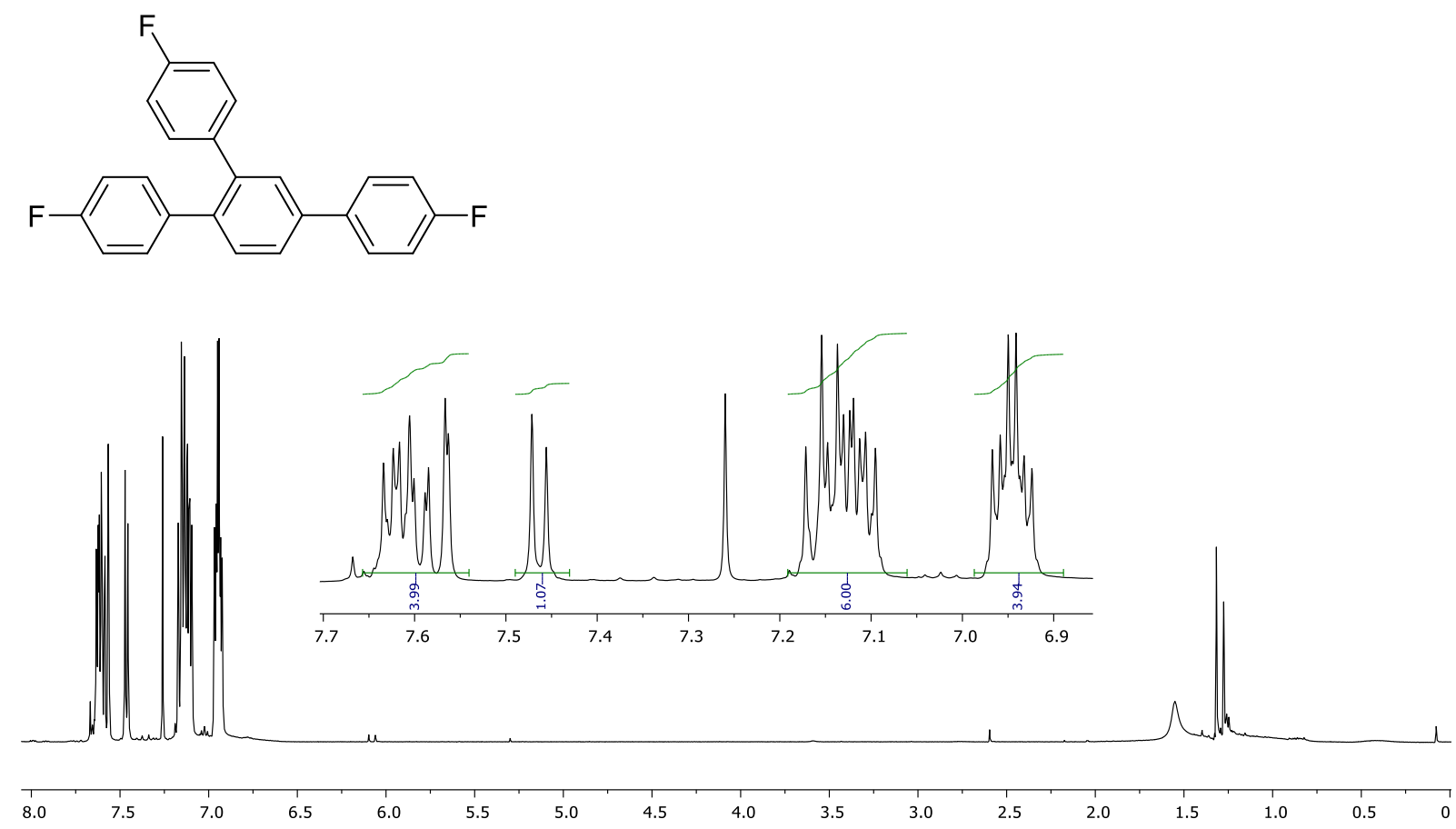

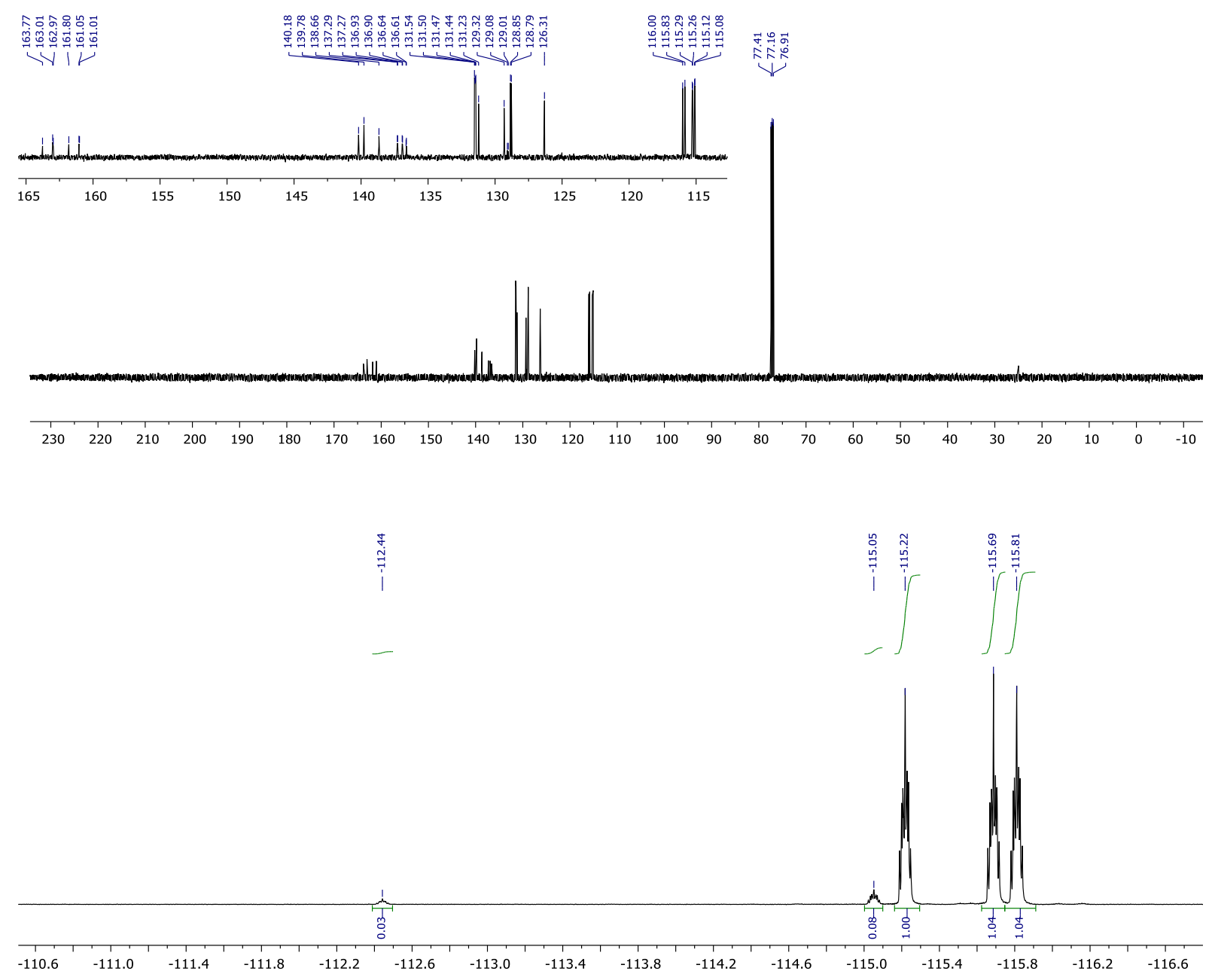
Supporting Information

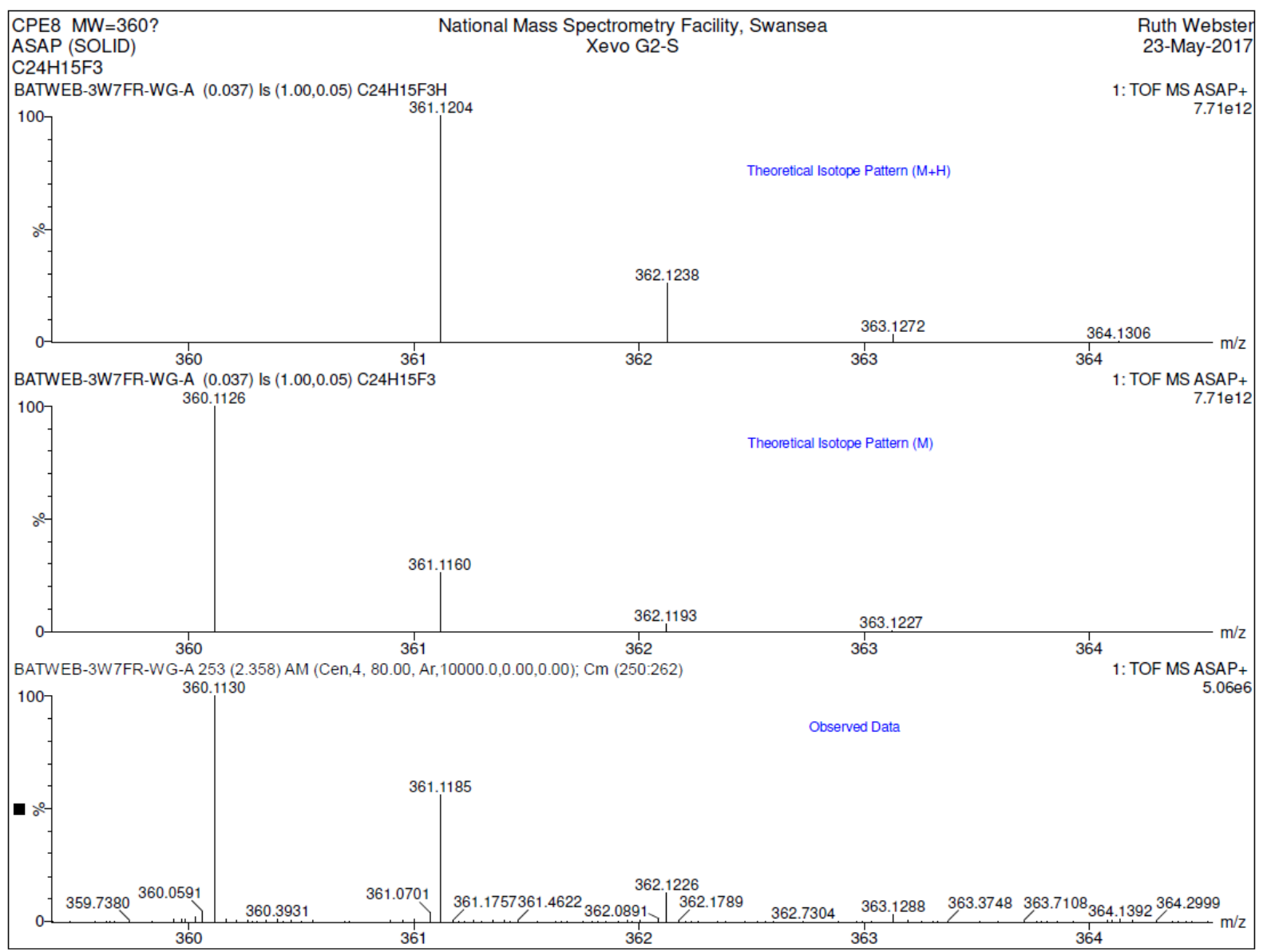

3e $\left({ }^{1} \mathrm{H} N M R,{ }^{13} \mathrm{C} N M R, F T I R, H R-M S\right)$<smiles>Cc1cccc(-c2ccc(-c3cccc(C)c3)c(-c3cccc(C)c3)c2)c1</smiles>
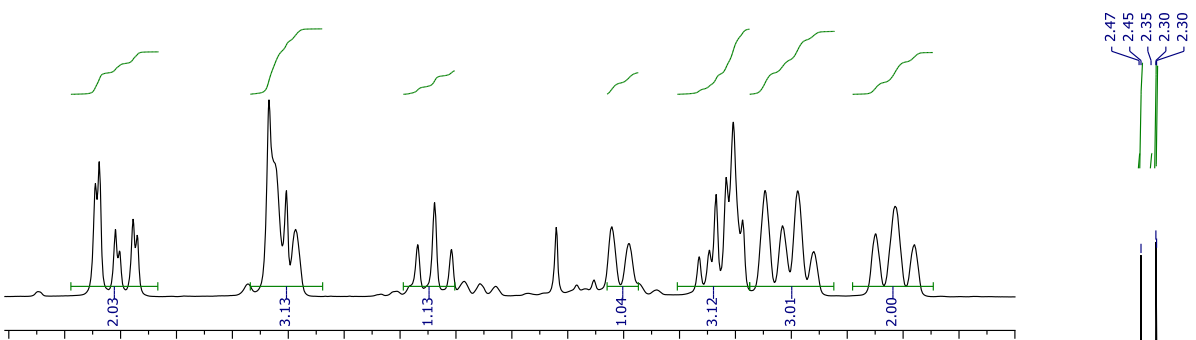

$\begin{array}{llllllllllllllllllll}.75 & 7.70 & 7.65 & 7.60 & 7.55 & 7.50 & 7.45 & 7.40 & 7.35 & 7.30 & 7.25 & 7.20 & 7.15 & 7.10 & 7.05 & 7.00 & 6.95 & 6.90 & 6.85\end{array}$
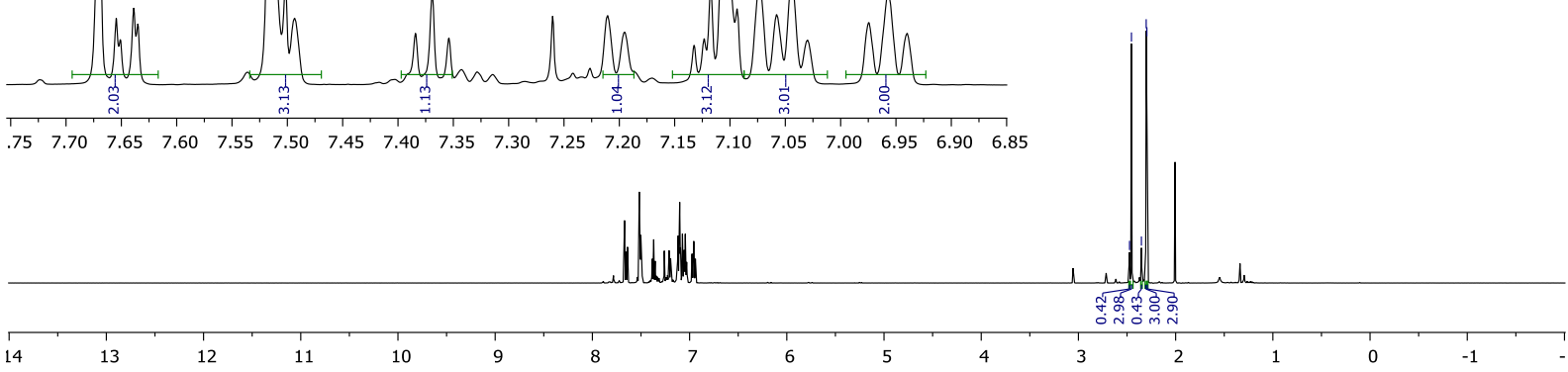


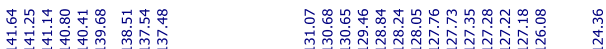

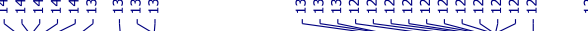

บูy
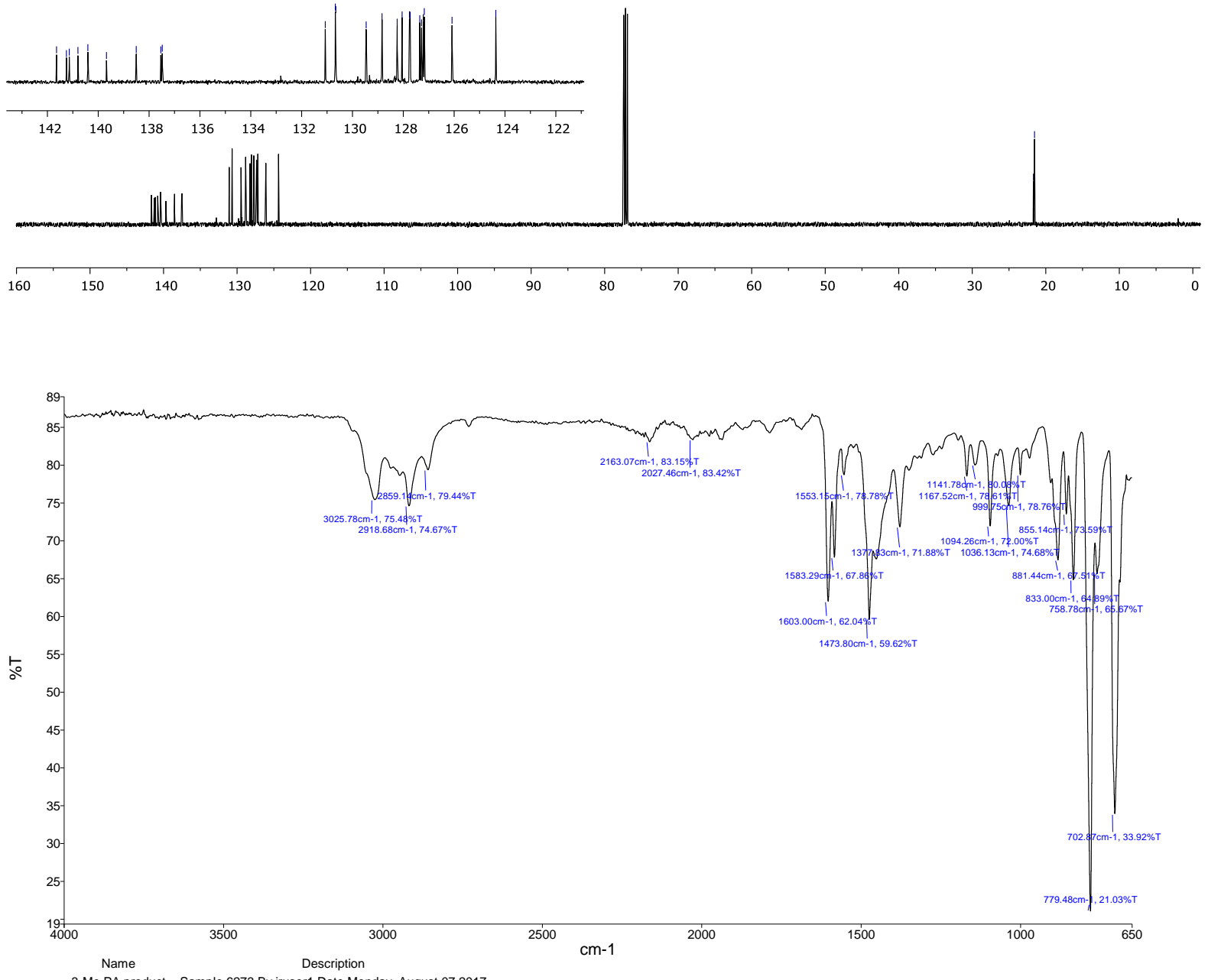

3-Me PA product Sample 6273 By iruser1 Date Monday, August 072017 


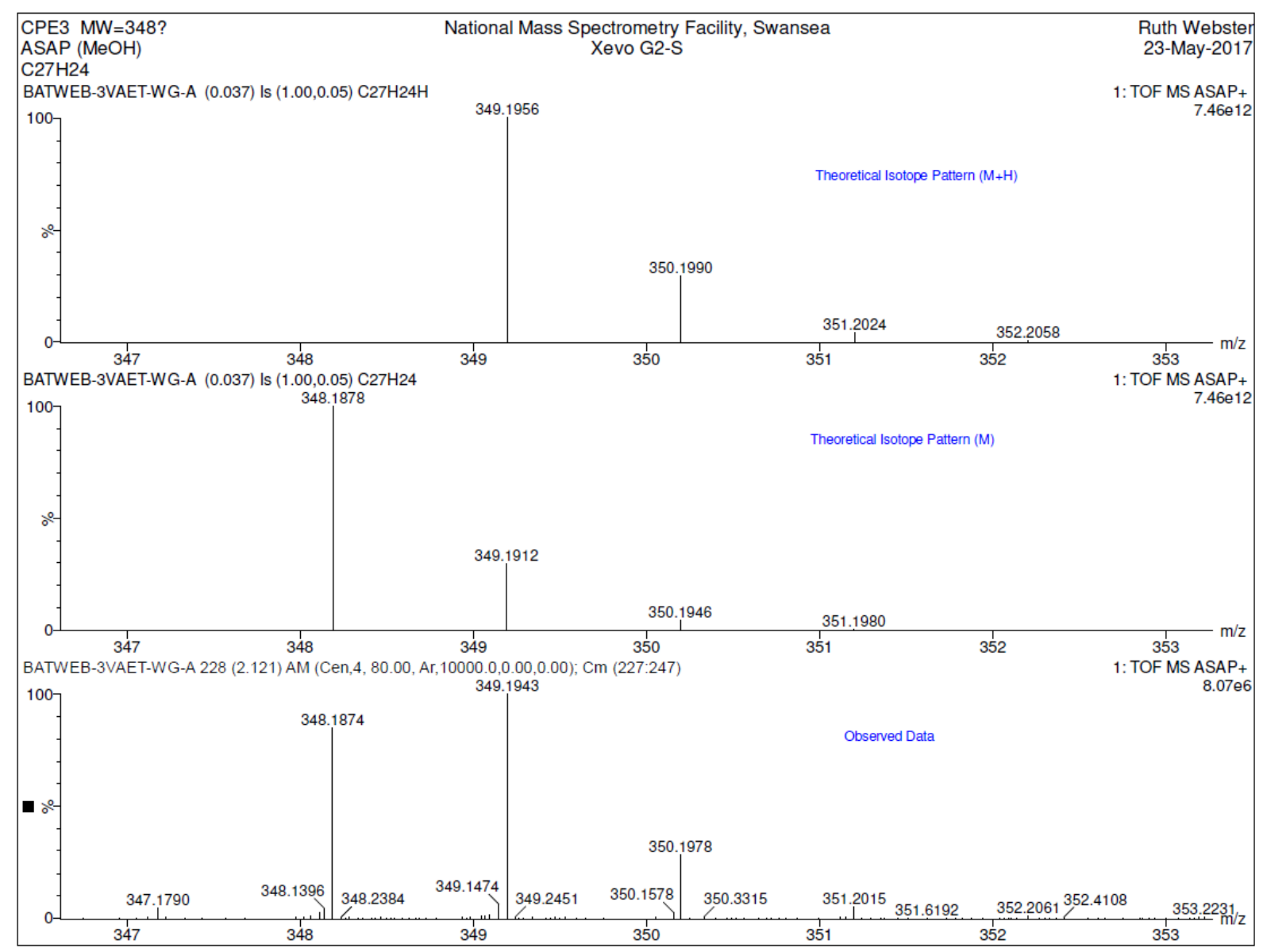

\section{$3 f\left({ }^{1} \mathrm{H}\right.$ NMR, ${ }^{13} \mathrm{C}$ NMR, FTIR HR-MS)}<smiles>Nc1ccccc1-c1ccc(-c2ccccc2N)c(-c2ccccc2N)c1</smiles>
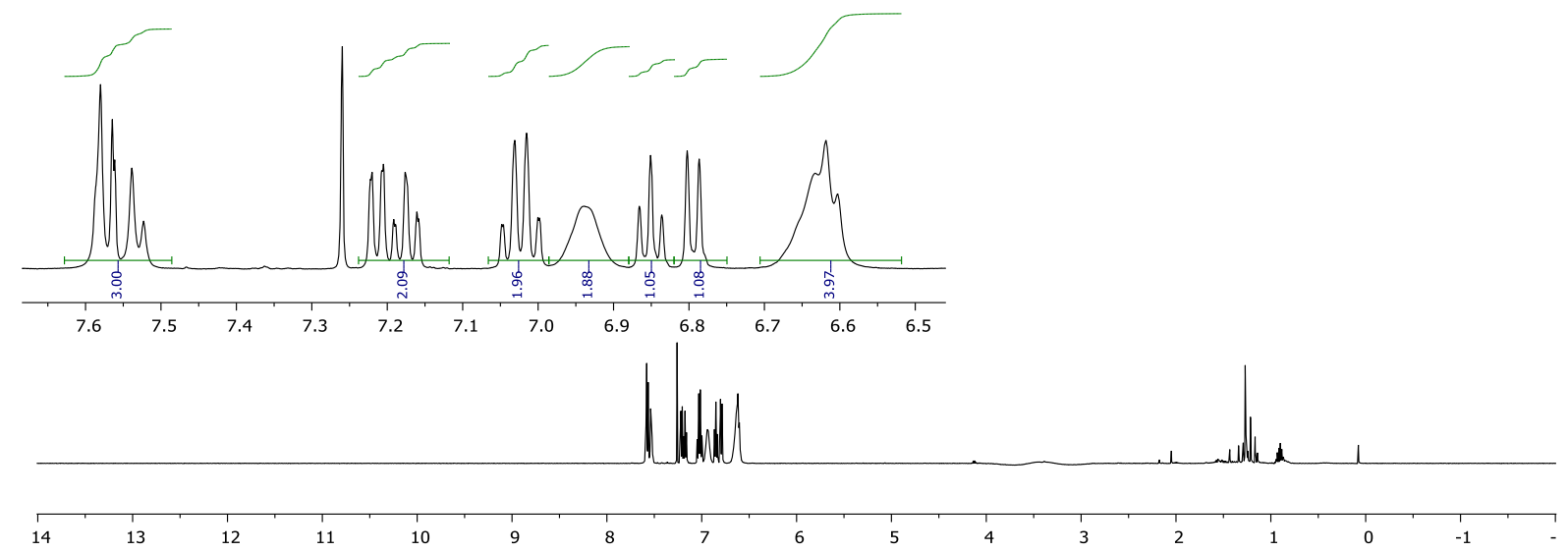


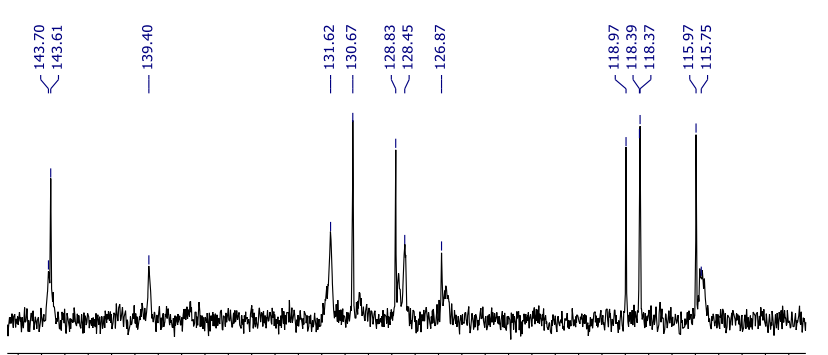

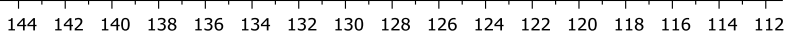
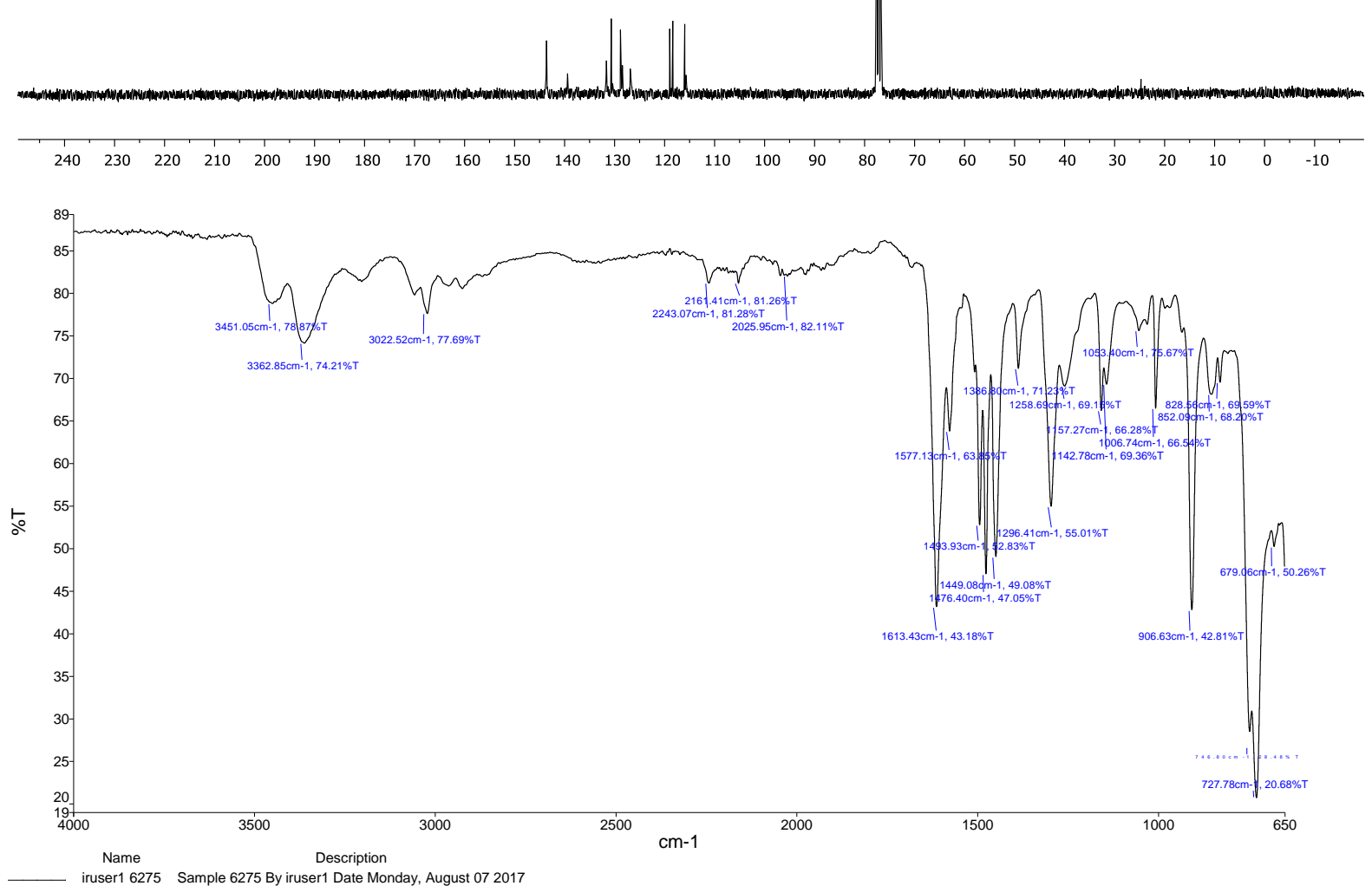

iruser1 6275 Sample 6275 By iruser1 Date Monday, August 072017 


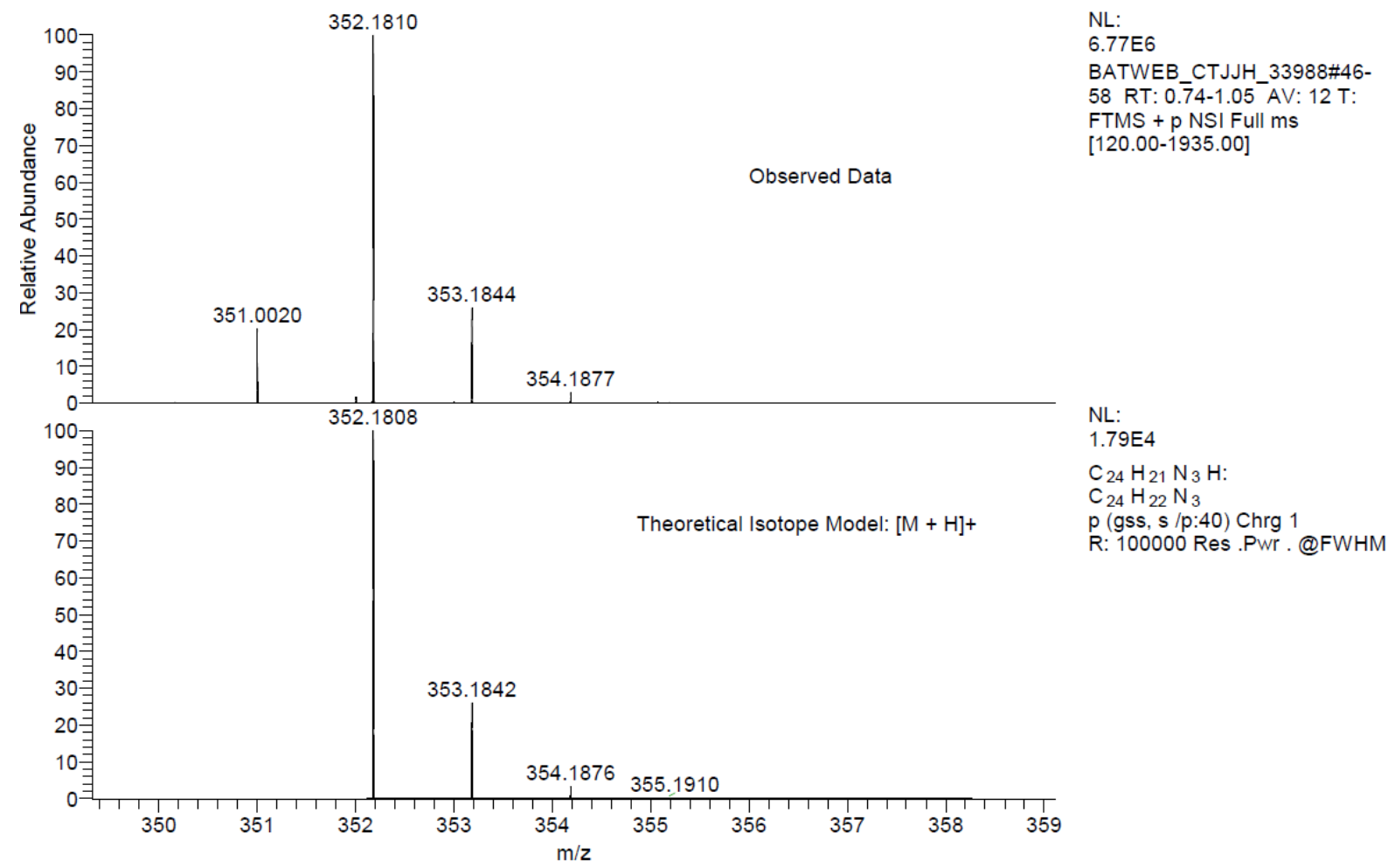

3g $\left({ }^{1} \mathrm{H}\right.$ NMR, ${ }^{13} \mathrm{C}$ NMR, FTIR, HR-MS)
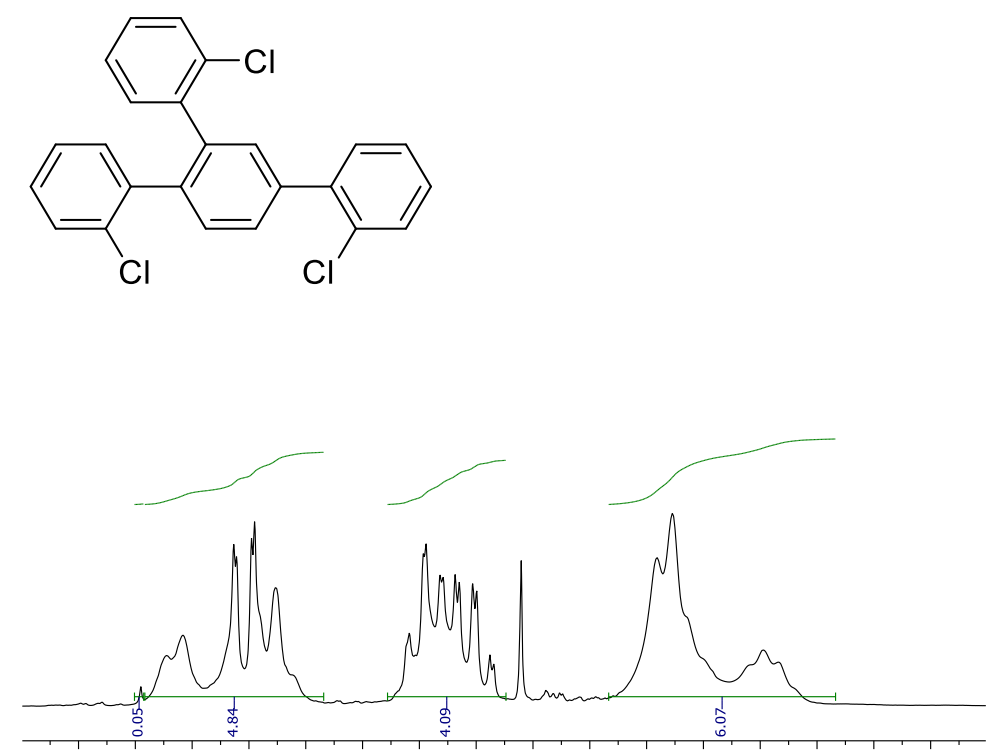

$\begin{array}{llllllllllllllll}7.65 & 7.60 & 7.55 & 7.50 & 7.45 & 7.40 & 7.35 & 7.30 & 7.25 & 7.20 & 7.15 & 7.10 & 7.05 & 7.00 & 6.95 & 6.90\end{array}$

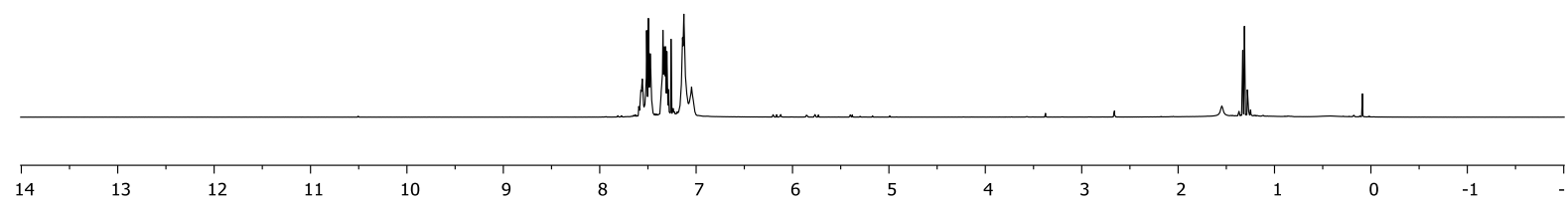



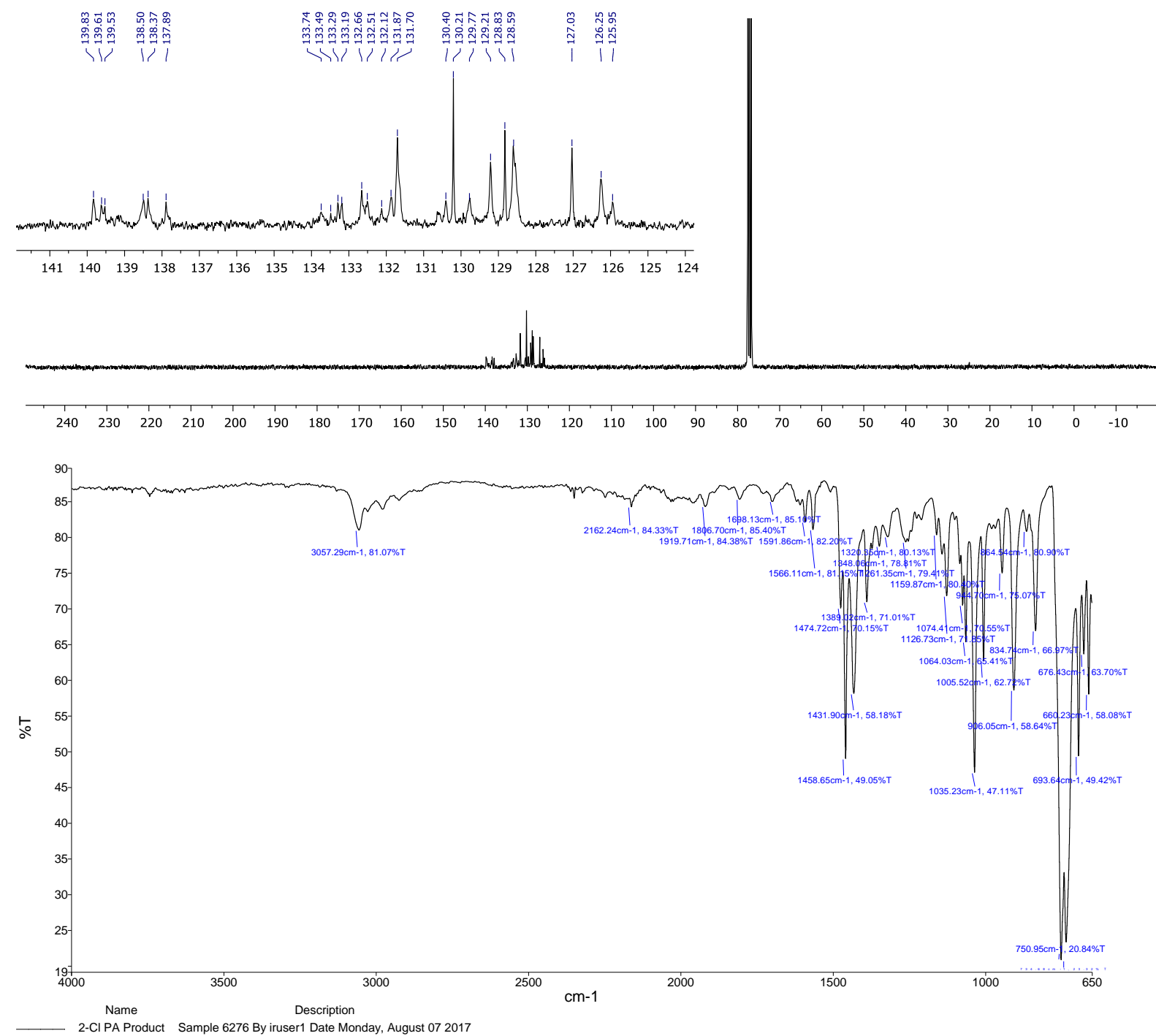
Supporting Information

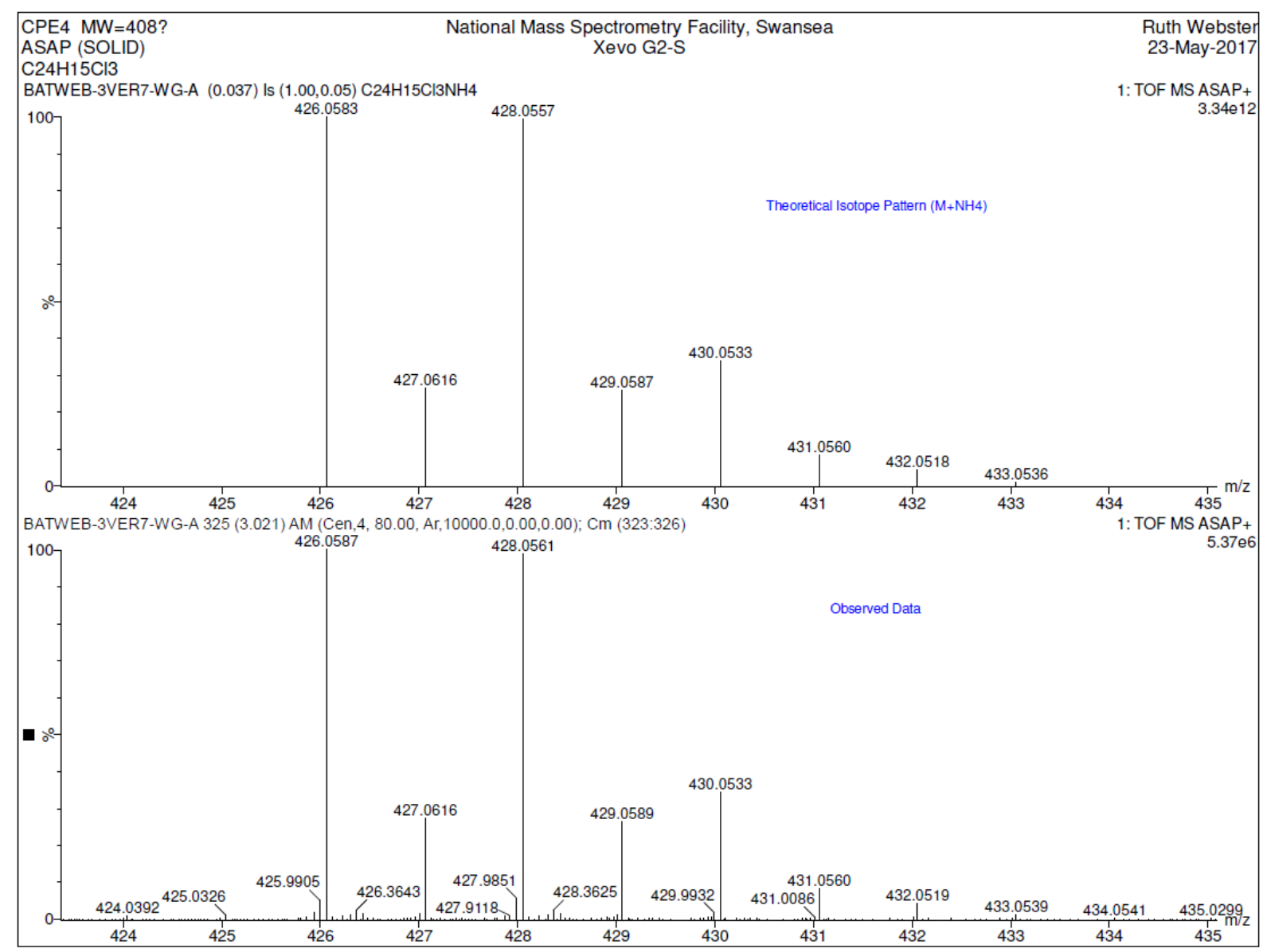

3h $\left({ }^{1} \mathrm{H}\right.$ NMR, ${ }^{13} \mathrm{C}$ NMR, FTIR HR-MS)

N=1>N>

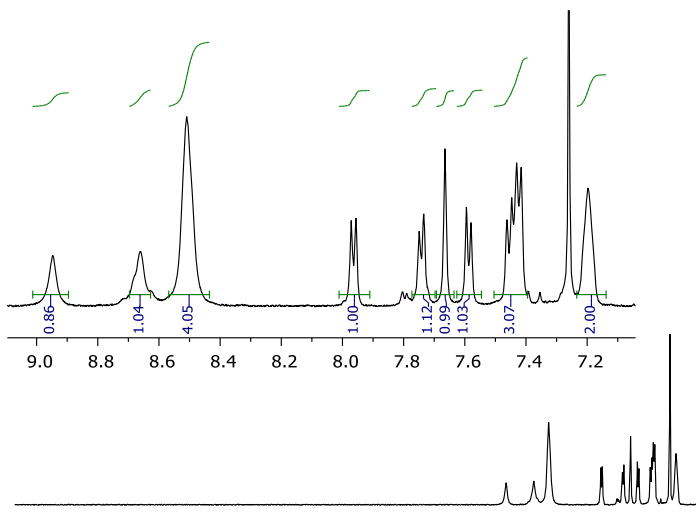

N

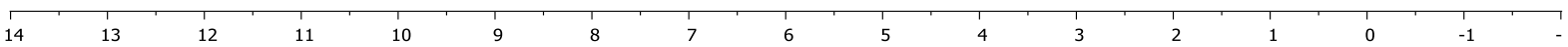

Page S52 

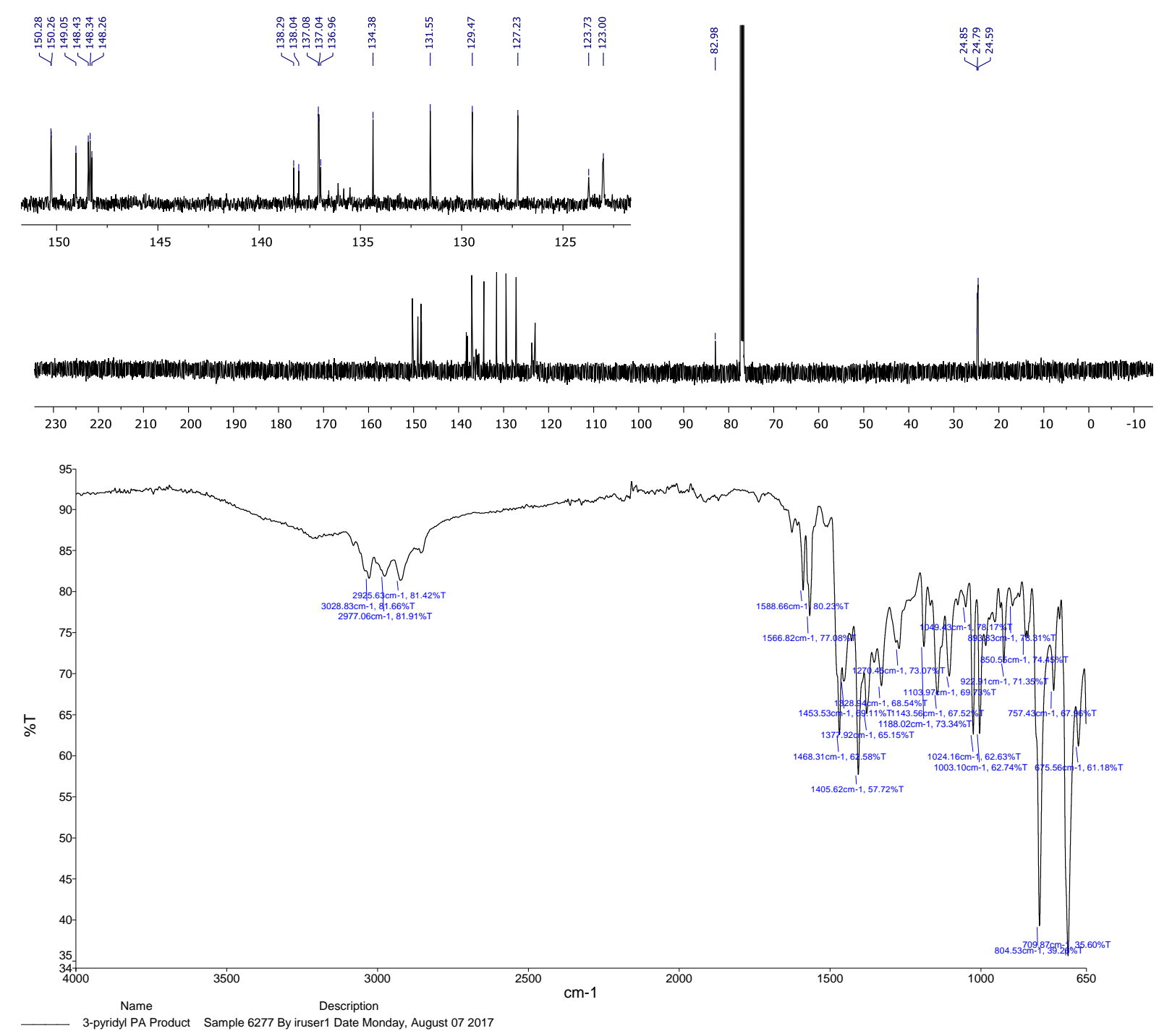


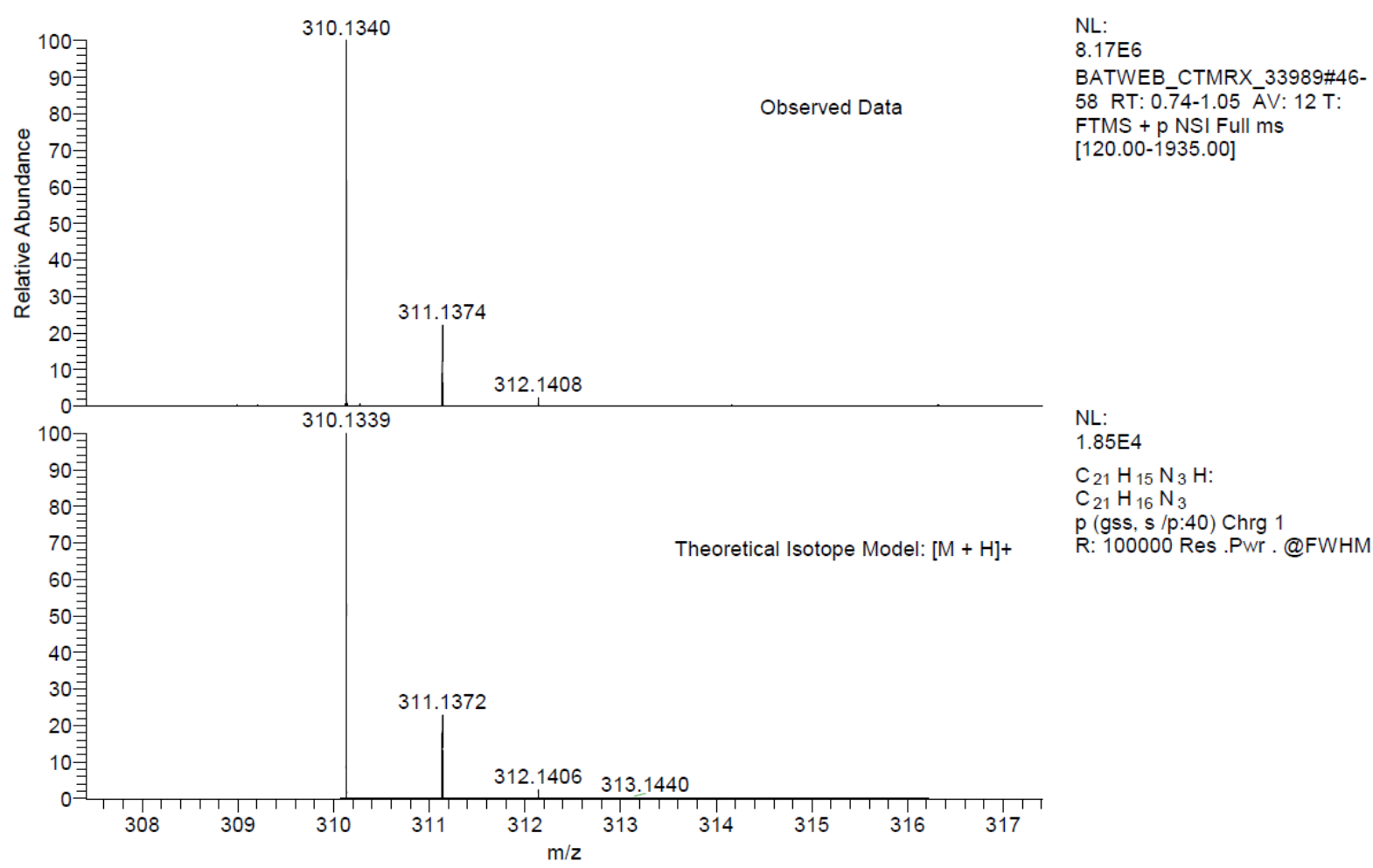

3i ( ${ }^{1} \mathrm{H}$ NMR, ${ }^{13} \mathrm{C}$ NMR, HR-MS)<smiles>CCOC(=O)c1ccc(C(=O)OCC)c(C(=O)OCC)c1</smiles>
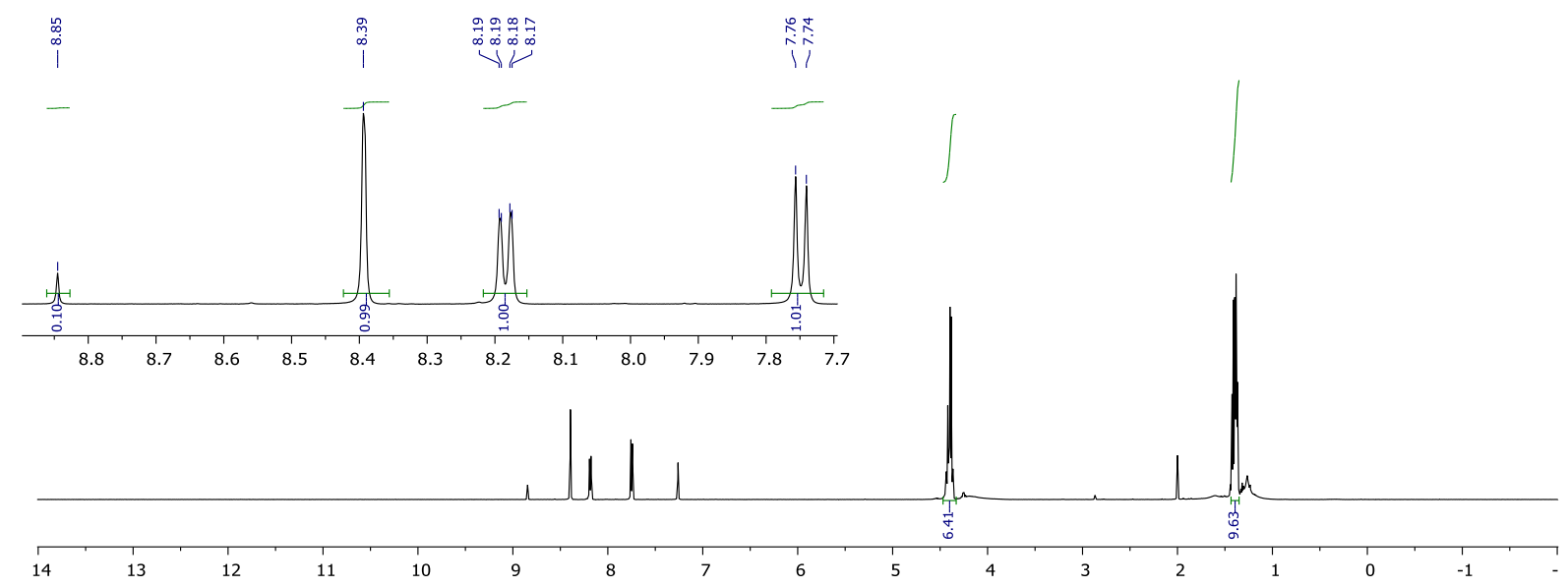


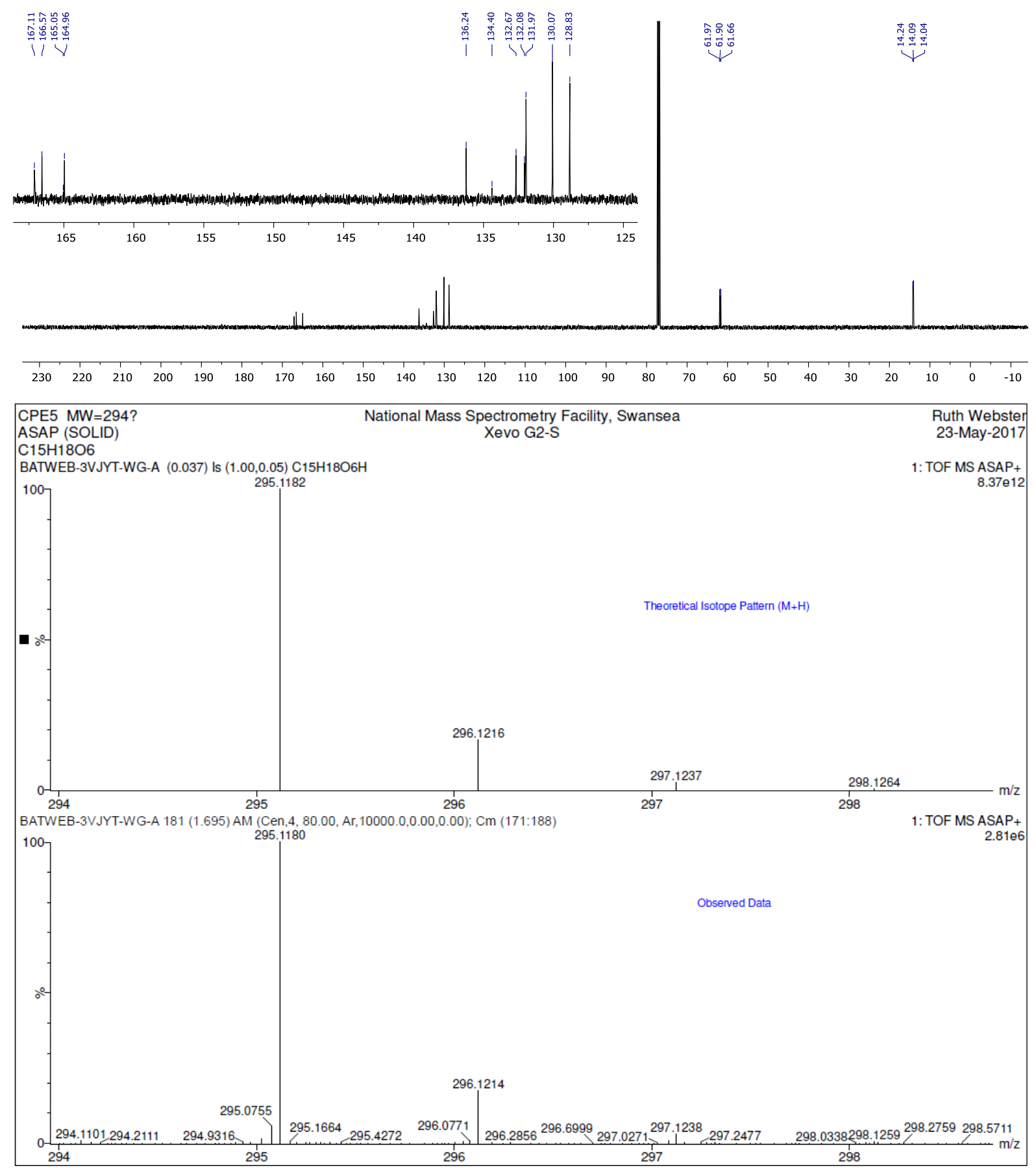

3j $\left({ }^{1} \mathrm{H}\right.$ NMR, ${ }^{13} \mathrm{C}$ NMR, HR-MS)<smiles>CCCCCc1ccc(CCCCC)c(CCCCC)c1</smiles><smiles>CCCCCc1cc(CCCCC)cc(CCCCC)c1</smiles> 

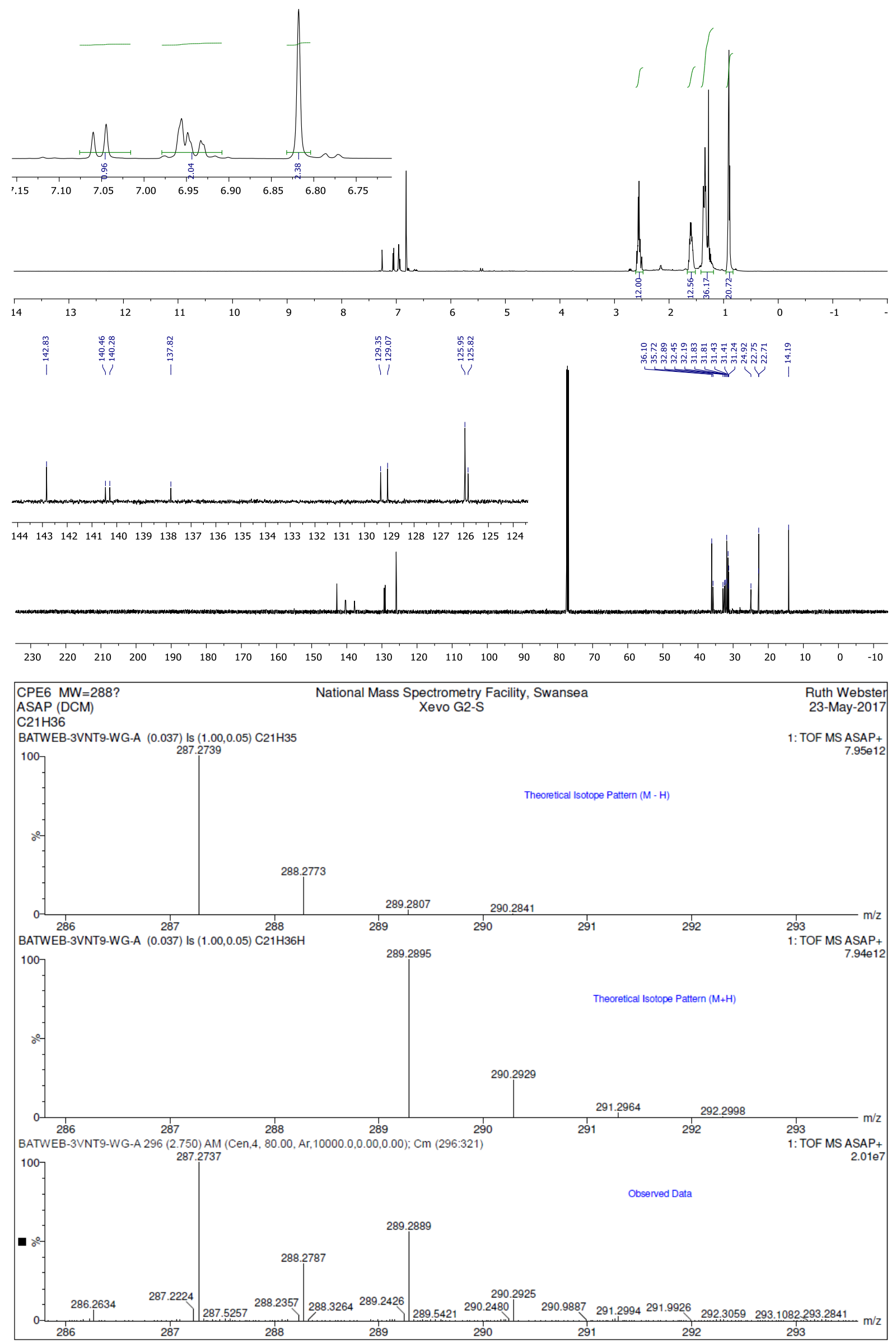
3k ( ${ }^{1} \mathrm{H}$ NMR, ${ }^{13} \mathrm{C}$ NMR, HR-MS)
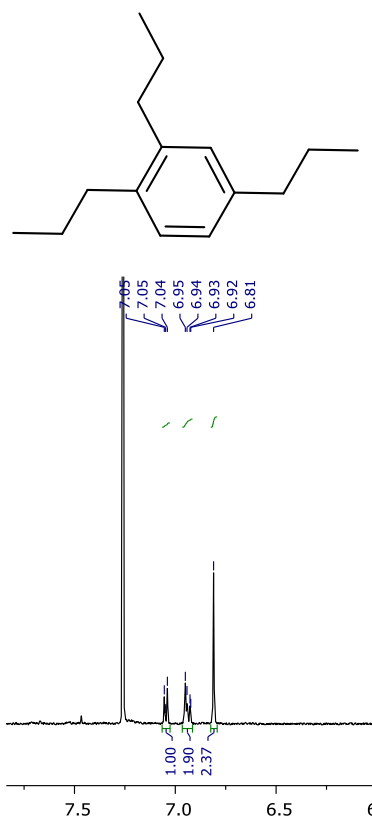<smiles>CCCc1cc(CCC)cc(CCC)c1</smiles>

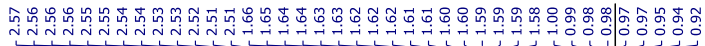
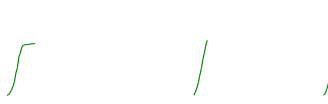

3I ( ${ }^{1} \mathrm{H} N M R,{ }^{13} \mathrm{C}$ NMR, HR-MS)

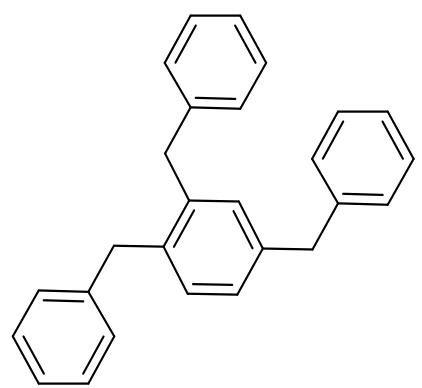

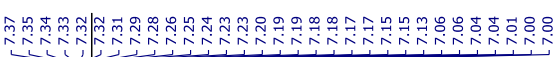

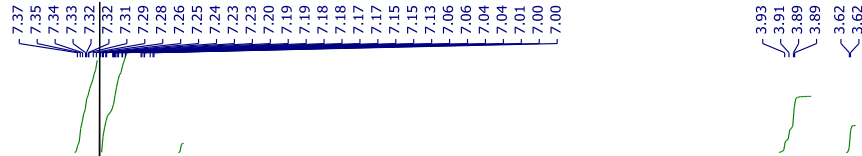

Page S57

$\sqrt{\min }$<smiles>c1ccc(Cc2cc(Cc3ccccc3)cc(Cc3ccccc3)c2)cc1</smiles>

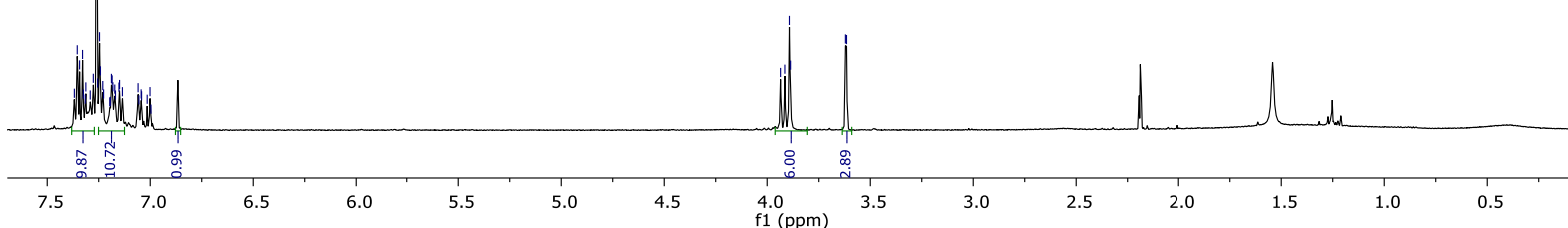




\section{Coordinates of Computed Species}

\section{FSPE: $-4654.43748640570 E_{\mathrm{h}}$}

\begin{tabular}{|c|c|c|c|}
\hline $\mathrm{Fe}$ & 5.195890000 & 4.706419000 & 2.622398000 \\
\hline 0 & 3.503127000 & 5.666649000 & 2.830444000 \\
\hline $\mathrm{N}$ & 4.887030000 & 7.357842000 & 5.164622000 \\
\hline$C$ & 7.931759000 & 4.213275000 & 1.906108000 \\
\hline-1 & 8.839573000 & 3.585393000 & 1.954148000 \\
\hline $\mathrm{Fe}$ & 6.223259000 & 6.066870000 & 5.594160000 \\
\hline $\mathrm{O}$ & 5.746086000 & 6.224839000 & 1.514097000 \\
\hline $\mathrm{N}$ & 7.476041000 & 7.304921000 & 4.829541000 \\
\hline C & 8.097115000 & 5.557122000 & 1.395588000 \\
\hline $\mathrm{O}$ & 7.594158000 & 4.724585000 & 6.009532000 \\
\hline $\mathrm{N}$ & 4.628276000 & 3.234619000 & 3.684551000 \\
\hline C & 9.409501000 & 5.961717000 & 1.025271000 \\
\hline $\mathrm{H}$ & 10.216199000 & 5.221000000 & 1.103051000 \\
\hline $\mathrm{O}$ & 5.013058000 & 4.810736000 & 6.433017000 \\
\hline$N$ & 6.818862000 & 3.717174000 & 2.352233000 \\
\hline C & 9.699865000 & 7.263358000 & 0.614397000 \\
\hline $\mathrm{H}$ & 10.725456000 & 7.550226000 & 0.352998000 \\
\hline C & 8.646025000 & 8.208915000 & 0.555640000 \\
\hline $\mathrm{H}$ & 8.854915000 & 9.244534000 & 0.254557000 \\
\hline C & 7.339026000 & 7.837792000 & 0.874140000 \\
\hline $\mathrm{H}$ & 6.517793000 & 8.564304000 & 0.821433000 \\
\hline C & 7.007387000 & 6.504971000 & 1.282755000 \\
\hline $\mathrm{O}$ & 6.018376000 & 5.409563000 & 3.994839000 \\
\hline C & 6.745658000 & 2.372200000 & 2.933507000 \\
\hline $\mathrm{H}$ & 7.743725000 & 2.010575000 & 3.242759000 \\
\hline $\mathrm{H}$ & 6.333477000 & 1.683412000 & 2.170411000 \\
\hline C & 5.783532000 & 2.454032000 & 4.127071000 \\
\hline $\mathrm{H}$ & 5.493128000 & 1.450203000 & 4.488685000 \\
\hline $\mathrm{H}$ & 6.259104000 & 3.030089000 & 4.945152000 \\
\hline C & 3.408763000 & 2.949161000 & 4.024129000 \\
\hline $\mathrm{H}$ & 3.235556000 & 2.036154000 & 4.620150000 \\
\hline C & 2.251609000 & 3.747618000 & 3.685927000 \\
\hline C & 0.964445000 & 3.227335000 & 3.988529000 \\
\hline $\mathrm{H}$ & 0.905368000 & 2.233924000 & 4.454900000 \\
\hline C & -0.205658000 & 3.939208000 & 3.719301000 \\
\hline $\mathrm{H}$ & -1.186848000 & 3.512320000 & 3.959471000 \\
\hline C & -0.098436000 & 5.233739000 & 3.156383000 \\
\hline $\mathrm{H}$ & -1.005933000 & 5.817334000 & 2.948685000 \\
\hline C & 1.150325000 & 5.787169000 & 2.871871000 \\
\hline $\mathrm{H}$ & 1.238412000 & 6.798073000 & 2.453015000 \\
\hline C & 2.367089000 & 5.073422000 & 3.115309000 \\
\hline C & 8.661577000 & 7.001673000 & 4.397642000 \\
\hline $\mathrm{H}$ & 9.217992000 & 7.763456000 & 3.822629000 \\
\hline C & 9.303870000 & 5.716913000 & 4.570937000 \\
\hline C & 10.565884000 & 5.519863000 & 3.945978000 \\
\hline $\mathrm{H}$ & 11.003242000 & 6.361770000 & 3.393454000 \\
\hline C & 11.229331000 & 4.292489000 & 3.980896000 \\
\hline $\mathrm{H}$ & 12.192154000 & 4.163900000 & 3.472140000 \\
\hline C & 10.628264000 & 3.213686000 & 4.676053000 \\
\hline $\mathrm{H}$ & 11.123843000 & 2.233625000 & 4.700455000 \\
\hline C & 9.410031000 & 3.382261000 & 5.335797000 \\
\hline $\mathrm{H}$ & 8.944198000 & 2.551951000 & 5.882143000 \\
\hline C & 8.711021000 & 4.632947000 & 5.326845000 \\
\hline C & 3.719798000 & 4.980539000 & 6.555509000 \\
\hline C & 2.965516000 & 3.932686000 & 7.176090000 \\
\hline $\mathrm{H}$ & 3.521710000 & 3.051377000 & 7.520401000 \\
\hline C & 1.579967000 & 4.004651000 & 7.308012000 \\
\hline $\mathrm{H}$ & 1.037947000 & 3.167541000 & 7.768626000 \\
\hline C & 0.864893000 & 5.131819000 & 6.833937000 \\
\hline $\mathrm{H}$ & -0.227856000 & 5.171379000 & 6.906603000 \\
\hline
\end{tabular}




$\begin{array}{llll}\text { C } & 1.573459000 & 6.173783000 & 6.238810000 \\ \text { H } & 1.034181000 & 7.043004000 & 5.839229000 \\ \text { C } & 2.989235000 & 6.141279000 & 6.093275000 \\ \text { C } & 3.614868000 & 7.233193000 & 5.387683000 \\ \text { H } & 2.940719000 & 8.002960000 & 4.971632000 \\ \text { C } & 5.400520000 & 8.349436000 & 4.212614000 \\ \text { H } & 4.801902000 & 9.279138000 & 4.221776000 \\ \text { H } & 5.344973000 & 7.878065000 & 3.210661000 \\ \text { C } & 6.865903000 & 8.612238000 & 4.568347000 \\ \text { H } & 7.389390000 & 9.150592000 & 3.756718000 \\ \text { H } & 6.926020000 & 9.214483000 & 5.495747000 \\ \text { C } & 6.558959000 & 7.386154000 & 8.403949000 \\ \text { N } & 6.469382000 & 6.879205000 & 7.361990000 \\ \text { C } & 6.664830000 & 8.014372000 & 9.708847000 \\ \text { H } & 6.790883000 & 9.105271000 & 9.593201000 \\ \text { H } & 7.535202000 & 7.608516000 & 10.253900000 \\ \text { H } & 5.750312000 & 7.818333000 & 10.295839000 \\ \text { C } & 3.895779000 & 3.338335000 & 0.148948000 \\ \text { N } & 4.400561000 & 3.864635000 & 1.053419000 \\ \text { C } & 3.263210000 & 2.680168000 & -0.980210000 \\ \text { H } & 3.648685000 & 1.650275000 & -1.079767000 \\ \text { H } & 3.478059000 & 3.235991000 & -1.909808000 \\ \text { H } & 2.169860000 & 2.641673000 & -0.830660000\end{array}$

\section{I FSPE: -2157.25477458926 $E_{\mathrm{h}}$}

\begin{tabular}{|c|c|c|c|}
\hline & 5.028991000 & 4.100893000 & 1.939981000 \\
\hline o & 3.258740000 & 4.287134000 & 1.364427000 \\
\hline & 7.818173000 & 4.720995000 & 2.134293000 \\
\hline & 8.845976000 & 4.397383000 & 2.368131000 \\
\hline & 5.274887000 & 5.777185000 & 1.145464000 \\
\hline & 7.634553000 & 6.071652000 & 1.685153000 \\
\hline & 4.800600000 & 2.361825000 & 2.567175000 \\
\hline & 8.751333000 & 6.958692000 & 1.670077000 \\
\hline & 9.715573000 & 6.591393000 & 2.046613000 \\
\hline & 6.852027000 & 3.836768000 & 2.250891000 \\
\hline & 8.637047000 & 8.262006000 & 1.191440000 \\
\hline & 9.505497000 & 8.931410000 & 1.193988000 \\
\hline & 7.386182000 & 8.713371000 & 0.694343000 \\
\hline & 7.287927000 & 9.738001000 & 0.312690000 \\
\hline & 6.276781000 & 7.868042000 & 0.684405000 \\
\hline & 5.306743000 & 8.208154000 & 0.300649000 \\
\hline & 6.361730000 & 6.535279000 & 1.186015000 \\
\hline & 7.203911000 & 2.441731000 & 2.600478000 \\
\hline & 8.100990000 & 2.399910000 & 3.242994000 \\
\hline & 7.418836000 & 1.902189000 & 1.658922000 \\
\hline & 5.984488000 & 1.837280000 & 3.280926000 \\
\hline & 6.010975000 & 0.733500000 & 3.266375000 \\
\hline & 5.923470000 & 2.176804000 & 4.331625000 \\
\hline & 3.706012000 & 1.632138000 & 2.529315000 \\
\hline & 2.459927000 & 2.059708000 & 1.963111000 \\
\hline & 1.354176000 & 1.157606000 & 1.956875000 \\
\hline & 1.492793000 & 0.159101000 & 2.393051000 \\
\hline & 0.124313000 & 1.519815000 & 1.413400000 \\
\hline & -0.713800000 & 0.812810000 & 1.417280000 \\
\hline & -0.034195000 & 2.815079000 & 0.852963000 \\
\hline & -1.000424000 & 3.108421000 & 0.422257000 \\
\hline & 1.024382000 & 3.722610000 & 0.845753000 \\
\hline & 0.909684000 & 4.726368000 & 0.417616000 \\
\hline & 2.294325000 & 3.378865000 & 1.398977000 \\
\hline & 3.746325000 & 0.619748000 & 2.964067000 \\
\hline & 4.897251000 & 4.619981000 & 3.317417000 \\
\hline
\end{tabular}


1II FSPE: $-4314.53442020515 E_{\mathrm{h}}$

$\begin{array}{llll}\mathrm{Fe} & 5.100006000 & 4.102033000 & 1.512390000\end{array}$

$\begin{array}{lllll}\text { O } & 3.471360000 & 4.391499000 & 0.573672000\end{array}$

$\begin{array}{llll}\text { C } & 7.857648000 & 4.454368000 & 2.230651000\end{array}$

$\begin{array}{llll}\text { H } & 8.786693000 & 4.060372000 & 2.674581000\end{array}$

$\begin{array}{llll}\text { O } & 5.682309000 & 5.698293000 & 0.690295000\end{array}$

$\begin{array}{llll}\text { C } \quad 7.857349000 & 5.821574000 & 1.792935000\end{array}$

$\begin{array}{llll}\mathrm{N} & 4.589011000 & 2.399283000 & 2.150376000\end{array}$

C $\quad 8.995865000 \quad 6.632599000 \quad 2.069686000$

$\begin{array}{llll}\mathrm{H} & 9.816606000 & 6.194656000 & 2.653288000\end{array}$

$\begin{array}{llll}\mathrm{N} & 6.835265000 & 3.642353000 & 2.118550000\end{array}$

$\begin{array}{llll}\text { C } & 9.079122000 & 7.949393000 & 1.622061000\end{array}$

$\begin{array}{llll}\text { H } & 9.958518000 & 8.561449000 & 1.855614000\end{array}$

$\begin{array}{llll}\text { C } & 8.015504000 & 8.485879000 & 0.849402000\end{array}$

$\begin{array}{llll}\text { H } & 8.071993000 & 9.521230000 & 0.488054000\end{array}$

$\begin{array}{llll}\text { C } & 6.897065000 & 7.712113000 & 0.542434000\end{array}$

$\begin{array}{llll}\text { H } & 6.067175000 & 8.116994000 & -0.048419000\end{array}$

$\begin{array}{llll}\text { C } & 6.770545000 & 6.372071000 & 1.016510000\end{array}$

$\begin{array}{llll}\text { C } & 6.990390000 & 2.215136000 & 2.464248000\end{array}$

$\begin{array}{llll}\text { H } & 7.767651000 & 2.070542000 & 3.236294000\end{array}$

$\begin{array}{llll}\text { H } & 7.301677000 & 1.680555000 & 1.547102000\end{array}$

$\begin{array}{llll}\text { C } & 5.633964000 & 1.703732000 & 2.935185000\end{array}$

$\begin{array}{llll}\text { H } & 5.553018000 & 0.607227000 & 2.834239000\end{array}$

$\begin{array}{llll}\text { H } & 5.481649000 & 1.968565000 & 3.997592000\end{array}$

$\begin{array}{llll}\text { C } & 3.381453000 & 1.885210000 & 2.112746000\end{array}$

C $\quad 2.230945000 \quad 2.526029000 \quad 1.536407000$

$\begin{array}{llll}\text { C } & 0.948902000 & 1.929663000 & 1.723581000\end{array}$

$\begin{array}{llll}\mathrm{H} & 0.886126000 & 0.998836000 & 2.303521000\end{array}$

$\begin{array}{llll}\text { C } & -0.206559000 & 2.510978000 & 1.205340000\end{array}$

$\begin{array}{llll}\text { H } & -1.185558000 & 2.047617000 & 1.375693000\end{array}$

$\begin{array}{llll}\text { C } & -0.100918000 & 3.708635000 & 0.450873000\end{array}$

$\begin{array}{llll}\text { H } & -1.005184000 & 4.175890000 & 0.039406000\end{array}$

$\begin{array}{llll}\text { C } & 1.139768000 & 4.306385000 & 0.231553000\end{array}$

$\begin{array}{llll}\text { H } & 1.230946000 & 5.241064000 & -0.334196000\end{array}$

$\begin{array}{llll}\text { C } & 2.333957000 & 3.753644000 & 0.782179000\end{array}$

$\begin{array}{llll}\text { H } & 3.225134000 & 0.900508000 & 2.585281000\end{array}$

$\begin{array}{llll}\text { H } & 5.057463000 & 4.473392000 & 3.342982000\end{array}$

Fe $\quad 4.307781000 \quad 5.599441000 \quad 3.987492000$

$\begin{array}{llll}\text { O } & 6.038738000 & 6.078737000 & 4.603333000\end{array}$

$\begin{array}{llll}\text { C } & 1.673843000 & 6.159546000 & 3.0006661000\end{array}$

$\begin{array}{llll}\text { H } & 0.593987000 & 5.938855000 & 2.972493000\end{array}$

$\begin{array}{llll}\text { O } & 4.353161000 & 7.350197000 & 3.234354000\end{array}$

$\begin{array}{llll}\text { C } & 2.159865000 & 7.227534000 & 2.166672000\end{array}$

$\begin{array}{llll}\text { N } & 4.137099000 & 4.136243000 & 5.166551000\end{array}$

$\begin{array}{llll}\text { C } & 1.284333000 & 7.796333000 & 1.198407000\end{array}$

$\begin{array}{llll}\text { H } & 0.292406000 & 7.343440000 & 1.064537000\end{array}$

$\begin{array}{llll}\mathrm{N} & 2.426072000 & 5.458212000 & 3.809986000\end{array}$

$\begin{array}{llll}\text { C } & 1.660116000 & 8.898750000 & 0.430722000\end{array}$

$\begin{array}{llll}\text { H } & 0.977205000 & 9.312198000 & -0.321192000\end{array}$

$\begin{array}{llll}\text { C } & 2.932491000 & 9.487771000 & 0.647470000\end{array}$

$\begin{array}{llll}\text { H } & 3.237022000 & 10.362438000 & 0.057415000\end{array}$

$\begin{array}{llll}\text { C } \quad 3.807014000 & 8.965831000 & 1.601860000\end{array}$

$\begin{array}{llll}\text { H } & 4.795665000 & 9.409169000 & 1.770295000\end{array}$

$\begin{array}{llll}\text { C } & 3.469725000 & 7.807562000 & 2.364533000\end{array}$

$\begin{array}{llll}\text { C } & 1.783044000 & 4.509455000 & 4.743029000\end{array}$

$\begin{array}{llll}H & 0.889379000 & 4.045484000 & 4.286909000\end{array}$

H $\quad \begin{array}{llll}\text { H } & 1.466410000 & 5.076697000 & 5.638931000\end{array}$

$\begin{array}{llll}\text { C } & 2.822221000 & 3.466687000 & 5.121913000\end{array}$

$\begin{array}{llll}\text { H } & 2.583623000 & 2.975841000 & 6.081974000\end{array}$

H $\quad 2.877593000 \quad 2.688575000 \quad 4.339644000$

C $\quad 5.094717000 \quad 3.598973000 \quad 5.884995000$

C $\quad 6.465825000 \quad 4.039261000 \quad 5.873571000$

$\begin{array}{llll}\text { C } & 7.446938000 & 3.241585000 & 6.531524000\end{array}$

$\begin{array}{llll}\text { H } & 7.124947000 & 2.302401000 & 7.002084000\end{array}$

C $8.788218000 \quad 3.621335000 \quad 6.575985000$

Page $\mathbf{S 6 0}$ 


$\begin{array}{cccc}\text { H } & 9.530163000 & 2.982844000 & 7.070238000 \\ \mathrm{C} & 9.178295000 & 4.848561000 & 5.981283000 \\ \mathrm{H} & 10.230149000 & 5.161939000 & 6.013391000 \\ \mathrm{C} & 8.239200000 & 5.662780000 & 5.347021000 \\ \mathrm{H} & 8.532479000 & 6.606858000 & 4.873146000 \\ \mathrm{C} & 6.869382000 & 5.276744000 & 5.244305000 \\ \mathrm{H} & 4.842417000 & 2.733770000 & 6.520577000 \\ \mathrm{H} & 4.100662000 & 5.263305000 & 2.551476000\end{array}$

\section{${ }^{34}$ FSPE: -2156.669238386694 $E_{\mathrm{h}}$}

\begin{tabular}{|c|c|c|c|}
\hline & & & \\
\hline & & & \\
\hline & 0 & 00 & 00 \\
\hline & 00 & 00 & 000 \\
\hline & 000 & 00 & 000 \\
\hline & 56000 & 6.07 & 3000 \\
\hline & 4.795190000 & 2.364778000 & .560853000 \\
\hline & 8.785171000 & 6.94 & 4000 \\
\hline & 9.750941000 & 6.5561 & 2.000528000 \\
\hline & 6.866192000 & 000 & 4000 \\
\hline & 8.680747000 & 8.26 & 2000 \\
\hline & 9.5 & & 000 \\
\hline & 7.423144000 & 8.74897 & 000 \\
\hline & 7.329 & 9.78 & 1000 \\
\hline & 6.300940000 & 7.9207 & 000 \\
\hline & 5.326731000 & 8.28760 & 778000 \\
\hline & 6.377939000 & 6.5666 & 000 \\
\hline & 7.203743000 & 2.44 & 2.5 \\
\hline & 8.10 & 2.4 & 000 \\
\hline & 7.40 & 1.8 & 1.0 \\
\hline & & & \\
\hline & 6.005867000 & 0.7638 & 358000 \\
\hline & 5.920986000 & 2.2496 & 47000 \\
\hline & 96000 & 1.6 & 2.5 \\
\hline & 2.450367000 & 2.04 & 2000 \\
\hline & 1.347444000 & 1.1440 & 8000 \\
\hline & 1.493502000 & 0.1577 & 2.453883000 \\
\hline & 0.111441000 & 1.48697 & 68000 \\
\hline & -0.723739000 & 0.77700 & 540000 \\
\hline & -0.055393000 & 2.768892000 & 0.863488000 \\
\hline & -1.025999000 & 3.049751000 & 0.433979000 \\
\hline & 1.000047000 & 3.67995 & 0.825928000 \\
\hline & 0.876468000 & 4.672893000 & 0.375402000 \\
\hline & 2.278562000 & 3.354311000 & 1.370935000 \\
\hline & 3.725678000 & 0.657068000 & 3.04492400 \\
\hline
\end{tabular}

\section{4-MeCN \\ Fe $\quad 4.959878000$ \\ O 3.178832000 \\ C $\quad 7.684989000$ \\ H 8.720151000 \\ O 5.153030000 \\ C 7.388273000 \\ N 4.832541000 \\ C 8.372029000 \\ H 9.318179000 \\ N 6.805963000 \\ C 8.150195000 \\ H 8.912380000 \\ C $\quad 6.926460000$ \\ H 6.739722000 \\ C $\quad 5.952847000$ \\ $4.347172000 \quad 2.041931000$ \\ $4.481920000 \quad 1.401295000$ \\ $5.099473000 \quad 2.529356000$ \\ $4.827695000 \quad 2.797505000$ \\ $6.144804000 \quad 1.436410000$ \\ $6.498273000 \quad 2.361215000$ \\ $2.515703000 \quad 2.452536000$ \\ $7.458755000 \quad 2.738066000$ \\ $7.095429000 \quad 3.162176000$ \\ $4.137756000 \quad 2.388384000$ \\ $8.827351000 \quad 2.597958000$ \\ $9.549450000 \quad 2.914016000$ \\ $9.274468000 \quad 2.035573000$ \\ $10.350016000 \quad 1.918395000$ \\ $8.360318000 \quad 1.629723000$}

FSPE: -2289.521067389265 $E_{\mathrm{h}}$

Page S61 


$\begin{array}{llll}\text { H } & 5.003047000 & 8.700194000 & 1.197614000 \\ \text { C } & 6.135439000 & 6.953503000 & 1.795407000 \\ \text { C } & 7.228537000 & 2.729815000 & 2.516346000 \\ \text { H } & 8.135869000 & 2.629536000 & 3.139054000 \\ \text { H } & 7.451142000 & 2.342683000 & 1.503724000 \\ \text { C } & 6.041448000 & 1.979911000 & 3.109907000 \\ \text { H } & 6.133401000 & 0.887048000 & 2.974686000 \\ \text { H } & 5.964304000 & 2.197779000 & 4.192298000 \\ \text { C } & 3.792135000 & 1.731092000 & 2.282730000 \\ \text { H } & 3.884899000 & 0.675232000 & 2.589177000 \\ \text { C } & 2.535263000 & 2.144298000 & 1.721499000 \\ \text { C } & 1.498863000 & 1.174205000 & 1.573033000 \\ \text { H } & 1.698076000 & 0.145891000 & 1.905319000 \\ \text { C } & 0.261116000 & 1.502265000 & 1.024060000 \\ \text { H } & -0.521258000 & 0.740723000 & 0.921313000 \\ \text { C } & 0.026218000 & 2.835841000 & 0.597970000 \\ \text { H } & -0.944680000 & 3.107338000 & 0.162757000 \\ \text { C } & 1.016807000 & 3.809342000 & 0.727519000 \\ \text { H } & 0.840621000 & 4.842533000 & 0.401464000 \\ \text { C } & 2.292481000 & 3.505633000 & 1.294961000 \\ \text { C } & 5.161588000 & 6.082441000 & 4.672249000 \\ \text { N } & 4.675060000 & 5.181392000 & 4.109559000 \\ \text { C } & 5.798735000 & 7.221252000 & 5.314087000 \\ \text { H } & 5.848338000 & 8.072884000 & 4.567907000 \\ \text { H } & 6.856130000 & 6.941135000 & 5.624871000 \\ \text { H } & 5.201384000 & 7.533469000 & 6.225029000\end{array}$

\section{${ }^{15}$ FSPE: $-2568.834448357149 E_{\mathrm{h}}$}

$\begin{array}{llll}\mathrm{Fe} & 4.962164000 & 4.582716000 & 3.374454000 \\ \mathrm{O} & 2.805988000 & 5.568708000 & 6.266527000 \\ \mathrm{C} & 7.459700000 & 3.440193000 & 2.779535000 \\ \mathrm{H} & 8.232737000 & 2.686087000 & 2.991910000 \\ \mathrm{O} & 5.667327000 & 5.666119000 & 2.081776000 \\ \mathrm{C} & 7.709659000 & 4.380797000 & 1.713391000 \\ \mathrm{~N} & 4.439877000 & 3.950302000 & 5.259587000 \\ \mathrm{C} & 8.914289000 & 4.265276000 & 0.961778000 \\ \mathrm{H} & 9.605796000 & 3.447901000 & 1.206377000 \\ \mathrm{~N} & 6.378817000 & 3.432081000 & 3.529881000 \\ \mathrm{C} & 9.224428000 & 5.159136000 & -0.064350000 \\ \mathrm{H} & 10.155704000 & 5.046864000 & -0.632059000 \\ \mathrm{C} & 8.327000000 & 6.212768000 & -0.363158000 \\ \mathrm{H} & 8.561261000 & 6.920603000 & -1.168635000 \\ \mathrm{C} & 7.138395000 & 6.361211000 & 0.357995000 \\ \mathrm{H} & 6.437255000 & 7.175411000 & 0.137256000 \\ \mathrm{C} & 6.800078000 & 5.456424000 & 1.398425000 \\ \mathrm{C} & 6.194974000 & 2.360920000 & 4.548435000 \\ \mathrm{H} & 7.166609000 & 1.940233000 & 4.860084000 \\ \mathrm{H} & 5.606452000 & 1.554812000 & 4.073011000 \\ \mathrm{C} & 5.455689000 & 2.972093000 & 5.739184000 \\ \mathrm{H} & 4.966013000 & 2.195808000 & 6.353390000 \\ \mathrm{H} & 6.146557000 & 3.541010000 & 6.379494000 \\ \mathrm{C} & 3.459056000 & 3.526187000 & 4.375171000 \\ \mathrm{C} & 2.200979000 & 4.276125000 & 4.305187000 \\ \mathrm{C} & 1.229845000 & 3.982148000 & 3.315877000 \\ \mathrm{H} & 1.456926000 & 3.207030000 & 2.572110000 \\ \mathrm{C} & 0.005652000 & 4.660100000 & 3.286364000 \\ \mathrm{H} & -0.736334000 & 4.425384000 & 2.513822000 \\ \mathrm{C} & -0.268293000 & 5.643313000 & 4.263790000 \\ \mathrm{H} & -1.226715000 & 6.177733000 & 4.250454000 \\ \mathrm{C} & 0.676373000 & 5.947758000 & 5.254747000 \\ \mathrm{H} & 0.473895000 & 6.705509000 & 6.021587000 \\ \mathrm{C} & 1.921260000 & 5.281470000 & 5.280562000 \\ \mathrm{H} & 3.434308000 & 2.459205000 & 4.112219000 \\ \mathrm{~B} & 4.255048000 & 5.371533000 & 6.016923000 \\ & & & \end{array}$

Page S62 


$\begin{array}{llll}\text { O } & 4.766650000 & 6.407197000 & 5.083007000 \\ \text { O } & 5.031018000 & 5.446485000 & 7.218297000 \\ \text { C } & 5.944044000 & 7.024384000 & 5.697675000 \\ \text { C } & 5.689154000 & 6.748780000 & 7.231559000 \\ \text { C } & 4.724934000 & 7.770168000 & 7.859938000 \\ \text { C } & 6.963496000 & 6.635830000 & 8.069991000 \\ \text { C } & 5.966711000 & 8.503019000 & 5.306642000 \\ \mathrm{C} & 7.206344000 & 6.326170000 & 5.170977000 \\ \mathrm{H} & 6.162894000 & 8.596670000 & 4.223798000 \\ \mathrm{H} & 5.004357000 & 8.992928000 & 5.526327000 \\ \mathrm{H} & 7.206695000 & 6.372613000 & 4.068600000 \\ \mathrm{H} & 5.198838000 & 8.760956000 & 7.973719000 \\ \mathrm{H} & 3.811224000 & 7.878038000 & 7.250933000 \\ \mathrm{H} & 7.229301000 & 5.264027000 & 5.464580000 \\ \mathrm{H} & 8.124472000 & 6.814462000 & 5.540647000 \\ \mathrm{H} & 6.768382000 & 9.036030000 & 5.849109000 \\ \mathrm{H} & 4.430775000 & 7.411627000 & 8.862091000 \\ \mathrm{H} & 7.556623000 & 7.565552000 & 8.002734000 \\ \mathrm{H} & 7.589468000 & 5.792103000 & 7.736318000 \\ \mathrm{H} & 6.701694000 & 6.472797000 & 9.130600000 \\ \mathrm{H} & 4.462179000 & 3.647903000 & 2.341908000\end{array}$

\section{${ }^{1}$ 5I-A FSPE: -2877.255990868460 $E_{\mathrm{h}}$}

\begin{tabular}{|c|c|c|c|}
\hline & 0 & 0 & \\
\hline & .924856000 & 5.904680000 & 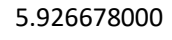 \\
\hline & 000 & 00 & סח \\
\hline & 63000 & 79000 & 000 \\
\hline & 979834000 & .529719000 & 000 \\
\hline & 8.037993000 & 5.268835000 & 24000 \\
\hline & 4.415380000 & 4.233916000 & 5000 \\
\hline & 9.343726000 & 5.260811000 & רחת 12 \\
\hline & 560000 & 66000 & .39786 \\
\hline & 278000 & 416000 & 3.193240000 \\
\hline & 11000 & & \\
\hline & 10.842262000 & 029000 & 0.12 \\
\hline & 9.012958000 & 7.505560000 & 0.412 \\
\hline & 9.3878920 & 8.3 & -0.1 \\
\hline & 7.727837000 & 7.541516000 & 0.954873000 \\
\hline & 7.087268000 & 1701000 & 0.84026300 \\
\hline & 7.198654000 & 03000 & 000 \\
\hline & 6.13 & 00 & 0 \\
\hline & 34000 & 980000 & 7000 \\
\hline & 5.513425000 & & 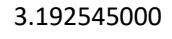 \\
\hline & 5.370626000 & 67000 & 00 \\
\hline & 4.810108000 & 78000 & .56 \\
\hline & 594000 & 58000 & 000 \\
\hline & 41000 & 25000 & 000 \\
\hline & 45000 & 23000 & 000 \\
\hline & 0.98 & 43000 & 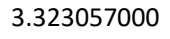 \\
\hline & 1.137544000 & & \\
\hline & -0.286091000 & & \\
\hline & -1.132687000 & 389207000 & 2.90122500 \\
\hline & 177000 & 328000 & 4.550728000 \\
\hline & 525000 & 344000 & 00 \\
\hline & 0.622580000 & 6.042180000 & 5.35372 \\
\hline & 0.500272000 & .789216000 & .147778000 \\
\hline & 1.904430000 & 5.487841000 & 5000 \\
\hline & 3.454554000 & 2.968262000 & 3.392222000 \\
\hline & 4.343875000 & 5.485195000 & 5.775592000 \\
\hline & 5.178871000 & 6.517512000 & 5.1235500 \\
\hline & 4.948575000 & 5.218199000 & 7.05854700 \\
\hline & 6.311676000 & 6.842698000 & 5.98093600 \\
\hline & 5.798333000 & 6.352048000 & 7.394631000 \\
\hline & 4.930356000 & 7.407442000 & 8.10366000 \\
\hline
\end{tabular}

Page $\mathbf{6 6 3}$ 


$\begin{array}{llll}\text { C } & 6.907812000 & 5.867411000 & 8.330203000 \\ \text { C } & 6.557185000 & 8.351086000 & 5.877057000 \\ \text { C } & 7.555753000 & 6.092376000 & 5.489600000 \\ \text { H } & 6.917061000 & 8.594092000 & 4.861671000 \\ \text { H } & 5.632405000 & 8.921656000 & 6.058702000 \\ \text { H } & 7.704510000 & 6.319635000 & 4.421087000 \\ \text { H } & 5.533461000 & 8.262459000 & 8.456589000 \\ \text { H } & 4.137025000 & 7.782387000 & 7.434750000 \\ \text { H } & 7.445102000 & 5.001789000 & 5.589103000 \\ \text { H } & 8.457385000 & 6.405838000 & 6.043967000 \\ \text { H } & 7.324951000 & 8.674221000 & 6.603286000 \\ \text { H } & 4.448359000 & 6.940041000 & 8.980434000 \\ \text { H } & 7.638909000 & 6.674389000 & 8.517699000 \\ \text { H } & 7.440894000 & 5.000654000 & 7.906035000 \\ \text { H } & 6.475409000 & 5.565221000 & 9.300741000 \\ \text { H } & 4.726009000 & 4.409321000 & 1.964701000 \\ \text { C } & 2.907059000 & 5.741817000 & 0.666670000 \\ \text { C } & 1.515977000 & 5.821167000 & 0.394892000 \\ \text { H } & 0.836625000 & 6.155458000 & 1.187612000 \\ \text { C } & 1.020579000 & 5.451074000 & -0.865382000 \\ \text { H } & -0.057554000 & 5.509475000 & -1.061983000 \\ \text { C } & 1.898323000 & 4.999981000 & -1.869867000 \\ \text { H } & 1.506748000 & 4.708052000 & -2.852463000 \\ \text { C } & 3.280801000 & 4.920786000 & -1.607964000 \\ \text { H } & 3.969128000 & 4.571510000 & -2.388081000 \\ \text { C } & 3.783641000 & 5.284706000 & -0.350564000 \\ \text { H } & 4.857282000 & 5.227055000 & -0.134037000 \\ \text { C } & 3.397204000 & 6.144733000 & 1.960897000 \\ \text { C } & 3.500690000 & 6.779809000 & 3.041977000 \\ \text { H } & 3.392174000 & 7.547454000 & 3.797079000\end{array}$

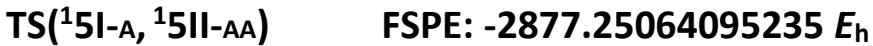

$\begin{array}{lrrr}\text { Fe } & 0.106814000 & -0.359643000 & -0.214542000 \\ \text { O } & -1.889127000 & 0.000059000 & 2.581899000 \\ \text { C } & 2.759859000 & -1.396098000 & -0.985025000 \\ \text { H } & 3.477976000 & -2.233353000 & -1.025856000 \\ \text { O } & 1.130454000 & 1.058304000 & -1.023222000 \\ \text { C } & 3.142971000 & -0.176389000 & -1.651943000 \\ \text { N } & -0.357851000 & -1.572356000 & 1.354073000 \\ \text { C } & 4.399854000 & -0.121291000 & -2.317171000 \\ \text { H } & 5.019659000 & -1.028604000 & -2.334215000 \\ \text { N } & 1.652215000 & -1.567389000 & -0.323571000 \\ \text { C } & 4.853996000 & 1.047396000 & -2.930125000 \\ \text { H } & 5.824188000 & 1.069643000 & -3.440321000 \\ \text { C } & 4.041334000 & 2.207466000 & -2.875729000 \\ \text { H } & 4.385082000 & 3.137626000 & -3.347718000 \\ \text { C } & 2.802279000 & 2.185929000 & -2.232669000 \\ \text { H } & 2.170063000 & 3.081623000 & -2.188823000 \\ \text { C } & 2.306749000 & 0.997240000 & -1.611203000 \\ \text { C } & 1.409034000 & -2.883330000 & 0.290844000 \\ \text { H } & 2.346559000 & -3.440232000 & 0.469079000 \\ \text { H } & 0.787002000 & -3.480916000 & -0.403280000 \\ \text { C } & 0.678223000 & -2.613311000 & 1.592924000 \\ \text { H } & 0.193377000 & -3.516290000 & 2.002816000 \\ \text { H } & 1.360841000 & -2.207499000 & 2.353854000 \\ \text { C } & -1.383327000 & -1.869418000 & 0.499117000 \\ \text { C } & -2.699082000 & -1.309247000 & 0.706616000 \\ \text { C } & -3.780060000 & -1.635906000 & -0.146170000 \\ \text { H } & -3.604997000 & -2.337388000 & -0.972071000 \\ \text { C } & -5.041805000 & -1.060521000 & 0.042163000 \\ \text { H } & -5.870431000 & -1.315401000 & -0.629040000 \\ \text { C } & -5.234276000 & -0.142618000 & 1.098270000 \\ \text { H } & -6.219710000 & 0.314908000 & 1.253924000 \\ \text { C } & -4.174415000 & 0.201343000 & 1.951206000\end{array}$




$\begin{array}{lrrr}\text { H } & -4.310383000 & 0.919776000 & 2.768854000 \\ \text { C } & -2.895538000 & -0.363483000 & 1.756566000 \\ \text { H } & -1.287941000 & -2.776100000 & -0.111808000 \\ \text { H } & -1.834252000 & 1.732497000 & -0.049493000 \\ \text { B } & -0.464841000 & -0.343833000 & 2.367594000 \\ \text { O } & 0.283156000 & 0.720873000 & 1.652640000 \\ \text { O } & 0.217789000 & -0.555324000 & 3.615554000 \\ \text { C } & 1.433828000 & 1.134273000 & 2.463246000 \\ \text { C } & 0.981598000 & 0.654311000 & 3.901068000 \\ \text { C } & 0.050921000 & 1.667792000 & 4.591177000 \\ \text { C } & 2.138098000 & 0.284627000 & 4.831503000 \\ \text { C } & 1.582403000 & 2.648485000 & 2.297907000 \\ \text { C } & 2.709344000 & 0.440728000 & 1.977416000 \\ \text { H } & 1.890989000 & 2.868565000 & 1.260799000 \\ \text { H } & 0.630695000 & 3.168675000 & 2.490558000 \\ \text { H } & 2.852819000 & 0.660783000 & 0.909291000 \\ \text { H } & 0.600214000 & 2.572764000 & 4.905117000 \\ \text { H } & -0.777114000 & 1.966261000 & 3.926366000 \\ \text { H } & 2.656466000 & -0.651894000 & 2.097263000 \\ \text { H } & 3.589114000 & 0.812635000 & 2.530077000 \\ \text { H } & 2.352031000 & 3.044075000 & 2.985287000 \\ \text { H } & -0.382920000 & 1.197133000 & 5.490898000 \\ \text { H } & 2.811810000 & 1.148132000 & 4.976806000 \\ \text { H } & 2.724524000 & -0.557523000 & 4.429304000 \\ \text { H } & 1.742511000 & -0.011071000 & 5.819416000 \\ \text { C } & -1.967578000 & -0.372589000 & -2.821283000 \\ \text { C } & -1.401144000 & -1.398471000 & -3.615912000 \\ \text { H } & -0.452570000 & -1.855451000 & -3.300550000 \\ \text { C } & -2.038218000 & -1.830568000 & -4.790054000 \\ \text { H } & -1.583747000 & -2.626432000 & -5.393520000 \\ \text { C } & -3.253848000 & -1.246158000 & -5.191401000 \\ \text { H } & -3.752868000 & -1.584186000 & -6.108181000 \\ \text { C } & -3.831040000 & -0.227761000 & -4.404239000 \\ \text { H } & -4.783601000 & 0.226360000 & -4.705987000 \\ \text { C } & -3.201376000 & 0.203708000 & -3.229710000 \\ \text { H } & -3.658330000 & 0.980302000 & -2.604482000 \\ \text { C } & -1.352907000 & 0.102219000 & -1.595947000 \\ \text { C } & -1.408706000 & 0.879387000 & -0.571398000 \\ \text { H } & -0.168280000 & -0.956942000 & -1.561039000\end{array}$

\section{$\mathrm{TS}\left({ }^{1} 5 \mathrm{I}-\mathrm{B},{ }^{1} 5 \mathrm{II-BC}\right) \quad$ FSPE: $-2877.25350659287 E_{\mathrm{h}}$}

$\begin{array}{lrrr}\text { Fe } & 0.064685000 & -0.408966000 & -0.290274000 \\ \text { O } & -1.942902000 & -0.102203000 & 2.566025000 \\ \text { C } & 2.782186000 & -1.328766000 & -0.955429000 \\ \text { H } & 3.527541000 & -2.142360000 & -0.972150000 \\ \text { O } & 1.050948000 & 1.042238000 & -1.088519000 \\ \text { C } & 3.152591000 & -0.090651000 & -1.592481000 \\ \text { N } & -0.374651000 & -1.597247000 & 1.283724000 \\ \text { C } & 4.442819000 & 0.023172000 & -2.182266000 \\ \text { H } & 5.100721000 & -0.856954000 & -2.169533000 \\ \text { N } & 1.655622000 & -1.544364000 & -0.339832000 \\ \text { C } & 4.880990000 & 1.216644000 & -2.757124000 \\ \text { H } & 5.877471000 & 1.285520000 & -3.209112000 \\ \text { C } & 4.018038000 & 2.341695000 & -2.739918000 \\ \text { H } & 4.350213000 & 3.290522000 & -3.181995000 \\ \text { C } & 2.745220000 & 2.261952000 & -2.172404000 \\ \text { H } & 2.073185000 & 3.129029000 & -2.157742000 \\ \text { C } & 2.264587000 & 1.045449000 & -1.594638000 \\ \text { C } & 1.426557000 & -2.876414000 & 0.246394000 \\ \text { H } & 2.372074000 & -3.415406000 & 0.436576000 \\ \text { H } & 0.832973000 & -3.472839000 & -0.473039000 \\ \text { C } & 0.659827000 & -2.638618000 & 1.533125000 \\ \text { H } & 0.169005000 & -3.551783000 & 1.912047000\end{array}$




\begin{tabular}{|c|c|c|c|}
\hline & & & \\
\hline & -1.399492000 & -1.89046 & \\
\hline & -2.743152000 & -1.419511000 & 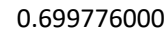 \\
\hline & -3.846772000 & -1.826930000 & 4000 \\
\hline & -3.662767000 & 9000 & 96000 \\
\hline & -5.149669000 & -1.43 & 51000 \\
\hline & -5.997470000 & 5000 & 6000 \\
\hline & 000 & & \\
\hline & 000 & & \\
\hline & -4.28 & & \\
\hline & -4.428885000 & 000 & 200 \\
\hline & -2.962165000 & -0.558607000 & 84000 \\
\hline & -1.2933 & -2.793 & 2000 \\
\hline & -1.6592 & -0.4 & \\
\hline & -0.51 & & \\
\hline & 0.19 & & \\
\hline & $0.1 \varepsilon$ & & \\
\hline & 1.33 & & \\
\hline & 0.8 & & \\
\hline & -0.1 & & \\
\hline & 2.0 & & \\
\hline & 1.47 & & \\
\hline & 2.62 & & \\
\hline & 1.81 & & \\
\hline & & & \\
\hline & & & \\
\hline & & & 000 \\
\hline & -0.9 & & 000 \\
\hline & 2.5 & & \\
\hline & 3.4 & & \\
\hline & 2.2 & & \\
\hline & -0.5 & & \\
\hline & 2.66 & & \\
\hline & 2.659059000 & & \\
\hline & & & \\
\hline & -2.1 & & -0. \\
\hline & -3.5 & & \\
\hline & -4.15 & 000 & -0 \\
\hline & -4.0 & & \\
\hline & -5.1 & & \\
\hline & -3.25 & 4.25 & \\
\hline & -3.691 & & \\
\hline & -1.855361000 & 4.170449000 & 0.9 \\
\hline & -1.2050 & 2000 & 1000 \\
\hline & -1.2 & 5000 & \\
\hline & & & 00 \\
\hline & & & -1 \\
\hline & & & \\
\hline & & & \\
\hline
\end{tabular}

\section{${ }^{1}$ 5II-AA FSPE: - 2877.28270273802 $E_{\mathrm{h}}$}

$\begin{array}{lrrr}\mathrm{Fe} & 0.212029000 & 0.208065000 & 0.677975000 \\ \mathrm{O} & -1.632404000 & 1.634568000 & 3.262416000 \\ \mathrm{C} & 1.907271000 & -1.996311000 & -0.266591000 \\ \mathrm{H} & 2.206059000 & -3.058259000 & -0.220794000 \\ \mathrm{O} & 1.368207000 & 0.862301000 & -0.644741000 \\ \mathrm{C} & 2.555979000 & -1.183976000 & -1.248299000 \\ \mathrm{~N} & -0.551611000 & -0.452058000 & 2.368405000 \\ \mathrm{C} & 3.543910000 & -1.771012000 & -2.096238000 \\ \mathrm{H} & 3.738799000 & -2.847939000 & -2.003481000 \\ \mathrm{~N} & 1.032305000 & -1.554006000 & 0.609897000 \\ \mathrm{C} & 4.256262000 & -1.004381000 & -3.012289000 \\ \mathrm{H} & 5.012052000 & -1.468982000 & -3.656447000\end{array}$


$\begin{array}{llll}\text { C } & 4.002148000 & 0.392000000 & -3.099617000\end{array}$

$\begin{array}{llll}\text { H } & 4.565587000 & 1.002980000 & -3.816715000\end{array}$

$\begin{array}{llll}\text { C } & 3.045262000 & 0.996542000 & -2.288028000\end{array}$

H $\quad 2.839242000 \quad 2.071993000 \quad-2.349328000$

$\begin{array}{llll}\text { C } & 2.282211000 & 0.229439000 & -1.354823000\end{array}$

C $\quad 0.533819000 \quad-2.510441000 \quad 1.625708000$

H $\quad 1.309122000 \quad-3.246419000 \quad 1.901105000$

$\begin{array}{llll}H & -0.320629000 & -3.072043000 & 1.201927000\end{array}$

C $\quad 0.118132000 \quad-1.685907000 \quad 2.838440000$

H $\quad-0.564174000 \quad-2.245652000 \quad 3.503707000$

$\begin{array}{llll}\text { H } & 0.994592000 & -1.369043000 & 3.424397000\end{array}$

$\begin{array}{llll}\text { C } & -1.590972000 & -0.564636000 & 1.451733000\end{array}$

$\begin{array}{llll}\text { C } & -2.768404000 & 0.283362000 & 1.599119000\end{array}$

$\begin{array}{llll}\text { C } & -3.938877000 & 0.056484000 & 0.840387000\end{array}$

$\begin{array}{llll}\text { H } & -3.954673000 & -0.786129000 & 0.136155000\end{array}$

C $\quad-5.058860000 \quad 0.889326000 \quad 0.975690000$

$\begin{array}{llll}\text { H } & -5.959562000 & 0.704703000 & 0.378058000\end{array}$

$\begin{array}{llll}\text { C } & -5.012029000 & 1.969845000 & 1.880977000\end{array}$

H $\quad-5.879267000 \quad 2.633516000 \quad 1.989217000$

$\begin{array}{llll}\text { C } & -3.859931000 & 2.210220000 & 2.646248000\end{array}$

$\begin{array}{llll}\text { H } & -3.809286000 & 3.049484000 & 3.350802000\end{array}$

$\begin{array}{llll}\text { C } & -2.731103000 & 1.378478000 & 2.507469000\end{array}$

H $\quad-1.758413000 \quad-1.556006000 \quad 1.007598000$

$\begin{array}{llll}H & -0.330344000 & 3.310617000 & 0.629625000\end{array}$

$\begin{array}{llll}\text { B } & -0.347391000 & 0.955796000 & 3.062561000\end{array}$

$\begin{array}{llll}\text { O } & 0.504060000 & 1.653655000 & 2.011641000\end{array}$

$\begin{array}{llll}\text { O } & 0.448137000 & 0.931212000 & 4.253321000\end{array}$

C $\quad 1.842607000 \quad 1.906297000 \quad 2.597543000$

C $\quad 1.459947000 \quad 1.975701000 \quad 4.127961000$

C $\quad 0.831248000 \quad 3.328474000 \quad 4.506584000$

$\begin{array}{llll}\text { C } & 2.610718000 & 1.642162000 & 5.077896000\end{array}$

$\begin{array}{llll}\text { C } & 2.349844000 & 3.206327000 & 1.976814000\end{array}$

$\begin{array}{llll}\text { C } & 2.825863000 & 0.768831000 & 2.299678000\end{array}$

$\begin{array}{llll}\text { H } & 2.538243000 & 3.039234000 & 0.901879000\end{array}$

$\begin{array}{llll}\text { H } & 1.610534000 & 4.016805000 & 2.075198000\end{array}$

$\begin{array}{llll}\text { H } & 2.945696000 & 0.644839000 & 1.213218000\end{array}$

$\begin{array}{llll}\text { H } & 1.587604000 & 4.132760000 & 4.518617000\end{array}$

$\begin{array}{llll}H & 0.026568000 & 3.605263000 & 3.804720000\end{array}$

H $\quad 2.492781000 \quad-0.189792000 \quad 2.725155000$

$\begin{array}{llll}H & 3.814542000 & 1.014838000 & 2.722809000\end{array}$

$\begin{array}{llll}\text { H } & 3.293448000 & 3.522477000 & 2.456542000\end{array}$

$\begin{array}{llll}H & 0.395984000 & 3.248079000 & 5.517886000\end{array}$

$\begin{array}{llll}H & 3.461893000 & 2.327163000 & 4.914950000\end{array}$

$\begin{array}{llll}\text { H } & 2.957729000 & 0.605221000 & 4.940770000\end{array}$

$\begin{array}{llll}\text { H } & 2.273214000 & 1.757675000 & 6.123041000\end{array}$

$\begin{array}{llll}\text { C } & -2.023679000 & 3.726646000 & -0.693652000\end{array}$

C $\quad-3.282923000 \quad 3.277135000 \quad-1.174217000$

H $\quad-3.572835000 \quad 2.231954000 \quad-1.010561000$

C $\quad-4.177727000 \quad 4.159024000 \quad-1.798467000$

H $\quad-5.148231000 \quad 3.785455000-2.151771000$

C $\quad-3.850321000 \quad 5.521932000 \quad-1.952444000$

H $\quad-4.554743000 \quad 6.211554000 \quad-2.434992000$

$\begin{array}{llll}\text { C } & -2.616795000 & 5.991010000 & -1.461797000\end{array}$

H $\quad-2.351962000 \quad 7.051935000 \quad-1.563895000$

$\begin{array}{llll}\text { C } & -1.719898000 & 5.106319000 & -0.838740000\end{array}$

$\begin{array}{llll}\text { H } & -0.759615000 & 5.482679000 & -0.458834000\end{array}$

$\begin{array}{llll}\text { C } & -1.061465000 & 2.824973000 & -0.032850000\end{array}$

$\begin{array}{llll}\text { C } & -1.017903000 & 1.477985000 & -0.151223000\end{array}$

H $\quad-1.717979000 \quad 0.968830000 \quad-0.841305000$

\section{${ }^{1}{ }_{5 \mathrm{II}-\mathrm{AB}}$ FSPE: -2877.30692680372 $E_{\mathrm{h}}$}

Fe $\quad 4.816734000 \quad 4.680724000 \quad 3.178039000$

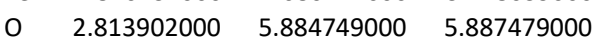


$\begin{array}{llll}\text { C } \quad 7.450655000 & 3.532150000 & 2.774566000\end{array}$

$\begin{array}{llll}\text { H } & 8.188199000 & 2.751622000 & 3.018791000\end{array}$

$\begin{array}{llll}\text { O } & 5.751189000 & 5.776689000 & 1.933747000\end{array}$

$\begin{array}{llll}\text { C } & 7.817276000 & 4.487493000 & 1.756437000\end{array}$

$\begin{array}{lllll}\text { N } & 4.328574000 & 4.025584000 & 5.075532000\end{array}$

$\begin{array}{llll}\text { C } & 9.090040000 & 4.373552000 & 1.128842000\end{array}$

$\begin{array}{llll}\text { H } & 9.741515000 & 3.538527000 & 1.419735000\end{array}$

N $\quad 6.326281000 \quad 3.537745000 \quad 3.449531000$

$\begin{array}{llll}\text { C } & 9.515720000 & 5.293290000 & 0.170126000\end{array}$

$\begin{array}{llll}\text { H } & 10.498085000 & 5.187081000 & -0.304633000\end{array}$

$\begin{array}{llll}\text { C } & 8.662396000 & 6.370384000 & -0.177624000\end{array}$

H $\quad 8.986048000 \quad 7.102781000 \quad-0.928681000$

$\begin{array}{llll}\text { C } & 7.408620000 & 6.512995000 & 0.420175000\end{array}$

$\begin{array}{llll}\text { H } & 6.744238000 & 7.344516000 & 0.154576000\end{array}$

$\begin{array}{llll}\text { C } & 6.944945000 & 5.577631000 & 1.392249000\end{array}$

$\begin{array}{llll}\text { C } & 6.080272000 & 2.436647000 & 4.417334000\end{array}$

$\begin{array}{llll}\text { H } & 7.019122000 & 1.951535000 & 4.735829000\end{array}$

$\begin{array}{llll}H & 5.459786000 & 1.674138000 & 3.907727000\end{array}$

C $\quad 5.345697000 \quad 3.057709000 \quad 5.598386000$

$\begin{array}{llll}\text { H } & 4.841428000 & 2.307390000 & 6.231131000\end{array}$

$\begin{array}{llll}\text { H } & 6.035197000 & 3.638176000 & 6.228040000\end{array}$

$\begin{array}{llll}\text { C } & 3.272623000 & 3.512471000 & 4.343079000\end{array}$

$\begin{array}{llll}\text { C } & 1.960955000 & 4.125203000 & 4.432606000\end{array}$

C $\quad 0.844474000 \quad 3.571799000 \quad 3.756550000$

$\begin{array}{llll}\text { H } & 0.988728000 & 2.660548000 & 3.162239000\end{array}$

$\begin{array}{llll}\text { C } & -0.417409000 & 4.170179000 & 3.844972000\end{array}$

H $\quad-1.273597000 \quad 3.733630000 \quad 3.317319000$

$\begin{array}{llll}\text { C } & -0.579181000 & 5.335362000 & 4.628283000\end{array}$

$\begin{array}{llll}\text { H } & -1.565576000 & 5.809755000 & 4.707003000\end{array}$

$\begin{array}{llll}\text { C } & 0.508622000 & 5.895970000 & 5.313970000\end{array}$

$\begin{array}{llll}\text { H } & 0.391474000 & 6.799647000 & 5.924426000\end{array}$

$\begin{array}{llll}\text { C } & 1.791197000 & 5.312073000 & 5.215566000\end{array}$

$\begin{array}{llll}\text { H } & 3.331973000 & 2.461082000 & 4.036570000\end{array}$

$\begin{array}{llll}\text { H } & 2.302414000 & 4.044750000 & 1.423390000\end{array}$

$\begin{array}{llll}\text { B } \quad 4.243536000 & 5.489101000 & 5.776598000\end{array}$

$\begin{array}{llll}\text { O } & 4.998985000 & 6.396958000 & 4.873766000\end{array}$

$\begin{array}{llll}0 & 4.903107000 & 5.543950000 & 7.055581000\end{array}$

$\begin{array}{llll}\text { C } & 6.134364000 & 6.970516000 & 5.594504000\end{array}$

$\begin{array}{llll}\text { C } & 5.690942000 & 6.771143000 & 7.100136000\end{array}$

$\begin{array}{llll}\text { C } & 4.779721000 & 7.906711000 & 7.600558000\end{array}$

$\begin{array}{llll}\text { C } & 6.854730000 & 6.563441000 & 8.070886000\end{array}$

C $\quad 6.282272000 \quad 8.429892000 \quad 5.153555000$

$\begin{array}{llll}\text { C } & 7.409518000 & 6.199234000 & 5.232691000\end{array}$

$\begin{array}{llll}\text { H } & 6.595875000 & 8.460942000 & 4.095043000\end{array}$

$\begin{array}{llll}H & 5.330343000 & 8.977068000 & 5.245791000\end{array}$

$\begin{array}{llll}H & 7.524823000 & 6.200114000 & 4.136709000\end{array}$

$\begin{array}{llll}\text { H } & 5.338677000 & 8.849549000 & 7.733469000\end{array}$

$\begin{array}{llll}H & 3.944185000 & 8.083201000 & 6.901987000\end{array}$

H $\quad 7.363442000 \quad 5.151304000 \quad 5.566485000$

$\begin{array}{llll}H & 8.300816000 & 6.671993000 & 5.679098000\end{array}$

$\begin{array}{llll}\text { H } & 7.050131000 & 8.945258000 & 5.757984000\end{array}$

$\begin{array}{llll}\text { H } & 4.354980000 & 7.616473000 & 8.577523000\end{array}$

$\begin{array}{llll}\text { H } & 7.539403000 & 7.430239000 & 8.048388000\end{array}$

$\begin{array}{llll}\text { H } & 7.427464000 & 5.654824000 & 7.822936000\end{array}$

$\begin{array}{llll}\text { H } & 6.469224000 & 6.458150000 & 9.100457000\end{array}$

$\begin{array}{llll}\text { C } & 2.804585000 & 2.576522000 & -0.096648000\end{array}$

C $\quad 3.761807000 \quad 1.806625000 \quad-0.811481000$

H $\quad 4.817941000 \quad 1.842889000 \quad-0.514408000$

$\begin{array}{llll}\text { C } & 3.382873000 & 1.006698000 & -1.899465000\end{array}$

H $\quad 4.145045000 \quad 0.424037000 \quad-2.433582000$

$\begin{array}{llll}\text { C } & 2.036425000 & 0.951566000 & -2.315263000\end{array}$

$\begin{array}{llll}\text { H } & 1.744091000 & 0.326925000 & -3.168757000\end{array}$

C $\quad 1.074204000 \quad 1.711983000 \quad-1.624764000$

H $\quad 0.021901000 \quad 1.682858000 \quad-1.936777000$

$\begin{array}{llll}\text { C } & 1.454464000 & 2.512080000 & -0.534487000\end{array}$

$\begin{array}{llll}\text { H } & 0.694755000 & 3.099949000 & -0.000471000\end{array}$

Page S68 
$\begin{array}{llll}\text { C } & 3.140299000 & 3.424871000 & 1.063063000\end{array}$

$\begin{array}{llll}\text { C } & 4.328646000 & 3.486745000 & 1.725942000\end{array}$

$\begin{array}{llll}\text { H } & 5.160457000 & 2.843642000 & 1.376791000\end{array}$

\section{${ }^{1}$ 5II-BC FSPE: -2877.30734091867 $E_{\mathrm{h}}$}

$\begin{array}{llll}\text { Fe } & -0.022937000 & 0.217844000 & 0.878883000\end{array}$

$\begin{array}{llll}\text { O } & -1.447643000 & 1.614240000 & 3.798158000\end{array}$

C $\quad 1.841004000 \quad-1.628961000 \quad-0.125154000$

H $\quad 2.301842000 \quad-2.627286000 \quad-0.073428000$

$\begin{array}{llll}\text { O } & 1.055637000 & 1.176973000 & -0.285075000\end{array}$

C $\quad 2.332634000 \quad-0.712903000 \quad-1.124706000$

$\begin{array}{llll}\text { N } & -0.635085000 & -0.467915000 & 2.643982000\end{array}$

C $\quad 3.298643000 \quad-1.162780000 \quad-2.066223000$

H $\quad 3.631518000 \quad-2.208381000 \quad-2.015374000$

$\begin{array}{llll}\mathrm{N} & 0.881450000 & -1.361775000 & 0.727327000\end{array}$

C $\quad 3.817476000 \quad-0.310097000 \quad-3.044867000$

H $\quad 4.555512000 \quad-0.679863000 \quad-3.766654000$

$\begin{array}{llll}\text { C } & 3.381343000 & 1.035158000 & -3.090860000\end{array}$

H $\quad 3.780207000 \quad 1.714467000 \quad-3.855540000$

$\begin{array}{llll}\text { C } & 2.442895000 & 1.512660000 & -2.168998000\end{array}$

$\begin{array}{llll}\text { H } & 2.102788000 & 2.555321000 & -2.191224000\end{array}$

C $\quad 1.895445000 \quad 0.659331000 \quad-1.173228000$

$\begin{array}{llll}\text { C } & 0.400481000 & -2.429145000 & 1.630660000\end{array}$

H $\quad 1.168493000 \quad-3.207828000 \quad 1.779257000$

H $\quad-0.476915000 \quad-2.902048000 \quad 1.149003000$

C $\quad 0.025406000 \quad-1.760593000 \quad 2.945706000$

H $\quad-0.645765000 \quad-2.390128000 \quad 3.556037000$

$\begin{array}{llll}\text { H } & 0.922678000 & -1.522523000 & 3.537769000\end{array}$

$\begin{array}{llll}\text { C } & -1.763255000 & -0.451576000 & 1.843618000\end{array}$

$\begin{array}{llll}\text { C } & -2.773876000 & 0.579977000 & 2.051815000\end{array}$

$\begin{array}{llll}\text { C } & -3.980269000 & 0.576530000 & 1.311759000\end{array}$

$\begin{array}{llll}\text { H } & -4.143430000 & -0.222914000 & 0.579409000\end{array}$

$\begin{array}{llll}\text { C } & -4.930872000 & 1.586911000 & 1.481539000\end{array}$

$\begin{array}{llll}\text { H } & -5.847090000 & 1.586022000 & 0.880002000\end{array}$

$\begin{array}{llll}\text { C } & -4.695099000 & 2.612611000 & 2.423099000\end{array}$

$\begin{array}{llll}\text { H } & -5.433109000 & 3.413485000 & 2.558498000\end{array}$

$\begin{array}{llll}\text { C } & -3.524459000 & 2.616170000 & 3.194371000\end{array}$

$\begin{array}{llll}\text { H } & -3.333803000 & 3.396901000 & 3.940940000\end{array}$

$\begin{array}{llll}\text { C } & -2.555331000 & 1.606745000 & 3.013528000\end{array}$

H $\quad-2.101982000 \quad-1.411995000 \quad 1.434960000$

$\begin{array}{llll}\text { B } & -0.235382000 & 0.863154000 & 3.425492000\end{array}$

$\begin{array}{llll}\text { O } & 0.557360000 & 1.591974000 & 2.383496000\end{array}$

$\begin{array}{llll}\text { O } & 0.635120000 & 0.662592000 & 4.543385000\end{array}$

$\begin{array}{llll}\text { C } & 1.913796000 & 1.834378000 & 2.917960000\end{array}$

C $\quad 1.663319000 \quad 1.698391000 \quad 4.472009000$

C $\quad 1.112112000 \quad 2.994096000 \quad 5.093237000$

C $\quad 2.879656000 \quad 1.214912000 \quad 5.264014000$

$\begin{array}{llll}\text { C } & 2.359011000 & 3.216181000 & 2.442617000\end{array}$

$\begin{array}{llll}\text { C } & 2.868500000 & 0.758510000 & 2.388977000\end{array}$

$\begin{array}{llll}\text { H } & 2.491933000 & 3.194976000 & 1.346934000\end{array}$

$\begin{array}{llll}H & 1.610524000 & 3.987370000 & 2.685473000\end{array}$

$\begin{array}{llll}\text { H } & 2.869159000 & 0.777121000 & 1.289166000\end{array}$

$\begin{array}{llll}\text { H } & 1.891372000 & 3.774501000 & 5.147050000\end{array}$

$\begin{array}{llll}H & 0.255982000 & 3.383878000 & 4.518012000\end{array}$

$\begin{array}{llll}H & 2.559746000 & -0.247152000 & 2.716750000\end{array}$

$\begin{array}{llll}H & 3.897818000 & 0.944776000 & 2.739512000\end{array}$

$\begin{array}{llll}H & 3.321354000 & 3.494928000 & 2.908667000\end{array}$

$\begin{array}{llll}H & 0.767298000 & 2.778625000 & 6.119710000\end{array}$

H $\quad 3.732747000 \quad 1.90324000005 .126486000$

$\begin{array}{llll}H & 3.186277000 & 0.203161000 & 4.952866000\end{array}$

$\begin{array}{llll}\text { H } & 2.634057000 & 1.185097000 & 6.340295000\end{array}$

$\begin{array}{llll}\text { C } & -1.977716000 & 1.028113000 & -1.025097000\end{array}$

$\begin{array}{llll}\text { C } & -3.131446000 & 0.899374000 & -1.845086000\end{array}$ 


$\begin{array}{llll}\text { H } & -3.374378000 & -0.077191000 & -2.281330000 \\ \text { C } & -3.995060000 & 1.980061000 & -2.073699000 \\ \text { H } & -4.879698000 & 1.841434000 & -2.709038000 \\ \text { C } & -3.751757000 & 3.226201000 & -1.465855000 \\ \text { H } & -4.440897000 & 4.065405000 & -1.623605000 \\ \text { C } & -2.623925000 & 3.376375000 & -0.641423000 \\ \text { H } & -2.424109000 & 4.336262000 & -0.148846000 \\ \text { C } & -1.746671000 & 2.299657000 & -0.435003000 \\ \text { H } & -0.872029000 & 2.469294000 & 0.204467000 \\ \text { C } & -1.117682000 & -0.148229000 & -0.712661000 \\ \text { C } & -1.098498000 & -1.263738000 & -1.479025000 \\ \text { H } & -1.665363000 & -1.331980000 & -2.420447000 \\ \text { H } & -0.502758000 & -2.146201000 & -1.221051000\end{array}$

\section{${ }^{1}$ 5III-AAA $\quad$ FSPE: $-3185.70855058398 E_{\mathrm{h}}$}

$\begin{array}{lrrr}\text { Fe } & -0.203857000 & -0.182830000 & 0.285163000 \\ \text { O } & -1.708613000 & 1.580167000 & 2.892531000 \\ \text { C } & 2.289708000 & -1.834758000 & 0.102487000 \\ \text { H } & 2.883093000 & -2.709395000 & 0.420397000 \\ \text { O } & 0.974022000 & 0.578318000 & -1.021825000 \\ \text { C } & 2.854200000 & -0.999192000 & -0.927304000 \\ \text { N } & -0.725666000 & -0.643393000 & 2.238100000 \\ \text { C } & 4.111579000 & -1.357785000 & -1.481840000 \\ \text { H } & 4.604935000 & -2.263187000 & -1.102844000 \\ \text { N } & 1.144999000 & -1.613395000 & 0.675157000 \\ \text { C } & 4.711965000 & -0.601369000 & -2.488987000 \\ \text { H } & 5.678302000 & -0.900188000 & -2.911904000 \\ \text { C } & 4.047920000 & 0.559570000 & -2.955446000 \\ \text { H } & 4.505955000 & 1.171179000 & -3.744445000 \\ \text { C } & 2.809430000 & 0.936669000 & -2.433499000 \\ \text { H } & 2.284765000 & 1.827157000 & -2.801408000 \\ \text { C } & 2.159250000 & 0.164257000 & -1.421894000 \\ \text { C } & 0.652489000 & -2.578300000 & 1.661000000 \\ \text { H } & 1.440973000 & -3.269084000 & 2.013164000 \\ \text { H } & -0.141067000 & -3.185854000 & 1.181296000 \\ \text { C } & 0.087554000 & -1.756442000 & 2.802602000 \\ \text { H } & -0.541300000 & -2.350226000 & 3.488036000 \\ \text { H } & 0.896060000 & -1.287499000 & 3.382735000 \\ \text { C } & -1.909441000 & -0.948834000 & 1.633306000 \\ \text { C } & -3.047013000 & -0.068785000 & 1.732430000 \\ \text { C } & -4.301106000 & -0.416298000 & 1.172849000 \\ \text { H } & -4.409901000 & -1.394628000 & 0.686082000 \\ \text { C } & -5.383727000 & 0.469461000 & 1.239964000 \\ \text { H } & -6.351065000 & 0.193975000 & 0.803608000 \\ \text { C } & -5.219064000 & 1.718554000 & 1.880136000 \\ \text { H } & -6.060699000 & 2.420658000 & 1.932762000 \\ \text { C } & -3.986849000 & 2.081420000 & 2.442619000 \\ \text { H } & -3.845122000 & 3.056321000 & 2.923589000 \\ \text { C } & -2.884781000 & 1.203164000 & 2.363115000 \\ \text { H } & -2.081138000 & -2.002622000 & 1.378225000 \\ \text { H } & 0.117469000 & 2.745767000 & -0.529043000 \\ \text { B } & -0.433248000 & 0.833802000 & 2.756652000 \\ \text { O } & 0.456828000 & 1.344664000 & 1.691067000 \\ \text { O } & 0.315878000 & 0.912308000 & 3.984073000 \\ \text { C } & 1.722899000 & 1.792389000 & 2.280565000 \\ \text { C } & 1.303346000 & 1.969586000 & 3.797147000 \\ \text { C } & 0.633012000 & 3.330872000 & 4.060407000 \\ \text { C } & 2.439078000 & 1.744872000 & 4.796806000 \\ \text { C } & 2.126637000 & 3.082204000 & 1.560986000 \\ \text { H } & 2.818688000 & 0.742566000 & 2.098427000 \\ \text { H } & 2.399096000 & 2.842457000 & 0.517833000 \\ & 1.295321000 & 3.804039000 & 1.537034000\end{array}$




\begin{tabular}{|c|c|c|c|}
\hline & 000 & 7000 & \\
\hline & .368888000 & 153179000 & 8 \\
\hline & 166157000 & 50000 & 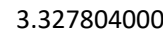 \\
\hline & .568966000 & 18000 & 00 \\
\hline & 3.777423000 & 113333000 & 00 \\
\hline & 2.999241000 & 3.548584000 & 2.0 \\
\hline & 0.181828000 & 3.317754000 & 5.0 \\
\hline & 3.270720000 & 2.446702000 & 4.60638000 \\
\hline & .825387000 & 4000 & 4. \\
\hline & 2.070382000 & 0000 & \\
\hline & 1000 & 1000 & -0.7 \\
\hline & -3.217260000 & 3.761841000 & -0.6 \\
\hline & -3.732005000 & 2.847463000 & -0.3 \\
\hline & -3.966371000 & 4.92 & -0.8 \\
\hline & -5.057794000 & 4.896369000 & -0.7 \\
\hline & -3.330243000 & 58000 & -1.2 \\
\hline & -3.919449000 & 7.03 & -1. \\
\hline & -1.9 & 6.14 & -1.3 \\
\hline & -1.4 & 000 & -1 . \\
\hline & -1.1 & & \\
\hline & -0.0 & 5.00586 & -1. \\
\hline & -0.966714000 & 2.568690000 & -0.5 \\
\hline & -1.406188000 & 1.316911000 & -0.2 \\
\hline & -2.498897000 & 1.169112000 & -0.2 \\
\hline & 0.356601000 & -2.46 & -2.1 \\
\hline & 0.8209680 & -3.72 & -1.7 \\
\hline & 000 & -4.17 & -0.8 \\
\hline & 1.855238000 & -4.382494000 & -2.416130000 \\
\hline & 2.217152000 & -5.350766000 & -2.0 \\
\hline & 2.435239000 & -3.798392000 & -3.5581560 \\
\hline & 3.255414000 & -4.309247000 & -4.07759 \\
\hline & 1.964348000 & -2.558583000 & 45220 \\
\hline & 2.420758000 & -2.098586000 & -4.9189890 \\
\hline & 0.935479000 & -1.892338000 & -3.36053400 \\
\hline & 0.589542000 & -0.909328000 & -3.6981870 \\
\hline & -0.616291000 & -1.719502000 & $-1.466982 C$ \\
\hline & -1.548920000 & -1.001025 & -1.007413 \\
\hline & -2.616838000 & -0.792162 & -1.071618 \\
\hline
\end{tabular}

\section{${ }^{1}$ 5III-AAB $\quad$ FSPE: $-3185.70642702484 E_{\mathrm{h}}$}

$\begin{array}{lrrr}\text { Fe } & -0.224479000 & -0.242565000 & 0.347374000 \\ \text { O } & -1.620551000 & 1.660479000 & 2.959351000 \\ \text { C } & 2.282850000 & -1.835681000 & 0.144873000 \\ \text { H } & 2.907899000 & -2.685959000 & 0.469882000 \\ \text { O } & 0.851274000 & 0.475784000 & -1.047168000 \\ \text { C } & 2.757045000 & -1.066449000 & -0.976119000 \\ \text { N } & -0.702515000 & -0.617550000 & 2.401533000 \\ \text { C } & 4.011827000 & -1.408551000 & -1.557394000 \\ \text { H } & 4.555750000 & -2.270375000 & -1.146248000 \\ \text { N } & 1.192945000 & -1.580609000 & 0.805106000 \\ \text { C } & 4.555139000 & -0.676888000 & -2.612010000 \\ \text { H } & 5.522171000 & -0.956297000 & -3.046438000 \\ \text { C } & 3.836036000 & 0.440629000 & -3.110143000 \\ \text { H } & 4.251476000 & 1.032086000 & -3.937017000 \\ \text { C } & 2.602718000 & 0.799668000 & -2.567937000 \\ \text { H } & 2.041922000 & 1.661917000 & -2.949598000 \\ \text { C } & 2.014996000 & 0.058895000 & -1.492448000 \\ \text { C } & 0.774983000 & -2.513692000 & 1.856919000 \\ \text { H } & 1.615383000 & -3.128789000 & 2.228691000 \\ \text { H } & 0.017616000 & -3.199307000 & 1.427516000 \\ \text { C } & 0.177683000 & -1.679923000 & 2.971549000 \\ \text { H } & -0.405125000 & -2.287924000 & 3.683805000 \\ \text { H } & 0.966310000 & -1.151486000 & 3.527418000\end{array}$


$\begin{array}{llll}\text { C } & -1.936065000 & -0.960445000 & 1.948503000\end{array}$

$\begin{array}{llll}\text { C } & -3.043526000 & -0.056992000 & 2.020069000\end{array}$

$\begin{array}{llll}\text { C } & -4.329525000 & -0.417650000 & 1.539383000\end{array}$

H $\quad-4.476711000 \quad-1.424682000 \quad 1.132698000$

$\begin{array}{llll}\text { C } & -5.382182000 & 0.499396000 & 1.561897000\end{array}$

$\begin{array}{llll}\text { H } & -6.367394000 & 0.217225000 & 1.173248000\end{array}$

$\begin{array}{llll}\text { C } & -5.163168000 & 1.796431000 & 2.083912000\end{array}$

$\begin{array}{llll}\text { H } & -5.983906000 & 2.524654000 & 2.096134000\end{array}$

$\begin{array}{llll}\text { C } & -3.905347000 & 2.175393000 & 2.570403000\end{array}$

$\begin{array}{llll}\text { H } & -3.721142000 & 3.186878000 & 2.950105000\end{array}$

$\begin{array}{llll}\text { C } & -2.826565000 & 1.265089000 & 2.528132000\end{array}$

$\begin{array}{llll}\text { H } & -2.111629000 & -2.016292000 & 1.705771000\end{array}$

$\begin{array}{llll}H & 0.045998000 & 2.663394000 & -0.651198000\end{array}$

$\begin{array}{llll}\text { B } & -0.358034000 & 0.882171000 & 2.823843000\end{array}$

$\begin{array}{llll}\text { O } & 0.512263000 & 1.317558000 & 1.718657000\end{array}$

$\begin{array}{llll}\text { O } & 0.422760000 & 1.017356000 & 4.028692000\end{array}$

$\begin{array}{llll}\text { C } & 1.776052000 & 1.831696000 & 2.243508000\end{array}$

$\begin{array}{llll}\text { C } & 1.407597000 & 2.061300000 & 3.768613000\end{array}$

$\begin{array}{llll}\text { C } & 0.750913000 & 3.433301000 & 4.010417000\end{array}$

$\begin{array}{llll}\text { C } & 2.576753000 & 1.866112000 & 4.735917000\end{array}$

$\begin{array}{llll}\text { C } & 2.112188000 & 3.103354000 & 1.457874000\end{array}$

$\begin{array}{llll}\text { C } & 2.895618000 & 0.806876000 & 2.053866000\end{array}$

$\begin{array}{llll}\text { H } & 2.351127000 & 2.829092000 & 0.415055000\end{array}$

$\begin{array}{llll}H & 1.257393000 & 3.797244000 & 1.436633000\end{array}$

$\begin{array}{llll}\text { H } & 2.991873000 & 0.571748000 & 0.983505000\end{array}$

H $\quad 1.485566000 \quad 4.251929000 \quad 3.913542000$

$\begin{array}{llll}\text { H } & -0.077209000 & 3.610941000 & 3.304647000\end{array}$

$\begin{array}{llll}H & 2.694009000 & -0.128925000 & 2.597701000\end{array}$

$\begin{array}{llll}H & 3.859491000 & 1.217960000 & 2.400385000\end{array}$

$\begin{array}{llll}\text { H } & 2.988088000 & 3.616045000 & 1.895143000\end{array}$

$\begin{array}{llll}H & 0.340822000 & 3.456295000 & 5.035476000\end{array}$

$\begin{array}{llll}\text { H } & 3.401184000 & 2.561767000 & 4.497442000\end{array}$

$\begin{array}{llll}H & 2.961931000 & 0.834087000 & 4.698495000\end{array}$

$\begin{array}{llll}\text { H } & 2.242746000 & 2.072587000 & 5.768345000\end{array}$

$\begin{array}{llll}\text { C } & -1.893952000 & 3.668478000 & -0.649575000\end{array}$

$\begin{array}{llll}\text { C } & -3.313844000 & 3.608931000 & -0.563765000\end{array}$

$\begin{array}{llll}\text { H } & -3.802766000 & 2.647805000 & -0.365145000\end{array}$

$\begin{array}{llll}\text { C } & -4.100767000 & 4.757736000 & -0.725896000\end{array}$

H $\quad-5.193608000 \quad 4.677823000 \quad-0.651114000$

$\begin{array}{llll}\text { C } & -3.502503000 & 6.008907000 & -0.983660000\end{array}$

H $\quad-4.121806000 \quad 6.906083000 \quad-1.110733000$

$\begin{array}{llll}\text { C } & -2.099722000 & 6.089758000 & -1.078244000\end{array}$

H $\quad-1.617160000 \quad 7.055485000 \quad-1.280211000$

C $\quad-1.311438000 \quad 4.938349000 \quad-0.915047000$

H $\quad-0.217222000 \quad 5.012497000 \quad-0.988470000$

$\begin{array}{llll}\text { C } & -1.024782000 & 2.495681000 & -0.474027000\end{array}$

$\begin{array}{llll}\text { C } & -1.434923000 & 1.267182000 & -0.066744000\end{array}$

$\begin{array}{llll}\text { H } & -2.511563000 & 1.144728000 & 0.113704000\end{array}$

C $\quad-2.940699000 \quad-0.692532000 \quad-1.656737000$

$\begin{array}{llll}\text { C } & -3.271501000 & 0.515231000 & -2.321294000\end{array}$

$\begin{array}{llll}\text { H } & -2.509712000 & 1.294985000 & -2.418230000\end{array}$

$\begin{array}{llll}\text { C } & -4.564863000 & 0.716839000 & -2.825958000\end{array}$

H $\quad-4.806707000 \quad 1.663250000 \quad-3.325613000$

$\begin{array}{llll}\text { C } & -5.548670000 & -0.280247000 & -2.686316000\end{array}$

H $\quad-6.562277000 \quad-0.113417000-3.072144000$

C $\quad-5.225130000 \quad-1.494560000 \quad-2.051065000$

H $\quad-5.983689000 \quad-2.279839000 \quad-1.941447000$

C $\quad-3.935670000 \quad-1.700642000 \quad-1.539931000$

H $\quad-3.685339000 \quad-2.636881000-1.025940000$

C $\quad-1.638255000 \quad-0.969757000 \quad-1.087647000$

$\begin{array}{llll}\text { C } & -0.709604000 & -1.792790000 & -0.802070000\end{array}$

$\begin{array}{llll}\text { H } & -0.190049000 & -2.730758000 & -0.990118000\end{array}$ 
${ }^{1}$ 5III-ABA $\quad$ FSPE: - 3185.72297476042 $E_{\mathrm{h}}$

\begin{tabular}{|c|c|c|c|}
\hline & 4.521835000 & 4.827558000 & 3.133756000 \\
\hline O & 2.799078000 & 6.048829000 & 5.926886000 \\
\hline 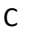 & 7.050877000 & 3.507506000 & 2.586609000 \\
\hline H & 7.718917000 & 2.643916000 & 2.738280000 \\
\hline 0 & 5.541585000 & 5.952181000 & 1.998710000 \\
\hline C & 7.492145000 & 4.526407000 & 1.672068000 \\
\hline 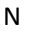 & 4.071570000 & 4.097445000 & 4.983673000 \\
\hline C & 8.750886000 & 4.374296000 & 1.024632000 \\
\hline $\mathrm{H}$ & 9.324876000 & 3.456126000 & 1.210169000 \\
\hline $\mathrm{N}$ & 5.945881000 & 3.547212000 & 3.277173000 \\
\hline C & 9.260632000 & 5.361387000 & 0.181531000 \\
\hline $\mathrm{H}$ & 10.230990000 & 5.227338000 & -0.310781000 \\
\hline C & 8.507487000 & 6.545402000 & -0.022734000 \\
\hline $\mathrm{H}$ & 8.898051000 & 7.333805000 & -0.679642000 \\
\hline C & 7.269164000 & 6.724084000 & 0.595701000 \\
\hline $\mathrm{H}$ & 6.685794000 & 7.638296000 & 0.435891000 \\
\hline C & 6.715808000 & 5.721583000 & 1.450267000 \\
\hline C & 5.617038000 & 2.409872000 & 4.148980000 \\
\hline $\mathrm{H}$ & 6.507038000 & 1.801093000 & 4.386298000 \\
\hline $\mathrm{H}$ & 4.894297000 & 1.769983000 & 3.605773000 \\
\hline C & 5.004836000 & 3.013699000 & 5.397633000 \\
\hline $\mathrm{H}$ & 4.453559000 & 2.273936000 & 6.003256000 \\
\hline $\mathrm{H}$ & 5.772781000 & 3.482330000 & 6.029926000 \\
\hline C & 2.946748000 & 3.731197000 & 4.295311000 \\
\hline C & 1.688590000 & 4.408899000 & 4.532498000 \\
\hline 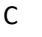 & 0.478995000 & 3.948763000 & 3.959814000 \\
\hline $\mathrm{H}$ & 0.502574000 & 3.057399000 & 3.318943000 \\
\hline C & -0.728523000 & 4.616605000 & 4.198929000 \\
\hline H & -1.659768000 & 4.251891000 & 3.749315000 \\
\hline c & -0.736111000 & 5.759395000 & 5.029534000 \\
\hline $\mathrm{H}$ & -1.677455000 & 6.288733000 & 5.224843000 \\
\hline c & 0.450578000 & 6.227470000 & 5.613346000 \\
\hline $\mathrm{H}$ & 0.455394000 & 7.110813000 & 6.263848000 \\
\hline C & 1.673277000 & 5.566180000 & 5.364677000 \\
\hline $\mathrm{H}$ & 2.909850000 & 2.696457000 & 3.937849000 \\
\hline $\mathrm{H}$ & 2.505748000 & 2.393030000 & 2.070712000 \\
\hline B & 4.167467000 & 5.504719000 & 5.722260000 \\
\hline 0 & 4.970090000 & 6.307226000 & 4.780750000 \\
\hline 0 & 4.895309000 & 5.468895000 & 6.964998000 \\
\hline C & 6.199180000 & 6.746289000 & 5.444814000 \\
\hline C & 5.787617000 & 6.623662000 & 6.964930000 \\
\hline C & 4.999395000 & 7.852725000 & 7.453174000 \\
\hline C & 6.951608000 & 6.331579000 & 7.913081000 \\
\hline C & 6.511275000 & 8.165559000 & 4.968817000 \\
\hline C & 7.364636000 & 5.825903000 & 5.069033000 \\
\hline $\mathrm{H}$ & 6.753057000 & 8.140428000 & 3.892336000 \\
\hline $\mathrm{H}$ & 5.650568000 & 8.836733000 & 5.099680000 \\
\hline $\mathrm{H}$ & 7.474606000 & 5.817287000 & 3.974408000 \\
\hline $\mathrm{H}$ & 5.655571000 & 8.734055000 & 7.563040000 \\
\hline $\mathrm{H}$ & 4.183955000 & 8.104557000 & 6.755007000 \\
\hline $\mathrm{H}$ & 7.203806000 & 4.791200000 & 5.407371000 \\
\hline $\mathrm{H}$ & 8.308630000 & 6.194956000 & 5.505674000 \\
\hline $\mathrm{H}$ & 7.377735000 & 8.576456000 & 5.518037000 \\
\hline $\mathrm{H}$ & 4.556534000 & 7.625293000 & 8.438853000 \\
\hline $\mathrm{H}$ & 7.715557000 & 7.127027000 & 7.847625000 \\
\hline $\mathrm{H}$ & 7.426866000 & 5.364646000 & 7.679778000 \\
\hline $\mathrm{H}$ & 6.585424000 & 6.293860000 & 8.954582000 \\
\hline r & 3.190209000 & 1.914735000 & 0.072296000 \\
\hline C & 4.189785000 & 1.966638000 & -0.936827000 \\
\hline$H$ & 5.080356000 & 2.590280000 & -0.785142000 \\
\hline C & 4.067797000 & 1.221546000 & -2.119107000 \\
\hline$H$ & 4.857143000 & 1.281658000 & -2.880124000 \\
\hline 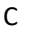 & 2.948933000 & 0.390077000 & -2.330693000 \\
\hline $\mathrm{H}$ & 2.858698000 & -0.195274000 & -3.254501000 \\
\hline
\end{tabular}

Page S73 


$\begin{array}{llll}\text { C } & 1.955029000 & 0.313457000 & -1.337171000 \\ \text { H } & 1.079873000 & -0.333563000 & -1.482748000 \\ \text { C } & 2.076560000 & 1.062892000 & -0.154874000 \\ \text { H } & 1.293100000 & 0.995727000 & 0.613034000 \\ \text { C } & 3.263933000 & 2.687214000 & 1.329851000 \\ \text { C } & 4.125554000 & 3.702190000 & 1.598062000 \\ \text { H } & 4.824412000 & 3.982122000 & 0.788516000 \\ \text { C } & 3.513667000 & 8.221292000 & 3.104701000 \\ \text { C } & 4.387931000 & 8.956826000 & 2.260494000 \\ \text { H } & 4.788475000 & 8.467130000 & 1.367448000 \\ \text { C } & 4.738860000 & 10.274197000 & 2.584378000 \\ \text { H } & 5.421579000 & 10.831533000 & 1.930921000 \\ \text { C } & 4.220004000 & 10.880750000 & 3.745293000 \\ \text { H } & 4.496881000 & 11.912302000 & 3.996462000 \\ \text { C } & 3.347819000 & 10.160535000 & 4.586071000 \\ \text { H } & 2.948320000 & 10.628471000 & 5.494302000 \\ \text { C } & 2.997939000 & 8.839762000 & 4.275400000 \\ \text { H } & 2.339981000 & 8.264512000 & 4.933245000 \\ \text { C } & 3.123215000 & 6.902115000 & 2.747635000 \\ \text { C } & 2.750791000 & 5.776420000 & 2.347801000 \\ \text { H } & 2.006342000 & 5.162310000 & 1.854753000\end{array}$

\section{${ }^{1}$ IIII-ABB}

Fe $\quad 4.713587000$

O 2.839268000

C $\quad 6.989661000$

H $\quad 7.659801000$

O 5.550121000

C $\quad 7.291774000$

N 4.164706000

C 8.379359000

H 8.937532000

N 5.980978000

C 8.742246000

H 9.577991000

C 8.024177000

H 8.303300000

C $\quad 6.958184000$

H 6.405996000

C 6.551792000

C $\quad 5.706650000$

H $\quad 6.630127000$

H $\quad 5.017510000$

C $\quad 5.067696000$

H 4.504272000

H $\quad 5.827037000$

C $\quad 3.129247000$

C 1.955828000

C 0.909546000

H $\quad 1.007209000$

C $\quad-0.223197000$

H $\quad-1.024057000$

C -0.329199000

H -1.216851000

C $\quad 0.695759000$

H $\quad 0.631825000$

C $\quad 1.853261000$

H 2.990371000

H 4.717798000

B $\quad 4.217258000$

O 4.891684000

O 5.035322000

C $\quad 6.125259000$
FSPE: - 3185.72751687981 $E_{\mathrm{h}}$

$4.418913000 \quad 3.423439000$

$5.592702000 \quad 6.195186000$

$3.018729000 \quad 2.603096000$

$2.152010000 \quad 2.710153000$

$\begin{array}{ll}5.549656000 & 2.226996000\end{array}$

$3.978229000 \quad 1.568808000$

$3.706380000 \quad 5.206553000$

$3.723426000 \quad 0.687440000$

$2.784633000 \quad 0.804657000$

$3.109036000 \quad 3.435364000$

$4.631232000-0.310931000$

$\begin{array}{ll}4.408525000 & -0.984926000\end{array}$

$5.844342000-0.435205000$

$6.569767000-1.210578000$

$\begin{array}{ll}6.132531000 & 0.423328000\end{array}$

$7.077189000 \quad 0.345017000$

$5.209585000 \quad 1.427083000$

$2.000784000 \quad 4.376429000$

$1.449950000 \quad 4.626511000$

$1.297229000 \quad 3.871423000$

$2.606109000 \quad 5.620502000$

$1.854404000 \quad 6.200611000$

$3.060987000 \quad 6.275106000$

$3.455137000 \quad 4.326708000$

$4.326871000 \quad 4.328666000$

$4.139159000 \quad 3.394374000$

$3.340665000 \quad 2.647531000$

$4.960125000 \quad 3.414619000$

$4.809941000 \quad 2.681106000$

$5.977132000 \quad 4.388808000$

$6.621935000 \quad 4.413386000$

$6.179287000 \quad 5.323596000$

$6.971134000 \quad 6.079719000$

$5.373210000 \quad 5.289153000$

$2.417626000 \quad 3.997905000$

$1.659579000 \quad 1.634610000$

$5.127590000 \quad 5.928595000$

$5.962324000 \quad 4.889022000$

\begin{tabular}{ll}
$5.178461000 \quad 7.100909000$ \\
\hline
\end{tabular}

$6.522439000 \quad 5.470083000$

Page S74 


$\begin{array}{llll}\text { C } & 5.817744000 & 6.411124000 & 7.015619000 \\ \text { C } & 4.958148000 & 7.580533000 & 7.527600000 \\ \text { C } & 7.060190000 & 6.248285000 & 7.892617000 \\ \text { C } & 6.282436000 & 7.942455000 & 4.928179000 \\ \text { C } & 7.320628000 & 5.661641000 & 5.047925000 \\ \text { H } & 6.449033000 & 7.891032000 & 3.838207000 \\ \text { H } & 5.379900000 & 8.547961000 & 5.109255000 \\ \text { H } & 7.370756000 & 5.622229000 & 3.949026000 \\ \text { H } & 5.540765000 & 8.517532000 & 7.569167000 \\ \text { H } & 4.072614000 & 7.738144000 & 6.889090000 \\ \text { H } & 7.226251000 & 4.630792000 & 5.425129000 \\ \text { H } & 8.265042000 & 6.091420000 & 5.422800000 \\ \text { H } & 7.147064000 & 8.445631000 & 5.397169000 \\ \text { H } & 4.608002000 & 7.345445000 & 8.547957000 \\ \text { H } & 7.747498000 & 7.101932000 & 7.754011000 \\ \text { H } & 7.599470000 & 5.316695000 & 7.656115000 \\ \text { H } & 6.764105000 & 6.215751000 & 8.956084000 \\ \text { C } & 3.162347000 & 1.946820000 & 0.139493000 \\ \text { C } & 2.049473000 & 2.680030000 & -0.354708000 \\ \text { H } & 1.771772000 & 3.632551000 & 0.113576000 \\ \text { C } & 1.297999000 & 2.212301000 & -1.441774000 \\ \text { H } & 0.450101000 & 2.808977000 & -1.802306000 \\ \text { C } & 1.624819000 & 0.993607000 & -2.070472000 \\ \text { H } & 1.032218000 & 0.629433000 & -2.919391000 \\ \text { C } & 2.720567000 & 0.249716000 & -1.593040000 \\ \text { H } & 2.989332000 & -0.702247000 & -2.069822000 \\ \text { C } & 3.475333000 & 0.720057000 & -0.505104000 \\ \text { H } & 4.329128000 & 0.131093000 & -0.141594000 \\ \text { C } & 3.975896000 & 2.394744000 & 1.285403000 \\ \text { C } & 3.874270000 & 3.591737000 & 1.913149000 \\ \text { H } & 3.150333000 & 4.333674000 & 1.531455000 \\ \text { C } & 2.148806000 & 6.521047000 & 0.565113000 \\ \text { C } & 0.783021000 & 6.356240000 & 0.215792000 \\ \text { H } & 0.012255000 & 6.557796000 & 0.968510000 \\ \text { C } & 0.430312000 & 5.930670000 & -1.074632000 \\ \text { H } & -0.628291000 & 5.804365000 & -1.333643000 \\ \text { C } & 1.427330000 & 5.658610000 & -2.030311000 \\ \text { H } & 1.147513000 & 5.317463000 & -3.034401000 \\ \text { C } & 2.785205000 & 5.799366000 & -1.686140000 \\ \text { H } & 3.565733000 & 5.565042000 & -2.420482000 \\ \text { C } & 3.148525000 & 6.227046000 & -0.401597000 \\ \text { H } & 4.202770000 & 6.313453000 & -0.115910000 \\ \text { C } & 2.533062000 & 6.954296000 & 1.872360000 \\ \text { C } & 2.915786000 & 7.308863000 & 2.982873000 \\ \text { H } & 3.303580000 & 7.541716000 & 3.962255000\end{array}$

\section{TS( ${ }^{1}$ IIII-AAA, ${ }^{1}$ 5IV-AAA) FSPE: -3185.70577246185 $E_{\mathrm{h}}$}

$\begin{array}{lrrr}\text { Fe } & -0.172807000 & -0.792132000 & -0.049316000 \\ \text { O } & -1.900601000 & 0.798864000 & 2.640097000 \\ \text { C } & 2.279697000 & -2.461525000 & -0.275004000 \\ \text { H } & 2.850138000 & -3.361422000 & 0.012101000 \\ \text { O } & 1.098688000 & 0.065889000 & -1.185376000 \\ \text { C } & 2.833490000 & -1.646998000 & -1.325278000 \\ \text { N } & -0.763872000 & -1.316796000 & 1.884476000 \\ \text { C } & 4.005884000 & -2.085471000 & -1.994768000 \\ \text { H } & 4.455423000 & -3.039574000 & -1.687828000 \\ \text { N } & 1.163340000 & -2.212773000 & 0.344105000 \\ \text { C } & 4.571867000 & -1.343764000 & -3.033119000 \\ \text { H } & 5.464705000 & -1.706746000 & -3.555549000 \\ \text { C } & 3.969560000 & -0.117220000 & -3.405083000 \\ \text { H } & 4.404752000 & 0.480124000 & -4.217464000 \\ \text { C } & 2.816434000 & 0.340070000 & -2.763624000 \\ \text { H } & 2.335644000 & 1.280891000 & -3.058466000\end{array}$


$\begin{array}{llll}\text { C } & 2.197947000 & -0.418870000 & -1.725164000\end{array}$

$\begin{array}{llll}\text { C } & 0.692076000 & -3.196424000 & 1.325855000\end{array}$

H $\quad 1.502886000 \quad-3.854469000 \quad 1.688445000$

$\begin{array}{llll}H & -0.065883000 & -3.837811000 & 0.833909000\end{array}$

$\begin{array}{llll}\text { C } & 0.079460000 & -2.403191000 & 2.457952000\end{array}$

H $\quad-0.540984000 \quad-3.022281000 \quad 3.128605000$

$\begin{array}{llll}\text { H } & 0.857876000 & -1.908595000 & 3.057092000\end{array}$

C $\quad-1.913248000 \quad-1.667159000 \quad 1.241467000$

$\begin{array}{llll}\text { C } & -3.095682000 & -0.853348000 & 1.331767000\end{array}$

C $\quad-4.303976000 \quad-1.226817000 \quad 0.689311000$

$\begin{array}{llll}\text { H } & -4.331884000 & -2.170195000 & 0.128403000\end{array}$

$\begin{array}{llll}\text { C } & -5.437325000 & -0.410847000 & 0.768328000\end{array}$

$\begin{array}{llll}\text { H } & -6.366656000 & -0.706106000 & 0.267164000\end{array}$

$\begin{array}{llll}\text { C } & -5.376025000 & 0.795677000 & 1.503251000\end{array}$

H $\quad-6.260299000 \quad 1.441971000 \quad 1.567795000$

C $\quad-4.193163000 \quad 1.185923000 \quad 2.146932000$

$\begin{array}{llll}\text { H } & -4.130716000 & 2.128133000 & 2.704146000\end{array}$

$\begin{array}{llll}\text { C } & -3.035897000 & 0.380537000 & 2.053882000\end{array}$

H $\quad-2.008777000 \quad-2.707099000 \quad 0.904237000$

$\begin{array}{llll}\text { B } & -0.570052000 & 0.149844000 & 2.485228000\end{array}$

$\begin{array}{llll}\text { O } & 0.286513000 & 0.790759000 & 1.471180000\end{array}$

$\begin{array}{llll}\text { O } & 0.153865000 & 0.195582000 & 3.728848000\end{array}$

$\begin{array}{llll}\text { C } & 1.527241000 & 1.245960000 & 2.097133000\end{array}$

C $\quad 1.105477000 \quad 1.297281000 \quad 3.621383000$

C $\quad 0.383434000 \quad 2.607406000 \quad 3.984398000$

$\begin{array}{llll}\text { C } \quad 2.250220000 & 1.039891000 & 4.602807000\end{array}$

C $\quad 1.876930000 \quad 2.594961000 \quad 1.463716000$

$\begin{array}{llll}\text { C } & 2.656313000 & 0.245712000 & 1.832353000\end{array}$

$\begin{array}{llll}\text { H } & 2.126175000 & 2.431570000 & 0.400780000\end{array}$

$\begin{array}{llll}\mathrm{H} & 1.023109000 & 3.289161000 & 1.506840000\end{array}$

$\begin{array}{llll}\text { H } & 2.765216000 & 0.107909000 & 0.746905000\end{array}$

H $\quad 1.083335000 \quad 3.461423000 \quad 3.999791000$

$\begin{array}{llll}H & -0.429479000 & 2.825455000 & 3.271766000\end{array}$

$\begin{array}{llll}\text { H } & 2.446516000 & -0.736720000 & 2.282469000\end{array}$

$\begin{array}{llll}H & 3.612747000 & 0.621688000 & 2.234791000\end{array}$

$\begin{array}{llll}\text { H } & 2.745743000 & 3.055414000 & 1.968290000\end{array}$

$\begin{array}{llll}H & -0.059035000 & 2.504298000 & 4.990858000\end{array}$

$\begin{array}{llll}H & 3.053995000 & 1.785774000 & 4.467546000\end{array}$

$\begin{array}{llll}\text { H } & 2.676641000 & 0.032458000 & 4.468077000\end{array}$

$\begin{array}{llll}\text { H } & 1.878442000 & 1.121346000 & 5.639771000\end{array}$

$\begin{array}{llll}\text { C } & 0.140774000 & -2.801726000 & -2.531437000\end{array}$

$\begin{array}{llll}\text { C } & 0.471531000 & -4.118872000 & -2.118477000\end{array}$

$\begin{array}{llll}\text { C } & 0.705442000 & -2.305995000 & -3.739457000\end{array}$

H $\quad 0.034607000 \quad-4.518835000 \quad-1.196379000$

H $\quad 0.474492000 \quad-1.279694000 \quad-4.046922000$

$\begin{array}{llll}\text { C } & 1.358025000 & -4.900641000 & -2.874270000\end{array}$

$\begin{array}{llll}\text { C } & 1.592503000 & -3.091167000 & -4.486847000\end{array}$

H $\quad 1.610336000 \quad-5.913413000 \quad-2.535164000$

H $\quad 2.043430000 \quad-2.679407000 \quad-5.398201000$

$\begin{array}{llll}\text { C } & 1.930970000 & -4.387903000 & -4.053393000\end{array}$

$\begin{array}{llll}\text { H } & 2.639212000 & -4.995026000 & -4.630948000\end{array}$

C $\quad-0.650703000-1.917273000 \quad-1.722983000$

C $\quad-1.594516000-1.074208000 \quad-1.487455000$

H $\quad-2.657963000 \quad-0.901976000-1.663193000$

$\begin{array}{llll}\text { C } & -1.366316000 & 0.728047000 & -0.588485000\end{array}$

C $\quad-1.065469000 \quad 1.764145000 \quad-1.423172000$

$\begin{array}{llll}\text { C } & -1.844177000 & 3.000692000 & -1.556880000\end{array}$

$\begin{array}{llll}\text { C } & -1.365318000 & 4.036585000 & -2.405739000\end{array}$

$\begin{array}{llll}\text { C } & -3.069089000 & 3.237070000 & -0.868933000\end{array}$

$\begin{array}{llll}\text { H } & -0.422707000 & 3.882004000 & -2.948887000\end{array}$

$\begin{array}{llll}H & -3.476240000 & 2.466407000 & -0.201295000\end{array}$

$\begin{array}{llll}\text { C } & -2.065828000 & 5.244179000 & -2.559377000\end{array}$

$\begin{array}{llll}\text { C } & -3.769026000 & 4.441176000 & -1.024201000\end{array}$

H $\quad-1.666617000 \quad 6.023781000 \quad-3.221479000$

H $\quad-4.711548000 \quad 4.592261000 \quad-0.481217000$

$\begin{array}{llll}\text { C } & -3.274793000 & 5.456149000 & -1.870152000\end{array}$

Page S76 
$\begin{array}{llll}H & -3.825560000 & 6.397870000 & -1.988499000\end{array}$

H $\quad-2.300434000 \quad 0.820595000 \quad-0.017386000$

$\begin{array}{llll}H & -0.150437000 & 1.697558000 & -2.027381000\end{array}$

\section{TS( ${ }^{1} 5$ III-AAB, ${ }^{1}$ IIV-AAB) FSPE: $-3185.704523375644 E_{h}$}

$\begin{array}{lrrr}\text { Fe } & 0.477138000 & -1.200360000 & -0.200311000 \\ \text { O } & -1.036373000 & 0.663407000 & 2.403707000\end{array}$

$\begin{array}{llll}\text { C } & 2.919410000 & -2.863023000 & -0.415164000\end{array}$

H $3.499642000-3.749218000 \quad-0.103707000$

$\begin{array}{llll}\text { O } & 1.611874000 & -0.473028000 & -1.542274000\end{array}$

$\begin{array}{llll}\text { C } & 3.436942000 & -2.100057000 & -1.521517000\end{array}$

N $\quad-0.046314000 \quad-1.582962000 \quad 1.835467000$

$\begin{array}{llll}\text { C } & 4.670190000 & -2.492666000 & -2.114286000\end{array}$

$\begin{array}{llll}\text { H } & 5.172636000 & -3.388636000 & -1.723784000\end{array}$

$\begin{array}{lllll}\text { N } & 1.840251000 & -2.567816000 & 0.247973000\end{array}$

$\begin{array}{llll}\text { C } & 5.243949000 & -1.766990000 & -3.158067000\end{array}$

$\begin{array}{llll}\text { H } & 6.193538000 & -2.085462000 & -3.603910000\end{array}$

$\begin{array}{llll}\text { C } & 4.579030000 & -0.606295000 & -3.630155000\end{array}$

H $\quad 5.018547000 \quad-0.020597000 \quad-4.448551000$

$\begin{array}{llll}\text { C } & 3.366950000 & -0.196822000 & -3.073661000\end{array}$

$\begin{array}{llll}\text { H } & 2.847570000 & 0.698748000 & -3.436729000\end{array}$

$\begin{array}{llll}\text { C } & 2.750651000 & -0.930654000 & -2.011398000\end{array}$

$\begin{array}{llll}\text { C } & 1.396285000 & -3.493423000 & 1.296665000\end{array}$

H $\quad 2.217156000 \quad-4.137792000 \quad 1.662125000$

$\begin{array}{lllll}\text { H } & 0.613833000 & -4.150666000 & 0.868289000\end{array}$

$\begin{array}{llll}\text { C } & 0.839016000 & -2.636236000 & 2.412700000\end{array}$

$\begin{array}{llll}H & 0.268238000 & -3.221901000 & 3.153248000\end{array}$

$\begin{array}{llll}\text { H } & 1.647713000 & -2.104797000 & 2.935797000\end{array}$

C $\quad-1.266222000-1.958389000 \quad 1.369272000$

C $\quad-2.398962000-1.087957000 \quad 1.436713000$

$\begin{array}{llll}\text { C } & -3.671728000 & -1.482642000 & 0.945490000\end{array}$

H $\quad-3.781419000 \quad-2.484112000 \quad 0.513646000$

$\begin{array}{llll}\text { C } & -4.756813000 & -0.606047000 & 0.989659000\end{array}$

$\begin{array}{llll}\text { H } & -5.730625000 & -0.913797000 & 0.591926000\end{array}$

$\begin{array}{llll}\text { C } & -4.586385000 & 0.684234000 & 1.546438000\end{array}$

$\begin{array}{llll}\text { H } & -5.434460000 & 1.379755000 & 1.580417000\end{array}$

$\begin{array}{llll}\text { C } & -3.342804000 & 1.097360000 & 2.042093000\end{array}$

$\begin{array}{llll}\text { H } & -3.196352000 & 2.104765000 & 2.448312000\end{array}$

$\begin{array}{llll}\text { C } & -2.229597000 & 0.230009000 & 1.974367000\end{array}$

$\begin{array}{llll}\text { H } & -1.409677000 & -3.016381000 & 1.115305000\end{array}$

$\begin{array}{llll}\text { B } & 0.251848000 & -0.076046000 & 2.272436000\end{array}$

$\begin{array}{llll}\text { O } & 1.103615000 & 0.407027000 & 1.174773000\end{array}$

$\begin{array}{llll}\text { O } & 1.025270000 & 0.063997000 & 3.480777000\end{array}$

$\begin{array}{llll}\text { C } & 2.361933000 & 0.921106000 & 1.705710000\end{array}$

C $\quad 1.995903000 \quad 1.125734000 \quad 3.233538000$

C $\quad 1.317827000 \quad 2.483268000 \quad 3.494283000$

C $3.170226000 \quad 0.934194000 \quad 4.195004000$

$\begin{array}{llll}\text { C } & 2.683655000 & 2.202690000 & 0.930301000\end{array}$

C $3.483029000 \quad-0.099767000 \quad 1.495960000$

$\begin{array}{llll}\text { H } & 2.912486000 & 1.936887000 & -0.116775000\end{array}$

$\begin{array}{llll}H & 1.821533000 & 2.888005000 & 0.919986000\end{array}$

$\begin{array}{llll}\text { H } & 3.560249000 & -0.332182000 & 0.423411000\end{array}$

$\begin{array}{llll}H & 2.037336000 & 3.315864000 & 3.402363000\end{array}$

$\begin{array}{llll}\text { H } & 0.483337000 & 2.653637000 & 2.794040000\end{array}$

$\begin{array}{llll}H & 3.289385000 & -1.038567000 & 2.037598000\end{array}$

$\begin{array}{llll}H & 4.451961000 & 0.309191000 & 1.830881000\end{array}$

$\begin{array}{llll}\text { H } & 3.558354000 & 2.720510000 & 1.364056000\end{array}$

$\begin{array}{llll}H & 0.912182000 & 2.488185000 & 4.521354000\end{array}$

$\begin{array}{llll}H & 3.983816000 & 1.644627000 & 3.962524000\end{array}$

$\begin{array}{llll}\text { H } & 3.570501000 & -0.091419000 & 4.141447000\end{array}$

$\begin{array}{llll}\text { H } & 2.838055000 & 1.121366000 & 5.231692000\end{array}$

$\begin{array}{llll}\text { C } & -2.357426000 & -1.506725000 & -2.219196000\end{array}$

C $\quad-3.205142000-2.647782000 \quad-2.168378000$

C $\quad-2.866273000-0.316366000 \quad-2.794276000$ 


$\begin{array}{lrrr}\text { H } & -2.822160000 & -3.570834000 & -1.715788000 \\ \text { H } & -2.222246000 & 0.565492000 & -2.855910000 \\ \text { C } & -4.517722000 & -2.590406000 & -2.657030000 \\ \text { C } & -4.183567000 & -0.261670000 & -3.275153000 \\ \text { H } & -5.157904000 & -3.479917000 & -2.596845000 \\ \text { H } & -4.560220000 & 0.673753000 & -3.708028000 \\ \text { C } & -5.018415000 & -1.392142000 & -3.202131000 \\ \text { H } & -6.051753000 & -1.341391000 & -3.568301000 \\ \text { C } & -0.108128000 & -2.556659000 & -1.443331000 \\ \text { C } & -1.008604000 & -1.664937000 & -1.686255000 \\ \text { H } & 0.294505000 & -3.530638000 & -1.726411000 \\ \text { C } & -0.778405000 & 0.262698000 & -0.706757000 \\ \text { C } & -0.375772000 & 1.440520000 & -1.267564000 \\ \text { C } & -1.179727000 & 2.666444000 & -1.325203000 \\ \text { C } & -0.564416000 & 3.884120000 & -1.728591000 \\ \text { C } & -2.562860000 & 2.714455000 & -0.988743000 \\ \text { H } & 0.501495000 & 3.874490000 & -1.995527000 \\ \text { H } & -3.077191000 & 1.797194000 & -0.678743000 \\ \text { C } & -1.285190000 & 5.088017000 & -1.782607000 \\ \text { C } & -3.281716000 & 3.916488000 & -1.042103000 \\ \text { H } & -0.779503000 & 6.011279000 & -2.094998000 \\ \text { H } & -4.346549000 & 3.920692000 & -0.774175000 \\ \text { C } & -2.650590000 & 5.114137000 & -1.437805000 \\ \text { H } & -3.216612000 & 6.053448000 & -1.478579000 \\ \text { H } & -1.806310000 & 0.237963000 & -0.321016000 \\ \text { H } & 0.653071000 & 1.520415000 & -1.642010000\end{array}$

\section{TS( ${ }^{1}$ 5III-ABA, ${ }^{1}$ 5IV-ABA) FSPE: $-3185.708855694618 E_{\mathrm{h}}$}

$\begin{array}{lrrr}\text { Fe } & -0.186020000 & -0.386314000 & -0.052606000 \\ \text { O } & -1.751718000 & 0.722156000 & 2.872495000 \\ \text { C } & 2.185410000 & -1.927675000 & -0.849906000 \\ \text { H } & 2.756184000 & -2.871246000 & -0.814635000 \\ \text { O } & 0.984582000 & 0.729606000 & -1.105372000 \\ \text { C } & 2.669893000 & -0.907642000 & -1.746425000 \\ \text { N } & -0.618792000 & -1.226995000 & 1.757824000 \\ \text { C } & 3.816137000 & -1.171574000 & -2.545969000 \\ \text { H } & 4.269150000 & -2.171380000 & -2.495189000 \\ \text { N } & 1.15732000 & -1.807545000 & -0.062017000 \\ \text { C } & 4.368190000 & -0.192489000 & -3.373939000 \\ \text { H } & 5.248231000 & -0.414176000 & -3.989060000 \\ \text { C } & 3.778122000 & 1.096311000 & -3.399725000 \\ \text { H } & 4.206565000 & 1.878948000 & -4.039997000 \\ \text { C } & 2.651365000 & 1.386429000 & -2.627506000 \\ \text { H } & 2.184746000 & 2.379007000 & -2.642397000 \\ \text { C } & 2.051944000 & 0.393105000 & -1.794343000 \\ \text { C } & 0.772274000 & -2.959331000 & 0.763065000 \\ \text { H } & 1.612145000 & -3.657825000 & 0.926858000 \\ \text { H } & -0.025067000 & -3.507158000 & 0.221314000 \\ \text { C } & 0.264481000 & -2.383269000 & 2.070490000 \\ \text { H } & -0.301436000 & -3.117187000 & 2.669899000 \\ \text { H } & 1.091913000 & -1.992599000 & 2.680983000 \\ \text { C } & -1.783476000 & -1.480548000 & 1.087481000 \\ \text { C } & -2.982521000 & -0.723639000 & 1.370417000 \\ \text { C } & -4.221867000 & -1.050577000 & 0.769115000 \\ \text { H } & -4.266960000 & -1.907791000 & 0.083961000 \\ \text { C } & -5.370756000 & -0.297065000 & 1.039593000 \\ \text { H } & -6.325875000 & -0.557851000 & 0.568496000 \\ \text { C } & -5.287654000 & 0.800001000 & 1.925565000 \\ \text { H } & -6.181755000 & 1.398312000 & 2.142021000 \\ \text { C } & -4.070433000 & 1.135623000 & 2.537302000 \\ \text { H } & -3.994249000 & 1.982936000 & 3.229529000 \\ \text { C } & -2.908382000 & 0.383314000 & 2.262453000 \\ \text { H } & -1.896348000 & -2.481537000 & 0.655065000 \\ \text { H } & -2.124301000 & -2.813899000 & -1.346977000 \\ \text { B } & -0.423214000 & 0.139288000 & 2.557966000 \\ \text { O } & 0.347283000 & 0.946240000 & 1.589173000\end{array}$




\begin{tabular}{|c|c|c|c|}
\hline & & 000 & \\
\hline & 8231000 & .312254000 & 182918000 \\
\hline & 306810000 & 1.169124000 & \\
\hline & & & \\
\hline & 2.509436000 & 0.8217 & 1000 \\
\hline & 9735 & 000 & 000 \\
\hline & 2.737215000 & 0.345 & 7000 \\
\hline & .118603000 & 2.6970 & 9000 \\
\hline & 1.1519 & 3.426 & 5000 \\
\hline & 2.797016000 & 0.350668000 & 19000 \\
\hline & 1.2781 & 3.266082000 & 7000 \\
\hline & -0.2574 & 2.7064 & 3.6 \\
\hline & 2.539 & -0.685 & 4000 \\
\hline & 3.715 & 0.66 & \\
\hline & 2.899 & 3.09 & 5000 \\
\hline & 0.189 & 2.17 & 5.2 \\
\hline & 3.294 & 1.593549000 & 3000 \\
\hline & 2.939 & -0.1553 & 1000 \\
\hline & 2.197 & 0.7759 & 3000 \\
\hline & -1.151 & -3.2218 & 3000 \\
\hline & & & \\
\hline & 0.700 & -2.251 & 2000 \\
\hline & 00 & -3.810 & -5 \\
\hline & 0.987 & -3.633 & -5. \\
\hline & -0.789 & -4.8406 & -5 \\
\hline & -0.648 & -5.462 & -6 \\
\hline & -1.87 & -5.071 & -4 \\
\hline & -2.59 & & \\
\hline & -2.053 & -4.27 & -3. \\
\hline & -2.90 & & -2 \\
\hline & -1.365 & -2.419 & -2 \\
\hline & -0.732 & -1.235 & -1 \\
\hline & -0.073 & -0.828 & -2 \\
\hline & -1.159 & 2.6677 & -0.3 \\
\hline & -0.631 & 3.4478 & -1 \\
\hline & -0.42 & 2.95 & -2 \\
\hline & -0.35 & & -1 \\
\hline & 0.055 & 5.394 & -2 \\
\hline & -0.612 & 5.431 & -0. \\
\hline & -0.398 & 6.498 & 800 \\
\hline & -1.1383 & 4.6690 & 900 \\
\hline & -1.333 & 5.1410 & 600 \\
\hline & -1.400203000 & 3.301764000 & 5400 \\
\hline & -1.776726000 & 2.705610000 & 97800 \\
\hline & -1.401644000 & 1.280283000 & -0.61236100 \\
\hline & -1.85 & 0.201122000 & -1.1 \\
\hline & -2.83879800 & -0.184594000 & -1.416039 \\
\hline
\end{tabular}

\section{TS( $\left.{ }^{1} 5 I I I-A B B,{ }^{1} 5 I V-A B B\right) \quad F S P E:-3185.704122197608 E_{h}$}

$\begin{array}{lrrr}\text { Fe } & 0.281845000 & 0.096251000 & 0.343096000 \\ \text { O } & -1.394783000 & 1.385532000 & 3.092976000 \\ \text { C } & 2.782550000 & -1.366142000 & -0.237583000 \\ \text { H } & 3.408799000 & -2.267290000 & -0.116802000 \\ \text { O } & 1.393876000 & 1.181210000 & -0.789736000 \\ \text { C } & 3.238320000 & -0.376430000 & -1.182902000 \\ \text { N } & -0.176018000 & -0.626826000 & 2.200103000 \\ \text { C } & 4.450623000 & -0.612031000 & -1.889920000 \\ \text { H } & 4.977420000 & -1.560661000 & -1.716444000 \\ \text { N } & 1.712195000 & -1.254295000 & 0.490849000 \\ \text { C } & 4.976047000 & 0.327903000 & -2.777178000 \\ \text { H } & 5.910109000 & 0.126661000 & -3.314788000 \\ \text { C } & 4.283685000 & 1.549982000 & -2.967334000 \\ \text { H } & 4.684644000 & 2.302705000 & -3.659107000 \\ \text { C } & 3.089909000 & 1.810535000 & -2.292235000 \\ \text { H } & 2.548965000 & 2.753441000 & -2.440749000 \\ \text { C } & 2.522921000 & 0.859654000 & -1.387885000 \\ \text { C } & 1.339004000 & -2.359717000 & 1.379125000 \\ \text { H } & 2.197489000 & -3.008264000 & 1.631287000\end{array}$


$\begin{array}{llll}H & 0.588355000 & -2.979311000 & 0.848138000\end{array}$

$\begin{array}{llll}\text { C } & 0.748204000 & -1.717988000 & 2.620628000\end{array}$

H $\quad 0.193313000 \quad-2.435945000 \quad 3.248705000$

$\begin{array}{llll}\text { H } & 1.530202000 & -1.248119000 & 3.234426000\end{array}$

$\begin{array}{llll}\text { C } & -1.312284000 & -0.984518000 & 1.534560000\end{array}$

$\begin{array}{llll}\text { C } & -2.537537000 & -0.237327000 & 1.700666000\end{array}$

$\begin{array}{llll}\text { C } & -3.745392000 & -0.650406000 & 1.090171000\end{array}$

H $\quad-3.743923000 \quad-1.557569000 \quad 0.472526000$

$\begin{array}{llll}\text { C } & -4.922778000 & 0.087960000 & 1.256515000\end{array}$

H $\quad-5.851024000 \quad-0.237277000 \quad 0.772438000$

$\begin{array}{llll}\text { C } & -4.899939000 & 1.261536000 & 2.042293000\end{array}$

$\begin{array}{llll}\text { H } & -5.816717000 & 1.850257000 & 2.174059000\end{array}$

$\begin{array}{llll}\text { C } & -3.714044000 & 1.688062000 & 2.659229000\end{array}$

$\begin{array}{llll}\text { H } & -3.685088000 & 2.598047000 & 3.270869000\end{array}$

$\begin{array}{llll}\text { C } & -2.522169000 & 0.950194000 & 2.492069000\end{array}$

H $\quad-1.376589000 \quad-2.024367000 \quad 1.196382000$

H $\quad-1.821244000 \quad-2.269139000 \quad-0.731712000$

$\begin{array}{llll}\text { B } & -0.044786000 & 0.797341000 & 2.901458000\end{array}$

$\begin{array}{llll}\text { O } & 0.777184000 & 1.557480000 & 1.932855000\end{array}$

$\begin{array}{llll}\text { O } & 0.691896000 & 0.771213000 & 4.137061000\end{array}$

$\begin{array}{llll}\text { C } & 2.019135000 & 1.987686000 & 2.580379000\end{array}$

C $\quad 1.619756000 \quad 1.895567000 \quad 4.109605000$

C $\quad 0.877448000 \quad 3.152221000 \quad 4.599084000$

$\begin{array}{llll}\text { C } & 2.787164000 & 1.577947000 & 5.046003000\end{array}$

$\begin{array}{llll}\text { C } & 2.329750000 & 3.398319000 & 2.073526000\end{array}$

$\begin{array}{llll}\text { C } & 3.170577000 & 1.049670000 & 2.207996000\end{array}$

$\begin{array}{llll}\text { H } & 2.587223000 & 3.346976000 & 1.000929000\end{array}$

$\begin{array}{llll}H & 1.460948000 & 4.066129000 & 2.186295000\end{array}$

$\begin{array}{llll}\text { H } & 3.264057000 & 1.013971000 & 1.112584000\end{array}$

$\begin{array}{llll}H & 1.557243000 & 4.018771000 & 4.677845000\end{array}$

$\begin{array}{llll}\text { H } & 0.044973000 & 3.412773000 & 3.924018000\end{array}$

$\begin{array}{llll}H & 3.002447000 & 0.024332000 & 2.570460000\end{array}$

$\begin{array}{llll}H & 4.123247000 & 1.418180000 & 2.625717000\end{array}$

$\begin{array}{llll}H & 3.187346000 & 3.831069000 & 2.619893000\end{array}$

$\begin{array}{llll}H & 0.457619000 & 2.949906000 & 5.600105000\end{array}$

$\begin{array}{llll}H & 3.572783000 & 2.350533000 & 4.965518000\end{array}$

$\begin{array}{llll}H & 3.231884000 & 0.596048000 & 4.815622000\end{array}$

$\begin{array}{llll}\text { H } & 2.431816000 & 1.558603000 & 6.091626000\end{array}$

$\begin{array}{llll}\text { C } & -0.956780000 & -2.960141000 & -2.587521000\end{array}$

C $\quad 0.070907000 \quad-2.912468000 \quad-3.571542000$

H $\quad 0.893310000 \quad-2.193804000-3.464392000$

C $\quad 0.058522000 \quad-3.779625000 \quad-4.671651000$

H $\quad 0.862646000 \quad-3.721577000 \quad-5.416740000$

C $\quad-0.973929000 \quad-4.728703000 \quad-4.823305000$

H $\quad-0.978733000 \quad-5.407852000 \quad-5.685208000$

C $\quad-1.993004000 \quad-4.800843000-3.854193000$

H $\quad-2.800429000 \quad-5.537122000-3.959162000$

C $\quad-1.982620000 \quad-3.931496000-2.752837000$

$\begin{array}{llll}\text { H } & -2.784474000 & -3.990690000 & -2.004091000\end{array}$

C $\quad-1.009065000-2.056475000 \quad-1.439591000$

$\begin{array}{llll}\text { C } & -0.224100000 & -0.943594000 & -1.247197000\end{array}$

H $\quad 0.517782000 \quad-0.728701000 \quad-2.035437000$

$\begin{array}{llll}\text { C } & -2.289450000 & 0.655638000 & -2.216960000\end{array}$

$\begin{array}{llll}\text { C } & -1.942876000 & 0.205536000 & -3.514949000\end{array}$

H $\quad-0.929342000 \quad-0.157240000 \quad-3.713062000$

$\begin{array}{llll}\text { C } & -2.890365000 & 0.217215000 & -4.548951000\end{array}$

H $\quad-2.602713000 \quad-0.135386000 \quad-5.547505000$

C $\quad-4.201870000 \quad 0.673402000 \quad-4.311424000$

$\begin{array}{llll}\text { H } & -4.942664000 & 0.670838000 & -5.120977000\end{array}$

$\begin{array}{llll}\text { C } & -4.556810000 & 1.124802000 & -3.026317000\end{array}$

H $\quad-5.578211000 \quad 1.473316000 \quad-2.826180000$

$\begin{array}{llll}\text { C } & -3.613349000 & 1.115571000 & -1.986466000\end{array}$

H $\quad-3.892740000 \quad 1.437697000 \quad-0.976435000$

C $\quad-1.081681000 \quad 1.448273000 \quad-0.055507000$

$\begin{array}{llll}\text { C } & -1.355779000 & 0.735379000 & -1.096209000\end{array}$

H $\quad-1.310836000 \quad 2.397008000 \quad 0.428497000$ 
${ }^{1}$ 5IV-AAA $\quad$ FSPE: $-\mathbf{3 1 8 5 . 7 9 1 5 6 0 5 8 5 1 7 6} E_{\mathrm{h}}$

\begin{tabular}{|c|c|c|c|}
\hline & 5.160758000 & 4.364243000 & 3.302375000 \\
\hline $\mathrm{O}$ & 3.215359000 & 6.092184000 & 5.694890000 \\
\hline C & 7.295063000 & 2.555186000 & 2.970126000 \\
\hline 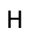 & 7.791646000 & 1.615860000 & 3.257315000 \\
\hline 0 & 6.389213000 & 5.219760000 & 2.224179000 \\
\hline C & 7.911285000 & 3.349323000 & 1.938589000 \\
\hline $\mathrm{N}$ & 4.310481000 & 3.921498000 & 5.055919000 \\
\hline C & 9.040737000 & 2.836225000 & 1.245128000 \\
\hline $\mathrm{H}$ & 9.414386000 & 1.840301000 & 1.517945000 \\
\hline $\mathrm{N}$ & 6.185997000 & 2.866373000 & 3.592782000 \\
\hline C & 9.659421000 & 3.562417000 & 0.224917000 \\
\hline $\mathrm{H}$ & 10.522235000 & 3.144654000 & -0.307364000 \\
\hline C & 9.159693000 & 4.842660000 & -0.114566000 \\
\hline $\mathrm{H}$ & 9.636096000 & 5.420855000 & -0.917010000 \\
\hline C & 8.059657000 & 5.382213000 & 0.560660000 \\
\hline $\mathrm{H}$ & 7.665120000 & 6.373572000 & 0.306922000 \\
\hline C & 7.407601000 & 4.652824000 & 1.591301000 \\
\hline c & 5.631581000 & 1.920155000 & 4.583189000 \\
\hline $\mathrm{H}$ & 6.420569000 & 1.267290000 & 4.995160000 \\
\hline $\mathrm{H}$ & 4.902333000 & 1.277110000 & 4.056128000 \\
\hline C & 4.973175000 & 2.747848000 & 5.677269000 \\
\hline $\mathrm{H}$ & 4.231072000 & 2.163443000 & 6.248877000 \\
\hline $\mathrm{H}$ & 5.720715000 & 3.148075000 & 6.378870000 \\
\hline C & 3.320680000 & 3.716497000 & 4.117083000 \\
\hline 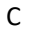 & 2.236278000 & 4.677895000 & 3.985903000 \\
\hline 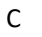 & 1.177461000 & 4.456500000 & 3.075057000 \\
\hline $\mathrm{H}$ & 1.195827000 & 3.548732000 & 2.459506000 \\
\hline C & 0.151095000 & 5.396821000 & 2.933379000 \\
\hline $\mathrm{H}$ & -0.655733000 & 5.228546000 & 2.209948000 \\
\hline c & 0.161809000 & 6.563602000 & 3.730169000 \\
\hline $\mathrm{H}$ & -0.630845000 & 7.313178000 & 3.614874000 \\
\hline C & 1.182756000 & 6.783467000 & 4.663751000 \\
\hline $\mathrm{H}$ & 1.214552000 & 7.695206000 & 5.270762000 \\
\hline C & 2.235763000 & 5.854498000 & 4.789009000 \\
\hline $\mathrm{H}$ & 3.097335000 & 2.674082000 & 3.863599000 \\
\hline B & 4.506532000 & 5.378749000 & 5.681755000 \\
\hline 0 & 5.477041000 & 5.993720000 & 4.729303000 \\
\hline 0 & 5.133007000 & 5.388004000 & 6.971619000 \\
\hline C & 6.697266000 & 6.355215000 & 5.468389000 \\
\hline C & 6.147837000 & 6.438440000 & 6.946005000 \\
\hline C & 5.467812000 & 7.787376000 & 7.242344000 \\
\hline C & 7.189427000 & 6.124541000 & 8.020977000 \\
\hline C & 7.226798000 & 7.667380000 & 4.889924000 \\
\hline$C$ & 7.743940000 & 5.249860000 & 5.288417000 \\
\hline $\mathrm{H}$ & 7.543462000 & 7.499095000 & 3.845964000 \\
\hline $\mathrm{H}$ & 6.459602000 & 8.457308000 & 4.892516000 \\
\hline $\mathrm{H}$ & 7.936147000 & 5.105534000 & 4.214191000 \\
\hline $\mathrm{H}$ & 6.207433000 & 8.603395000 & 7.319980000 \\
\hline $\mathrm{H}$ & 4.731098000 & 8.044775000 & 6.462466000 \\
\hline $\mathrm{H}$ & 7.395696000 & 4.292395000 & 5.706604000 \\
\hline $\mathrm{H}$ & 8.693697000 & 5.523635000 & 5.778652000 \\
\hline $\mathrm{H}$ & 8.098084000 & 8.021688000 & 5.469670000 \\
\hline $\mathrm{H}$ & 4.933129000 & 7.712862000 & 8.205423000 \\
\hline $\mathrm{H}$ & 8.043453000 & 6.821730000 & 7.949993000 \\
\hline $\mathrm{H}$ & 7.565535000 & 5.092897000 & 7.926070000 \\
\hline $\mathrm{H}$ & 6.737843000 & 6.236676000 & 9.022557000 \\
\hline C & 4.612798000 & 2.278440000 & 1.212274000 \\
\hline 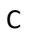 & 3.926891000 & 1.149729000 & 1.725857000 \\
\hline 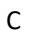 & 5.598522000 & 2.055711000 & 0.217467000 \\
\hline$H$ & 3.118127000 & 1.289817000 & 2.453463000 \\
\hline$H$ & 6.129350000 & 2.918679000 & -0.201511000 \\
\hline C & 4.239622000 & -0.150254000 & 1.292585000 \\
\hline C & 5.915800000 & 0.757192000 & -0.213729000 \\
\hline $\mathrm{H}$ & 3.688970000 & -1.005800000 & 1.704562000 \\
\hline
\end{tabular}

Page $\$ 81$ 


$\begin{array}{llll}\text { H } & 6.692671000 & 0.614848000 & -0.976102000 \\ \mathrm{C} & 5.245253000 & -0.354381000 & 0.329182000 \\ \mathrm{H} & 5.494881000 & -1.369982000 & -0.003406000 \\ \mathrm{C} & 4.336540000 & 3.660177000 & 1.671349000 \\ \mathrm{C} & 3.618103000 & 4.499068000 & 0.852647000 \\ \mathrm{H} & 3.230488000 & 4.042166000 & -0.074692000 \\ \mathrm{C} & 3.211932000 & 5.879442000 & 1.055652000 \\ \mathrm{C} & 3.650157000 & 6.741215000 & 2.021116000 \\ \mathrm{C} & 3.122849000 & 8.062072000 & 2.357554000 \\ \mathrm{C} & 3.637584000 & 8.713209000 & 3.510040000 \\ \mathrm{C} & 2.085453000 & 8.711161000 & 1.637584000 \\ \mathrm{H} & 4.411134000 & 8.197153000 & 4.092046000 \\ \mathrm{H} & 1.654773000 & 8.233851000 & 0.748543000 \\ \mathrm{C} & 3.132289000 & 9.949928000 & 3.936432000 \\ \mathrm{C} & 1.583237000 & 9.949251000 & 2.061367000 \\ \mathrm{H} & 3.539688000 & 10.422107000 & 4.840216000 \\ \mathrm{H} & 0.776684000 & 10.429809000 & 1.492032000 \\ \mathrm{C} & 2.098955000 & 10.577131000 & 3.214762000 \\ \mathrm{H} & 1.697298000 & 11.543717000 & 3.544710000 \\ \mathrm{H} & 2.416946000 & 6.225633000 & 0.378137000 \\ \mathrm{H} & 4.482537000 & 6.438218000 & 2.671974000\end{array}$

\section{${ }^{1}$ 5IV-AAB $\quad$ FSPE: $-3185.787808673922 E_{\mathrm{h}}$}

$\begin{array}{lrrr}\text { Fe } & -0.026477000 & 0.090508000 & -0.024714000 \\ \text { O } & -2.073808000 & 1.967055000 & 2.161774000 \\ \text { C } & 1.966849000 & -1.871874000 & -0.291017000 \\ \text { H } & 2.398718000 & -2.833493000 & 0.027033000 \\ \text { O } & 1.270566000 & 0.837879000 & -1.111484000 \\ \text { C } & 2.618668000 & -1.167190000 & -1.367775000 \\ \text { N } & -0.978254000 & -0.246882000 & 1.694894000 \\ \text { C } & 3.703202000 & -1.785853000 & -2.049664000 \\ \text { H } & 3.993977000 & -2.803679000 & -1.756663000 \\ \text { N } & 0.910769000 & -1.440884000 & 0.353500000 \\ \text { C } & 4.390623000 & -1.128064000 & -3.072610000 \\ \text { H } & 5.218114000 & -1.625445000 & -3.592333000 \\ \text { C } & 4.010836000 & 0.189008000 & -3.426173000 \\ \text { H } & 4.544318000 & 0.715718000 & -4.228117000 \\ \text { C } & 2.958730000 & 0.830653000 & -2.764448000 \\ \text { H } & 2.658356000 & 1.852190000 & -3.027544000 \\ \text { C } & 2.234288000 & 0.171716000 & -1.734449000 \\ \text { C } & 0.284031000 & -2.309654000 & 1.373680000 \\ \text { H } & 1.026929000 & -2.979276000 & 1.840510000 \\ \text { H } & -0.466870000 & -2.941591000 & 0.861975000 \\ \text { C } & -0.360949000 & -1.394216000 & 2.404177000 \\ \text { H } & -1.127128000 & -1.918479000 & 3.001851000 \\ \text { H } & 0.390826000 & -0.971140000 & 3.087743000 \\ \text { C } & -1.925372000 & -0.488811000 & 0.719353000 \\ \text { C } & -2.997562000 & 0.469136000 & 0.495820000 \\ \text { C } & -4.027266000 & 0.201039000 & -0.436760000 \\ \text { H } & -3.990259000 & -0.736578000 & -1.005519000 \\ \text { C } & -5.050009000 & 1.129881000 & -0.654809000 \\ \text { H } & -5.833098000 & 0.924759000 & -1.394555000 \\ \text { C } & -5.066676000 & 2.333728000 & 0.085757000 \\ \text { H } & -5.856584000 & 3.074157000 & -0.090222000 \\ \text { C } & -4.074466000 & 2.602959000 & 1.036217000 \\ \text { H } & -4.063753000 & 3.542561000 & 1.599719000 \\ \text { C } & -3.022934000 & 1.684914000 & 1.237013000 \\ \text { H } & -2.147346000 & -1.539978000 & 0.495176000 \\ \text { B } & -0.789552000 & 1.247736000 & 2.229254000 \\ \text { O } & 0.210372000 & 1.790265000 & 1.256595000 \\ \text { O } & -0.192058000 & 1.343883000 & 3.527965000 \\ \text { C } & 1.415217000 & 2.197517000 & 2.003754000 \\ \text { C } & 0.820139000 & 2.395595000 & 3.451331000\end{array}$


$\begin{array}{llll}\text { C } & 0.129330000 & 3.761680000 & 3.614798000\end{array}$

$\begin{array}{llll}\text { C } & 1.827021000 & 2.173292000 & 4.580555000\end{array}$

$\begin{array}{llll}\text { C } & 1.972378000 & 3.457385000 & 1.342408000\end{array}$

$\begin{array}{llll}\text { C } & 2.455587000 & 1.072765000 & 1.944861000\end{array}$

$\begin{array}{llll}\text { H } & 2.315603000 & 3.207120000 & 0.323637000\end{array}$

H $\quad 1.212152000 \quad 4.250399000 \quad 1.264243000$

H $\quad 2.685634000 \quad 0.844660000 \quad 0.892841000$

$\begin{array}{llll}H & 0.867338000 & 4.581667000 & 3.659314000\end{array}$

$\begin{array}{llll}H & -0.573938000 & 3.957132000 & 2.787340000\end{array}$

$\begin{array}{llll}\text { H } & 2.081320000 & 0.153227000 & 2.422267000\end{array}$

$\begin{array}{llll}H & 3.389305000 & 1.376153000 & 2.448190000\end{array}$

$\begin{array}{llll}H & 2.830340000 & 3.848974000 & 1.917967000\end{array}$

$\begin{array}{llll}\text { H } & -0.443815000 & 3.761026000 & 4.558395000\end{array}$

$\begin{array}{llll}H & 2.682985000 & 2.864313000 & 4.479444000\end{array}$

$\begin{array}{llll}H & 2.205299000 & 1.138112000 & 4.583173000\end{array}$

$\begin{array}{llll}\text { H } & 1.343773000 & 2.366616000 & 5.554565000\end{array}$

C $\quad-1.989879000 \quad-1.007100000 \quad-3.695519000$

C $\quad-2.443967000 \quad-2.339133000 \quad-3.521415000$

C $\quad-2.022587000 \quad-0.463716000 \quad-5.004820000$

H $\quad-2.466467000 \quad-2.768377000 \quad-2.511247000$

$\begin{array}{llll}H & -1.672302000 & 0.564389000 & -5.167498000\end{array}$

C $\quad-2.890673000 \quad-3.103004000 \quad-4.612311000$

C $\quad-2.474032000 \quad-1.224830000 \quad-6.097056000$

H $\quad-3.242577000 \quad-4.129994000-4.448202000$

H $\quad-2.481747000 \quad-0.781948000-7.101700000$

C $\quad-2.907614000 \quad-2.550468000 \quad-5.907689000$

H $\quad-3.262550000 \quad-3.144697000 \quad-6.759335000$

$\begin{array}{llll}\text { C } & -0.767736000 & -0.790287000 & -1.547913000\end{array}$

C $\quad-1.506293000 \quad-0.185791000 \quad-2.541854000$

H $\quad-0.543010000 \quad-1.865767000-1.665838000$

$\begin{array}{llll}\text { C } & -1.938490000 & 1.215325000 & -2.515265000\end{array}$

$\begin{array}{llll}\text { C } & -1.462581000 & 2.208614000 & -1.707447000\end{array}$

C $\quad-2.068094000 \quad 3.517051000 \quad-1.457567000$

C $\quad-1.567514000 \quad 4.287522000 \quad-0.375133000$

$\begin{array}{llll}\text { C } & -3.170519000 & 4.033605000 & -2.187205000\end{array}$

$\begin{array}{llll}\text { H } & -0.743481000 & 3.872922000 & 0.219141000\end{array}$

$\begin{array}{llll}H & -3.586141000 & 3.462039000 & -3.026372000\end{array}$

C $\quad-2.156016000 \quad 5.509550000 \quad-0.018174000$

$\begin{array}{llll}\text { C } & -3.754658000 & 5.257700000 & -1.834339000\end{array}$

$\begin{array}{llll}\text { H } & -1.761935000 & 6.074544000 & 0.836825000\end{array}$

$\begin{array}{llll}\text { H } & -4.611659000 & 5.634663000 & -2.407807000\end{array}$

$\begin{array}{llll}\text { C } & -3.256580000 & 6.002084000 & -0.744524000\end{array}$

$\begin{array}{llll}\text { H } & -3.723110000 & 6.956008000 & -0.467209000\end{array}$

H $\quad-2.818871000 \quad 1.437595000 \quad-3.136827000$

H $\quad-0.549059000 \quad 2.031930000-1.124901000$

\section{${ }^{1}$ 5IV-ABA $\quad$ FSPE: $-3185.771915133326 E_{\mathrm{h}}$}

$\begin{array}{llll}\text { Fe } & 0.000000000 & 0.000000000 & 0.000000000\end{array}$

$\begin{array}{llll}\text { O } & -1.548494000 & 0.419006000 & 3.037108000\end{array}$

C $\quad 2.129367000 \quad-1.621597000 \quad-1.278498000$

H $\quad 2.541585000 \quad-2.607405000 \quad-1.554493000$

$\begin{array}{llll}\text { O } & 1.238604000 & 1.175273000 & -0.869647000\end{array}$

$\begin{array}{llll}\text { C } & 2.800382000 & -0.461874000 & -1.812238000\end{array}$

$\begin{array}{llll}\mathrm{N} & -0.666585000 & -1.270158000 & 1.401140000\end{array}$

$\begin{array}{llll}\text { C } \quad 3.980128000 & -0.647824000 & -2.588982000\end{array}$

H $\quad 4.304276000 \quad-1.675686000 \quad-2.803275000$

$\begin{array}{llll}\text { N } & 1.106509000 & -1.574518000 & -0.471855000\end{array}$

$\begin{array}{llll}\text { C } & 4.724554000 & 0.433948000 & -3.056786000\end{array}$

$\begin{array}{llll}\text { H } & 5.629614000 & 0.270322000 & -3.653576000\end{array}$

$\begin{array}{llll}\text { C } & 4.301037000 & 1.748858000 & -2.738617000\end{array}$

H $\quad 4.883096000 \quad 2.610665000 \quad-3.090954000$

$\begin{array}{llll}\text { C } \quad 3.146775000 & 1.965663000 & -1.984712000\end{array}$

H $2.809101000 \quad 2.9801390000-1.739005000$ 
$\begin{array}{llll}\text { C } & 2.346639000 & 0.876235000 & -1.523361000\end{array}$

$\begin{array}{llll}\text { C } & 0.524436000 & -2.850862000 & -0.024576000\end{array}$

H $\quad 1.262972000 \quad-3.672854000 \quad-0.032755000$

H $\quad-0.287480000 \quad-3.123806000-0.727332000$

$\begin{array}{llll}\text { C } & -0.012513000 & -2.603619000 & 1.373503000\end{array}$

H $\quad-0.743530000 \quad-3.369119000 \quad 1.687458000$

$\begin{array}{llll}\text { H } & 0.799985000 & -2.569397000 & 2.114644000\end{array}$

C $\quad-1.792777000 \quad-1.073827000 \quad 0.635586000$

$\begin{array}{llll}\text { C } & -2.916388000 & -0.337437000 & 1.188181000\end{array}$

$\begin{array}{llll}\text { C } & -4.182476000 & -0.330479000 & 0.559784000\end{array}$

$\begin{array}{llll}\text { H } & -4.300147000 & -0.859451000 & -0.394465000\end{array}$

$\begin{array}{llll}\text { C } & -5.271067000 & 0.330789000 & 1.142369000\end{array}$

$\begin{array}{llll}\text { H } & -6.247955000 & 0.330382000 & 0.644311000\end{array}$

$\begin{array}{llll}\text { C } & -5.102660000 & 0.983049000 & 2.384245000\end{array}$

$\begin{array}{llll}\text { H } & -5.951319000 & 1.496842000 & 2.853306000\end{array}$

$\begin{array}{llll}\text { C } & -3.854460000 & 0.991352000 & 3.022643000\end{array}$

H $\quad-3.702384000 \quad 1.514824000 \quad 3.973690000$

$\begin{array}{llll}\text { C } & -2.747193000 & 0.357375000 & 2.420239000\end{array}$

H $\quad-2.015461000 \quad-1.852302000-0.105772000$

$\begin{array}{llll}\text { H } & -0.101997000 & -0.015485000 & -2.186680000\end{array}$

$\begin{array}{llll}\text { B } & -0.326524000 & -0.243089000 & 2.555154000\end{array}$

$\begin{array}{llll}\text { O } & 0.613193000 & 0.689051000 & 1.825321000\end{array}$

$\begin{array}{llll}\text { O } & 0.426198000 & -0.793497000 & 3.646574000\end{array}$

$\begin{array}{llll}\text { C } & 1.942255000 & 0.608083000 & 2.487585000\end{array}$

C $\quad 1.504717000 \quad 0.141589000 \quad 3.929211000$

$\begin{array}{llll}\text { C } & 0.951448000 & 1.308895000 & 4.769120000\end{array}$

$\begin{array}{llll}\text { C } & 2.591802000 & -0.602543000 & 4.705533000\end{array}$

$\begin{array}{llll}\text { C } & 2.599970000 & 1.986544000 & 2.441280000\end{array}$

$\begin{array}{llll}\text { C } & 2.858529000 & -0.404027000 & 1.801258000\end{array}$

$\begin{array}{llll}\text { H } & 2.884086000 & 2.230780000 & 1.402793000\end{array}$

$\begin{array}{llll}\text { H } & 1.928868000 & 2.773381000 & 2.813642000\end{array}$

$\begin{array}{llll}\text { H } & 2.998858000 & -0.118908000 & 0.749759000\end{array}$

$\begin{array}{llll}H & 1.762144000 & 1.976527000 & 5.109801000\end{array}$

$\begin{array}{llll}H & 0.218573000 & 1.901692000 & 4.195618000\end{array}$

$\begin{array}{llll}H & 2.455309000 & -1.425953000 & 1.837007000\end{array}$

$\begin{array}{llll}\text { H } & 3.848705000 & -0.399771000 & 2.287555000\end{array}$

$\begin{array}{llll}H & 3.517495000 & 1.977967000 & 3.057075000\end{array}$

$\begin{array}{llll}\text { H } & 0.445638000 & 0.896778000 & 5.659550000\end{array}$

$\begin{array}{llll}H & 3.491921000 & 0.027610000 & 4.819306000\end{array}$

$\begin{array}{llll}H & 2.875116000 & -1.540170000 & 4.200275000\end{array}$

$\begin{array}{llll}\text { H } & 2.219308000 & -0.853082000 & 5.714519000\end{array}$

$\begin{array}{llll}\text { C } & -1.160660000 & -1.810996000 & -2.853843000\end{array}$

$\begin{array}{llll}\text { C } & -2.319806000 & -2.623687000 & -2.727890000\end{array}$

H $\quad-3.205983000 \quad-2.226303000 \quad-2.216014000$

$\begin{array}{llll}\text { C } & -2.338505000 & -3.935668000 & -3.225651000\end{array}$

H $\quad-3.242575000 \quad-4.547265000 \quad-3.112035000$

C $\quad-1.195931000-4.474149000 \quad-3.849022000$

H $\quad-1.206142000 \quad-5.505421000 \quad-4.223376000$

C $\quad-0.039590000 \quad-3.681258000 \quad-3.986227000$

H $\quad 0.852737000 \quad-4.090480000-4.476770000$

$\begin{array}{llll}\text { C } & -0.023722000 & -2.365982000 & -3.499562000\end{array}$

$\begin{array}{llll}\text { H } & 0.873827000 & -1.746862000 & -3.621673000\end{array}$

$\begin{array}{llll}\text { C } & -1.103784000 & -0.464473000 & -2.286007000\end{array}$

$\begin{array}{llll}\text { C } & -2.185844000 & 0.387565000 & -2.098104000\end{array}$

H $\quad-3.144578000 \quad 0.151559000 \quad-2.588336000$

$\begin{array}{llll}\text { C } & -1.217650000 & 2.751842000 & 0.679303000\end{array}$

$\begin{array}{llll}\text { C } & -0.013347000 & 3.423777000 & 1.002979000\end{array}$

$\begin{array}{llll}\text { H } & 0.916046000 & 3.025552000 & 0.588271000\end{array}$

$\begin{array}{llll}\text { C } & -0.021888000 & 4.574006000 & 1.804739000\end{array}$

$\begin{array}{llll}\text { H } & 0.922743000 & 5.085807000 & 2.032260000\end{array}$

$\begin{array}{llll}\text { C } & -1.234059000 & 5.068399000 & 2.326399000\end{array}$

$\begin{array}{llll}\text { H } & -1.237648000 & 5.957583000 & 2.970105000\end{array}$

$\begin{array}{llll}\text { C } & -2.441021000 & 4.416008000 & 2.010212000\end{array}$

$\begin{array}{llll}\text { H } & -3.392069000 & 4.791557000 & 2.410271000\end{array}$

$\begin{array}{llll}\text { C } & -2.434965000 & 3.281980000 & 1.183081000\end{array}$

$\begin{array}{llll}\text { H } & -3.375913000 & 2.771757000 & 0.946469000\end{array}$

Page 884 
C $\quad-1.215030000 \quad 1.561861000 \quad-0.184946000$

C $\quad-2.112322000 \quad 1.528698000 \quad-1.243342000$

$\begin{array}{llll}\text { H } & -2.827688000 & 2.352923000 & -1.402373000\end{array}$

\section{${ }^{1}$ 5IV-ABB $\quad$ FSPE: $-\mathbf{3 1 8 5 . 7 6 6 8 2 6 9 3 3 5 9 5} E_{\mathrm{h}}$}

Fe $\quad 0.058608000 \quad-0.128767000 \quad 0.025224000$

$\begin{array}{llll}\mathrm{O} & -1.574048000 & 0.355570000 & 2.973786000\end{array}$

C $\quad 2.209633000 \quad-1.737656000 \quad-1.249964000$

H $\quad 2.652053000 \quad-2.718077000 \quad-1.499060000$

$\begin{array}{llll}\text { O } & 1.279099000 & 1.058084000 & -0.868215000\end{array}$

C $\quad 2.829156000 \quad-0.576830000 \quad-1.841195000$

N $\quad-0.583853000 \quad-1.387186000 \quad 1.455792000$

$\begin{array}{llll}\text { C } & 3.977830000 & -0.762129000 & -2.662775000\end{array}$

H $\quad 4.313886000 \quad-1.789584000 \quad-2.859698000$

N $\quad 1.196646000 \quad-1.695437000 \quad-0.432503000$

C $\quad 4.677325000 \quad 0.318289000 \quad-3.199647000$

$\begin{array}{llll}\text { H } & 5.557690000 & 0.154189000 & -3.832061000\end{array}$

$\begin{array}{llll}\text { C } & 4.237635000 & 1.632870000 & -2.903156000\end{array}$

$\begin{array}{llll}\mathrm{H} & 4.782089000 & 2.495900000 & -3.308706000\end{array}$

$\begin{array}{llll}\text { C } & 3.114546000 & 1.849139000 & -2.102676000\end{array}$

$\begin{array}{llll}\text { H } & 2.768357000 & 2.864962000 & -1.874332000\end{array}$

C $\quad 2.358918000 \quad 0.759691000 \quad-1.571071000$

$\begin{array}{llll}\text { C } & 0.666643000 & -2.969560000 & 0.079135000\end{array}$

H $\quad 1.430163000 \quad-3.768159000 \quad 0.092532000$

$\begin{array}{llll}H & -0.148843000 & -3.298242000 & -0.596026000\end{array}$

C $\quad 0.150780000 \quad-2.678483000 \quad 1.478011000$

H $\quad-0.522797000 \quad-3.468813000 \quad 1.853318000$

$\begin{array}{llll}\text { H } & 0.980750000 & -2.557005000 & 2.190109000\end{array}$

$\begin{array}{llll}\text { C } & -1.706106000 & -1.295115000 & 0.665521000\end{array}$

$\begin{array}{llll}\text { C } & -2.855457000 & -0.539846000 & 1.118972000\end{array}$

$\begin{array}{llll}\text { C } & -4.086828000 & -0.579846000 & 0.426688000\end{array}$

H $\quad-4.177365000 \quad-1.230284000 \quad-0.451694000$

$\begin{array}{llll}\text { C } & -5.171138000 & 0.201921000 & 0.842218000\end{array}$

H $\quad-6.116444000 \quad 0.172743000 \quad 0.288243000$

$\begin{array}{llll}\text { C } & -5.030788000 & 1.037291000 & 1.970303000\end{array}$

$\begin{array}{llll}\text { H } & -5.870422000 & 1.664318000 & 2.295649000\end{array}$

C $\quad-3.823797000 \quad 1.077925000 \quad 2.684961000$

$\begin{array}{llll}\text { H } & -3.703968000 & 1.720175000 & 3.566008000\end{array}$

$\begin{array}{llll}\text { C } & -2.727930000 & 0.295986000 & 2.266470000\end{array}$

H $\quad-1.876832000 \quad-2.114013000 \quad-0.045444000$

$\begin{array}{llll}\text { H } & -0.130402000 & -0.086689000 & -2.055645000\end{array}$

$\begin{array}{llll}\text { B } & -0.313502000 & -0.287455000 & 2.565642000\end{array}$

$\begin{array}{llll}\text { O } & 0.587203000 & 0.653931000 & 1.806269000\end{array}$

$\begin{array}{llll}0 & 0.451042000 & -0.736282000 & 3.692090000\end{array}$

$\begin{array}{llll}\text { C } & 1.905165000 & 0.700698000 & 2.472819000\end{array}$

$\begin{array}{llll}\text { C } & 1.495987000 & 0.253722000 & 3.934213000\end{array}$

$\begin{array}{llll}\text { C } & 0.901394000 & 1.412701000 & 4.754168000\end{array}$

$\begin{array}{llll}\text { C } & 2.621336000 & -0.422218000 & 4.718623000\end{array}$

$\begin{array}{llll}\text { C } & 2.411264000 & 2.137157000 & 2.339990000\end{array}$

$\begin{array}{llll}\text { C } & 2.901385000 & -0.260861000 & 1.825125000\end{array}$

$\begin{array}{llll}H & 2.651367000 & 2.336871000 & 1.281070000\end{array}$

$\begin{array}{llll}\text { H } & 1.648496000 & 2.863122000 & 2.662955000\end{array}$

$\begin{array}{llll}H & 3.015033000 & -0.011866000 & 0.761019000\end{array}$

$\begin{array}{llll}H & 1.678489000 & 2.143486000 & 5.038671000\end{array}$

$\begin{array}{llll}H & 0.108836000 & 1.936180000 & 4.193557000\end{array}$

$\begin{array}{llll}H & 2.578205000 & -1.309193000 & 1.903548000\end{array}$

$\begin{array}{llll}\text { H } & 3.888879000 & -0.159665000 & 2.306239000\end{array}$

$\begin{array}{llll}H & 3.325015000 & 2.284144000 & 2.943415000\end{array}$

H $\quad 0.457296000 \quad 1.003155000 \quad 5.678269000$

$\begin{array}{llll}H & 3.490932000 & 0.252546000 & 4.812071000\end{array}$

H $\quad 2.946997000 \quad-1.356013000 \quad 4.232332000$

$\begin{array}{llll}\text { H } & 2.267775000 & -0.667995000 & 5.735563000\end{array}$

$\begin{array}{lrrr}\text { C } & -1.267002000 & -1.793641000 & -2.804082000\end{array}$ 


$\begin{array}{lrrr}\text { C } & -2.468599000 & -2.549509000 & -2.758759000 \\ \text { H } & -3.353923000 & -2.127430000 & -2.267053000 \\ \text { C } & -2.534099000 & -3.835755000 & -3.316330000 \\ \text { H } & -3.472635000 & -4.402305000 & -3.265656000 \\ \text { C } & -1.396771000 & -4.406567000 & -3.920404000 \\ \text { H } & -1.444912000 & -5.418145000 & -4.342607000 \\ \text { C } & -0.196582000 & -3.671088000 & -3.974982000 \\ \text { H } & 0.693689000 & -4.104513000 & -4.448241000 \\ \text { C } & -0.134139000 & -2.381010000 & -3.427898000 \\ \text { H } & 0.798471000 & -1.806027000 & -3.486803000 \\ \text { C } & -1.159514000 & -0.470976000 & -2.184100000 \\ \text { C } & -2.191967000 & 0.430552000 & -1.971624000 \\ \text { H } & -3.156677000 & 0.247232000 & -2.468191000 \\ \text { C } & -3.003585000 & 2.692684000 & -1.084525000 \\ \text { C } & -2.582655000 & 3.974920000 & -0.651423000 \\ \text { H } & -1.540492000 & 4.118679000 & -0.337474000 \\ \text { C } & -3.468220000 & 5.065211000 & -0.632573000 \\ \text { H } & -3.112250000 & 6.047523000 & -0.295002000 \\ \text { C } & -4.801196000 & 4.904559000 & -1.055688000 \\ \text { H } & -5.494026000 & 5.755674000 & -1.044724000 \\ \text { C } & -5.232689000 & 3.640283000 & -1.499146000 \\ \text { H } & -6.270649000 & 3.497419000 & -1.827412000 \\ \text { C } & -4.346002000 & 2.551314000 & -1.516030000 \\ \text { H } & -4.715817000 & 1.570397000 & -1.836465000 \\ \text { C } & -1.130539000 & 1.366936000 & -0.039721000 \\ \text { C } & -2.064527000 & 1.536668000 & -1.049518000 \\ \text { H } & -1.072903000 & 2.120043000 & 0.766196000\end{array}$

\section{${ }^{15 V-A A A A} \quad$ FSPE: $-3494.204685741256 E_{\mathrm{h}}$}

$\begin{array}{lrrr}\text { Fe } & -0.372773000 & 0.424336000 & -0.334312000 \\ \text { O } & -1.421122000 & 1.399135000 & 3.333868000 \\ \text { C } & 2.048891000 & -1.094907000 & -0.841785000 \\ \text { H } & 2.710098000 & -1.961415000 & -0.670838000 \\ \text { O } & 0.750942000 & 1.458900000 & -1.374564000 \\ \text { C } & 2.457171000 & -0.188080000 & -1.874380000 \\ \text { N } & -0.389741000 & -0.776467000 & 2.672523000 \\ \text { C } & 3.596415000 & -0.504966000 & -2.672575000 \\ \text { H } & 4.107809000 & -1.461649000 & -2.497456000 \\ \text { N } & 0.986286000 & -0.977140000 & -0.076762000 \\ \text { C } & 4.059620000 & 0.367209000 & -3.655114000 \\ \text { H } & 4.930071000 & 0.102144000 & -4.267160000 \\ \text { C } & 3.392924000 & 1.607440000 & -3.855397000 \\ \text { H } & 3.748490000 & 2.298604000 & -4.631022000 \\ \text { C } & 2.283173000 & 1.953026000 & -3.087641000 \\ \text { H } & 1.747219000 & 2.895775000 & -3.245091000 \\ \text { C } & 1.783854000 & 1.067719000 & -2.085030000 \\ \text { C } & 0.796335000 & -2.067079000 & 0.899201000 \\ \text { H } & 1.600482000 & -2.816803000 & 0.784403000 \\ \text { H } & -0.152622000 & -2.577237000 & 0.660637000 \\ \text { C } & 0.769316000 & -1.630226000 & 2.364771000 \\ \text { H } & 0.726691000 & -2.537458000 & 2.993706000 \\ \text { H } & 1.672718000 & -1.069866000 & 2.639043000 \\ \text { C } & -1.588516000 & -1.306451000 & 2.633194000 \\ \text { C } & -2.769036000 & -0.542089000 & 2.864459000 \\ \text { C } & -4.063603000 & -1.117894000 & 2.747723000 \\ \text { H } & -4.149251000 & -2.173279000 & 2.458173000 \\ \text { C } & -5.199781000 & -0.351967000 & 3.000429000 \\ \text { H } & -6.199631000 & -0.789874000 & 2.903050000 \\ \text { C } & -5.047834000 & 0.999872000 & 3.403728000 \\ \text { H } & -5.938971000 & 1.600774000 & 3.625535000 \\ \text { C } & -3.784885000 & 1.586051000 & 3.531485000 \\ \text { H } & -3.674665000 & 2.624606000 & 3.864558000 \\ \text { C } & -2.621004000 & 0.832129000 & 3.235650000\end{array}$


$\begin{array}{llll}\text { H } & -1.679171000 & -2.377152000 & 2.398689000\end{array}$

$\begin{array}{llll}\text { B } & -0.104647000 & 0.747024000 & 2.974303000\end{array}$

$\begin{array}{llll}\text { O } & 0.495383000 & 1.374163000 & 1.803659000\end{array}$

$\begin{array}{llll}\text { O } & 0.845202000 & 0.884060000 & 4.054050000\end{array}$

$\begin{array}{llll}\text { C } & 1.762752000 & 2.007013000 & 2.164354000\end{array}$

$\begin{array}{llll}\text { C } & 1.675168000 & 2.041599000 & 3.744423000\end{array}$

$\begin{array}{llll}\text { C } & 0.957503000 & 3.302412000 & 4.263076000\end{array}$

$\begin{array}{llll}\text { C } & 3.020245000 & 1.873415000 & 4.453546000\end{array}$

C $\quad 1.827464000 \quad 3.386116000 \quad 1.505804000$

$\begin{array}{llll}\text { C } & 2.926831000 & 1.162850000 & 1.627205000\end{array}$

$\begin{array}{llll}\text { H } & 1.834909000 & 3.259419000 & 0.410045000\end{array}$

$\begin{array}{llll}H & 0.956255000 & 4.005151000 & 1.762955000\end{array}$

$\begin{array}{llll}H & 2.800720000 & 1.043502000 & 0.539905000\end{array}$

$\begin{array}{llll}H & 1.586717000 & 4.202442000 & 4.146994000\end{array}$

$\begin{array}{llll}H & 0.005201000 & 3.466132000 & 3.731237000\end{array}$

$\begin{array}{llll}\text { H } & 2.966675000 & 0.159636000 & 2.078955000\end{array}$

$\begin{array}{llll}H & 3.893340000 & 1.663137000 & 1.812085000\end{array}$

$\begin{array}{llll}H & 2.748764000 & 3.914830000 & 1.811226000\end{array}$

$\begin{array}{llll}H & 0.738508000 & 3.169372000 & 5.337386000\end{array}$

$\begin{array}{llll}H & 3.719858000 & 2.675725000 & 4.157704000\end{array}$

H $\quad 3.479771000 \quad 0.899622000 \quad 4.216846000$

$\begin{array}{llll}\text { H } & 2.876620000 & 1.930803000 & 5.547449000\end{array}$

C $\quad-1.391501000 \quad-2.012951000 \quad-1.418050000$

C $\quad-2.390257000 \quad-2.313144000 \quad-0.455017000$

$\begin{array}{llll}\text { C } & -0.647843000 & -3.097349000 & -1.955078000\end{array}$

H $\quad-2.968405000 \quad-1.497185000 \quad-0.009282000$

$\begin{array}{llll}H & 0.149594000 & -2.880650000 & -2.676763000\end{array}$

$\begin{array}{llll}\text { C } & -2.633556000 & -3.636228000 & -0.047275000\end{array}$

C $\quad-0.880622000 \quad-4.417346000 \quad-1.536014000$

H $\quad-3.415891000 \quad-3.839828000 \quad 0.695655000$

H $\quad-0.275355000 \quad-5.233218000 \quad-1.952279000$

C $\quad-1.874562000 \quad-4.696090000 \quad-0.577712000$

H $\quad-2.052819000 \quad-5.726576000 \quad-0.246082000$

C $\quad-1.059261000-0.620024000 \quad-1.821425000$

C $\quad-1.115496000 \quad-0.325957000 \quad-3.166327000$

H $\quad-1.332491000 \quad-1.194724000 \quad-3.815978000$

$\begin{array}{llll}\text { C } & -0.908111000 & 0.903421000 & -3.912849000\end{array}$

$\begin{array}{llll}\text { C } & -1.171792000 & 2.181540000 & -3.506147000\end{array}$

C $\quad-0.894025000 \quad 3.408168000 \quad-4.253427000$

C $\quad-1.474970000 \quad 4.6350000000 \quad-3.828615000$

$\begin{array}{llll}\text { C } & -0.028368000 & 3.450098000 & -5.382654000\end{array}$

$\begin{array}{llll}\text { H } & -2.130893000 & 4.634678000 & -2.948325000\end{array}$

$\begin{array}{llll}H & 0.476724000 & 2.533068000 & -5.710802000\end{array}$

C $\quad-1.218227000 \quad 5.839095000-4.502699000$

$\begin{array}{llll}\text { C } & 0.222612000 & 4.652005000 & -6.060079000\end{array}$

$\begin{array}{llll}\text { H } & -1.679717000 & 6.769610000 & -4.146978000\end{array}$

$\begin{array}{llll}H & 0.899197000 & 4.654767000 & -6.924890000\end{array}$

$\begin{array}{llll}\text { C } & -0.369705000 & 5.856251000 & -5.626460000\end{array}$

H $\quad-0.162732000 \quad 6.796876000 \quad-6.152390000$

H $\quad-0.551084000 \quad 0.753645000 \quad-4.945105000$

$\begin{array}{llll}\text { H } & -1.631142000 & 2.314573000 & -2.519073000\end{array}$

$\begin{array}{llll}\text { C } & -1.356919000 & 3.370747000 & 0.342840000\end{array}$

$\begin{array}{llll}\text { C } & -0.590270000 & 4.139458000 & -0.571960000\end{array}$

H $\quad-0.145245000 \quad 3.636839000 \quad-1.435096000$

$\begin{array}{llll}\text { C } & -0.377406000 & 5.506242000 & -0.346952000\end{array}$

$\begin{array}{llll}\text { H } & 0.204874000 & 6.086546000 & -1.073869000\end{array}$

C $\quad-0.885730000 \quad 6.125648000 \quad 0.811004000$

$\begin{array}{llll}\text { H } & -0.701482000 & 7.191656000 & 0.994907000\end{array}$

$\begin{array}{llll}\text { C } & -1.629715000 & 5.365348000 & 1.735002000\end{array}$

$\begin{array}{llll}\text { H } & -2.025739000 & 5.836791000 & 2.643506000\end{array}$

C $\quad-1.875363000 \quad 4.004352000 \quad 1.500019000$

$\begin{array}{llll}\text { H } & -2.452260000 & 3.414310000 & 2.214599000\end{array}$

$\begin{array}{llll}\text { C } & -1.595039000 & 1.977176000 & 0.065365000\end{array}$

$\begin{array}{llll}\text { C } & -2.254869000 & 0.942054000 & -0.319119000\end{array}$

H $\quad-3.1972300000 .598327000 \quad-0.749545000$ 


\section{${ }^{15 V-A A B A} \quad$ FSPE: $-3494.192189760136 E_{\mathrm{h}}$}

$\begin{array}{llll}\mathrm{Fe} & -0.169662000 & 0.391185000 & 0.058002000\end{array}$

\begin{tabular}{llll}
\hline & -1.575241000 & 1.524929000 & 3.074802000
\end{tabular}

$\begin{array}{llll}\text { C } & 2.192581000 & -1.071805000 & -0.786003000\end{array}$

$\begin{array}{llll}\text { H } & 2.822736000 & -1.974250000 & -0.724065000\end{array}$

$\begin{array}{llll}\text { O } & 0.824453000 & 1.484786000 & -1.086210000\end{array}$

$\begin{array}{llll}\text { C } & 2.578275000 & -0.068260000 & -1.739609000\end{array}$

$\begin{array}{llll}\mathrm{N} & -0.544069000 & -0.447313000 & 1.899233000\end{array}$

$\begin{array}{llll}\text { C } & 3.707574000 & -0.296405000 & -2.574932000\end{array}$

H $\quad 4.243756000 \quad-1.250541000 \quad-2.480824000$

$\begin{array}{llll}\mathrm{N} & 1.174408000 & -0.985423000 & 0.024967000\end{array}$

$\begin{array}{llll}\text { C } & 4.133645000 & 0.660375000 & -3.497008000\end{array}$

H $\quad 5.001272000 \quad 0.467072000 \quad-4.138744000$

$\begin{array}{llll}\text { C } & 3.429174000 & 1.886544000 & -3.592536000\end{array}$

H $\quad 3.750431000 \quad 2.645890000 \quad-4.317431000$

$\begin{array}{llll}\text { C } & 2.322695000 & 2.142449000 & -2.779841000\end{array}$

$\begin{array}{llll}\text { H } & 1.773518000 & 3.087690000 & -2.860192000\end{array}$

C $\quad 1.864159000 \quad 1.175933000 \quad-1.836262000$

$\begin{array}{llll}\text { C } & 0.867896000 & -2.152333000 & 0.867440000\end{array}$

$\begin{array}{llll}\text { H } & 1.754205000 & -2.792875000 & 1.020164000\end{array}$

$\begin{array}{llll}\text { H } & 0.103608000 & -2.760356000 & 0.342864000\end{array}$

$\begin{array}{llll}\text { C } & 0.337934000 & -1.614762000 & 2.178762000\end{array}$

$\begin{array}{llll}\text { H } & -0.232519000 & -2.370017000 & 2.745911000\end{array}$

$\begin{array}{llll}\text { H } & 1.152609000 & -1.244523000 & 2.816753000\end{array}$

C $\quad-1.750709000 \quad-0.686471000 \quad 1.302844000$

$\begin{array}{llll}\text { C } & -2.921477000 & 0.070163000 & 1.682317000\end{array}$

$\begin{array}{llll}\text { C } & -4.202606000 & -0.250885000 & 1.172256000\end{array}$

H $\quad-4.294523000 \quad-1.090165000 \quad 0.469812000$

$\begin{array}{llll}\text { C } & -5.328713000 & 0.490090000 & 1.548946000\end{array}$

$\begin{array}{llll}\text { H } & -6.317451000 & 0.236291000 & 1.148683000\end{array}$

$\begin{array}{llll}\text { C } & -5.180068000 & 1.562823000 & 2.457529000\end{array}$

$\begin{array}{llll}\text { H } & -6.058020000 & 2.146184000 & 2.763106000\end{array}$

$\begin{array}{llll}\text { C } & -3.920345000 & 1.894346000 & 2.977657000\end{array}$

$\begin{array}{llll}\text { H } & -3.796377000 & 2.724639000 & 3.683764000\end{array}$

C $\quad-2.776025000 \quad 1.164191000 \quad 2.586848000$

$\begin{array}{llll}\text { H } & -1.904068000 & -1.679190000 & 0.862589000\end{array}$

$\begin{array}{llll}\text { B } & -0.274872000 & 0.880581000 & 2.743273000\end{array}$

$\begin{array}{llll}\text { O } & 0.558678000 & 1.689342000 & 1.839191000\end{array}$

$\begin{array}{llll}\text { O } & 0.502238000 & 0.651613000 & 3.935993000\end{array}$

$\begin{array}{llll}\text { C } & 1.854590000 & 1.941109000 & 2.466746000\end{array}$

C $\quad 1.518647000 \quad 1.694375000 \quad 3.990508000$

$\begin{array}{llll}\text { C } & 0.898849000 & 2.937274000 & 4.655817000\end{array}$

C $\quad 2.697253000 \quad 1.179494000 \quad 4.817860000$

$\begin{array}{llll}\text { C } & 2.282791000 & 3.372104000 & 2.138253000\end{array}$

$\begin{array}{llll}\text { C } & 2.899061000 & 0.969072000 & 1.905981000\end{array}$

$\begin{array}{llll}\text { H } & 2.497237000 & 3.453368000 & 1.059352000\end{array}$

$\begin{array}{llll}H & 1.491022000 & 4.097074000 & 2.376180000\end{array}$

$\begin{array}{llll}H & 2.916104000 & 1.066114000 & 0.808567000\end{array}$

$\begin{array}{llll}H & 1.650398000 & 3.730483000 & 4.814525000\end{array}$

$\begin{array}{llll}H & 0.077134000 & 3.345219000 & 4.042935000\end{array}$

$\begin{array}{llll}\text { H } & 2.669072000 & -0.078315000 & 2.152358000\end{array}$

$\begin{array}{llll}H & 3.905265000 & 1.206530000 & 2.292676000\end{array}$

$\begin{array}{llll}H & 3.196871000 & 3.638529000 & 2.699382000\end{array}$

$\begin{array}{llll}H & 0.488527000 & 2.647088000 & 5.639118000\end{array}$

$\begin{array}{llll}H & 3.538524000 & 1.894908000 & 4.783126000\end{array}$

$\begin{array}{llll}\text { H } & 3.049578000 & 0.202302000 & 4.448420000\end{array}$

$\begin{array}{llll}\text { H } & 2.392216000 & 1.060650000 & 5.872883000\end{array}$

$\begin{array}{llll}\text { C } & -1.869870000 & -1.871897000 & -3.284038000\end{array}$

$\begin{array}{llll}\text { C } & -2.636182000 & -2.834264000 & -2.580065000\end{array}$

C $\quad-1.517401000 \quad-2.163686000 \quad-4.625762000$

H $\quad-2.952659000 \quad-2.615287000 \quad-1.551512000$

H $\quad-0.920470000 \quad-1.437436000 \quad-5.193642000$ 


$\begin{array}{llll}\text { C } & -3.016836000 & -4.045748000 & -3.182122000 \\ \text { C } & -1.898002000 & -3.373758000 & -5.231777000 \\ \text { H } & -3.616362000 & -4.769619000 & -2.614678000 \\ \text { H } & -1.601799000 & -3.578518000 & -6.269019000 \\ \text { C } & -2.648174000 & -4.323239000 & -4.512239000 \\ \text { H } & -2.947514000 & -5.267187000 & -4.985300000 \\ \text { C } & -0.915809000 & -0.701896000 & -1.361715000 \\ \text { C } & -1.439237000 & -0.581742000 & -2.635236000 \\ \text { H } & -0.862762000 & -1.763705000 & -1.063485000 \\ \text { C } & -1.661072000 & 0.597548000 & -3.481600000 \\ \text { C } & -1.182138000 & 1.871378000 & -3.361797000 \\ \text { C } & -1.493195000 & 2.995877000 & -4.249980000 \\ \text { C } & -1.037083000 & 4.295321000 & -3.896504000 \\ \text { C } & -2.226397000 & 2.871268000 & -5.463147000 \\ \text { H } & -0.477527000 & 4.423998000 & -2.961744000 \\ \text { H } & -2.589066000 & 1.886389000 & -5.783763000 \\ \text { C } & -1.299985000 & 5.413697000 & -4.703493000 \\ \text { C } & -2.487948000 & 3.986627000 & -6.271291000 \\ \text { H } & -0.935440000 & 6.402880000 & -4.397274000 \\ \text { H } & -3.053284000 & 3.858082000 & -7.203651000 \\ \text { C } & -2.028199000 & 5.267185000 & -5.898830000 \\ \text { H } & -2.234714000 & 6.137267000 & -6.534712000 \\ \text { H } & -2.326219000 & 0.392834000 & -4.335275000 \\ \text { H } & -0.519810000 & 2.094334000 & -2.519261000 \\ \text { C } & -0.811336000 & 3.857038000 & 0.534884000 \\ \text { C } & 0.171673000 & 4.521180000 & -0.244440000 \\ \text { H } & 0.563487000 & 4.011712000 & -1.129408000 \\ \text { C } & 0.659792000 & 5.773785000 & 0.148966000 \\ \text { H } & 1.424158000 & 6.274874000 & -0.457643000 \\ \text { C } & 0.181210000 & 6.380066000 & 1.326989000 \\ \text { H } & 0.565410000 & 7.360372000 & 1.635378000 \\ \text { C } & -0.783919000 & 5.721887000 & 2.115574000 \\ \text { H } & -1.147739000 & 6.186799000 & 3.040179000 \\ \text { C } & -1.277932000 & 4.468421000 & 1.728723000 \\ \text { H } & -2.017760000 & 3.942977000 & 2.340361000 \\ \text { C } & -1.366440000 & 2.624648000 & 0.091505000 \\ \text { C } & -1.906729000 & 1.599073000 & -0.372731000 \\ \text { H } & -2.732388000 & 1.118464000 & -0.883591000 \\ & & & \end{array}$

\section{${ }^{15 V-A B A A} \quad$ FSPE: $-3494.154094632398 E_{\mathrm{h}}$}

$\begin{array}{lrrr}\text { Fe } & -0.310506000 & -0.316461000 & 0.050502000 \\ \text { O } & -1.956748000 & 0.750444000 & 3.058917000 \\ \text { C } & 1.669942000 & -2.535625000 & -0.360270000 \\ \text { H } & 1.918690000 & -3.610369000 & -0.330075000 \\ \text { O } & 1.322903000 & 0.389362000 & -0.646714000 \\ \text { C } & 2.666933000 & -1.647416000 & -0.901176000 \\ \text { N } & -1.378919000 & -1.200676000 & 1.590575000 \\ \text { C } & 3.891139000 & -2.195351000 & -1.368981000 \\ \text { H } & 4.040409000 & -3.280320000 & -1.283360000 \\ \text { N } & 0.511008000 & -2.153704000 & 0.084007000 \\ \text { C } & 4.878564000 & -1.395205000 & -1.944943000 \\ \text { H } & 5.811821000 & -1.837633000 & -2.312580000 \\ \text { C } & 4.652794000 & -0.000352000 & -2.047471000 \\ \text { H } & 5.417561000 & 0.645812000 & -2.498396000 \\ \text { C } & 3.462539000 & 0.568794000 & -1.588853000 \\ \text { H } & 3.280874000 & 1.647341000 & -1.670373000 \\ \text { C } & 2.426442000 & -0.231966000 & -1.015276000 \\ \text { C } & -0.440844000 & -3.180983000 & 0.515478000 \\ \text { H } & 0.040375000 & -4.161532000 & 0.684207000 \\ \text { H } & -1.192980000 & -3.307426000 & -0.286696000 \\ \text { C } & -1.089943000 & -2.652049000 & 1.775048000 \\ \text { H } & -2.027408000 & -3.176867000 & 2.026288000 \\ \text { H } & -0.405930000 & -2.723682000 & 2.633104000\end{array}$


C -2.413665000

C -3.334368000

C -4.517315000

H $\quad-4.679997000$

C $\quad-5.480666000$

H $\quad-6.394890000$

C $\quad-5.282264000$

H $\quad-6.045511000$

C -4.113413000

H -3.929050000

C $\quad-3.103677000$

H $\quad-2.795466000$

H 1.030081000

B $\quad-0.879047000$

O 0.343999000

O -0.462355000

C $\quad 1.490071000$

C $\quad 0.752219000$

C $\quad 0.377590000$

C 1.501907000

C 2.460457000

C 2.244829000

H 2.902729000

H 1.971440000

H 2.608001000

H 1.264854000

H $\quad-0.084996000$

H 1.611667000

H 3.122233000

H 3.280361000

H $\quad-0.348157000$

H 2.500620000

H 1.622894000

H $\quad 0.936540000$

C $\quad 0.642707000$

C -0.301034000

H $\quad-1.348158000$

C $\quad 0.078563000$

H $\quad-0.671522000$

C 1.409273000

H 1.700008000

C 2.357683000

H 3.396653000

C $\quad 1.979141000$

H 2.725606000

C $\quad 0.303226000$

C -0.798897000

H $\quad-1.497061000$

C -1.022295000

C 0.131841000

H $\quad 1.078989000$

C $\quad 0.070791000$

H 0.984331000

C -1.154482000

H $\quad-1.207279000$

C -2.302190000

H $\quad-3.263972000$

C $\quad-2.233336000$

H -3.133341000

C -0.889723000

C -1.069072000

H -1.457402000

C -0.075447000

C $\quad 1.132504000$

H 1.518965000

C $\quad 1.830277000$ $\begin{array}{ll}-0.844287000 & 0.773740000\end{array}$

$0.201524000 \quad 1.154591000$

$0.455831000 \quad 0.418070000$

$-0.080004000-0.526567000$

$1.346900000 \quad 0.904779000$

$\begin{array}{ll}1.539888000 & 0.330978000\end{array}$

$1.965254000 \quad 2.162090000$

$2.644269000 \quad 2.562979000$

$1.735454000 \quad 2.897332000$

$2.241522000 \quad 3.851623000$

$0.893728000 \quad 2.378829000$

$\begin{array}{ll}-1.629789000 & 0.109087000\end{array}$

$1.042785000-2.703143000$

$-0.216777000 \quad 2.732409000$

$0.360787000 \quad 2.120541000$

$-0.871035000 \quad 3.949201000$

$0.058567000 \quad 3.003846000$

$-0.192987000 \quad 4.373612000$

$1.126619000 \quad 5.077106000$

$-1.111820000 \quad 5.339969000$

$1.241504000 \quad 3.017452000$

$\begin{array}{ll}-1.180500000 & 2.521736000\end{array}$

$\begin{array}{ll}1.370854000 & 2.013767000\end{array}$

$2.180443000 \quad 3.310430000$

$-1.008625000 \quad 1.499173000$

$1.600137000 \quad 5.533430000$

$1.840388000 \quad 4.373873000$

$\begin{array}{ll}-2.079595000 & 2.523863000\end{array}$

$-1.367105000 \quad 3.164393000$

$1.037438000 \quad 3.729703000$

$0.907573000 \quad 5.879852000$

$-0.701919000 \quad 5.574543000$

$-2.123591000 \quad 4.919708000$

$-1.195935000 \quad 6.285212000$

$1.241109000-4.844830000$

$1.372285000-5.899360000$

$1.601818000-5.663133000$

$1.186639000-7.237152000$

$1.289027000-8.032452000$

$0.854537000-7.564907000$

$0.701518000-8.611955000$

$0.712082000-6.533837000$

$0.450353000-6.773094000$

$0.903406000-5.196135000$

$0.793160000-4.396212000$

$1.418280000-3.431394000$

$2.039374000-2.919376000$

$2.523195000-3.624374000$

$\begin{array}{ll}2.654167000 & 0.817306000\end{array}$

$3.067083000 \quad 1.522772000$

$\begin{array}{ll}2.586277000 & 1.269003000\end{array}$

$4.077489000 \quad 2.492673000$

$4.380483000 \quad 3.022291000$

$\begin{array}{ll}4.713493000 & 2.780047000\end{array}$

$5.497623000 \quad 3.546713000$

$\begin{array}{ll}4.345244000 & 2.057639000\end{array}$

$\begin{array}{ll}4.835162000 & 2.259242000\end{array}$

$3.335671000 \quad 1.081283000$

$3.055393000 \quad 0.519833000$

$1.677457000-0.304227000$

$2.322946000-1.511578000$

$3.357371000-1.413772000$

$-2.359114000-2.852821000$

$-2.048361000-3.536304000$

$-1.029354000-3.497588000$

$-3.040411000-4.233611000$

Page $\$ 90$ 


$\begin{array}{lrrr}\text { H } & 2.764239000 & -2.782870000 & -4.746976000 \\ \text { C } & 1.340482000 & -4.360223000 & -4.265945000 \\ \text { H } & 1.892225000 & -5.137784000 & -4.808791000 \\ \text { C } & -0.790590000 & -1.365198000 & -2.135202000 \\ \text { C } & -1.525416000 & -0.499412000 & -1.585220000 \\ \text { H } & -2.404654000 & 0.120143000 & -1.750532000 \\ \text { C } & -0.566778000 & -3.696180000 & -2.903274000 \\ \text { H } & -1.509334000 & -3.942339000 & -2.400416000 \\ \text { C } & 0.138179000 & -4.682646000 & -3.604681000 \\ \text { H } & -0.250384000 & -5.707891000 & -3.637515000\end{array}$

\section{${ }^{1}$ 5V-ABBA $\quad$ FSPE: $-3494.178775690738 E_{\mathrm{h}}$}

Fe $\quad-0.202783000 \quad-0.575164000 \quad 0.082482000$

\begin{tabular}{llll}
\hline & -1.559031000 & 0.936790000 & 3.072328000
\end{tabular}

$\begin{array}{llll}\text { C } & 1.965989000 & -2.631451000 & -0.257701000\end{array}$

H $\quad 2.371161000 \quad-3.637015000 \quad-0.051628000$

$\begin{array}{llll}\text { O } & 1.207170000 & 0.133967000 & -1.001482000\end{array}$

C $\quad 2.703338000 \quad-1.804919000 \quad-1.179284000$

$\begin{array}{llll}\mathrm{N} & -0.879610000 & -1.202869000 & 1.946757000\end{array}$

$\begin{array}{llll}\text { C } & 3.858578000 & -2.343935000 & -1.803257000\end{array}$

$\begin{array}{llll}\text { H } & 4.146536000 & -3.375300000 & -1.558838000\end{array}$

$\begin{array}{llll}\mathrm{N} & 0.863623000 & -2.270864000 & 0.325038000\end{array}$

$\begin{array}{llll}\text { C } & 4.606899000 & -1.604294000 & -2.720699000\end{array}$

H $\quad 5.486065000 \quad-2.043016000 \quad-3.206932000$

$\begin{array}{llll}\text { C } & 4.214609000 & -0.274636000 & -3.005283000\end{array}$

H $\quad 4.797962000 \quad 0.328845000 \quad-3.713347000$

$\begin{array}{llll}\text { C } & 3.087033000 & 0.286052000 & -2.399828000\end{array}$

H $\quad 2.786537000 \quad 1.318967000 \quad-2.613691000$

$\begin{array}{llll}\text { C } & 2.276484000 & -0.465952000 & -1.495466000\end{array}$

$\begin{array}{llll}\text { C } & 0.154869000 & -3.258187000 & 1.142817000\end{array}$

$\begin{array}{llll}\text { H } & 0.784978000 & -4.127169000 & 1.406947000\end{array}$

$\begin{array}{llll}\text { H } & -0.706622000 & -3.630735000 & 0.553808000\end{array}$

$\begin{array}{llll}\text { C } & -0.319631000 & -2.517272000 & 2.371366000\end{array}$

H $\quad-1.090235000 \quad-3.068550000 \quad 2.937077000$

$\begin{array}{llll}H & 0.522298000 & -2.293018000 & 3.042940000\end{array}$

C $\quad-2.060053000 \quad-1.184264000 \quad 1.259735000$

$\begin{array}{llll}\text { C } & -3.066915000 & -0.185970000 & 1.547426000\end{array}$

$\begin{array}{llll}\text { C } & -4.354643000 & -0.240015000 & 0.959885000\end{array}$

H $\quad-4.561054000 \quad-1.013515000 \quad 0.208510000$

$\begin{array}{llll}\text { C } & -5.357586000 & 0.654405000 & 1.352779000\end{array}$

$\begin{array}{llll}\text { H } & -6.352101000 & 0.604376000 & 0.894245000\end{array}$

$\begin{array}{llll}\text { C } & -5.083421000 & 1.608146000 & 2.359738000\end{array}$

$\begin{array}{llll}\text { H } & -5.867652000 & 2.304409000 & 2.682036000\end{array}$

$\begin{array}{llll}\text { C } & -3.810547000 & 1.689797000 & 2.941644000\end{array}$

H $\quad-3.579662000 \quad 2.442022000 \quad 3.705071000$

$\begin{array}{llll}\text { C } & -2.782552000 & 0.816427000 & 2.522180000\end{array}$

$\begin{array}{llll}\text { H } & -2.393406000 & -2.136856000 & 0.827848000\end{array}$

$\begin{array}{llll}H & 0.469232000 & 0.770278000 & -2.844150000\end{array}$

$\begin{array}{llll}\text { B } & -0.387088000 & 0.080827000 & 2.743791000\end{array}$

$\begin{array}{llll}\text { O } & 0.566380000 & 0.682122000 & 1.779291000\end{array}$

$\begin{array}{llll}0 & 0.360028000 & -0.190068000 & 3.942723000\end{array}$

C $\quad 1.875829000 \quad 0.825041000 \quad 2.426545000$

$\begin{array}{llll}\text { C } & 1.479204000 & 0.745052000 & 3.955197000\end{array}$

$\begin{array}{llll}\text { C } & 0.995814000 & 2.098924000 & 4.506927000\end{array}$

C $\quad 2.569410000 \quad 0.172803000 \quad 4.863380000$

$\begin{array}{llll}\text { C } & 2.461194000 & 2.162341000 & 1.968943000\end{array}$

$\begin{array}{llll}\text { C } & 2.805897000 & -0.315746000 & 2.012431000\end{array}$

$\begin{array}{llll}\text { H } & 2.687964000 & 2.103122000 & 0.889984000\end{array}$

$\begin{array}{llll}H & 1.747423000 & 2.986701000 & 2.124635000\end{array}$

$\begin{array}{llll}H & 2.904735000 & -0.322543000 & 0.918357000\end{array}$

$\begin{array}{llll}H & 1.836392000 & 2.805484000 & 4.622798000\end{array}$

$\begin{array}{llll}H & 0.236365000 & 2.554274000 & 3.850054000\end{array}$

$\begin{array}{llll}H & 2.416465000 & -1.294797000 & 2.330861000\end{array}$ 


\begin{tabular}{|c|c|c|c|}
\hline & & & \\
\hline & $336>$ & & \\
\hline & 0.539626000 & 1.937548000 & 370000 \\
\hline & 3.488988000 & 0.781778000 & 4.800259000 \\
\hline & 2.813532000 & -0.867398000 & 4.593429000 \\
\hline & 2.222775000 & 0.184608000 & 46000 \\
\hline & -0.295019000 & 1.060999000 & 19000 \\
\hline & 73000 & 70000 & 0000 \\
\hline & -2.4 & 90000 & \\
\hline & -1.330143000 & .057789000. & \\
\hline & -2.227002000 & .164024000 & -7 \\
\hline & -0.087941000 & 386000 & 125000 \\
\hline & -0.011924000 & 0.645611000 & -8.8 \\
\hline & 1.050439000 & 0.609741000 & -6.8 \\
\hline & 2.023118000 & 00 & -7 \\
\hline & 0.947276000 & 0.756054000 & 400 \\
\hline & 1.840076000 & 407000 & -4 \\
\hline & -0.355421000 & 08000 & -3 \\
\hline & -1.329833000 & 08000 & \\
\hline & -2.115567000 & & \\
\hline & -2.038528000 & 00 & -0. \\
\hline & -1.588709000 & 7000 & 9000 \\
\hline & -0.787787000 & 000 & 99000 \\
\hline & -2.137157000 & 5.7 & -1. \\
\hline & -1.7 & & \\
\hline & -3.158134000 & 5.8 & -0. \\
\hline & -3.586604000 & 58000 & 0. \\
\hline & -3.629852000 & 58000 & 000 \\
\hline & -4.43 & 00 & 000 \\
\hline & -3.0 & & \\
\hline & -3.46 & & 000 \\
\hline & -1.07 & & \\
\hline & -1.433379000 & & -1. \\
\hline & -1.259352000 & 251000 & 667000 \\
\hline & -0.01 & & -2 \\
\hline & 0.731909000 & 45000 & 000 \\
\hline & 0.642273000 & 85000 & 35000 \\
\hline & 1.587890000 & 28000 & 9000 \\
\hline & 2.167943000 & 76000 & -5 \\
\hline & 1.721 & -4.4 & -3. \\
\hline & 2.4078 & 41000 & -4 \\
\hline & -0.812138000 & 088000 & 90300 \\
\hline & -1.613211000 & -0.984646000 & -1.319986000 \\
\hline & -2.591938000 & -0.520226000 & -1.420562000 \\
\hline & 0.102929000 & 08000 & 35000 \\
\hline & -0.482 & 44000 & -1 \\
\hline & & & \\
\hline & 1.061663000 & -6.015464000 & 26258030 \\
\hline
\end{tabular}

\section{${ }^{1}$ 5VI-AAAA $\quad$ FSPE: $-3494.248077529709 E_{\mathrm{h}}$}

$\begin{array}{lrrr}\text { Fe } & 0.072047000 & 0.073272000 & -0.140033000 \\ \text { O } & -2.028246000 & 1.370615000 & 2.357855000 \\ \text { C } & 2.235080000 & -1.743760000 & -0.441621000 \\ \text { H } & 2.638869000 & -2.758786000 & -0.310045000 \\ \text { O } & 1.514539000 & 1.061793000 & -0.734703000 \\ \text { C } & 3.100592000 & -0.757238000 & -1.027864000 \\ \text { N } & -1.039371000 & -0.754593000 & 1.405178000 \\ \text { C } & 4.382430000 & -1.145933000 & -1.498842000 \\ \text { H } & 4.666340000 & -2.204119000 & -1.425728000 \\ \text { N } & 1.012226000 & -1.523101000 & -0.026343000 \\ \text { C } & 5.264815000 & -0.218411000 & -2.055669000 \\ \text { H } & 6.246226000 & -0.538170000 & -2.425621000 \\ \text { C } & 4.878370000 & 1.141368000 & -2.130640000 \\ \text { H } & 5.566135000 & 1.882628000 & -2.558481000\end{array}$

Page 592 
$\begin{array}{llll}C & 3.625402000 & 1.556017000 & -1.671842000\end{array}$

$\begin{array}{llll}\text { H } & 3.316532000 & 2.604655000 & -1.734893000\end{array}$

C $\quad 2.697184000 \quad 0.621290000 \quad-1.136002000$

$\begin{array}{llll}\text { C } & 0.262906000 & -2.641718000 & 0.573157000\end{array}$

H $\quad 0.931725000 \quad-3.474399000 \quad 0.851826000$

$\begin{array}{llll}\text { H } & -0.457828000 & -3.019079000 & -0.176940000\end{array}$

$\begin{array}{llll}\text { C } & -0.447566000 & -2.069506000 & 1.785148000\end{array}$

$\begin{array}{llll}\text { H } & -1.247274000 & -2.732890000 & 2.154868000\end{array}$

H $\quad 0.258191000 \quad-1.876686000 \quad 2.605447000$

$\begin{array}{llll}\text { C } & -2.085301000 & -0.787513000 & 0.519186000\end{array}$

$\begin{array}{llll}\text { C } & -3.236064000 & 0.050358000 & 0.726719000\end{array}$

$\begin{array}{llll}\text { C } & -4.464302000 & -0.207233000 & 0.066384000\end{array}$

H $\quad-4.513325000-1.034779000 \quad-0.651347000$

$\begin{array}{llll}\text { C } & -5.580275000 & 0.601436000 & 0.289910000\end{array}$

$\begin{array}{llll}\text { H } & -6.517197000 & 0.407251000 & -0.244641000\end{array}$

C $\quad-5.480452000 \quad 1.687353000 \quad 1.191509000$

$\begin{array}{llll}\text { H } & -6.346990000 & 2.339369000 & 1.360455000\end{array}$

$\begin{array}{llll}\text { C } & -4.287952000 & 1.936479000 & 1.887498000\end{array}$

$\begin{array}{llll}\text { H } & -4.204081000 & 2.766931000 & 2.598931000\end{array}$

$\begin{array}{llll}\text { C } & -3.158891000 & 1.115067000 & 1.676116000\end{array}$

$\begin{array}{llll}\text { H } & -2.196110000 & -1.687522000 & -0.097503000\end{array}$

$\begin{array}{llll}\text { B } & -0.823370000 & 0.501193000 & 2.377570000\end{array}$

$\begin{array}{llll}\text { O } & 0.334374000 & 1.195269000 & 1.774603000\end{array}$

$\begin{array}{llll}0 & -0.438628000 & 0.140171000 & 3.717672000\end{array}$

$\begin{array}{llll}\text { C } & 1.440371000 & 1.213979000 & 2.742007000\end{array}$

$\begin{array}{llll}\text { C } & 0.654122000 & 1.032707000 & 4.097250000\end{array}$

C $\quad 0.056255000 \quad 2.360065000 \quad 4.598164000$

$\begin{array}{llll}\text { C } & 1.458679000 & 0.358616000 & 5.209321000\end{array}$

$\begin{array}{llll}\text { C } & 2.186356000 & 2.536420000 & 2.584579000\end{array}$

$\begin{array}{llll}\text { C } & 2.411861000 & 0.062620000 & 2.460713000\end{array}$

$\begin{array}{llll}\text { H } & 2.633057000 & 2.578269000 & 1.576293000\end{array}$

$\begin{array}{llll}\text { H } & 1.512572000 & 3.397364000 & 2.678229000\end{array}$

$\begin{array}{llll}H & 2.781870000 & 0.150876000 & 1.428863000\end{array}$

$\begin{array}{llll}H & 0.841166000 & 3.029356000 & 4.992481000\end{array}$

$\begin{array}{llll}H & -0.480866000 & 2.883644000 & 3.789585000\end{array}$

$\begin{array}{llll}\text { H } & 1.936275000 & -0.922900000 & 2.572071000\end{array}$

$\begin{array}{llll}H & 3.278528000 & 0.111730000 & 3.142051000\end{array}$

$\begin{array}{llll}H & 2.990330000 & 2.619058000 & 3.338283000\end{array}$

$\begin{array}{llll}H & -0.659125000 & 2.146727000 & 5.411842000\end{array}$

$\begin{array}{llll}H & 2.370383000 & 0.940048000 & 5.436210000\end{array}$

$\begin{array}{llll}\text { H } & 1.753693000 & -0.665362000 & 4.926899000\end{array}$

$\begin{array}{llll}H & 0.850882000 & 0.301795000 & 6.130003000\end{array}$

$\begin{array}{llll}H & -0.115301000 & 2.560274000 & -1.678759000\end{array}$

C $\quad-2.926812000 \quad 2.045452000 \quad-1.504197000$

C $\quad-2.003649000 \quad 2.814210000 \quad-0.706008000$

$\begin{array}{llll}\text { C } & -2.747550000 & 1.007967000 & -2.394279000\end{array}$

$\begin{array}{llll}\text { H } & -1.468301000 & 0.067406000 & -3.817642000\end{array}$

C $\quad-0.658411000 \quad 3.034017000 \quad-0.851734000$

$\begin{array}{llll}\text { C } & -1.497041000 & 0.313640000 & -2.738984000\end{array}$

H $\quad-2.506103000 \quad 3.363523000 \quad 0.102803000$

C $\quad-0.452731000 \quad-0.226016000 \quad-2.030101000$

$\begin{array}{llll}\text { H } & -3.973617000 & 2.301891000 & -1.292723000\end{array}$

$\begin{array}{llll}\text { C } & -3.957328000 & 0.462019000 & -3.076881000\end{array}$

$\begin{array}{llll}\text { C } & -5.081704000 & 1.268911000 & -3.386715000\end{array}$

C $\quad-4.029639000 \quad-0.919042000 \quad-3.395443000$

H $\quad-5.043853000 \quad 2.347147000 \quad-3.186888000$

H $\quad-3.175831000 \quad-1.565311000-3.154295000$

C $\quad-6.235779000 \quad 0.715903000 \quad-3.962933000$

C $\quad-5.184231000 \quad-1.474909000 \quad-3.970067000$

$\begin{array}{llll}\text { H } & -7.090236000 & 1.364809000 & -4.195273000\end{array}$

H $\quad-5.217875000 \quad-2.549822000 \quad-4.190399000$

C $\quad-6.296362000 \quad-0.660673000 \quad-4.254944000$

H $\quad-7.198902000 \quad-1.092180000 \quad-4.706185000$

$\begin{array}{llll}\text { C } & 0.545704000 & -0.930146000 & -2.887916000\end{array}$

C $\quad 1.438301000 \quad-0.186839000 \quad-3.697388000$

$\begin{array}{llll}\text { C } & 0.633550000 & -2.340958000 & -2.928144000\end{array}$

Page S93 


$\begin{array}{lrrr}\text { H } & 1.380624000 & 0.907962000 & -3.672091000 \\ \text { H } & -0.064753000 & -2.936306000 & -2.327044000 \\ \text { C } & 2.410752000 & -0.825422000 & -4.484616000 \\ \text { C } & 1.602451000 & -2.984485000 & -3.716031000 \\ \text { H } & 3.107848000 & -0.223155000 & -5.080957000 \\ \text { H } & 1.655352000 & -4.081099000 & -3.723962000 \\ \text { C } & 2.505069000 & -2.228554000 & -4.488312000 \\ \text { H } & 3.272973000 & -2.730991000 & -5.090275000 \\ \text { C } & 0.098505000 & 3.970475000 & -0.019536000 \\ \text { C } & -0.404369000 & 4.496652000 & 1.201497000 \\ \text { C } & 1.366584000 & 4.425030000 & -0.461363000 \\ \text { H } & -1.346574000 & 4.112347000 & 1.610201000 \\ \text { H } & 1.773073000 & 4.018938000 & -1.395538000 \\ \text { C } & 0.307248000 & 5.471399000 & 1.913332000 \\ \text { C } & 2.088728000 & 5.388834000 & 0.259780000 \\ \text { H } & -0.100825000 & 5.859593000 & 2.855465000 \\ \text { H } & 3.067478000 & 5.723484000 & -0.107765000 \\ \text { C } & 1.557406000 & 5.928089000 & 1.446265000 \\ \text { H } & 2.116796000 & 6.684163000 & 2.011514000\end{array}$

\section{${ }^{1} 5 \mathrm{VI-AAAB}$}

\section{FSPE: -3494.249561733670 $E_{\mathrm{h}}$}

$\begin{array}{llll}\text { Fe } & -0.029723000 & 0.046421000 & 0.163092000\end{array}$

$\begin{array}{llll}\mathrm{O} & -1.907731000 & 1.543934000 & 2.757316000\end{array}$

C $\quad 1.958044000 \quad-1.907652000 \quad-0.331469000$

H $\quad 2.313586000 \quad-2.943629000 \quad-0.221578000$

$\begin{array}{llll}\text { O } & 1.396820000 & 0.943383000 & -0.598988000\end{array}$

$\begin{array}{llll}\text { C } \quad 2.792254000 & -0.991870000 & -1.069996000\end{array}$

N $\quad-1.084520000 \quad-0.661395000 \quad 1.818775000$

C $\quad 3.989087000 \quad-1.468673000 \quad-1.673068000$

H $\quad 4.222481000 \quad-2.539279000 \quad-1.596374000$

$\begin{array}{llll}\mathrm{N} & 0.828461000 & -1.591416000 & 0.254142000\end{array}$

$\begin{array}{llll}\text { C } & 4.858839000 & -0.608742000 & -2.347554000\end{array}$

$\begin{array}{llll}\text { H } & 5.773563000 & -0.997167000 & -2.810837000\end{array}$

$\begin{array}{llll}\text { C } & 4.550105000 & 0.771543000 & -2.416748000\end{array}$

$\begin{array}{llll}\text { H } & 5.229882000 & 1.458967000 & -2.936940000\end{array}$

$\begin{array}{llll}\text { C } \quad 3.385467000 & 1.273798000 & -1.828689000\end{array}$

$\begin{array}{llll}\text { H } & 3.142110000 & 2.341255000 & -1.868332000\end{array}$

C $\quad 2.474069000 \quad 0.412051000 \quad-1.157368000$

$\begin{array}{llll}\text { C } & 0.075289000 & -2.642227000 & 0.966540000\end{array}$

$\begin{array}{llll}\mathrm{H} & 0.724459000 & -3.494250000 & 1.233414000\end{array}$

$\begin{array}{llll}\text { H } & -0.717215000 & -3.011051000 & 0.288050000\end{array}$

$\begin{array}{llll}\text { C } & -0.508942000 & -1.983397000 & 2.204566000\end{array}$

$\begin{array}{llll}\text { H } & -1.297595000 & -2.599270000 & 2.669054000\end{array}$

H $\quad 0.272155000 \quad-1.781901000 \quad 2.951852000$

$\begin{array}{llll}\text { C } & -2.200870000 & -0.681026000 & 1.021374000\end{array}$

C $\quad-3.281639000 \quad 0.238422000 \quad 1.251929000$

$\begin{array}{llll}\text { C } & -4.562140000 & 0.033007000 & 0.675295000\end{array}$

$\begin{array}{llll}\text { H } & -4.705853000 & -0.821288000 & 0.002563000\end{array}$

$\begin{array}{llll}\text { C } & -5.609780000 & 0.921581000 & 0.923156000\end{array}$

$\begin{array}{llll}\text { H } & -6.589838000 & 0.760442000 & 0.459377000\end{array}$

$\begin{array}{llll}\text { C } & -5.388478000 & 2.042080000 & 1.759546000\end{array}$

$\begin{array}{llll}\text { H } & -6.201739000 & 2.755187000 & 1.945248000\end{array}$

$\begin{array}{llll}\text { C } & -4.143190000 & 2.245986000 & 2.370384000\end{array}$

$\begin{array}{llll}\text { H } & -3.964935000 & 3.100124000 & 3.034793000\end{array}$

C $\quad-3.083480000 \quad 1.340537000 \quad 2.138682000$

$\begin{array}{llll}\text { H } & -2.399039000 & -1.596842000 & 0.451501000\end{array}$

$\begin{array}{llll}\text { B } & -0.736219000 & 0.632335000 & 2.704804000\end{array}$

$\begin{array}{llll}\text { O } & 0.373403000 & 1.260859000 & 1.953332000\end{array}$

\begin{tabular}{llll}
\hline & -0.227905000 & 0.331010000 & 4.015559000
\end{tabular}

C $\quad 1.570369000 \quad 1.302252000 \quad 2.805694000$

$\begin{array}{llll}\text { C } & 0.915173000 & 1.216158000 & 4.237955000\end{array}$

C $\quad 0.397127000 \quad 2.584606000 \quad 4.716324000$

$\begin{array}{llll}\text { C } & 1.808336000 & 0.583112000 & 5.305398000\end{array}$ 
$\begin{array}{llll}\text { C } & 2.319352000 & 2.595832000 & 2.495183000\end{array}$

$\begin{array}{llll}\text { C } & 2.480453000 & 0.107383000 & 2.499900000\end{array}$

$\begin{array}{llll}\text { H } & 2.664153000 & 2.564903000 & 1.447152000\end{array}$

$\begin{array}{llll}\text { H } & 1.669157000 & 3.474656000 & 2.597498000\end{array}$

$\begin{array}{llll}H & 2.749299000 & 0.122769000 & 1.433497000\end{array}$

$\begin{array}{llll}H & 1.231392000 & 3.251203000 & 4.997470000\end{array}$

H $\quad-0.201368000 \quad 3.079438000 \quad 3.933038000$

$\begin{array}{llll}H & 1.989421000 & -0.853325000 & 2.716332000\end{array}$

$\begin{array}{llll}H & 3.410157000 & 0.166667000 & 3.091279000\end{array}$

$\begin{array}{llll}H & 3.194306000 & 2.708174000 & 3.160682000\end{array}$

$\begin{array}{llll}H & -0.242838000 & 2.436201000 & 5.603865000\end{array}$

$\begin{array}{llll}H & 2.749318000 & 1.152283000 & 5.411494000\end{array}$

$\begin{array}{llll}H & 2.053582000 & -0.462193000 & 5.055569000\end{array}$

$\begin{array}{llll}H & 1.290250000 & 0.592838000 & 6.280893000\end{array}$

$\begin{array}{llll}\text { H } & -0.254336000 & 2.320101000 & -1.736769000\end{array}$

C $\quad-3.036927000 \quad 1.685485000 \quad-1.426429000$

$\begin{array}{llll}\text { C } & -2.124374000 & 2.570179000 & -0.733337000\end{array}$

$\begin{array}{llll}\text { C } & -2.848925000 & 0.540595000 & -2.164832000\end{array}$

H $\quad 0.292889000 \quad-0.967277000 \quad-2.113311000$

C $\quad-0.791053000 \quad 2.822376000 \quad-0.923180000$

$\begin{array}{llll}\text { C } & -1.554926000 & -0.121622000 & -2.487980000\end{array}$

$\begin{array}{llll}\text { H } & -2.630229000 & 3.160537000 & 0.044480000\end{array}$

C $\quad-0.547970000 \quad-0.443786000 \quad-1.618076000$

H $\quad-4.085218000 \quad 1.928960000 \quad-1.211506000$

C $\quad-4.063409000 \quad-0.194161000 \quad-2.621107000$

$\begin{array}{llll}\text { C } & -5.254472000 & 0.469799000 & -3.004385000\end{array}$

$\begin{array}{llll}\text { C } & -4.067678000 & -1.612485000 & -2.610588000\end{array}$

$\begin{array}{llll}\text { H } & -5.267173000 & 1.565867000 & -3.056274000\end{array}$

H $\quad-3.153199000 \quad-2.143320000-2.316955000$

$\begin{array}{llll}\text { C } & -6.410434000 & -0.253237000 & -3.340002000\end{array}$

$\begin{array}{llll}\text { C } & -5.224193000 & -2.336931000 & -2.938721000\end{array}$

$\begin{array}{llll}\text { H } & -7.319293000 & 0.284734000 & -3.639456000\end{array}$

H $\quad-5.205706000 \quad-3.434038000 \quad-2.906844000$

C $\quad-6.404133000-1.660888000 \quad-3.304338000$

H $\quad-7.307923000 \quad-2.225646000-3.566234000$

C $\quad-1.404679000 \quad-0.498504000 \quad-3.932794000$

$\begin{array}{llll}\text { C } & -1.860981000 & 0.384853000 & -4.943265000\end{array}$

C $\quad-0.814911000 \quad-1.720117000 \quad-4.341276000$

H $\quad-2.332602000 \quad 1.331855000 \quad-4.648506000$

H $\quad-0.492427000 \quad-2.440345000-3.578199000$

C $\quad-1.713272000 \quad 0.072924000 \quad-6.304312000$

$\begin{array}{llll}\text { C } & -0.664921000 & -2.034416000 & -5.701983000\end{array}$

H $\quad-2.068471000 \quad 0.779850000 \quad-7.065817000$

H $\quad-0.211202000 \quad-2.991881000 \quad-5.990356000$

$\begin{array}{llll}\text { C } & -1.111191000 & -1.139053000 & -6.693092000\end{array}$

$\begin{array}{llll}\text { H } & -0.999337000 & -1.387515000 & -7.756247000\end{array}$

$\begin{array}{llll}\text { C } & -0.037982000 & 3.816835000 & -0.153644000\end{array}$

$\begin{array}{llll}\text { C } & -0.500191000 & 4.339614000 & 1.084092000\end{array}$

$\begin{array}{llll}\text { C } & 1.185128000 & 4.314996000 & -0.668067000\end{array}$

H $\quad-1.394053000 \quad 3.912034000 \quad 1.553693000$

$\begin{array}{llll}\text { H } & 1.564431000 & 3.907605000 & -1.613570000\end{array}$

$\begin{array}{llll}\text { C } & 0.199836000 & 5.361190000 & 1.739394000\end{array}$

$\begin{array}{llll}\text { C } & 1.895886000 & 5.327615000 & -0.004007000\end{array}$

$\begin{array}{llll}\text { H } & -0.175174000 & 5.747686000 & 2.695833000\end{array}$

$\begin{array}{llll}\text { H } & 2.837738000 & 5.699656000 & -0.427563000\end{array}$

$\begin{array}{llll}\text { C } & 1.399210000 & 5.867707000 & 1.196867000\end{array}$

$\begin{array}{llll}\text { H } & 1.949859000 & 6.661349000 & 1.717491000\end{array}$

\section{${ }^{1}$ 5VI-AABA $\quad$ FSPE: $-\mathbf{3 4 9 4 . 2 4 8 3 8 7 7 8 1 6 2 7} E_{\mathrm{h}}$}
Fe $\quad 0.082563000 \quad-0.011756000 \quad-0.079637000$
$\begin{array}{llll}\mathrm{O} & -1.909198000 & 1.025479000 & 2.693417000\end{array}$
C $\quad 2.524363000 \quad-1.402033000 \quad-0.511889000$
H $\quad 3.141802000 \quad-2.298538000-0.350827000$ 
$\begin{array}{llll}\text { O } & 1.199254000 & 1.147338000 & -0.965019000\end{array}$

$\begin{array}{llll}\text { C } & 3.093945000 & -0.336601000 & -1.290748000\end{array}$

$\begin{array}{llll}\mathrm{N} & -0.667486000 & -0.894682000 & 1.624942000\end{array}$

$\begin{array}{llll}\text { C } & 4.376265000 & -0.508926000 & -1.879549000\end{array}$

H $4.906755000 \quad-1.455238000 \quad-1.709134000$

$\begin{array}{llll}\text { N } & 1.330603000 & -1.387555000 & 0.029415000\end{array}$

$\begin{array}{llll}\text { C } & 4.950032000 & 0.484137000 & -2.675175000\end{array}$

$\begin{array}{llll}\text { H } & 5.936856000 & 0.330826000 & -3.127813000\end{array}$

$\begin{array}{llll}\text { C } & 4.239591000 & 1.689532000 & -2.897401000\end{array}$

H $4.675866000 \quad 2.472733000 \quad-3.530928000$

$\begin{array}{llll}\text { C } & 2.980766000 & 1.892975000 & -2.325178000\end{array}$

$\begin{array}{llll}\text { H } & 2.420379000 & 2.818723000 & -2.500280000\end{array}$

$\begin{array}{llll}\text { C } & 2.381180000 & 0.896239000 & -1.507359000\end{array}$

$\begin{array}{llll}\text { C } & 0.887342000 & -2.584735000 & 0.772835000\end{array}$

$\begin{array}{llll}\text { H } & 1.745775000 & -3.217459000 & 1.057917000\end{array}$

$\begin{array}{llll}H & 0.242938000 & -3.174656000 & 0.096673000\end{array}$

$\begin{array}{llll}\text { C } & 0.128508000 & -2.098137000 & 1.994903000\end{array}$

$\begin{array}{llll}H & -0.548685000 & -2.869801000 & 2.399416000\end{array}$

$\begin{array}{llll}\text { H } & 0.812583000 & -1.784698000 & 2.795761000\end{array}$

$\begin{array}{llll}\text { C } & -1.725877000 & -1.072347000 & 0.766780000\end{array}$

$\begin{array}{llll}\text { C } & -2.946991000 & -0.322736000 & 0.961207000\end{array}$

$\begin{array}{llll}\text { C } & -4.125777000 & -0.647145000 & 0.244462000\end{array}$

$\begin{array}{llll}\text { H } & -4.069516000 & -1.420324000 & -0.532068000\end{array}$

$\begin{array}{llll}\text { C } & -5.329168000 & 0.007894000 & 0.513919000\end{array}$

$\begin{array}{llll}\text { H } & -6.233949000 & -0.242625000 & -0.049873000\end{array}$

$\begin{array}{llll}\text { C } & -5.364677000 & 1.017911000 & 1.501655000\end{array}$

$\begin{array}{llll}\text { H } & -6.301144000 & 1.553941000 & 1.699548000\end{array}$

$\begin{array}{llll}\text { C } & -4.214661000 & 1.350058000 & 2.228843000\end{array}$

$\begin{array}{llll}\text { H } & -4.230245000 & 2.130772000 & 2.999020000\end{array}$

$\begin{array}{llll}\text { C } & -2.993504000 & 0.685798000 & 1.970680000\end{array}$

$\begin{array}{llll}\text { H } & -1.797703000 & -2.040407000 & 0.256261000\end{array}$

$\begin{array}{llll}\text { B } & -0.566912000 & 0.400234000 & 2.576438000\end{array}$

$\begin{array}{llll}\text { O } & 0.398931000 & 1.283812000 & 1.895595000\end{array}$

$\begin{array}{llll}0 & -0.020363000 & 0.094556000 & 3.874534000\end{array}$

$\begin{array}{llll}\text { C } & 1.581558000 & 1.432385000 & 2.750489000\end{array}$

$\begin{array}{llll}\text { C } & 0.977615000 & 1.115459000 & 4.170812000\end{array}$

$\begin{array}{llll}\text { C } & 0.259062000 & 2.335375000 & 4.775986000\end{array}$

$\begin{array}{llll}\text { C } & 1.980071000 & 0.535882000 & 5.169686000\end{array}$

$\begin{array}{llll}\text { C } & 2.130605000 & 2.846913000 & 2.588838000\end{array}$

$\begin{array}{llll}\text { C } & 2.667295000 & 0.438809000 & 2.320808000\end{array}$

$\begin{array}{llll}\text { H } & 2.498124000 & 2.981688000 & 1.557782000\end{array}$

$\begin{array}{llll}H & 1.357840000 & 3.604986000 & 2.764868000\end{array}$

$\begin{array}{llll}H & 2.885199000 & 0.597639000 & 1.253077000\end{array}$

$\begin{array}{llll}H & 0.977973000 & 3.102866000 & 5.112875000\end{array}$

$\begin{array}{llll}H & -0.425732000 & 2.792414000 & 4.041492000\end{array}$

$\begin{array}{llll}H & 2.356270000 & -0.607450000 & 2.454375000\end{array}$

$\begin{array}{llll}\text { H } & 3.597018000 & 0.601483000 & 2.892928000\end{array}$

$\begin{array}{llll}H & 2.969014000 & 3.014780000 & 3.289161000\end{array}$

$\begin{array}{llll}H & -0.331330000 & 2.006398000 & 5.649396000\end{array}$

$\begin{array}{llll}\text { H } & 2.820807000 & 1.235346000 & 5.326721000\end{array}$

$\begin{array}{llll}H & 2.384067000 & -0.428040000 & 4.818446000\end{array}$

$\begin{array}{llll}\text { H } & 1.486271000 & 0.368616000 & 6.143629000\end{array}$

$\begin{array}{llll}\text { H } & -0.463149000 & 2.771085000 & -1.626668000\end{array}$

$\begin{array}{llll}\text { C } & -3.090100000 & 1.867216000 & -1.530583000\end{array}$

$\begin{array}{llll}\text { C } & -2.231221000 & 2.456223000 & -0.498207000\end{array}$

$\begin{array}{llll}\text { C } & -2.753259000 & 0.869956000 & -2.420301000\end{array}$

$\begin{array}{llll}\text { H } & -1.764601000 & -0.601431000 & -3.536261000\end{array}$

$\begin{array}{llll}\text { C } & -0.978084000 & 2.962015000 & -0.676891000\end{array}$

$\begin{array}{llll}\text { C } & -1.671195000 & -0.084434000 & -2.563586000\end{array}$

$\begin{array}{llll}\text { H } & -2.751132000 & 2.696835000 & 0.437900000\end{array}$

$\begin{array}{llll}\text { C } & -0.657462000 & -0.621940000 & -1.795906000\end{array}$

$\begin{array}{llll}\text { H } & -3.501628000 & 0.716465000 & -3.211985000\end{array}$

$\begin{array}{llll}\text { C } & -4.448341000 & 2.479707000 & -1.609330000\end{array}$

$\begin{array}{llll}\text { C } & -4.674133000 & 3.801508000 & -1.142729000\end{array}$

$\begin{array}{llll}\text { C } & -5.566046000 & 1.771614000 & -2.124622000\end{array}$

$\begin{array}{llll}\text { H } & -3.832093000 & 4.388612000 & -0.754566000\end{array}$

Page 596 


$\begin{array}{lrrr}\text { H } & -5.443113000 & 0.731038000 & -2.446742000 \\ \text { C } & -5.951180000 & 4.385181000 & -1.185481000 \\ \text { C } & -6.839330000 & 2.356283000 & -2.179168000 \\ \text { H } & -6.090883000 & 5.410278000 & -0.817905000 \\ \text { H } & -7.684169000 & 1.776287000 & -2.573185000 \\ \text { C } & -7.043840000 & 3.666489000 & -1.703787000 \\ \text { H } & -8.043200000 & 4.119036000 & -1.734453000 \\ \text { C } & 0.066828000 & -1.741683000 & -2.466226000 \\ \text { C } & 1.125218000 & -1.483347000 & -3.370109000 \\ \text { C } & -0.313270000 & -3.088628000 & -2.252944000 \\ \text { H } & 1.417067000 & -0.444264000 & -3.564015000 \\ \text { H } & -1.172199000 & -3.306369000 & -1.604480000 \\ \text { C } & 1.816401000 & -2.536381000 & -3.992557000 \\ \text { C } & 0.377318000 & -4.143957000 & -2.873990000 \\ \text { H } & 2.645010000 & -2.309766000 & -4.675981000 \\ \text { H } & 0.066050000 & -5.180378000 & -2.688992000 \\ \text { C } & 1.455721000 & -3.872316000 & -3.737089000 \\ \text { H } & 2.000202000 & -4.695097000 & -4.217839000 \\ \text { C } & -0.329472000 & 3.913057000 & 0.232775000 \\ \text { C } & -0.900692000 & 4.306818000 & 1.472434000 \\ \text { C } & 0.845706000 & 4.578140000 & -0.195570000 \\ \text { H } & -1.783320000 & 3.782346000 & 1.857006000 \\ \text { H } & 1.312749000 & 4.270433000 & -1.139348000 \\ \text { C } & -0.350913000 & 5.358899000 & 2.215890000 \\ \text { C } & 1.404671000 & 5.626542000 & 0.554219000 \\ \text { H } & -0.813123000 & 5.648704000 & 3.168180000 \\ \text { H } & 2.311495000 & 6.129887000 & 0.194986000 \\ \text { C } & 0.800486000 & 6.033733000 & 1.757215000 \\ \text { H } & 1.227634000 & 6.858261000 & 2.341755000\end{array}$

\section{${ }^{1}$ 5VI-AABB $\quad$ FSPE: $-3494.249672829259 E_{\mathrm{h}}$}

$\begin{array}{lrrr}\text { Fe } & -0.106143000 & 0.006322000 & 0.181069000 \\ \text { O } & -1.838052000 & 1.176907000 & 3.143892000 \\ \text { C } & 2.067437000 & -1.681292000 & -0.475652000 \\ \text { H } & 2.559350000 & -2.663613000 & -0.404749000 \\ \text { O } & 1.093173000 & 1.056216000 & -0.734670000 \\ \text { C } & 2.687351000 & -0.684938000 & -1.311868000 \\ \text { N } & -0.910084000 & -0.828800000 & 1.929837000 \\ \text { C } & 3.868270000 & -1.015953000 & -2.034119000 \\ \text { H } & 4.261068000 & -2.038558000 & -1.954215000 \\ \text { N } & 0.978386000 & -1.498996000 & 0.231943000 \\ \text { C } & 4.523517000 & -0.073858000 & -2.829166000 \\ \text { H } & 5.428803000 & -0.349679000 & -3.382811000 \\ \text { C } & 4.008287000 & 1.243580000 & -2.908773000 \\ \text { H } & 4.516049000 & 1.992457000 & -3.530468000 \\ \text { C } & 2.854859000 & 1.603804000 & -2.206860000 \\ \text { H } & 2.448726000 & 2.620445000 & -2.260868000 \\ \text { C } & 2.163024000 & 0.655186000 & -1.403649000 \\ \text { C } & 0.390617000 & -2.654118000 & 0.939521000 \\ \text { H } & 1.150420000 & -3.426539000 & 1.151983000 \\ \text { H } & -0.372789000 & -3.097967000 & 0.271175000 \\ \text { C } & -0.228519000 & -2.120355000 & 2.219179000 \\ \text { H } & -0.954144000 & -2.826999000 & 2.657107000 \\ \text { H } & 0.544721000 & -1.900031000 & 2.969871000 \\ \text { C } & -2.029605000 & -0.840220000 & 1.137153000 \\ \text { C } & -3.106612000 & 0.091031000 & 1.385158000 \\ \text { C } & -4.329591000 & 0.009165000 & 0.673295000 \\ \text { H } & -4.421681000 & -0.734537000 & -0.127740000 \\ \text { C } & -5.384449000 & 0.878922000 & 0.959389000 \\ \text { H } & -6.313377000 & 0.825643000 & 0.381858000 \\ \text { C } & -5.231311000 & 1.851981000 & 1.972845000 \\ \text { H } & -6.051651000 & 2.546972000 & 2.191119000 \\ \text { C } & -4.038585000 & 1.942699000 & 2.702730000\end{array}$


$\begin{array}{llll}\text { H } & -3.906770000 & 2.688967000 & 3.495395000\end{array}$

$\begin{array}{llll}\text { C } & -2.964494000 & 1.068920000 & 2.417066000\end{array}$

$\begin{array}{llll}\text { H } & -2.254496000 & -1.763975000 & 0.589809000\end{array}$

$\begin{array}{llll}\text { B } & -0.582395000 & 0.438052000 & 2.868630000\end{array}$

$\begin{array}{llll}\text { O } & 0.368011000 & 1.237043000 & 2.066516000\end{array}$

$\begin{array}{llll}\text { O } & 0.084444000 & 0.092934000 & 4.094585000\end{array}$

C $\quad 1.634663000 \quad 1.337233000 \quad 2.804250000$

$\begin{array}{llll}\text { C } & 1.148907000 & 1.076396000 & 4.280155000\end{array}$

$\begin{array}{llll}\text { C } & 0.546463000 & 2.340660000 & 4.919667000\end{array}$

$\begin{array}{llll}\text { C } & 2.209258000 & 0.468254000 & 5.198614000\end{array}$

$\begin{array}{llll}\text { C } & 2.225017000 & 2.720472000 & 2.547019000\end{array}$

$\begin{array}{llll}\text { C } & 2.616969000 & 0.270440000 & 2.309860000\end{array}$

$\begin{array}{llll}\text { H } & 2.493172000 & 2.806889000 & 1.480297000\end{array}$

$\begin{array}{llll}\text { H } & 1.500707000 & 3.515355000 & 2.768179000\end{array}$

$\begin{array}{llll}H & 2.759464000 & 0.392368000 & 1.225353000\end{array}$

$\begin{array}{llll}\text { H } & 1.332359000 & 3.073062000 & 5.175550000\end{array}$

$\begin{array}{llll}H & -0.177575000 & 2.821912000 & 4.240924000\end{array}$

$\begin{array}{llll}H & 2.247317000 & -0.749282000 & 2.496077000\end{array}$

$\begin{array}{llll}\text { H } & 3.597536000 & 0.385339000 & 2.803124000\end{array}$

$\begin{array}{llll}H & 3.133071000 & 2.873710000 & 3.158083000\end{array}$

$\begin{array}{llll}H & 0.021060000 & 2.057554000 & 5.848738000\end{array}$

$\begin{array}{llll}\text { H } & 3.094542000 & 1.126621000 & 5.257725000\end{array}$

$\begin{array}{llll}H & 2.529236000 & -0.523888000 & 4.840061000\end{array}$

$\begin{array}{llll}\text { H } & 1.800011000 & 0.351753000 & 6.218028000\end{array}$

H $\quad-0.500825000 \quad 2.649199000 \quad-1.643072000$

C $\quad-3.109294000 \quad 1.696956000 \quad-1.647842000$

$\begin{array}{llll}\text { C } & -2.313506000 & 2.340104000 & -0.592696000\end{array}$

$\begin{array}{llll}\text { C } & -2.748078000 & 0.639747000 & -2.453034000\end{array}$

H $\quad-0.124099000 \quad-1.541216000 \quad-1.798712000$

$\begin{array}{llll}\text { C } & -1.055661000 & 2.851643000 & -0.718465000\end{array}$

$\begin{array}{llll}\text { C } & -1.667087000 & -0.342492000 & -2.451236000\end{array}$

$\begin{array}{llll}\text { H } & -2.889258000 & 2.604016000 & 0.305661000\end{array}$

$\begin{array}{llll}\text { C } & -0.757884000 & -0.694413000 & -1.473803000\end{array}$

$\begin{array}{llll}\text { H } & -3.471092000 & 0.439578000 & -3.257942000\end{array}$

C $\quad-4.458879000 \quad 2.307664000 \quad-1.835031000$

C $\quad-4.683434000 \quad 3.671224000 \quad-1.514323000$

$\begin{array}{llll}\text { C } & -5.568335000 & 1.552797000 & -2.298063000\end{array}$

H $\quad-3.846054000 \quad 4.286502000 \quad-1.160954000$

H $\quad-5.445885000 \quad 0.481130000 \quad-2.497901000$

$\begin{array}{llll}\text { C } & -5.953408000 & 4.254914000 & -1.653872000\end{array}$

$\begin{array}{llll}\text { C } & -6.835378000 & 2.136101000 & -2.447499000\end{array}$

$\begin{array}{llll}\text { H } & -6.094845000 & 5.313361000 & -1.398843000\end{array}$

H $\quad-7.676172000 \quad 1.522423000 \quad-2.796418000$

$\begin{array}{llll}\text { C } & -7.038435000 & 3.491475000 & -2.122295000\end{array}$

$\begin{array}{llll}\text { H } & -8.032584000 & 3.944413000 & -2.226723000\end{array}$

$\begin{array}{llll}\text { C } & -1.601455000 & -1.146037000 & -3.724859000\end{array}$

C $\quad-1.644140000 \quad-0.498297000 \quad-4.984878000$

C $\quad-1.492515000 \quad-2.558916000 \quad-3.713900000$

H $\quad-1.724091000 \quad 0.596506000 \quad-5.022653000$

H $\quad-1.491913000 \quad-3.087806000-2.751679000$

C $\quad-1.561861000-1.229101000-6.183284000$

$\begin{array}{llll}\text { C } & -1.409167000 & -3.292201000 & -4.909903000\end{array}$

H $\quad-1.585046000 \quad-0.699269000 \quad-7.144732000$

H $\quad-1.332836000 \quad-4.386826000 \quad-4.870895000$

$\begin{array}{llll}\text { C } & -1.441240000 & -2.631207000 & -6.152857000\end{array}$

$\begin{array}{llll}\text { H } & -1.378636000 & -3.203385000 & -7.087297000\end{array}$

$\begin{array}{llll}\text { C } & -0.429693000 & 3.794043000 & 0.217363000\end{array}$

$\begin{array}{llll}\text { C } & -0.988360000 & 4.117031000 & 1.482113000\end{array}$

$\begin{array}{llll}\text { C } & 0.727499000 & 4.499313000 & -0.195711000\end{array}$

$\begin{array}{llll}\text { H } & -1.850478000 & 3.554815000 & 1.857273000\end{array}$

$\begin{array}{llll}\text { H } & 1.185959000 & 4.247546000 & -1.160478000\end{array}$

$\begin{array}{llll}\text { C } & -0.445336000 & 5.141540000 & 2.267669000\end{array}$

$\begin{array}{llll}\text { C } & 1.277730000 & 5.522608000 & 0.594037000\end{array}$

$\begin{array}{llll}\text { H } & -0.896655000 & 5.375795000 & 3.240218000\end{array}$

$\begin{array}{llll}\text { H } & 2.169753000 & 6.059325000 & 0.246282000\end{array}$

$\begin{array}{llll}\text { C } & 0.685173000 & 5.859829000 & 1.824187000\end{array}$

Page 598 


\section{${ }^{1}$ 5VI-ABAA $\quad$ FSPE: $-3494.232155967473 E_{\mathrm{h}}$}

\begin{tabular}{|c|c|c|c|}
\hline $\mathrm{Fe}$ & 0.113866000 & 0.355387000 & 0.151638000 \\
\hline $\mathrm{O}$ & -2.301815000 & 0.435729000 & 2.875710000 \\
\hline r & 2.545215000 & -1.153518000 & -0.629552000 \\
\hline $\mathrm{H}$ & 3.094920000 & -2.103210000 & -0.750961000 \\
\hline b & 1.425018000 & 1.564433000 & -0.402449000 \\
\hline C & 3.175926000 & 0.027982000 & -1.144645000 \\
\hline$N$ & -0.753585000 & -1.012929000 & 1.535065000 \\
\hline C & 4.438383000 & -0.088426000 & -1.798304000 \\
\hline$t$ & 4.853347000 & -1.093544000 & -1.954719000 \\
\hline v & 1.397643000 & -1.168949000 & -0.002474000 \\
\hline$C$ & 5.140107000 & 1.035359000 & -2.224895000 \\
\hline-1 & 6.105106000 & 0.928206000 & -2.734315000 \\
\hline C & 4.598578000 & 2.325833000 & -1.977366000 \\
\hline H & 5.147258000 & 3.218833000 & -2.304057000 \\
\hline C & 3.373328000 & 2.474711000 & -1.333039000 \\
\hline $\mathrm{H}$ & 2.929962000 & 3.462202000 & -1.163347000 \\
\hline C & 2.610884000 & 1.336069000 & -0.933749000 \\
\hline C & 0.929601000 & -2.467356000 & 0.518841000 \\
\hline $\mathrm{H}$ & 1.767089000 & -3.158214000 & 0.722598000 \\
\hline $\mathrm{H}$ & 0.287670000 & -2.943562000 & -0.245505000 \\
\hline C & 0.155227000 & -2.164233000 & 1.783272000 \\
\hline $\mathrm{H}$ & -0.443549000 & -3.026808000 & 2.126309000 \\
\hline $\mathrm{H}$ & 0.829605000 & -1.859780000 & 2.597088000 \\
\hline C & -1.759690000 & -1.175687000 & 0.614686000 \\
\hline C & -3.086955000 & -0.698160000 & 0.885644000 \\
\hline C & -4.193464000 & -1.070384000 & 0.078837000 \\
\hline $\mathrm{H}$ & -4.013258000 & -1.658688000 & -0.828595000 \\
\hline C & -5.494932000 & -0.716737000 & 0.442642000 \\
\hline $\mathrm{H}$ & -6.342807000 & -1.011300000 & -0.187025000 \\
\hline C & -5.709991000 & 0.017503000 & 1.631081000 \\
\hline $\mathrm{H}$ & -6.729213000 & 0.302894000 & 1.920095000 \\
\hline C & -4.634165000 & 0.401044000 & 2.441238000 \\
\hline $\mathrm{H}$ & -4.786212000 & 0.988329000 & 3.353630000 \\
\hline C & -3.316701000 & 0.062714000 & 2.072930000 \\
\hline $\mathrm{H}$ & -1.620658000 & -1.959314000 & -0.139580000 \\
\hline B & -0.886904000 & 0.102580000 & 2.654264000 \\
\hline 0 & -0.075999000 & 1.221550000 & 2.029676000 \\
\hline 0 & -0.242698000 & -0.178825000 & 3.907505000 \\
\hline $\mathrm{C}$ & 1.068387000 & 1.521519000 & 2.922418000 \\
\hline C & 0.472645000 & 1.032668000 & 4.297180000 \\
\hline $\mathrm{C}$ & -0.522362000 & 2.046209000 & 4.892449000 \\
\hline $\mathrm{C}$ & 1.521163000 & 0.651703000 & 5.343302000 \\
\hline $\mathrm{C}$ & 1.344684000 & 3.018331000 & 2.794293000 \\
\hline $\mathrm{C}$ & 2.322558000 & 0.726734000 & 2.543677000 \\
\hline $\mathrm{H}$ & 1.662374000 & 3.226641000 & 1.757450000 \\
\hline $\mathrm{H}$ & 0.448600000 & 3.621590000 & 3.009346000 \\
\hline $\mathrm{H}$ & 2.624888000 & 0.966921000 & 1.516510000 \\
\hline $\mathrm{H}$ & 0.004441000 & 2.930432000 & 5.292249000 \\
\hline $\mathrm{H}$ & -1.260201000 & 2.378153000 & 4.142839000 \\
\hline $\mathrm{H}$ & 2.157187000 & -0.358668000 & 2.611491000 \\
\hline $\mathrm{H}$ & 3.152611000 & 0.999428000 & 3.216886000 \\
\hline $\mathrm{H}$ & 2.150127000 & 3.325292000 & 3.485110000 \\
\hline $\mathrm{H}$ & -1.069089000 & 1.564761000 & 5.721901000 \\
\hline $\mathrm{H}$ & 2.188030000 & 1.506313000 & 5.556022000 \\
\hline $\mathrm{H}$ & 2.132663000 & -0.201817000 & 5.008942000 \\
\hline $\mathrm{H}$ & 1.017800000 & 0.367786000 & 6.284422000 \\
\hline $\mathrm{H}$ & -1.289543000 & -0.175013000 & -2.259352000 \\
\hline C & 0.838323000 & 1.382709000 & -3.303433000 \\
\hline C & 0.640372000 & -0.028137000 & -3.187402000 \\
\hline C & 0.160461000 & 2.429856000 & -2.694042000 \\
\hline
\end{tabular}




$\begin{array}{lrrr}\text { H } & -1.668573000 & 3.196079000 & -1.939285000 \\ \text { C } & -0.409548000 & -0.726271000 & -2.616448000 \\ \text { C } & -0.953916000 & 2.375301000 & -1.742191000 \\ \text { H } & 1.444143000 & -0.626263000 & -3.641501000 \\ \text { C } & -1.178232000 & 1.713986000 & -0.547764000 \\ \text { H } & 1.723246000 & 1.662271000 & -3.889966000 \\ \text { C } & 0.632021000 & 3.813637000 & -2.986984000 \\ \text { C } & 1.132135000 & 4.199192000 & -4.255887000 \\ \text { C } & 0.630638000 & 4.782915000 & -1.950309000 \\ \text { H } & 1.115782000 & 3.478685000 & -5.083766000 \\ \text { H } & 0.267949000 & 4.486759000 & -0.957659000 \\ \text { C } & 1.623799000 & 5.496146000 & -4.476263000 \\ \text { C } & 1.125871000 & 6.078364000 & -2.168107000 \\ \text { H } & 1.998402000 & 5.773439000 & -5.470227000 \\ \text { H } & 1.129114000 & 6.805361000 & -1.345451000 \\ \text { C } & 1.626015000 & 6.442323000 & -3.433287000 \\ \text { H } & 2.010078000 & 7.455740000 & -3.606645000 \\ \text { C } & -2.396214000 & 2.236612000 & 0.136192000 \\ \text { C } & -3.670911000 & 2.111999000 & -0.473395000 \\ \text { C } & -2.300116000 & 3.027656000 & 1.306218000 \\ \text { H } & -3.763260000 & 1.499078000 & -1.378177000 \\ \text { H } & -1.321666000 & 3.134407000 & 1.779120000 \\ \text { C } & -4.800964000 & 2.746899000 & 0.063624000 \\ \text { C } & -3.428787000 & 3.665984000 & 1.844484000 \\ \text { H } & -5.777985000 & 2.615494000 & -0.418862000 \\ \text { H } & -3.323307000 & 4.278506000 & 2.749936000 \\ \text { C } & -4.686271000 & 3.529131000 & 1.227667000 \\ \text { H } & -5.570123000 & 4.021184000 & 1.653816000 \\ \text { C } & -0.503959000 & -2.186730000 & -2.626013000 \\ \text { C } & 0.627267000 & -3.033696000 & -2.793923000 \\ \text { C } & -1.765736000 & -2.812584000 & -2.432236000 \\ \text { H } & 1.621855000 & -2.592251000 & -2.929539000 \\ \text { H } & -2.658797000 & -2.181034000 & -2.341698000 \\ \text { C } & 0.501503000 & -4.429026000 & -2.742060000 \\ \text { C } & -1.891724000 & -4.209132000 & -2.375242000 \\ \text { H } & 1.394792000 & -5.056242000 & -2.856952000 \\ \text { H } & -2.880228000 & -4.659139000 & -2.217589000 \\ \text { C } & -0.755747000 & -5.027854000 & -2.522314000 \\ \text { H } & -0.848014000 & -6.119697000 & -2.468891000\end{array}$

\section{${ }^{1}$ 5VI-ABAB}

$\begin{array}{lrrr}\text { Fe } & 0.179299000 & 0.229063000 & 0.218450000 \\ \text { O } & -2.259517000 & 0.558958000 & 2.881878000 \\ \text { C } & 2.488304000 & -1.375711000 & -0.634501000 \\ \text { H } & 3.014805000 & -2.340610000 & -0.734811000 \\ \text { O } & 1.447212000 & 1.388602000 & -0.468850000 \\ \text { C } & 3.091180000 & -0.237887000 & -1.270054000 \\ \text { N } & -0.755432000 & -1.051864000 & 1.668108000 \\ \text { C } & 4.288769000 & -0.422421000 & -2.023727000 \\ \text { H } & 4.678903000 & -1.442830000 & -2.139685000 \\ \text { N } & 1.381762000 & -1.329198000 & 0.064451000 \\ \text { C } & 4.956252000 & 0.653552000 & -2.601654000 \\ \text { H } & 5.869037000 & 0.491120000 & -3.187027000 \\ \text { C } & 4.446974000 & 1.967601000 & -2.417754000 \\ \text { H } & 4.966450000 & 2.821767000 & -2.871309000 \\ \text { C } & 3.287979000 & 2.185831000 & -1.677264000 \\ \text { H } & 2.863907000 & 3.188674000 & -1.548480000 \\ \text { C } & 2.565484000 & 1.095121000 & -1.107297000 \\ \text { C } & 0.895522000 & -2.584341000 & 0.670431000 \\ \text { H } & 1.723950000 & -3.276694000 & 0.905358000 \\ \text { H } & 0.238913000 & -3.092750000 & -0.062460000 \\ \text { C } & 0.143476000 & -2.206600000 & 1.937505000 \\ \text { H } & -0.454504000 & -3.049330000 & 2.328372000\end{array}$




\begin{tabular}{|c|c|c|c|}
\hline & 0.841109000 & -1.879553000 & 2.722812000 \\
\hline & -1.751227000 & -1.219277000 & 0.739475000 \\
\hline & -2.997426000 & -0.513261000 & 0.838862000 \\
\hline & -4.009082000 & -0.634090000 & -0.149646000 \\
\hline & -3.843362000 & -1.309632000 & -0.997391000 \\
\hline & -5.192363000 & 0.102503000 & -0.059713000 \\
\hline & -5.960328000 & 0.007457000 & -0.836213000 \\
\hline & -5.389970000 & 0.975722000 & 1.034178000 \\
\hline & -6.312388000 & 1.565188000 & 1.104225000 \\
\hline & -4.409940000 & 1.110988000 & 2.026598000 \\
\hline & -4.543286000 & 1.796446000 & 2.871981000 \\
\hline & -3.202232000 & 0.388680000 & 1.929573000 \\
\hline 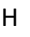 & -1.656248000 & -2.062339000 & 0.045727000 \\
\hline$B$ & -0.853120000 & 0.147872000 & 2.715261000 \\
\hline 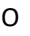 & -0.031617000 & 1.225803000 & 2.043858000 \\
\hline & -0.228264000 & -0.093478000 & 3.980344000 \\
\hline e & 1.116879000 & 1.546902000 & 2.918760000 \\
\hline C & 0.534891000 & 1.106190000 & 4.319198000 \\
\hline C & -0.417021000 & 2.160189000 & 4.911904000 \\
\hline c & 1.595240000 & 0.718127000 & 5.350609000 \\
\hline C & 1.389804000 & 3.039171000 & 2.740111000 \\
\hline C & 2.362317000 & 0.736611000 & 2.541570000 \\
\hline $\mathrm{H}$ & 1.735787000 & 3.210515000 & 1.705498000 \\
\hline H & 0.477612000 & 3.635469000 & 2.899249000 \\
\hline H & 2.647999000 & 0.953298000 & 1.502772000 \\
\hline H & 0.141335000 & 3.042998000 & 5.269786000 \\
\hline $\mathrm{H}$ & -1.165538000 & 2.487460000 & 4.171426000 \\
\hline $\mathrm{H}$ & 2.189820000 & -0.346657000 & 2.632744000 \\
\hline $\mathrm{H}$ & 3.205215000 & 1.016948000 & 3.195500000 \\
\hline H & 2.172045000 & 3.381788000 & 3.441039000 \\
\hline $\mathrm{H}$ & -0.950878000 & 1.716794000 & 5.770543000 \\
\hline $\mathrm{H}$ & 2.289569000 & 1.558316000 & 5.530364000 \\
\hline $\mathrm{H}$ & 2.176039000 & -0.158602000 & 5.021389000 \\
\hline $\mathrm{H}$ & 1.105712000 & 0.467932000 & 6.308459000 \\
\hline $\mathrm{H}$ & -1.411040000 & -0.329165000 & -2.103865000 \\
\hline C & 0.469611000 & 1.489059000 & -3.244779000 \\
\hline C & 0.386104000 & 0.054778000 & -3.207774000 \\
\hline C & -0.182313000 & 2.456066000 & -2.499337000 \\
\hline $\mathrm{H}$ & -2.035667000 & 1.516175000 & 0.288215000 \\
\hline C & -0.518571000 & -0.769929000 & -2.566336000 \\
\hline C & -1.249682000 & 2.253761000 & -1.484140000 \\
\hline $\mathrm{H}$ & 1.194912000 & -0.440815000 & -3.765253000 \\
\hline C & -1.156658000 & 1.451157000 & -0.372714000 \\
\hline $\mathrm{H}$ & 1.292171000 & 1.857982000 & -3.871964000 \\
\hline c & 0.276766000 & 3.864750000 & -2.648904000 \\
\hline c & 0.659951000 & 4.421347000 & -3.893165000 \\
\hline C & 0.396698000 & 4.670831000 & -1.488406000 \\
\hline $\mathrm{H}$ & 0.546194000 & 3.824621000 & -4.807501000 \\
\hline $\mathrm{H}$ & 0.118426000 & 4.234570000 & -0.520682000 \\
\hline C & 1.154803000 & 5.733415000 & -3.974383000 \\
\hline C & 0.898653000 & 5.979023000 & -1.567368000 \\
\hline $\mathrm{H}$ & 1.435882000 & 6.149289000 & -4.950783000 \\
\hline $\mathrm{H}$ & 0.998165000 & 6.580392000 & -0.654209000 \\
\hline c & 1.279714000 & 6.518343000 & -2.811900000 \\
\hline $\mathrm{H}$ & 1.666488000 & 7.543492000 & -2.876077000 \\
\hline c & -2.485402000 & 3.074421000 & -1.692188000 \\
\hline C & -2.886494000 & 3.438066000 & -3.004595000 \\
\hline C & -3.319221000 & 3.487428000 & -0.620838000 \\
\hline $\mathrm{H}$ & -2.264731000 & 3.130816000 & -3.855614000 \\
\hline H & -3.035181000 & 3.242311000 & 0.409696000 \\
\hline c & -4.066764000 & 4.164487000 & -3.236787000 \\
\hline c & -4.502876000 & 4.204095000 & -0.849905000 \\
\hline $\mathrm{H}$ & -4.349837000 & 4.424410000 & -4.265655000 \\
\hline $\mathrm{H}$ & -5.126510000 & 4.501760000 & 0.003395000 \\
\hline c & -4.887117000 & 4.551001000 & -2.160340000 \\
\hline $\mathrm{H}$ & -5.809922000 & 5.117922000 & -2.338734000 \\
\hline
\end{tabular}


C $\quad-0.428858000 \quad-2.232269000 \quad-2.564829000$

$\begin{array}{llll}\text { C } & 0.786492000 & -2.925944000 & -2.819779000\end{array}$

$\begin{array}{llll}\text { C } & -1.572906000 & -3.014743000 & -2.248837000\end{array}$

H $\quad 1.693381000 \quad-2.359279000 \quad-3.061566000$

$\begin{array}{llll}H & -2.533688000 & -2.510433000 & -2.086360000\end{array}$

C $\quad 0.857376000 \quad-4.323230000 \quad-2.728681000$

$\begin{array}{llll}\text { C } & -1.503546000 & -4.413783000 & -2.157394000\end{array}$

H $\quad 1.814504000 \quad-4.828361000 \quad-2.911337000$

$\begin{array}{llll}H & -2.404795000 & -4.986501000 & -1.904396000\end{array}$

C $\quad-0.283321000 \quad-5.077495000-2.386903000$

H $\quad-0.221038000 \quad-6.169518000 \quad-2.302782000$

\section{${ }^{1}$ 5VI-ABBA}

\section{FSPE: -3494.231800152516 $E_{\mathrm{h}}$}

\begin{tabular}{|c|c|c|c|}
\hline & & & \\
\hline & -2.392642000 & 6329000 & \\
\hline & 2.605396000 & 000 & \\
\hline & 77000 & 6000 & \\
\hline & 000 & & \\
\hline & & & \\
\hline & 00 & & \\
\hline & 5000 & 4000 & \\
\hline & 5.072775000 & 000 & \\
\hline & 1.400076000 & 9000 & \\
\hline & 5.357265000 & 1.001607000 & \\
\hline & $6.3 \varepsilon$ & & \\
\hline & & & \\
\hline & 000 & & \\
\hline & & & \\
\hline & 000 & & \\
\hline & 00 & & \\
\hline & 000 & 00 & \\
\hline & 1000 & 000 & \\
\hline & 0.29 & -2.5 & \\
\hline & 0.07 & & \\
\hline & 05 & & \\
\hline & & 00 & \\
\hline & -1.77 & 000 & \\
\hline & & & \\
\hline & -4.1 & & \\
\hline & -3.9 & & \\
\hline & -5.5 & -0 & \\
\hline & -6.3 & -1. & \\
\hline & -5.75 & & \\
\hline & $-6.7 \varepsilon$ & & \\
\hline & -4.7 & & \\
\hline & -4.8 & & \\
\hline & & & \\
\hline & 00 & -1. & \\
\hline & -0.9 & & \\
\hline & & & \\
\hline & -0. & & \\
\hline & & & \\
\hline & 000 & 1.0 & \\
\hline & -0.66 & & \\
\hline & & & \\
\hline & & & \\
\hline & & & \\
\hline & & & \\
\hline & & & \\
\hline & & & \\
\hline & -0.1 & 00 & \\
\hline & & 00 & \\
\hline & 2. & -0.35 & 2.1 \\
\hline
\end{tabular}




\begin{tabular}{|c|c|c|c|}
\hline & & & \\
\hline & ח & & \\
\hline & .234009000 & .571813000 & \\
\hline & 2.022543000 & .482811000 & 5.702790000 \\
\hline & 1.969431000 & -0.220112000 & 6000 \\
\hline & 0.823493000 & 5000 & 7000 \\
\hline & 79000 & 533000 & -2 \\
\hline & 3000 & 000 & \\
\hline & & & \\
\hline & 00 & 000 & \\
\hline & 000 & 000 & \\
\hline & 4000 & -0.6 & -2.4 \\
\hline & -0.942973000 & 2.384442000 & -1.7 \\
\hline & 0.17 & 000 & -3.1 \\
\hline & -1.1 & 1.6 & \\
\hline & & -0.5 & -3 \\
\hline & 000 & 000 & -4 \\
\hline & 3.19 & 00 & \\
\hline & 1.62 & 00 & \\
\hline & 3.4 & & \\
\hline & 0.62 & & \\
\hline & 4.16 & 00 & -5.2 \\
\hline & 000 & & \\
\hline & 5.15 & & \\
\hline & & & \\
\hline & 3.86 & & \\
\hline & 4.62 & 00 & -6. \\
\hline & 6000 & 000 & 0.1 \\
\hline & -3.66 & 00 & -0. \\
\hline & -2.3 & & \\
\hline & -3.7 & & -1 \\
\hline & -1.3 & 00 & \\
\hline & -4.7 & & 0.0 \\
\hline & -3.45 & 3.66 & \\
\hline & -5.76 & 2.66 & -0. \\
\hline & -3.35 & 4.2 & \\
\hline & -4.6 & 00 & 800 \\
\hline & & 000 & 00 \\
\hline & -0.2 & & -2 \\
\hline & & & \\
\hline & -1.5 & -2.8 & -2.3 \\
\hline & & $-2.4 \mathrm{c}-\mathrm{x}-\mathrm{s}$ & -2.6 \\
\hline & 6000 & -2.205014000 & -2.2 \\
\hline & 5000 & 5000 & -2.5 \\
\hline & -1.61 & -4.212105000 & -2.3 \\
\hline & & 6000 & -2 \\
\hline & & -4.6 & \\
\hline & & & \\
\hline & & & \\
\hline
\end{tabular}

\section{${ }^{1} 5 \mathrm{VI}$-ABBB}

$\begin{array}{lrrr}\text { Fe } & 0.184251000 & 0.166260000 & 0.321693000 \\ \text { O } & -2.399236000 & 0.650760000 & 2.665431000 \\ \text { C } & 2.607826000 & -1.399377000 & -0.244865000 \\ \text { H } & 3.162652000 & -2.353167000 & -0.236695000 \\ \text { O } & 1.491077000 & 1.326006000 & -0.338440000 \\ \text { C } & 3.262603000 & -0.275257000 & -0.848302000 \\ \text { N } & -0.763094000 & -0.969072000 & 1.669013000 \\ \text { C } & 4.569512000 & -0.450421000 & -1.396863000 \\ \text { H } & 4.985495000 & -1.466110000 & -1.438131000 \\ \text { N } & 1.433420000 & -1.352427000 & 0.329031000 \\ \text { C } & 5.314929000 & 0.632809000 & -1.851519000 \\ \text { H } & 6.314604000 & 0.481011000 & -2.274714000\end{array}$


$\begin{array}{llll}\text { C } & 4.768670000 & 1.941568000 & -1.755224000\end{array}$

$\begin{array}{llll}\text { H } & 5.349312000 & 2.800461000 & -2.114607000\end{array}$

$\begin{array}{llll}\text { C } & 3.494206000 & 2.145888000 & -1.236558000\end{array}$

H $\quad 3.050276000 \quad 3.146592000 \quad-1.182690000$

$\begin{array}{llll}\text { C } & 2.692747000 & 1.049375000 & -0.793801000\end{array}$

$\begin{array}{llll}\text { C } & 0.914928000 & -2.589888000 & 0.942959000\end{array}$

H $\quad 1.727028000 \quad-3.248394000 \quad 1.298483000$

H $\quad 0.349215000 \quad-3.148292000 \quad 0.172106000$

$\begin{array}{llll}\text { C } & 0.022125000 & -2.154346000 & 2.093005000\end{array}$

$\begin{array}{llll}\text { H } & -0.671195000 & -2.952985000 & 2.412062000\end{array}$

$\begin{array}{llll}\text { H } & 0.619057000 & -1.840467000 & 2.962828000\end{array}$

$\begin{array}{llll}\text { C } & -1.576780000 & -1.069815000 & 0.556060000\end{array}$

$\begin{array}{llll}\text { C } & -2.892450000 & -0.446470000 & 0.558704000\end{array}$

C $\quad-3.803076000 \quad-0.619713000 \quad-0.509022000$

H $\quad-3.520953000 \quad-1.265618000 \quad-1.349388000$

$\begin{array}{llll}\text { C } & -5.043437000 & 0.030011000 & -0.513678000\end{array}$

H $\quad-5.734937000 \quad-0.111394000 \quad-1.352816000$

$\begin{array}{llll}\text { C } & -5.392866000 & 0.869114000 & 0.565408000\end{array}$

$\begin{array}{llll}\text { H } & -6.359069000 & 1.389004000 & 0.566978000\end{array}$

$\begin{array}{llll}\text { C } & -4.506831000 & 1.056709000 & 1.636178000\end{array}$

$\begin{array}{llll}\text { H } & -4.755430000 & 1.719095000 & 2.474100000\end{array}$

$\begin{array}{llll}\text { C } & -3.250955000 & 0.416878000 & 1.634536000\end{array}$

H $\quad-1.447128000 \quad-1.968069000 \quad-0.059677000$

$\begin{array}{llll}\text { B } & -0.985776000 & 0.249648000 & 2.652605000\end{array}$

$\begin{array}{llll}\text { O } & -0.084306000 & 1.281992000 & 1.997829000\end{array}$

$\begin{array}{llll}\text { O } & -0.481799000 & 0.074813000 & 3.981435000\end{array}$

C $\quad 0.999934000 \quad 1.612032000 \quad 2.953741000$

$\begin{array}{llll}\text { C } & 0.276689000 & 1.276769000 & 4.316010000\end{array}$

$\begin{array}{llll}\text { C } & -0.698723000 & 2.387590000 & 4.744803000\end{array}$

$\begin{array}{llll}\text { C } & 1.224819000 & 0.936389000 & 5.466576000\end{array}$

$\begin{array}{llll}\text { C } & 1.347632000 & 3.081261000 & 2.725297000\end{array}$

$\begin{array}{llll}\text { C } & 2.239953000 & 0.737613000 & 2.743275000\end{array}$

$\begin{array}{llll}\text { H } & 1.824925000 & 3.184968000 & 1.735282000\end{array}$

$\begin{array}{llll}H & 0.445762000 & 3.713352000 & 2.741570000\end{array}$

$\begin{array}{llll}\text { H } & 2.627782000 & 0.869539000 & 1.724230000\end{array}$

$\begin{array}{llll}H & -0.154487000 & 3.280523000 & 5.099111000\end{array}$

$\begin{array}{llll}H & -1.363237000 & 2.681064000 & 3.915049000\end{array}$

$\begin{array}{llll}H & 2.023008000 & -0.329929000 & 2.897851000\end{array}$

$\begin{array}{llll}\text { H } & 3.031705000 & 1.040678000 & 3.448972000\end{array}$

$\begin{array}{llll}\text { H } & 2.051866000 & 3.438559000 & 3.498013000\end{array}$

$\begin{array}{llll}H & -1.324669000 & 2.012565000 & 5.573222000\end{array}$

$\begin{array}{llll}\text { H } & 1.923052000 & 1.771015000 & 5.656569000\end{array}$

$\begin{array}{llll}H & 1.808158000 & 0.026436000 & 5.252195000\end{array}$

$\begin{array}{llll}\text { H } & 0.640588000 & 0.762352000 & 6.387429000\end{array}$

H $\quad-1.141745000 \quad-0.466123000-2.083288000$

C $\quad 0.687755000 \quad 1.329146000 \quad-3.268515000$

$\begin{array}{llll}\text { C } & 0.741795000 & -0.095945000 & -3.024327000\end{array}$

$\begin{array}{llll}\text { C } & -0.151716000 & 2.246153000 & -2.649561000\end{array}$

H $\quad-1.884719000 \quad 1.663887000 \quad 0.295854000$

C $\quad-0.187533000 \quad-0.903474000 \quad-2.404002000$

$\begin{array}{llll}\text { C } & -1.107375000 & 2.185035000 & -1.548608000\end{array}$

$\begin{array}{llll}\text { H } & -0.087271000 & 3.260776000 & -3.072592000\end{array}$

$\begin{array}{llll}\text { C } & -1.055635000 & 1.438026000 & -0.388564000\end{array}$

H $\quad 1.595515000 \quad-0.608919000 \quad-3.486690000$

C $\quad 1.622082000 \quad 1.840462000 \quad-4.325569000$

C $\quad 2.936947000 \quad 1.327637000 \quad-4.453926000$

$\begin{array}{llll}\text { C } & 1.221985000 & 2.848109000 & -5.239960000\end{array}$

$\begin{array}{llll}\text { H } & 3.293751000 & 0.561322000 & -3.756284000\end{array}$

H $\quad 0.198249000 \quad 3.241825000 \quad-5.197391000$

C $\quad 3.820292000 \quad 1.816682000 \quad-5.429511000$

$\begin{array}{llll}\text { C } & 2.102378000 & 3.337311000 & -6.219468000\end{array}$

H $\quad 4.838906000 \quad 1.411227000 \quad-5.484450000$

H $\quad 1.759205000 \quad 4.113377000 \quad-6.915983000$

$\begin{array}{llll}\text { C } & 3.410713000 & 2.827182000 & -6.318970000\end{array}$

$\begin{array}{llll}\text { H } & 4.099467000 & 3.208729000 & -7.083345000\end{array}$

$\begin{array}{lrrr}\text { C } & -2.241288000 & 3.167326000 & -1.670848000\end{array}$ 


$\begin{array}{lrrr}\text { C } & -2.824296000 & 3.429347000 & -2.936697000 \\ \text { C } & -2.784127000 & 3.842599000 & -0.547985000 \\ \text { H } & -2.435725000 & 2.909470000 & -3.822848000 \\ \text { H } & -2.339119000 & 3.681857000 & 0.442240000 \\ \text { C } & -3.907566000 & 4.314968000 & -3.074449000 \\ \text { C } & -3.870406000 & 4.720929000 & -0.680917000 \\ \text { H } & -4.342204000 & 4.490856000 & -4.067419000 \\ \text { H } & -4.267360000 & 5.229252000 & 0.207641000 \\ \text { C } & -4.440279000 & 4.964085000 & -1.945784000 \\ \text { H } & -5.287340000 & 5.654257000 & -2.050038000 \\ \text { C } & -0.071492000 & -2.366246000 & -2.360522000 \\ \text { C } & 1.174491000 & -3.037702000 & -2.496895000 \\ \text { C } & -1.224446000 & -3.168912000 & -2.146403000 \\ \text { H } & 2.088541000 & -2.456739000 & -2.667180000 \\ \text { H } & -2.208092000 & -2.687721000 & -2.082094000 \\ \text { C } & 1.264910000 & -4.431864000 & -2.384928000 \\ \text { C } & -1.135928000 & -4.566160000 & -2.035318000 \\ \text { H } & 2.244459000 & -4.918328000 & -2.475646000 \\ \text { H } & -2.045990000 & -5.154795000 & -1.863938000 \\ \text { C } & 0.112452000 & -5.206403000 & -2.141836000 \\ \text { H } & 0.187994000 & -6.296217000 & -2.041889000\end{array}$

\section{${ }^{1}$ 5VII FSPE: -3494.313934278949 $E_{\mathrm{h}}$}

\begin{tabular}{|c|c|c|c|}
\hline & 00 & 0 & \\
\hline & 00 & 000 & \\
\hline & 000 & 000 & \\
\hline & 000 & 000 & \\
\hline & 0.625509000 & 1.173894000 & \\
\hline & 2.599619000 & -0.087830000 & \\
\hline & 000 & 00 & \\
\hline & 000 & -0.1 & \\
\hline & 00 & $-1 . C$ & \\
\hline & 4000 & -1.1 & \\
\hline & 5000 & 6000 & -3.7 \\
\hline & 93000 & 0.731013000 & -4.4 \\
\hline & 72000 & 1.911073 & -3.8 \\
\hline & 3000 & 2.681935000 & -4.6 \\
\hline & 4000 & 2.019261000 & -3.0 \\
\hline & 6000 & 00 & -3. \\
\hline & 0 & 0 & \\
\hline & 1.40 & -2.2 & \\
\hline & 2.41 & -2.6 & \\
\hline & 0.80 & -3.0 & 0.55 \\
\hline & 3000 & -1.6 & 2.2 \\
\hline & 2000 & -2.4 & 2.9 \\
\hline & 00 & -1.0 & $2 . \varepsilon$ \\
\hline & 0 & -1.2 & 1.08 \\
\hline & -2.7 & -0.6 & 1.1 \\
\hline & -3.7 & & \\
\hline & 4000 & -1.7 & \\
\hline & 3000 & 50000 & 0.4 \\
\hline & -5.8 & 198000 & -0.2 \\
\hline & 0000 & 0.5 & 1.4 \\
\hline & 9000 & 000 & 1.56 \\
\hline & -4.27 & & 300 \\
\hline & -4.4 & & 3.1 \\
\hline & 3000 & & 2.22 \\
\hline & 61000 & -2.267164000 & \\
\hline 3 & -0.582563000 & 0.639412000 & \\
\hline O & 12000 & 1.720772000 & 1.7627 \\
\hline 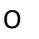 & 0.278281000 & 0.749871000 & 3.84852800 \\
\hline C & 0.951 & 2.410998000 & \\
\hline & 0.813928000 & 2.109161000 & 3.84045000 \\
\hline
\end{tabular}




\begin{tabular}{|c|c|c|c|}
\hline & 2000 & 75000 & 0000 \\
\hline & 2.135888000 & 2.109424000 & 4.608618000 \\
\hline & 0.850128000 & 3.886192000 & 1.918934000 \\
\hline & 2.224592000 & 1.803979000 & 1.691169000 \\
\hline & 0.912793000 & 3.982854000 & 0.821525000 \\
\hline & -0.109919000 & 4.319008000 & 2.234209000 \\
\hline & 2.161587000 & 1.874234000 & 0.592879000 \\
\hline & 0.191412000 & 4.063976000 & 4.629704000 \\
\hline & -1.142745000 & 3.086475000 & 3.939370000 \\
\hline & 2.330880000 & 0.739470000 & 1.957716000 \\
\hline H & 3.128161000 & 2.343356000 & 2.024547000 \\
\hline & 1.675741000 & 4.463504000 & 2.373702000 \\
\hline & -0.434523000 & 2.646923000 & 5.527066000 \\
\hline & 2.639508000 & 3.088286000 & 4.513166000 \\
\hline & 2.815070000 & 1.324475000 & 4.237104000 \\
\hline & 1.948435000 & 1.924219000 & 5.681483000 \\
\hline & -0.529361000 & -0.904751000 & -1.039820000 \\
\hline e & -2.429606000 & 2.101156000 & -0.888599000 \\
\hline$C$ & -2.060524000 & 3.458266000 & -1.043037000 \\
\hline C & -2.343370000 & 1.209094000 & -1.960197000 \\
\hline H & -2.628616000 & 0.162962000 & -1.803249000 \\
\hline c & -1.645218000 & 3.863384000 & -2.331065000 \\
\hline 0 & -1.888734000 & 1.608234000 & -3.235496000 \\
\hline $\mathrm{H}$ & -1.358594000 & 4.907475000 & -2.502189000 \\
\hline C & -1.553404000 & 2.978440000 & -3.430490000 \\
\hline H & -2.763082000 & 1.718660000 & 0.079548000 \\
\hline C & -1.760863000 & 0.571232000 & -4.296164000 \\
\hline C & -2.409267000 & 0.689909000 & -5.546876000 \\
\hline C & -0.960716000 & -0.568679000 & -4.048195000 \\
\hline H & -3.028149000 & 1.573098000 & -5.748780000 \\
\hline H & -0.444702000 & -0.651475000 & -3.081383000 \\
\hline c & -2.257776000 & -0.304596000 & -6.526511000 \\
\hline c & -0.803856000 & -1.560888000 & -5.031127000 \\
\hline $\mathrm{H}$ & -2.769631000 & -0.200216000 & -7.491855000 \\
\hline $\mathrm{H}$ & -0.169118000 & -2.433064000 & -4.827939000 \\
\hline c & -1.451295000 & -1.431534000 & -6.274134000 \\
\hline $\mathrm{H}$ & -1.328536000 & -2.204499000 & -7.043606000 \\
\hline C & -1.091703000 & 3.523991000 & -4.742729000 \\
\hline c & -1.614763000 & 4.74 & -5.221506000 \\
\hline C & -0.113730000 & 2.865 & -5.527741000 \\
\hline $\mathrm{H}$ & -2.389359000 & 5.267543000 & -4.640934000 \\
\hline $\mathrm{H}$ & 0.310009000 & 1.918314000 & -5.178671000 \\
\hline C & -1.175837000 & 5.299961000 & -6.438394000 \\
\hline c & 0.331412000 & 3.417436000 & -6.738818000 \\
\hline $\mathrm{H}$ & -1.603065000 & 6.247253000 & -6.791804000 \\
\hline $\mathrm{H}$ & 1.098299000 & 2.891945000 & -7.322176000 \\
\hline c & -0.198041000 & 4.636818000 & -7.202228000 \\
\hline $\mathrm{H}$ & 0.147355000 & 5.065546000 & -8.151610000 \\
\hline c & -2.110760000 & 4.407414000 & 0.104095000 \\
\hline C & -2.770790000 & 4.060583000 & 1.309733000 \\
\hline C & -1.491793000 & 5.681270000 & 0.044547000 \\
\hline $\mathrm{H}$ & -3.258503000 & 3.085589000 & 1.408081000 \\
\hline $\mathrm{H}$ & -0.942586000 & 5.984717000 & -0.854310000 \\
\hline c & -2.811896000 & 4.940716000 & 2.401255000 \\
\hline c & -1.531206000 & 6.565075000 & 1.134863000 \\
\hline $\mathrm{H}$ & -3.328140000 & 4.636363000 & 3.320523000 \\
\hline r & -1.032514000 & 7.539768000 & 1.058404000 \\
\hline C & -2.191887000 & 6.201346000 & 2.322772000 \\
\hline & -2.218455000 & 6.888737000 & 3.177528000 \\
\hline
\end{tabular}




\section{References}

[1] Gallagher, K. J.; Webster, R. L. Chem. Commun. 2014, 50, 12109-12111.

[2] Chapman, M. R.; Henkelis, S. E.; Kapur, N.; Nguyen, B. N.; Willans, C. E. ChemistryOpen 2016, 5, 351-356.

[3] Kumar, S.; Vaidya, S.; Pissas, M.; Sanakis, Y.; Gupta, R. Eur. J. Inorg. Chem. 2012, 55255533.

[4] Davies, J. E.; Gatehouse, B. M. Acta Cryst. 1973, B29, 1934-1942.

[5] Coggon, B. P.; Mcphail, A. T.; McLachlan, V. N.; Mabbs, F. E. Inorg. Phys. Theor. 1971, 1014-1019.

[6] Koner, S.; lijima, S.; Watanabe, M.; Sato, M. J. Coord. Chem. 2003, 56, 103-111.

[7] Eguchi, H.; Umemura, M.; Kurotani, R.; Fukumura, H.; Sato, I.; Kim, J. H.; Hoshino, Y.; Lee, J.; Amemiya, N.; Sato, M.; Hirata, K.; Singh, D. J.; Masuda, T.; Yamamoto, M.; Urano, T.; Yoshida, K.; Tanigaki, K.; Yamamoto, M.; Sato, M.; Inoue, S.; Aoki, I.; Ishikawa, Y. Sci. Rep. 2015, 5, 1-14.

[8] McKenzie, E. D.; Towl, A. D. C.; Gerloch, M. Inorg. Phys. Theor. 1969, 2850-2858.

[9] Oyaizu, K.; Dewi, E. L.; Tsuchida, E. Inorganica Chim. Acta 2001, 321, 205-208.

[10] Ashmawy, F. M.; Ujaimi, A. R.; Parish, R. V.; Pritchard, R. G.; McAuliffe, C. A. Inorganica Chim. Acta 1991, 187, 155-158.

[11] Yan, J. H.; Shen, X. P.; Zhou, H. Acta Crystallogr. Sect. E Struct. Reports Online 2010, 66, 112-122.

[12] Tanaka, K.; Toyoda, K.; Wada, A.; Shirasaka, K.; Hirano, M. Chem. Eur. J. 2005, 11, 11451156.

[13] Neese, F. WIREs Comput. Mol. Sci. 2012, 2, 73-78.

[14] Becke, A. D. Phys. Rev. A 1988, 38, 3098-3100.

[15] Perdew, J. P. Phys. Rev. B 1986, 33, 8822-8824.

[16] van Lenthe, E.; Baerends, E. J.; Snijders, J. G. J. Chem. Phys. 1994, 101, 9783-9792.

[17] van Lenthe, E.; Snijders, J. G.; Baerends, E. J. J. Chem. Phys. 1996, 105, 6505-6516.

[18] van Wüllen, C. J. Chem. Phys. 1998, 109, 392-399.

[19] Schäfer, A.; Huber, C.; Ahlrichs, R. J. Chem. Phys. 1994, 100, 5829-5835.

[20] Weigend, F. Phys. Chem. Chem. Phys. 2006, 8, 1057-1065.

[21] Pantazis, D. A.; Chen, X. Y.; Landis, C. R.; Neese, F. J. Chem. Theory. Comput. 2008, 4, 908-919.

[22] Pantazis, D. A.; Neese, F. WIREs Comput. Mol. Sci. 2014, 4, 363-374.

[23] Grimme, S.; Antony, J.; Ehrlich, S.; Krieg, H. J. Chem. Phys. 2010, 132, 154104-154119.

[24] Barone, V.; Cossi, M. J. Phys. Chem. A 1998, 102, 1995-2001. 